\title{
[poj p'endezel'e maqal'žin]
}

Anja Behnke

Syntaktische Strukturen im Selkupischen

Eine korpusbasierte Untersuchung der zentralen und südlichen Dialekte

[na tängədəl puale qwərnn] 



\section{Syntaktische Strukturen im Selkupischen}

Eine korpusbasierte Untersuchung der zentralen und südlichen Dialekte

Anja Behnke

Logos Verlag Berlin 
Bibliografische Information der Deutschen Nationalbibliothek

Die Deutsche Nationalbibliothek verzeichnet diese Publikation in der Deutschen Nationalbibliografie; detaillierte bibliografische Daten sind im Internet über http://dnb.d-nb.de abrufbar.

\section{(c) $(1) \ominus$}

(C) Copyright Logos Verlag Berlin GmbH 2021

Alle Rechte vorbehalten.

ISBN 978-3-8325-5182-7

Logos Verlag Berlin GmbH

Georg-Knorr-Str. 4, Geb. 10, 12681 Berlin

Tel.: $\quad+49(0) 30 / 42851090$

Fax: $\quad+49(0) 30 / 42851092$

http://www.logos-verlag.de 
Für Dirk

Wegbegleiter und enger Freund 



\section{Danksagung}

Der Weg der vorliegenden Arbeit von der ersten Idee bis zur Fertigstellung war durchaus nicht linear, sondern von vielen Höhen und Tiefen gekennzeichnet. Die Begeisterung für wenig erforschte, vom Aussterben bedrohte Sprachen sowie eine gewisse mir eigene Naivität bei der Herangehensweise an neue Projekte waren stetig Motivation und zeitweilig Ursache für Frustration gleichermaßen. Herausfordernd war insbesondere zu Beginn das noch unvollständige elektronische Textkorpus, was zur Folge hatte, dass viele syntaktische Phänomene händisch gesucht werden mussten. Eine große Anzahl von Menschen war an der Arbeit direkt oder indirekt beteiligt. Bei allen bedanke ich mich auf diesem Wege, auch wenn sie hier nicht namentlich genannt werden. Allen voran möchte ich mich bei meiner Betreuerin Prof. Dr. Beáta Wagner-Nagy bedanken, die mir beständig bei Fragen zur Verfügung stand und die Arbeit mit konstruktiver Kritik vorangebracht hat. Meiner Zweitgutachterin Prof. Dr. Susann Fischer danke ich für konstruktive Gespräche aus einer anderen Perspektive. Ich danke meinen Kollegen Josefina Budzisch, Dr. Chris Lasse Däbritz und Hannah Wegener nicht nur für den regelmäßigen und beständigen Austausch zu fachlichen Fragen und Problemen, sondern auch die Möglichkeit, Frustrationen und Blockaden in die Welt lassen zu können.

Meiner Lektorin und Freundin Susanne Bienwald danke ich für das überaus schnelle Lektorat der Arbeit.

Und schließlich danke ich meiner Familie, allen voran meiner Tochter Lilith, die einige Jahre damit groß geworden ist, das "Mama ein Buch schreibt" und besonders in der Endphase ihren Freunden, die sie besuchen kommen wollten, sagen musste: „Das geht erst, wenn Mamas Buch fertig ist“. Meine Mutter Marina Langenbach hat mir oft den Rücken freigehalten, indem sie meine Tochter betreut hat; dafür danke ich ihr sehr. Und mein Partner Jörn Hansen musste sicher häufiger Gespräche über selkupische Syntax aushalten, als ihm lieb war. Auch ihm danke ich für seine Geduld und Aufmunterung in schwierigen Zeiten. 



\section{Inhaltsverzeichnis}

Abbildungsverzeichnis $\quad$ v

Tabellenverzeichnis vii

1 Einführung 1

1.1 Theoretische Orientierung . . . . . . . . . . . . 3

1.2 Das Selkupische. . . . . . . . . . . . . . . . . . . 4

1.3 Sprachdaten . . . . . . . . . . . . . . . 6

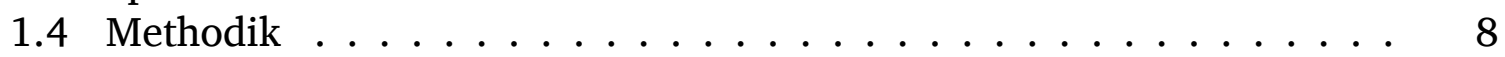

1.5 Forschungsstand ... . . . . . . . . . . . . . 14

1.6 Typologischer Abriss . . . . . . . . . . . . . . . . . . . . . 15

2 Phrasentypen 19

2.1 Nominalphrase ..... . . . . . . . . . . . . . . . . . 19

2.1 .1 Nominale Köpfe . . . . . . . . . . . . . . . . . . . 19

2.1 .2 Modifikatoren .... . . . . . . . . . . . . . . . 21

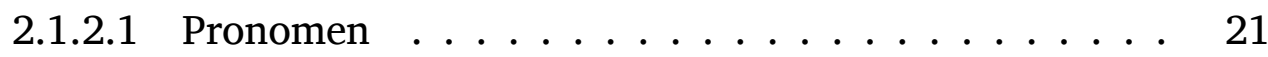

2.1 .2 .2 Possessivpronomen . . . . . . . . . . . . . . . 22

2.1 .2 .3 Interrogativpronomen . . . . . . . . . . . . . . 28

2.1 .2 .4 Indefinitpronomen. ... . . . . . . . . . . . . . 29

2.1 .2 .5 Demonstrativpronomen . . . . . . . . . . . . 31

2.1.2.6 Emphatisches Pronomen . . . . . . . . . . . . . 33

2.1 .2 .7 Adjektive . . . . . . . . . . . . . . . 34

2.1 .2 .8 Numeralia . . . . . . . . . . . . . . . . . . . . . . . . . 35

2.1 .2 .9 Quantifizierer . . . . . . . . . . . . . . . . 37

2.1.2.10 Partizipien ................. . . . 39

2.1.2.11 Nominalphrase als Modifikator . . . . . . . . . . . 41

2.1.2.12 Multiple Modifikatoren . . . . . . . . . . . . 44

2.1.2.13 Elliptische Phrasen . . . . . . . . . . . . . . . . . 44

2.2 Negierte Nominal phrasen ... . . . . . . . . . . . . . 45

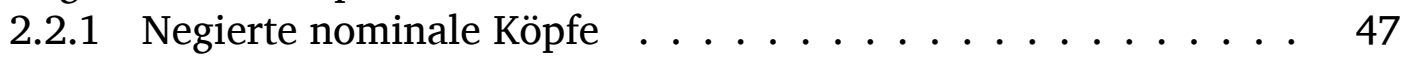

2.2 .2 Negierte Modifikatoren . . . . . . . . . . . . . . . 50

2.2.2.1 Negierte Possessivpronomen . . . . . . . . . . . . 51

2.2 .2 .2 Negierte Interrogativpronomen . . . . . . . . . . . 51

2.2 .2 .3 Negierte Adjektive . . . . . . . . . . . . . 52 


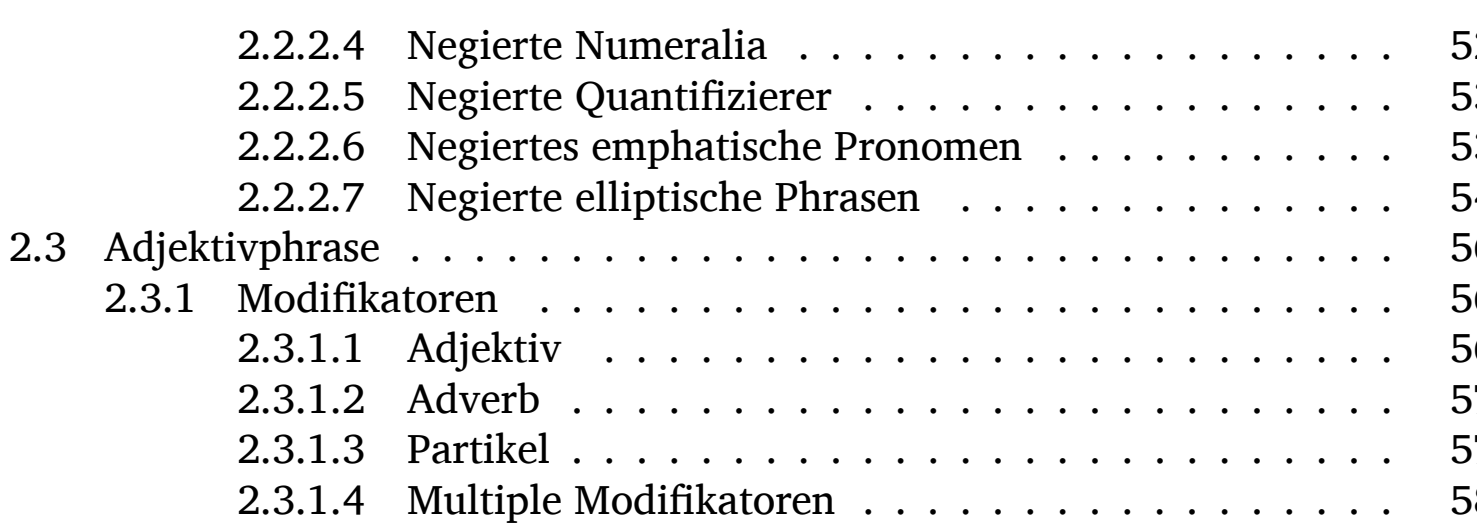

2.4 Negierte Adjektivphrase . . . . . . . . . . . . . . . . . 59

2.5 Adverbphrase ........ . . . . . . . . . . . . . . . 60

2.5 .1 Modifikatoren . . . . . . . . . . . . . . . 60

2.5.1.1 Partikel . . . . . . . . . . . . . . . 60

2.6 Negierte Adverbphrase . . . . . . . . . . . . . . . . . . 61

2.7 Adpositionalphrase...... . . . . . . . . . . . . . 62

2.7 .1 Einfache Postpositionen . . . . . . . . . . . . . 62

2.7 .2 Komplexe Postpositionen . . . . . . . . . . . . . . . . 68

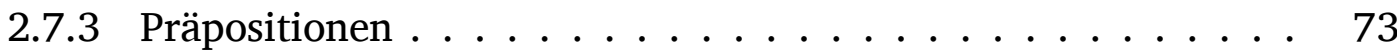

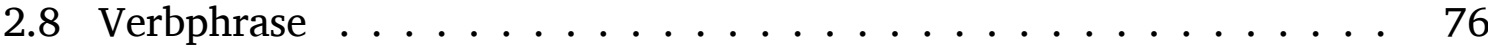

2.8 .1 Modifikatoren . . . . . . . . . . . . . 78

2.8 .1 .1 Präverb . . . . . . . . . . . . . . . . . 79

2.8 .1 .2 Adverb ...... . . . . . . . . . . 79

2.8 .1 .3 Infinite Verbformen . . . . . . . . . . . . 80

2.9 Negierte Verbphrase . . . . . . . . . . . . . . . . . . 84

3 Syntax von Sätzen 89

3.1 Satzglieder und grammatische Relationen . . . . . . . . . . . . . 89

3.1 .1 Subjekt ........................ 90

3.1 .2 Direktes Objekt. . . . . . . . . . . . . . . . . . . 92

3.1 .3 Indirektes Objekt . . . . . . . . . . . . . . . . . . . 96

3.1 .4 Adjunkte $\ldots \ldots \ldots \ldots \ldots \ldots \ldots$

3.2 Wortstellung . . . . . . . . . . . . . . . 97

4 Einfacher Satz $\quad 99$

4.1 Deklarativsatz . . . . . . . . . . . . . . . . . . . . 99

4.1 .1 Intransitiver Deklarativsatz . . . . . . . . . . . . . . . 99

4.1 .2 Transitiver Deklarativsatz . . . . . . . . . . . . . . . . . 100

4.1 .2 .1 Monotransitiver Satz . . . . . . . . . . . 100

4.1 .2 .2 Ditransitiver Satz . . . . . . . . . . . . . . 102

4.1 .3 Kopulasätze... . . . . . . . . . . . . . . . . . . 110

4.1 .4 Verblose Sätze ......... . . . . . . . . . . . 116

4.1 .5 Unpersönliche Konstruktionen . . . . . . . . . . . . . . 120

4.2 Interrogativsätze $\ldots \ldots \ldots \ldots \ldots \ldots \ldots \ldots \ldots \ldots \ldots$ 
4.2 .1 w-Interrogativsätze . . . . . . . . . . . . . . . . 126

4.2 .2 Polare Interrogativsätze . . . . . . . . . . . . . 130

4.3 Imperativsätze . . . . . . . . . . . . . . . . . . . . . . . 131

4.4 Negation einfacher Sätze ... . . . . . . . . . . . . . . . . . . 133

4.4 .1 Negierter Deklarativsatz . . . . . . . . . . . . . . . . 133

4.4.1.1 Negierter intransitiver Deklarativsatz . . . . . . . . 134

4.4.1.2 Negierter monotransitiver Deklarativsatz . . . . . . . 134

4.4 .1 .3 Negierter ditransitiver Satz . . . . . . . . . . . . . . 135

4.4 .2 Negierte Kopulasätze. . . . . . . . . . . . . . . . . . . 137

4.4 .3 Negierte verblose Sätze . . . . . . . . . . . . . . . . . . . . 142

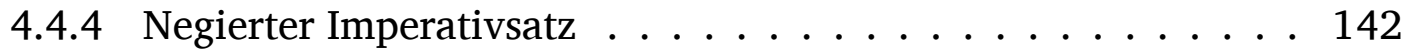

4.4 .5 Negierter Interrogativsatz . . . . . . . . . . . . . . . . . . . 144

5 Komplexer Satz 147

5.1 Koordination . . . . . . . . . . . . . . . . . . . . . 148

5.1 .1 Juxtaposition . . . . . . . . . . . . . . . . . . . . . 148

5.1 .2 Infinite Verbformen . . . . . . . . . . . . . . . . . 149

5.1 .3 Konjunktionalsätze . . . . . . . . . . . . . . . . . . . . 151

5.1 .3 .1 Additiv . . . . . . . . . . . . . 151

5.1 .3 .2 Adversativ . . . . . . . . . . . . . . 154

5.1 .3 .3 Disjunktiv . . . . . . . . . . . . . . . 154

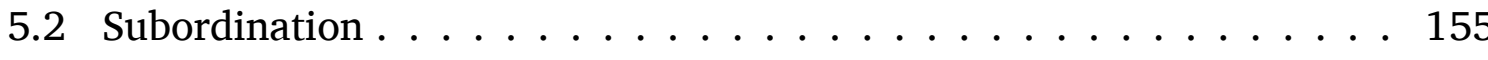

5.2 .1 Juxtaposition . . . . . . . . . . . . . . . . . . 155

5.2 .2 Infinite Verbalformen . . . . . . . . . . . . . . 157

5.2 .2 .1 Subordinierende Konverbsätze . . . . . . . . . . . 157

5.2 .2 .2 Subordinierter Infinitivsatz . . . . . . . . . . . . . 158

5.2 .3 Konjunktionalsätze. . . . . . . . . . . . . . . . . . . . . . . . 159

5.2 .3 .1 Komplementsätze . . . . . . . . . . . . . . . . . . 160

5.2 .3 .2 Adverbialsätze . . . . . . . . . . . . . . . . 162

5.2 .3 .3 Finalsatz $\ldots \ldots \ldots$. . . . . . . . . . . . . . 162

5.2 .3 .4 Kausalsatz ... . . . . . . . . . . . . . . . 163

5.2 .3 .5 Komparativsatz . . . . . . . . . . . . . . . . 164

5.2 .3 .6 Konditionalsatz . . . . . . . . . . . . . . . . 166

5.2 .3 .7 Konsekutivsatz . . . . . . . . . . . . . . . 167

5.2 .3 .8 Konzessivsatz. . . . . . . . . . . . . . . . . . . 167

5.2 .3 .9 Temporalsatz . . . . . . . . . . . . . . . . . 168

5.2 .4 Relativsätze. . . . . . . . . . . . . . . . . 168

5.2.4.1 Kombination von Koordination und Subordination . . 170

5.3 Negation komplexer Sätze . . . . . . . . . . . . . . . . . 170

5.3 .1 Koordination negierter Sätze ... . . . . . . . . . . . 171

5.3 .1 .1 Negation bei Juxtaposition . . . . . . . . . . . . . . 171

5.3 .1 .2 Negierte koordinierte Konverbsätze _... . . . . . . . 173

5.3.1.3 Negierte koordinierte Konjunktionalsätze . . . . . . . 173

5.3.2 Subordination negierter Sätze . . . . . . . . . . . . . 174 
5.3.2.1 Negierte subordinierte Konverbsätze . . . . . . . . . . 174

5.3 .2 .2 Subordinierter Infinitivsatz . . . . . . . . . . . . 175

5.3 .2 .3 Negierter Komplementsatz . . . . . . . . . . . . 175

5.3 .2 .4 Negierter Adverbialsatz . . . . . . . . . . . . . 177

5.3 .2 .5 Negierter Relativsatz $\ldots \ldots \ldots \ldots$. . . . . . . 181

6 Exkurs Konverbkonstruktionen $\quad 185$

6.1 Einleitung . . . . . . . . . . . . . . . . . . 185

6.2 Konverbverbindungen in uralischen Sprachen . . . . . . . . . . . 187

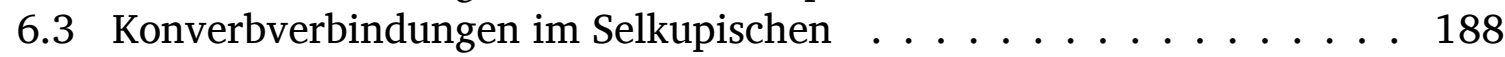

6.4 Komplexe (konverbale) Prädikate . . . . . . . . . . . . . . . . . . . . . 189

6.4 .1 Gruppe A . . . . . . . . . . . . . . . . . . . . 189

6.4 .2 Gruppe B . . . . . . . . . . . . . . . . . . 193

6.4 .3 Gruppe C . . . . . . . . . . . . . . . . . . 195

6.4 .4 Gruppe D ... . . . . . . . . . . . . . . . 196

6.4.4.1 Initialtransformative Auxiliare . . . . . . . . . . 198

6.4 .4 .2 Finaltransformative Auxiliare. . . . . . . . . . 200

6.4 .4 .3 Nontransformative Auxiliare . . . . . . . . . . 202

6.5 Zusammenfassung $\ldots \ldots \ldots \ldots \ldots \ldots \ldots \ldots$

7 Zusammenfassung und Ausblick 209

$\begin{array}{ll}\text { A Graphemübersicht } & 215\end{array}$

\begin{tabular}{ll}
\hline B Textsammlungen & 217
\end{tabular}

\begin{tabular}{ll}
\hline C Abkürzungen und Symbole & 221
\end{tabular}

\begin{tabular}{ll}
\hline Literaturverzeichnis & 223
\end{tabular}

$\begin{array}{ll}\text { Stichwortverzeichnis } & 231\end{array}$ 


\section{Abbildungsverzeichnis}

Abb. 1: Siedlungsgebiete der Selkupen ................ 5

Abb. 2: $\quad$ Textbeispiel im Partitur-Editor inkl. Transkriptions- und 9

Abb. 3: Korpussuche in EXAKT: Annotationssuche mittels einfacher Zeichenketten, Suchergebnis als KWiC-Konkordanz dargestellt. ...... 11

Abb. 4: Korpussuche in EXAKT: Suchausdruck mittels regulärer Ausdrücke, Suchergebnis als KWIC-Konkordanz dargestellt. ........ 13

Abb. 5: Grammatikalisierungsprozess in konverbalen Konstruktionen . . . . 205 



\section{Tabellenverzeichnis}

\begin{tabular}{|c|c|c|}
\hline Tab. 2: & aterialbasis & \\
\hline Tab. 3: & Possessivpronomen 1. Person und px-Markierung des Kopfnomens & \\
\hline ab. 4: & Possessivpronomen 2. Person und PX-Markierung des Kopfnomens & \\
\hline Tab. 5: & Possessivpronomen 3 , Person & \\
\hline ab. 6: & Inter & \\
\hline Tab. 7: & Indefinitpronomen & \\
\hline Tab. 8: & Negationswörter im Selkupischen & \\
\hline Tab. 9: & ionen ir & \\
\hline Tab. 10: & alkasus i & \\
\hline Tab. 11: & Relationale Non & \\
\hline Tab. 12: & tionen ir & \\
\hline Tab. 13: & Mus & \\
\hline Tab. 14: & swörte & \\
\hline Tab. 15: & Verteilung von mi - 'geben' & \\
\hline Tab. 16: & Semantische Relationen in & \\
\hline Tab. 17: & he Re & \\
\hline Tab. 18: & nliche K & \\
\hline Tab. 19: & Koordinierende Konjunkti & \\
\hline Tab. 20: & Negationsstrukturen im Se & \\
\hline Tab. 21: & Rea & \\
\hline Tab. 22: & eit von K & \\
\hline Tab. 23: & Kombinationen yon Bewegungsv & \\
\hline \multirow{2}{*}{ Tab. 24: } & Komk & \\
\hline & Zentr & \\
\hline \multirow[t]{2}{*}{ Tab. 25: } & Kombinationen von Bew & \\
\hline & elkupischen & \\
\hline \multirow[t]{2}{*}{ Tab. 26: } & Kombinationen von B & \\
\hline & & \\
\hline b. 27: & Auxiliare in konverb & \\
\hline ab. 28: & Interaktion von Auxiliaren und Aktionalp & \\
\hline b. 29: & erteilung der ein & \\
\hline ab. 30: & Dialektale Verteilung der Auxiliarverbindungen & \\
\hline
\end{tabular}





\section{Einführung}

Das Selkupische (und insbesondere die zentralen und südlichen Dialekte) gehört zu den akut vom Aussterben bedrohten Sprachen. Weil mein Interesse in der analytischen Erforschung sprachlicher Strukturen liegt und gerade im syntaktischen Bereich des Selkupischen viele blinde Flecken liegen, erforsche ich die von Helimski (1998: 549) als einstige „Lingua Franca“ bezeichnete Sprache. Auch das Wissen, die Sprache mit der vorliegenden Arbeit nicht erhalten zu können, hat dieses Interesse nicht gemindert. Indem ich mich eines Bereiches der selkupischen Sprache annehme, der bisher nur wenig dokumentiert ist, möchte ich mit seiner Beschreibung einerseits die Grundlagen für weitere Forschungen legen und andererseits dem strukturellen Flickenteppich, der einem bei der sprachwissenschaftlichen Betrachtung des Selkupischen unwillkürlich in den Sinn kommt, eine anfängliche Form geben. Einen ersten Eindruck des Flickenteppichs geben die Beispiele (1) und (2).

(1) Qaj mat qwal'-l'e qosti-ku k'er'eg'en. what house go.away-CVB know-INF must

'Man muss losgehen und erfahren, was das für ein Haus ist.' (PMP_1961_ForestWoman_flk_366, Middle Ob)

(2) Ade-ľ̌s̆gu nāde e-ha mašik, mat wēšna-k ta-se wait-INT.PF-INF must be-AOR.3SG 1SG.ACC 1sG forever-ADV 2SG-COM el-ni-k, a t'ipēr' praššaj.

live-AOR-3SG.s but now goodbye

'Man musste auf mich warten, ich wollte für immer mit dir leben, aber jetzt Lebewohl.'

(TTD_1964_Frog_flk_77, Tym)

Hier zeigen sich gleich mehrere interessante Phänomene. Da wäre zum einen die unterschiedliche Umsetzung des Konzeptes „müssen“, für die das Selkupische kein originäres Lexem kennt. Im südselkupischen Beispiel (1) wird die aus den Turksprachen stammende Partikel k'er'eg'ey postverbal verwendet, das zentralselkupische Beispiel (2) nutzt ebenfalls eine Partikel (nāde), allerdings ist sie aus dem Russischen entlehnt und tritt präverbal auf. Ein weiteres Phänomen betrifft die Bildung komplexer Sätze: Während im Südselkupischen hierfür ein Konverb (qwal'l'e) verwendet wird, nutzt das zentralselkupische Beispiel neben der originären Juxtaposition (zwischen dem ersten und zweiten Teilsatz) auch die aus dem Russischen entlehnte Konjunktion $a$ - 'aber' (zwischen dem zweiten und dritten Teilsatz). Ebenfalls aus dem Russischen entlehnt sind die Lexeme 'jetzt' (t'ïpēr'), 'immer' (wēšna) 'Lebewohl' 
(praššaj) in Beispiel (2).

Das vorrangige Ziel der vorliegenden Arbeit ist es daher, eine synchrone Beschreibung syntaktischer Strukturen und ihrer Funktionen im Selkupischen, genauer in dessen zentralen und südlichen Dialekten, zu geben. Bei interessanten Phänomenen oder wo es für das Verständnis der gegenwärtigen Strukturen wichtig ist, werden gelegentlich auch diachrone Aspekte berücksichtigt.

Im einführenden Teil ist ein grammatischer Abriss des Selkupischen enthalten. Er soll helfen, häufige Wiederholungen zu vermeiden, indem einige Grundstrukturen vorab dargelegt werden, die in der Beschreibung erscheinen, bevor sie in den entsprechenden Kapiteln ausführlich erläutert werden. Die vorliegende Arbeit ist formbasiert (analytisch): Die Beschreibung einer Einheit geht stets von ihrer strukturellen Form aus. Teilweise werden davon ausgehend auch Funktionen beschrieben, ohne dass die Arbeit jedoch in diesem Bereich einen Anspruch auf Vollständigkeit erhebt. Diese Methode wird konsequent umgesetzt, auch wenn die Wichtigkeit „funktionaler" Kategorien mit der zunehmender struktureller Komplexität zunimmt. Um trotzdem eine möglichst leserfreundliche Handhabung zu bieten, verweisen „funktionale“ Kategorien im Registerteil auf die Hauptvorkommen der entsprechenden Strukturen. Der Begriff der „funktionalen Kategorie“ wird in der vorliegenden Arbeit verwendet, weil sprachwissenschaftliche Begriffe wie Negation oder Possessivität Usus sind, ohne damit auf generative Ansätze zu verweisen.

An die Hauptarbeit schließt ein „Exkurs“ über konverbale komplexe Prädikate an, die nach Ansicht der Verfasserin mit einer formbasierten Beschreibung nur einseitig und unzureichend dargestellt werden können. Der Exkurs soll damit die Möglichkeit eines Perspektivwechsels eröffnen. Aus dem strukturell kleinteiligen Bild im Falle der Konverben soll mit einer an Funktionen orientierten Betrachtung ein Blick auf das größere Ganze ermöglicht werden. In einem Bereich wird also aus einzelnen Flicken ein Flickenteppich zusammengesetzt.

Um eine Verdopplung von Beschreibungen zu minimieren, werden negierte Sprachbeispiele im jeweils in einem eigenen Unterkapitel im Anschluß an die jeweiligen affirmativen Konstruktionen beschrieben. Die einzelnen Strukturen sind wie folgt in den Rahmen der Arbeit eingebettet:

Kapitel 1 bietet neben einer Einführung in die theoretische Einbettung der Arbeit (1.1) und das zugrundeliegende Sprachkorpus (1.3) auch einen Überblick über die Selkupen und ihre dialektale Vielfalt (1.2). Der bereits angesprochene typologische Abriss (1.6) und eine Sprachprobe (1.6) runden das erste Kapitel ab.

Ein Überblick über die unterschiedlichen Phrasentypen ist in Kapitel 2 verortet. Hier werden detailliert die im Selkupischen verwendeten Phrasentypen und ihre jeweiligen Modifikatoren anhand von Beispielen dargestellt. $\mathrm{Zu}$ den in den Beispielen belegten Phrasen gehören neben Nominalphrasen (2.1) und Verbalphrasen (2.8) auch Adjektivphrasen (2.3), Adverbialphrasen (2.5) und Adpositionalphrasen (2.7).

Auf der Phrasenstruktur aufbauend widmet sich Kapitel 3 den Satzgliedern im einfachen Satz, dessen Modi in Kapitel 4 detailliert beschrieben werden. Im Selkupischen sind das Deklarativsätze (4.1), Interrogativsätze (4.2) sowie Imperativsätze (4.3). 
Mit komplexen Sätzen schließlich beschäftigt sich Kapitel 5. Betrachtet werden unterschiedliche syntaktische Mittel, um die traditionellen Konzepte der Koordination (5.1) und Subordination (5.2) wiederzugeben. Hierzu zählen neben der Juxtaposition vor allem infinite Verbformen sowie aus dem Russischen eptlehnte Konjunktionen. Im Rahmen der Subordination werden Komplementsätze (5.2.3.1), Adverbialsätze (5.2.3.2) und Relativsätze (5.2.4) betrachtet.

Das oben eingeführte Exkurs-Kapitel wird in Kapitel 6 (Konverbale Prädikate) betrachtet. Anhand von Belegbeispielen wird beschrieben, mit welchen Strukturen die funktionalen Kategorien umgesetzt werden. Ergänzend wird der Blick sowohl auf das Nordselkupische als auch auf andere samojedische bzw. uralische Sprachen oder Turksprachen gerichtet, um so ein detailliertes Bild zu erhalten.

\subsection{Theoretische Orientierung}

Die vorliegende syntaktische Beschreibung orientiert sich an den grundlegenden theoretischen Konzepten, „that underlie all work in language description and change, and the postulation of general properties of human language ..." (Dixon 1997: 128), die Dixon (1997) erstmals als ,.basic linguistic theory“ (BLT) bezeichnet hat. Die Theorie wurde von ihm (Dixon 2010a, Dixon 2010b, Dixon 2012) und von Dryer (2006a), Dryer (2006b) später als eigenständige linguistische Theorie weiter ausgearbeitet. Die BLT ist dabei das Ergebnis einer jahrzehnte- und jahrhundertelangen Entwicklung während derer Wissen über das Verfassen grammatischer Beschreibungen und über grammatische Studien selbst gesammelt wurde.

Die BLT basiert zu einem wesentlichen Anteil auf traditionellen Grammatiken, übernimmt dabei „as much as possible from earlier traditions and only as much as necessary from new traditions" (Dryer 2006a: 3). Ausgehend von der Betrachtung jeder Sprache „as a complete linguistic system“ (Dixon 2010a: 4) ist es das Ziel der BLT, jede Sprache in ihren eigenen Termini zu beschreiben, anstatt sie an einen vorgegebenen formalen theoretischen Rahmen anzupassen. In dieser dem Strukturalismus entnommenen Methode liegt der größte Unterschied zur traditionellen Grammatik. Gemeinsamkeiten mit generativen Ansätzen finden sich vor allem indirekt über die Sprachtypologie, im Hinblick auf die zu untersuchenden Parameter. Ein direkt übernommenes Konzept ist das der „sekundären Prädikation“. Die BLT verändert sich fortlaufend, vor allem durch die gegenwärtigen typologischen und formal-linguistischen Entwicklungen (Evans/Dench 2006, Rice 2006b, Rice 2006a, Dixon 2010a: 3).

Auch wenn die BLT nicht versucht, Sprachen in einen theoretischen Rahmen einzupassen, verwendet sie doch theoretische Begriffe, soweit sie zur Beschreibung einer Sprache geeignet und hilfreich sind. Hierunter fallen unter anderem Konzepte wie Subjekt oder Objekt, aber auch das Konzept des Relativsatzes oder der Negation (vgl. hierzu auch Dixon 1997: 128).

${ }^{1}$ Dixon führt die Entwicklung sogar bis zu den Anfängen grammatischer Betrachtungen überhaupt zurück, auf den altindischen Grammatiker Panini, der im 5. Jh. v. Chr. gelebt hat, und auf die vorchristliche griechische Philosophie (Dixon 1997: 128). 
Die Entscheidung, die BLT als Rahmen für die voliegende Arbeit zu verwenden, beruht auf verschiedenen Aspekten: Das Selkupische, und hier insbesondere die zentralen und südlichen Dialekte, sind in verschiedenen Bereichen bisher nur wenig dokumentiert, innerhalb der Syntax gibt es darüber hinaus noch etliche wenig erforschte Bereiche. Die Verfasserin ist daher der Ansicht, dass soviel theoretischer Rahmen wie nötig und so wenig wie möglich verwendet werden sollte. Überdies ermöglicht die BLT eine induktive Arbeitsweise, da ihr Augenmerk entsprechend weit gefasst ist. Zusätzlich soll der Gefahr einer einschränkenden (indoeuropäischen) Sprachbetrachtung, bedingt auch durch den kulturellen und gesellschaftlichen Kontext der Verfasserin, vorgebeugt werden, indem mittels der BLT der Fokus erweitert wird und so Strukturen aufgedeckt werden können, die innerhalb des „heimischen Blicks“ nicht zu erwarten sind.

Für eine bessere Handhabung werden sowohl in der Arbeit als auch im Stichwortverzeichnis Begriffe verwendet, die aus dem funktionalen Bereich stammen. Es ist der Wunsch der Verfasserin, dass die Arbeit auch bei konsequenter Ausrichtung an der Struktur ihre Lesbarkeit behält.

\subsection{Das Selkupische}

Das Selkupische (veraltet Ostjak-Samojedisch) ist der einzige noch lebende Vetreter des südsamojedischen Zweiges. Zusammen mit dem nordsamojedischen und dem finnougrischen Zweig bildet er die uralische Sprachfamilie (Janhunen 1998: 459). Die nächstverwandte Sprache ist das am Ende des 20. Jahrhunderts ausgestorbene Kamassisch (vgl. ebd.). Zum nordsamojedischen Zweig werden das Nenzische (veraltet Juraksamojedisch), das Enzische (veraltet Jenissej-Samojedisch) und das Nganasanische (veraltet Tawgy-Samojedisch) gezählt.

Die Selkupen siedeln weit verstreut in Sibirien zwischen den Flüssen Ob und Jenissej. Seit dem 17. Jahrhundert teilen sie sich in zwei ethnische Gruppen: Ein Teil lebt in den nördlicheren Gebieten Sibiriens, entlang der Flüsse Taz, Turuchan und Jenissej und formt heute das nordselkupische Dialektkontinuum. Eine zweite Gruppe verblieb weiter südlich, im ursprünglichen Siedlungsgebiet, entlang des Flusses Ob und seiner Nebenflüsse Tschulym, Chaja, Ket', Parabel', Tym und Vasjugan (Sobanski 2001: 171). Aus dieser Gruppe bildeten sich die zentralen (Parabel', Tym, Vasjugan) und südlichen (Ob, Chulym, Chaja, Ket') Dialektgruppen heraus. Abbildung 1 zeigt die Siedlungsgebiete der heutigen Selkupen. Laut Angaben der letzten russischen Volkszählung von 2010 leben insgesamt noch 3.649 Selkupen in Russland, 1.023 Menschen haben angegeben, dass sie aktive Sprecher des Selkupischen sind. Allerdings ist die Verteilung der Sprecher nicht gleichmäßig. Die Ergebnisse neuerer Feldforschungen zeigen, dass die meisten Sprecher eine Varietät des Norddialektes sprechen, während das Süd- und das Zentralselkupische extrem vom Aussterben bedroht sind. Nach aktuellen Schätzungen gibt es in den beiden letztgenannten Dialektgruppen nur noch einzelne aktive Sprecher.

Eine wesentliche Ursache dafür, dass die große Mehrheit der noch lebenden Selku- 


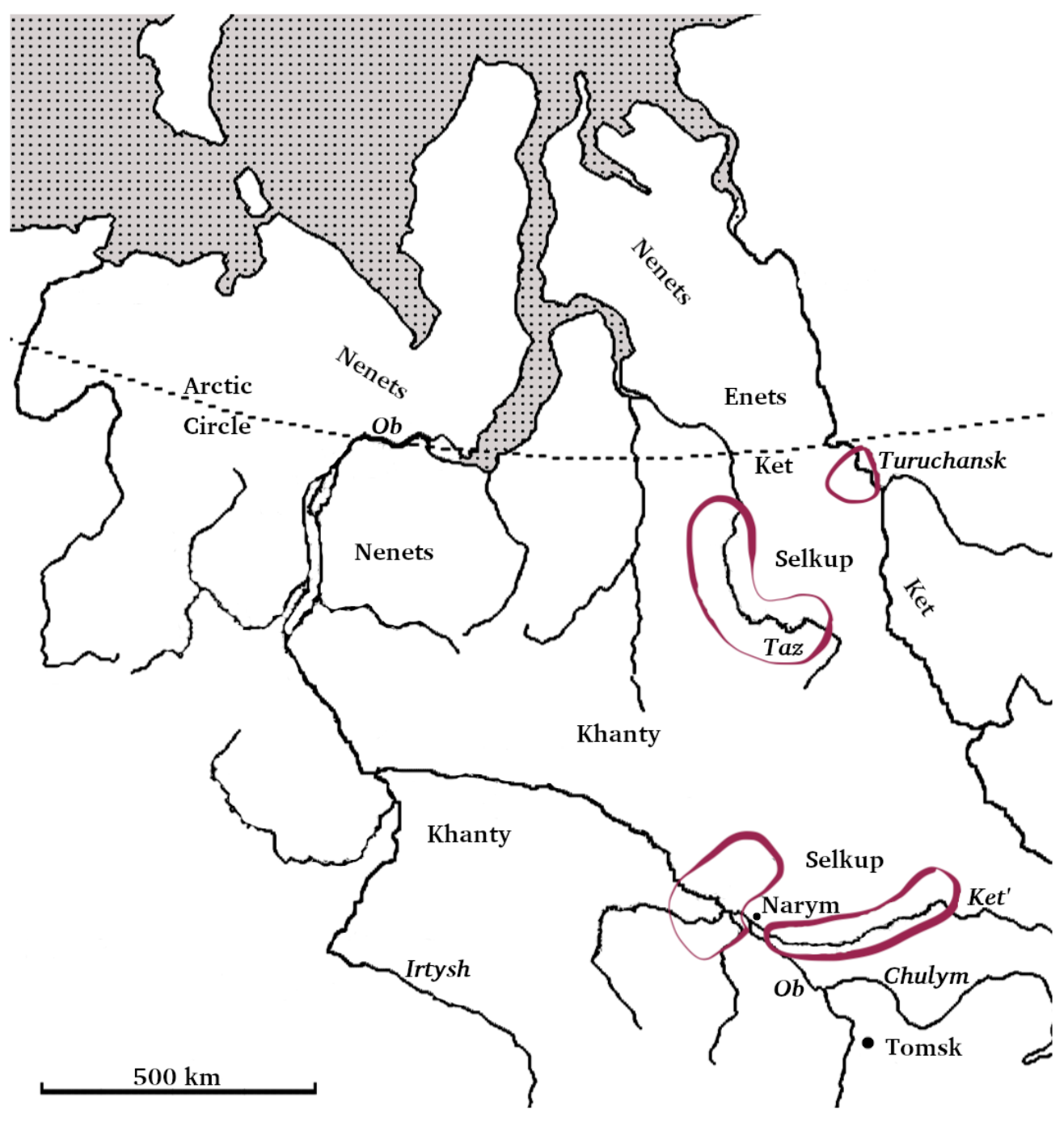

Abb. 1: Siedlungsgebiete der Selkupen

pischsprecher eine Varietät des Nordselkupischen spricht, ist die russische Expansion, die in den südlichen Gebieten sowohl früher begann als auch hier deutlich intensiver in ihrer Auswirkung war. Die in den nördlichen Gebieten beheimateten Selkupen konnten deshalb ihre traditionelle Lebensform und damit auch ihre Sprache länger bewahren als die Selkupen der Südgebiete. Zwischen den beiden Gruppierungen der Selkupen bestehen seit längerer Zeit keine unmittelbaren Kontakte mehr, was dazu führte, dass die Sprachen sich divergierend entwickelten und eine gegenseitige Verständigung heute kaum mehr möglich ist. Damit sind die zwei Gruppen sowohl kulturell als auch sprachlich sehr unterschiedlich.

Das Selkupische weist eine enorme dialektale Vielfalt auf. Über die Einteilung derselben herrscht bis heute keine Einigkeit unter den Forschern. Je nach Forscher wird von drei (z. B. Glushkov/Bajdak/Maksimova 2011), vier oder mehr Dialektgruppen 
(z. B. Alatalo 1994, Alatalo/Donner/Sirelius 2004, Helimski 1998) ausgegangen. So betrachtet Helimski (1998) den Ket'-Dialekt als eigenständige dialektale Gruppe.

Die vorliegende Arbeit orientiert sich bei der dialektalen Einteilung an Gluškov (vgl. Glushkov/Bajdak/Maksimova 2011). Demnach gliedert sich das Selkupische in drei größere dialektale Gruppen: Nordselkupisch, Zentralselkupisch und Südselkupisch, die sich jeweils in weitere Unterdialekte aufteilen. Ethnografisch gesehen lassen sich nur zwei Gruppen unterscheiden, die Nordselkupen und die Nicht-Nordselkupen (kurz Südselkupen) (vgl. Sokolova 1970: 145). Tabelle 1 gibt einen Überblick über die Dialekte und Subdialekte2. Nicht erfasst sind dort die Vielzahl der Mundarten.

Tab. 1: Dialekte des Selkupischen

\begin{tabular}{lll}
\hline Nord & Zentral & Süd \\
\hline Taz & Tym & Mittlerer Ob \\
Tolka (Larjak) & Narym & Oberer Ob \\
Karasino & Vasjugan & Chaja \\
Turuchan & Vakh & Chulym (†) \\
Baicha & & Ket' \\
Eloguj & & \\
\hline
\end{tabular}

\subsection{Sprachdaten}

Grundlage der Beschreibung und der Textanalysen bildet das im Projekt „Syntaktische Beschreibung der süd- und zentralselkupischen Dialekte: Eine korpusbasierte Untersuchung “ 3 erstellte digitale Sprachkorpus, das am Hamburger Zentrum für Sprachkorpora archiviert ist (Budzisch/Harder/Wagner-Nagy 20194). Das Korpus gibt die gesprochene selkupische Sprache aus einem Zeitraum von etwa 150 Jahren in schriftlich fixierter Form wieder. Insgesamt beinhaltet es 144 bereits publizierte Texte mit 9.257 Äußerungen und 53.806 Tokens. Die Texte stammen aus unterschiedlichen nord-, zentral- und südselkupischen Dialekten sowie einem Mischdialekt, der sowohl Merkmale der zentralen als auch der südlichen Dialekte aufweist (Glushkov/Bajdak (2016)). Enthalten sind Sprachdaten von 53 unterschiedlichen Sprechern, die zwischen 1855 und 2015 publiziert wurden. Die Geburtsdaten der Sprecher, soweit sie in den Metadaten erfasst sind, umfassen den Zeitraum zwischen 1890 und 19675. Unter den Informanten sind etwa ein Drittel mehr Frauen (28) als Männer (20) zu finden. Im Korpus befinden sich folkloristische (flk)

\footnotetext{
${ }^{2}$ Innerhalb des Ket'-Dialektes lassen sich die Subdialekte Nieder Ket', Mittlerer Ket' und Oberer Ket' unterscheiden.

${ }^{3}$ Das Projekt wurde im Zeitraum 2015-2018 von der Deutschen Forschungsgemeinschaft (DFG) unter der Nummer WA 3153/3-1 finanziert.

${ }^{4}$ Der Zugang zum Korpus ist über http://hdl.handle.net/11022/0000-0007-D009-4 möglich.

${ }^{5} \mathrm{Zu}$ den Heldenliedern von 1845 sind hinsichtlich der Sprecher keine Metadaten beschrieben
} 
und narrative (nar) Texte sowie heroische Lieder (song) selkupischen Ursprungs 6 und einige Übersetzungen aus dem Russischen oder aus einem selkupischen Dialekt in einen anderen Dialekt (trans). Die ältesten Aufzeichnungen im Korpus stammen aus dem Jahr 1845, die jüngsten aus 2014. Die überwiegende Anzahl der Texte stammt aus den 1960er- (52 Texte), 1970er- (31 Texte) und aus den 1980erJahren (29 Texte). Damit eignet sich das Korpus nicht nur für synchrone, sondern bedingt auch für diachrone Untersuchungen. Eine eindeutige Lokalisierung der hier verwendeten Textbeispiele im Selkup Language Corpus ist über die individuelle Textbezeichnung möglich, die sich nach dem Muster Name_Jahr_Text_Genre zusammensetzt. Der (Sprecher-) Name setzt sich zusammen aus den Initialen des Nachnamens, des Patronyms und des Vornamens. Es folgt das Jahr der Textaufnahme, ein kurzer englischer Titel sowie die Abkürzung des Genre (flk $=$ folklore, nar $=$ narrative, song $=$ Heldenlied, trans $=$ translation). In der vorliegenden Arbeit wird den Beipielen zusätzlich die entsprechende Verortung im Text sowie die abgekürzte Dialektgruppe $(\mathrm{N}=$ Nord, $\mathrm{Z}=$ Zentral, $\mathrm{S}=$ Süd) und der Subdialekt hinzugefügt. Eine Bezeichnung könnte wie folgt aussehen: (ILP_1981_StonyOldWoman_flk_3, S: Middle Ob).

Tabelle 2 zeigt die Verteilung der Texte auf die selkupischen Dialekte und die Genre. Eine detaillierte Aufstellung, die auch die Verteilung der Texte auf die einzelnen (Sub-)Dialekte berücksichtigt, befindet sich in Anhang B.

Um sowohl Nachvollziehbarkeit als auch Einheitlichkeit zu gewährleisten, wird das

Tab. 2: Materialbasis

\begin{tabular}{l|rrrrr}
\hline Dialektgruppe & Gesamt & flk & nar & trans & song \\
\hline Nord & 26 & 26 & & & \\
Zentral & 48 & 28 & 12 & 9 & \\
Zentral-Süd & 4 & & & & 4 \\
Süd & 66 & 52 & 5 & 12 & \\
\hline Gesamt & 144 & 106 & 17 & 21 & 4 \\
\hline
\end{tabular}

im zugrunde liegenden Selkup Language Corpus erstellte und dort verwendete Grapheminventar zur orthographischen Darstellung der Beispiele benutzt. Zudem spielt die Ebene der Phoneme und ihre Realisierungen auf orthographischer Ebene für die vorliegende Arbeit eine untergeordnete Rolle. Die verwendeten Grapheme sind für einen leichteren Vergleich mit den originalen Sprachmaterialien in Anhang A aufgelistet. Abweichend hiervon wird in der vorliegenden Arbeit zur Kennzeichnung der Vokallänge ein Makron (ā) anstelle des IPA-Symbols (a:) über dem entsprechenden Vokal verwendet. Ebenfalls aus dem Selkup Language Corpus übernommen wird die durchgängig englische Glossierung in der Darstellung der Sprachbeispiele. Einige lexikographische Festlegungen gehören ebenfalls dazu: Bei der Darstellung von Verben wird durchgängig der Verbstamm angegeben (pakti - 'springen'), unmarkierte Formen sind bei der Deklination im Numerus der Singular, bei den Kasus der Nominativ.

\footnotetext{
${ }^{6}$ Die Heldenlieder wurden von Alexander Castrén in der Mitte des 19. Jh. gesammelt.
} 


\subsection{Methodik}

Ausgehend von dem in Kapitel 1 genannten Ziel der vorliegenden Arbeit, die syntaktischen Strukturen des Selkupischen auf der Grundlage eines Sprachkorpus zu beschreiben, ergibt sich auch die Herangehensweise. Zum einen zeichnet sich die Arbeit durch einen deskriptiven Charakter aus, zum anderen werden im Sinne einer modernen Dokumentationslinguistik (vgl. Gippert/Himmelmann/Mosel 2006, Woodbury 2003, Himmelmann 1998 etc.) die beschriebenen Strukturen und Phänomene konsequent mit Belegstellen im verwendeten Textkorpus verifiziert. Mit der BLT als theoretischem Rahmen werden in der vorliegenden Arbeit keine bestehenden theoretischen Forschungsansätze überprüft, vielmehr ist die mikroskopische Ebene Ausgangspunkt der Betrachtungen. Die Verfasserin ist bemüht, zu jedem beschriebenen Phänomen jeweils mindestens ein Beispiel aus einem zentralen und einem südlichen Dialekt aufzuführen, ggf. auch aus nördlichen Dialekten. Wo es signifikante Unterschiede auch innerhalb der Subdialekte gibt, kann auch mehr als ein Beispiel pro Dialektgruppe erscheinen. Jedes Kapitel beginnt mit einer kurzen Einführung in die Struktur und endet mit einer Zusammenfassung, die auf das folgende Kapitel überleitet. Wo es notwendig oder wichtig erscheint, erfolgt auch ein kurzer Bezug zu entsprechenden nordselkupischen oder samojedischen Strukturen.

Für die Analyse und Auswertung werden verschiedene Software Tools aus dem EXMARaLDA-System verwendet. EXMARaLDA ist ein System für das computergestützte Arbeiten mit (vor allem) mündlichen Korpora. Es besteht aus einem Transkriptions- und Annotationseditor (Partitur-Editor), einem Tool zum Verwalten von Korpora (Corpus-Manager) und einem Such- und Analysewerkzeug (EXAKT). Die Transkriptionen und Annotationen wurden im Partitor-Editor vorgenommen. Alle Texte enthalten eine englische, die meisten Texte auch eine russische und deutsche Übersetzung. Sämtliche Sprachbeispiele enthalten Annotationen zu den syntaktischen Funktionen (SyF) sowie semantischen Rollen (SeR). Darüberhinaus gibt es für einige Texte zusätzliche Annotationen. Abbildung 2 zeigt ein typisches selkupisches Textbeispiel im Partitur-Editor mit den verschiedenen Transkriptionsbzw. Annotationszeilen. Hier sind auch Annotationen zu Existential-, Possessiv- und Lokativsätzen (ExLocPoss) sowie zu Konverben (CVB) enthalten.

${ }^{7}$ Weitere Informationen unter https://exmaralda.org/de/ 


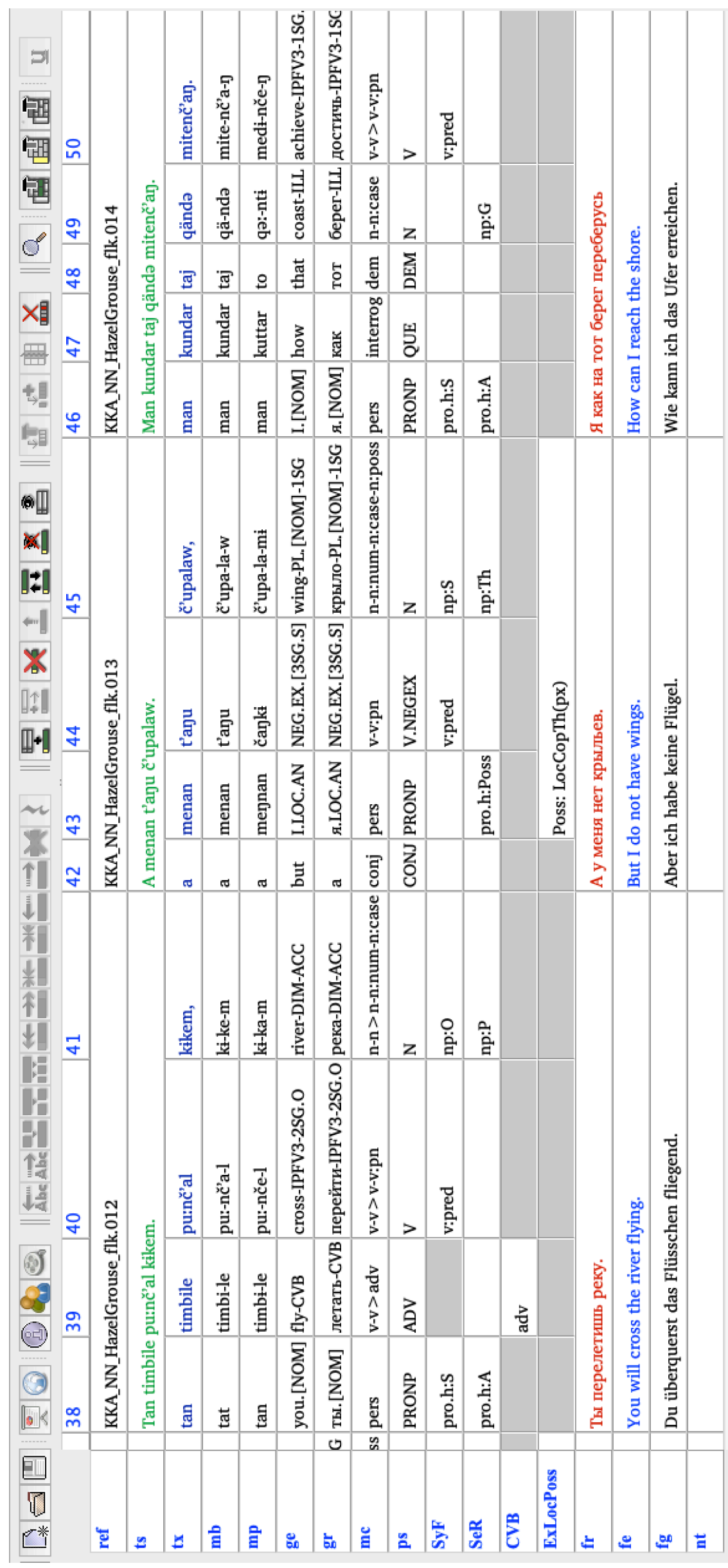

Abb. 2: Textbeispiel im Partitur-Editor inkl. Transkriptions- und Annotationszeilen 
Das mit dem Korpus-Manager Coma zusammengestellte Korpus kann jederzeit mit dem Such- und Analysewerkzeug EXAKT analysiert werden (vgl. Schmidt/Wörner (2005), Wörner (2010). EXAKT ermöglicht die Suche nach (syntaktischen) Phänomenen auf verschiedenen Ebenen: Die Suchanfragen können sich entweder auf transkribiertes Material (Transkriptionssuche), auf Beschreibungen (Deskriptionssuche) oder auf Annotationen beziehen (Annotationssuche). Darüberhinaus können auch Metadaten in die Suche mit einbezogen werden. Die Suchergebnisse können dabei in ihrem jeweiligen Kontext ausgewertet werden.

Abbildung 3 zeigt das Suchergebnis eines aus einfachen Zeichenketten bestehenden Suchausdrucks in Form einer KWiC8-Konkordanz. Der Suchausdruck wird hierbei innerhalb des Kontextes der Fundstelle angegeben. Bei einer Markierung der einzelnen Suchergebnisse werden die entsprechenden Transkriptionen im Partitur-Editor eingeblendet.

\footnotetext{
${ }^{8}$ Key Word in Context
} 


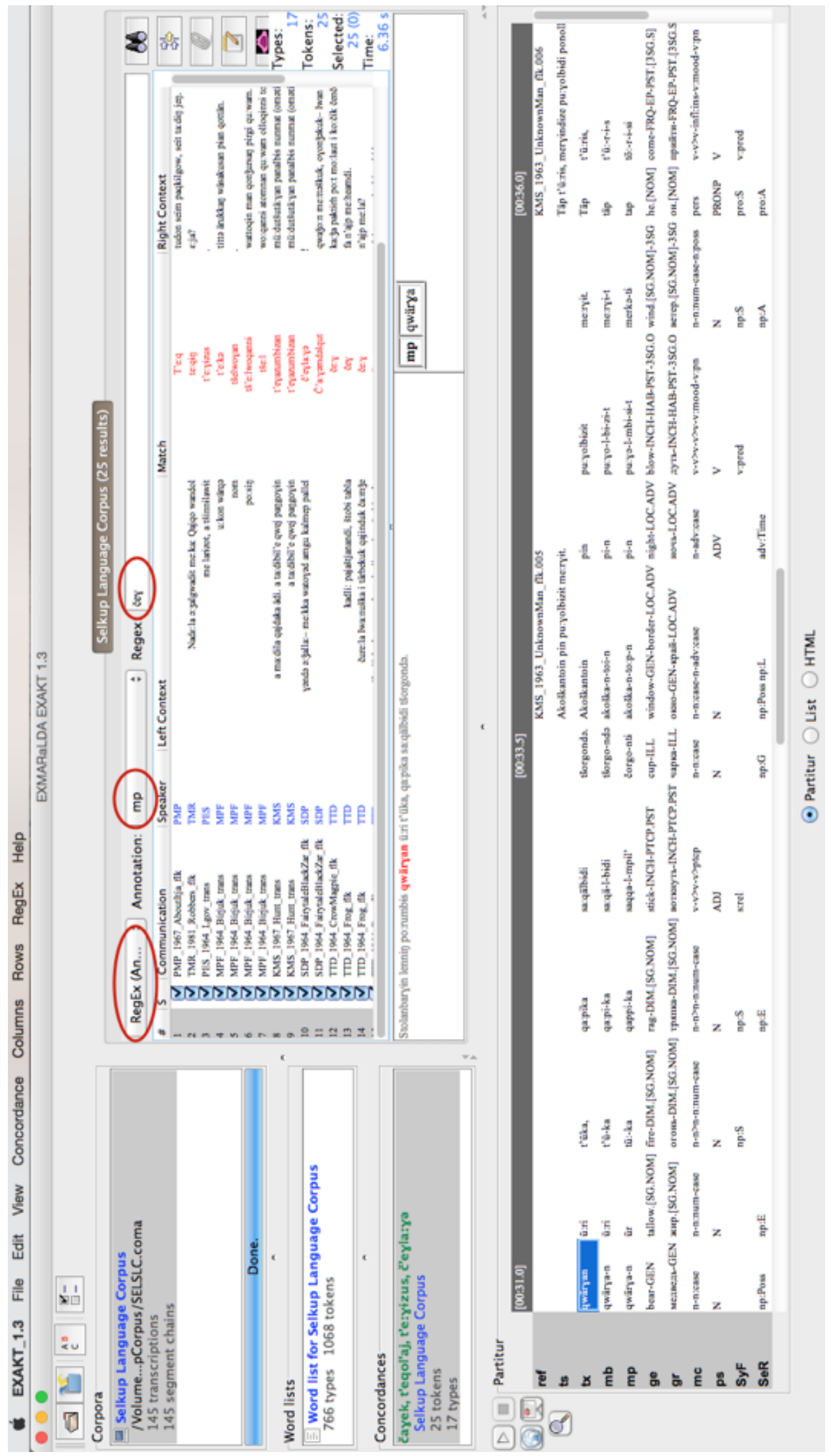

Abb. 3: Korpussuche in EXAKT: Annotationssuche mittels einfacher Zeichenketten, Suchergebnis als KWIC-Konkordanz dargestellt. 
Eine weitere Möglichkeit der Korpussuche bildet die Suche mit Hilfe von regulären Ausdrücken. Auch hier werden die Suchergebnisse als KWIC-Konkordanz dargestellt. Mit Hilfe regulärer Ausdrücke lassen sich auch komplexere Suchanfragen stellen. In Abbildung 4 wurden mittels des regulären Ausdrucks „čey $\backslash b$ “ sämtliche Stellen im Korpus gesucht, in denen das lexikalische Wort „čey“ vorkommt. 


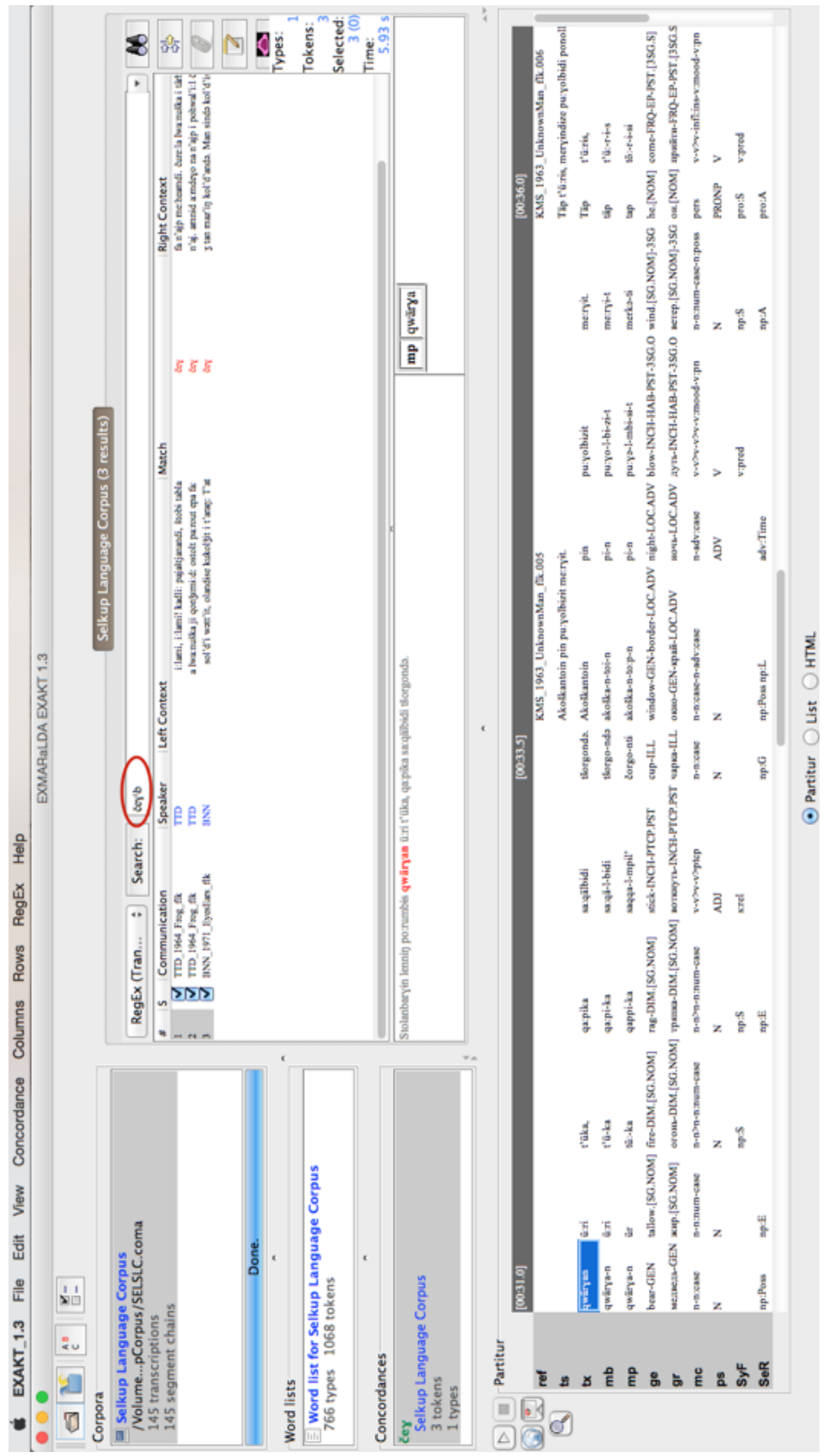

Abb. 4: Korpussuche in EXAKT: Suchausdruck mittels regulärer Ausdrücke, Suchergebnis als KWIC-Konkordanz dargestellt. 


\subsection{Forschungsstand}

Die Anfänge der Grammatikbeschreibung des Selkupischen lassen sich im 19. Jahrhundert verorten. Von Castrén (1854)) stammt die erste Arbeit, in der neben den anderen samojedischen Sprachen auch das Selkupische betrachtet wird. Die nächste grammatische Beschreibung der selkupischen Sprache erfolgte dann erst beinahe 100 Jahre später: In den 1930er Jahren beschäftigte sich G. N. Prokof'jev intensiv mit der Sprache. Seine Werke (Prokof'ev 1931, Prokof'ev 1935, Prokof'ev 1937) bilden auch heute noch einen guten Ausgangspunkt für die Erforschung der selkupischen Sprache, obwohl seine Arbeiten tendenziell kurz sind und auf den nördlichen Dialekten basieren. Nach G. N. Prokof'jev veröffentlichen A.I. Kuznecova/Chelimskij/Grushkina (1980) 50 Jahre später eine moderne, deskriptive Grammatik. Die Arbeit beruht auf Feldforschungsmaterialien, die die Verfasser in den 1970er Jahren gesammelt hatten. Diese Grammatik bleibt leider bis heute die einzige Vollgrammatik, die mehrere Aspekte der Sprache (Phonetik, Phonologie, Morphologie und Syntax) beschreibt. Die überwiegende Mehrheit der grammatischen Abhandlungen enthält lediglich phonologische und morphologische Beschreibungen, die sich zudem größtenteils genau wie A. I. Kuznecovas Arbeit auf die nördlichen Dialekte des Selkupischen stützen.

Die einzige Arbeit, die sich ausschließlich mit dem Thema Syntax auseinandersetzt, ist die syntaktische Beschreibung der samojedischen Sprachen von Tereshchenko (1973). Die Autorin beschäftigt sich mit einfachen Sätzen, wobei sie immer wieder auch selkupische Beispiele angibt, sich hier jedoch vorwiegend auf das Nordselkupische bezieht. Komplexe Sätze fehlen in der Betrachtung, ebenso wie die Beschreibung der Nominalphrasenstrukturen oder der Prädikationstypen.

Mit der Grammatik der Süd- oder Zentraldialekte beschäftigen sich bisher nur wenige Arbeiten: Kuz'mina (1974) sowie Bekker (1995a), Bekker (1995b) beschreiben in ihren Grammatiken hauptsächlich die morphologischen Eigenschaften der südselkupischen Dialekte, eine syntaktische und auch phonologische Untersuchung fehlt hier völlig.

Zu den Grammatiken gehört auch $\mathrm{H}$. Katz' grammatische Beschreibung des TymDialekts (Katz 1975). Nach heutiger Auffassung handelt es sich hierbei um eine korpusbasierte Beschreibung: Der Verfasser hat die von Kai Donner in den 1910er Jahren gesammelten Materialien ausgewertet und anhand dieser Daten eine kurze Grammatik zusammengestellt. Diese beschränkt sich jedoch neben den Wortkommentaren auf eine sehr knappe phonetische Beschreibung. Neben den oben genannten Arbeiten beschäftigen sich einige kleinere Aufsätze mit speziellen Phänomenen des Selkupischen: So beschreibt Alitkina (1985) in ihrem nur vierseitigen Artikel nonverbale Prädikate, wobei sie sich nur auf die Attribution konzentriert, auf weitere Typen (wie Zugehörigkeit (proper inclusion) oder Gleichsetzung (equation)) geht sie nicht ein.

Cheremisina/Martynova (1991) beschreiben die syntaktischen Funktionen des südselkupischen Verbs. Doch auch diese Monografie stellt keine umfassende wissenschaftliche syntaktische Beschreibung dar, fehlen doch wichtige syntaktische 
Bereiche in der Betrachtung, wie etwa die Nominalphrasenstruktur. Obwohl viele Aussagen, die A. I. Kuznecova/Chelimskij/Grushkina (1980) über die Norddialekte getroffen haben, sehr wahrscheinlich auch auf das Süd- und Zentralselkupische übertragen werden können, gibt es in einigen Bereichen erhebliche Unterschiede zwischen den dialektalen Gruppen, die noch genauer erforscht werden sollten.

Die ältesten selkupischen Sprachmaterialien stammen aus dem 18. Jahrhundert. G. F. Müller sammelte als Mitglied der 2. Akademischen Expedition um 1735 vor allem Wortlisten, die leider unveröffentlich blieben. Etwa einhundert Jahre später (am Ende des 19. Jahrhunderts) wurde systematisch damit begonnen, Sprachproben des Selkupischen zu sammeln. Oftmals wurden diese jedoch erst in deutlich später herausgegebenen Textsammlungen veröffentlicht: A. Castrén, gesammelt 1845-1849, veröffentlicht in Castrén (1854), Castrén (1855) (lexikologische und grammatische Materialien) und Castrén/Lehtisalo (1940) (Textsammlungen); N. P. Grigorovskij (1879) (dessen „Die erste sölkupische Fibel“ das erste auf Selkupisch publizierte Buch überhaupt war), neuveröffentlicht in Hajdú (1973) und in Katz (1988). K. Donners Text vom Tym-Dialekt wurde erstmal in Hajdú (1968) und später von Katz (1975) veröffentlicht. In den 30er Jahren des 20. Jahrhunderts begannen G. N. Prokof'jev und seine Frau E. D. Prokof'jeva, die samojedischen Sprachen intensiv zu erforschen. Die Ergebnisse ihrer Arbeit finden sich in zahlreichen Publikationen, unter anderem in einer Grammatik des (Nord)Selkupischen (Prokof'ev 1935) oder in Schulbüchern (Prokof'ev/Prokof'eva 1940, Prokof'eva 1932, Prokof'eva 1953).

Insbesondere für die südselkupischen Dialekte ist die sogenannte Tomsker Schule wichtig. In zahlreichen Publikationen (Dul'zon 1966a, Dul'zon 1966b, Dul'zon/ Porotova 1976, Dul'zon/Porotova 1980, Dul'zon/Porotova 1980, Kuz'mina 1967, Kuz'mina 1974) wurden Texte aus den süd- oder zentralselkupischen Dialekten publiziert. Sie stammen von Feldforschungen, die Andreas Dul'zon und seine Schüler ab den 1960er Jahren durchgeführt habsen. Einige Textsammlungen wurden in den 1980er Jahren auch in der Reihe „Skazki narodov sibirskogo severa“ veröffentlicht. Die Texte, die von Dul'zon oder seinen Schüler herausgegeben wurden, sind in einer phonetischen Transkription verfasst, die jedoch inkonsequent ist, wie bereits einige Forscher (so zum Beispiel Katz (1975: IV)) angegeben haben. Diese Tatsache erschwert die Bearbeitung der Texte, macht sie aber nicht unmöglich.

Zahlreiche Texte blieben lange Zeit unveröffentlicht, mit ihrer Publikation wurde erst in den letzten Jahren begonnen (wie z. B. in Bajdak/Tuchkova (2004) oder Tuchkova/Helimski (2010)). Diese Textveröffentlichungen sind hinsichtlich der Qualität der Transkription konsequenter und zuverlässiger.

\subsection{Typologischer Abriss}

Das Selkupische weist eine überwiegend agglutinierende Morphologie auf. Fusionale Züge finden sich etwa im Paradigma der possessiven Deklination. Unter russischem Einfluss entwickel(te)n sich analytische Konstruktionen, wie beispielsweise der Ge- 
brauch von Präpositionen oder Konjunktionen (Bekker 1995a: 290). Aufgrund des komplexen morphophonologischen Systems (Alternations- und Assimilationsprozesse) werden Suffixe an der Oberfläche unterschiedlich realisiert 9 . Analog zu dem in uralischen Sprachen verbreiteten System von Verbalableitungen (Verbalnomen, Partizipien, Konverben), verfügt das Selkupische über morphologische Mittel für die wechselseitige Transformation der syntaktischen Relationen von nominalen, verbalen und Adjektivstämmen: qum - 'Mann, Mensch' - qumååk - 'Ich bin ein Mensch' - qumilj 'menschlich (ADJ)' - qumik - 'menschlich (ADV)' (Helimski (1998: 557)).

Selkupische Nomen flektieren nach Kasus und Numerus. Darüber hinaus können sie auch verbal flektiert werden und fungieren dann als Prädikat des Satzes (vgl. ebd.) wie in (3a) und (3b).

(3) a. Tan ara-ma-nd. 2SG old-thing-2sG.S

'Du bist ein Alter.' (ChDN_1983_HeroesDaughter_flk_36, Vasjugan)

b. Ax, tan qator-o-nd!

INTERJ 2SG prisoner-EP-2SG.S

'Ach, du bist ein Gefangener.'

(PMP_1967_AboutItja_flk_100, Middle Ob)

Das Selkupische kennt 4 Numeri: Singular, Dual, Plural und Kollektiv. Der Singular ist unmarkiert, zur Kennzeichnung des Plurals werden zwei Suffixe verwendet: $-t$ und $-l a^{10}$. Im Nordselkupischen erscheint ausschließlich $-t$, in den zentralen Dialekten kommen beide Varianten vor, im Südselkupischen praktisch nur -la (zur Verteilung der Suffixe auch Bekker (1995a: 64)). Der Dual trägt das Suffix -qü. Für das Nordselkupische ist der Kollektiv auf -lmi belegt (vgl. Helimski 1998). In den zentralen Dialekten wird er nicht, in den südlichen Dialekten kaum verwendet.

Mittels Possessivsuffixen werden Person und Numerus des Possessors ausgedrückt, in Kombination mit der Kasus- und Numerusflexion des Possessums entsteht ein possessives Deklinationsparadigma. Possessivsuffixe werden darüberhinaus auch zum Ausdruck von Definitheit (vgl. Budzisch (2017b)) verwendet.

Die Anzahl der Kasus variiert in den einzelnen Dialektgruppen, für das Nordselkupische benennt Helimski (1998) 13 Kasus (Nominativ, Genitiv, Akkusativ, Lokativ, Illativ, Ablativ, Prolativ, Dativ/Allativ, Instrumental, Karitiv, Translativ, Koordinativ, Vokativ). Der Vokativ ist im Selkup Language Corpus ebenfalls für das Südselkupische belegt, nicht jedoch für das Zentralselkupische. Der Koordinativ auf -šak ist für das Zentral- und Südselkupische nicht beschrieben (vgl. Bekker (1995a)). Der von Helimski als Koordinativ beschriebene Kasus wird in der vorliegenden Arbeit als Adessiv bezeichnet. Er drückt die Position in der Nähe eines Objektes aus und wird mit

\footnotetext{
${ }^{9}$ Als relevant an dieser Stelle sei die Alternation der Konsonanten $-n|-t|-\varnothing,-m|-p|-\emptyset,-\eta|-k| \emptyset$ genannt

${ }^{10}$ Bekker nimmt an, dass das Suffix -la aus den Turksprachen entlehnt ist, vgl. hierzu auch Bekker (1995a). Eine andere Variante ist, dass der Pluralmarker ein Kognat zum ostseefinnischen Lokativ/Kollektivsuffix -1A ist, wie etwa in finn. kahvi-la - 'Cafe' oder estn. haig-la - 'Krankenhaus' < haige - 'krank'
} 
belebten und unbelebten Nomen verwendet 11 . Das overte Subjekt intransitiver und transitiver Sätze ist identisch markiert, mit dem Nominativ. Damit gehört das Selkupische zu den Nominativ-Akkusativ-Sprachen.

Verben flektieren im Selkupischen nach Person, Numerus, Tempus und Modus. Anders als die nordsamojedischen Sprachen verfügt das Selkupische nicht über ein morphologisches Genus verbi. Es existieren 2 Konjugationstypen, die sog. subjektive und objektive Konjugation, deren Verwendung bis heute nicht endgültig geklärt ist. Neuere Forschungen zeigen einen Zusammenhang zu informationsstrukturellen Faktoren (Wegener 2015). Es werden 4 Tempora unterschieden (Präsens (oder Aorist), Präteritum, Narratives Präteritum, Futur). Analog zu anderen samojedischen Sprachen ist die temporale Bedeutung des Präsens/Aorist vom lexikalischen Aspekt des Verbstamms abhängig. Imperfektive Verben referieren so eher auf eine präsentische Bedeutung, perfektive Verben eher auf die unmittelbare Vergangenheit (Wagner-Nagy 2015: 5). Die Anzahl der (synthetisch gebildeten) Modi variiert innerhalb der Dialektgruppen, es werden zwischen $7^{12}$ und $8^{13}$ Modi unterschieden (Indikativ, Imperativ, Inferential, Auditiv, Konditional, Optativ, Debitiv, Subjunktiv). Vereinzelt ist die Entwicklung von Präverben zu beobachten.

Die Standardnegation verbaler Prädikate erfolgt mittels einer Negationspartikel ( $a$ šša) und der affirmativen Verbform. Das negative Existentialverb čä $j k \dot{i}$ - wird im Nordselkupischen in Präteritalkonstruktionen als Standardnegation verwendet sowie in Existential-, Lokativ- und Possessivsätzen in allen Dialektgruppen. In Imperativsätzen wird die Negationspartikel $i k i$ verwendet. Hinzu kommen die aus dem Russischen entlehnten Negationspartikeln n'etu, n’i (vgl. auch Wagner-Nagy (ebd.)).

Das ursprünglich reichhaltige Inventar infiniter Verbformen, die unter anderem zur Bildung komplexer Sätze verwendet werden ${ }^{14}$, ist vor allem in den zentralen und südlichen Dialekten zunehmend durch die Verwendung subordinierter Sätze mit Konjunktionen nach russischem Muster ersetzt worden (Helimski 1998)15. Konverben können im Selkupischen (vor allem in den südlichen Dialekten), ähnlich wie im Kamassischen und in Turksprachen (Klumpp 2002. Klumpp 2005a, Klumpp 2005b, Johanson 1971, Schönig 1984, Pischlöger 2001, Anderson 2004a) in Auxiliarverbindungen verwendet werden.

Die grundlegende Wortstellung im Selkupischen ist SOV, jedoch erlauben pragmatische bzw. informationsstrukturelle Faktoren Variationen. Der Modifikator ist dem Kopf konsequent vorangestellt. Als Nominativ-Akkusativ-Sprache werden im Selkupischen die Subjekte transitiver und intransitiver Verben identisch markiert (im Nominativ). Direkte Objekte erscheinen entweder im Akkusativ oder im Nominativ (Dif-

\footnotetext{
${ }^{11}$ Die von Bekker (1995a) beschriebene Verwendung des Kasus ausschließlich mit belebten Beispielen lässt sich anhand von Korpusbeispielen widerlegen (z. B. čōbsr-nan - 'berry-AD)

${ }^{12}$ Südselkupisch (Cheremisina/Martynova 1991)

${ }^{13}$ Nordselkupisch (Helimski 1998: 566)

${ }^{14}$ Für das Nordselkupische benennt Helimski (1998) den Infinitiv, 5 Partizipien - Präsens, Präteritum, Karitiv, Debitiv, Destinativ - 3 Konverben sowie 2 Nomina Actionis

${ }^{15}$ In den zentralen und südlichen Dialekten gibt es folgende infinite Formen: Partizip (Präsens und Präteritum), Konverb, Nomen Instrumentalis, Nomen Actionis, Nomen Abstractum, Infinitiv.
} 
ferentielle Objektmarkierung DOM). In Imperativsätzen und nach Numeralia treten Objekte häufig im Nominativ auf, eindeutige Regeln zur Verwendung existieren jedoch bis heute nicht. Indirekte Objekte werden mit dem Dativ/Allativ markiert.

\section{Sprachprobe}

Die folgende Sprachprobe soll nach dem typologischen Abriss einen (ersten) Eindruck des Selkupischen vermitteln. Der Text aus dem zentralselkupischen Tym-Dialekt gibt die mündliche Sprache wieder. Die Sprachprobe ist zuerst im Originaltext gezeigt. Danach sind die Morphemgrenzen sowie die englischen Glossen und eine deutsche Übersetzung angegeben.

Čēy kā̧̌a paktirh pōt mōlaut i kōčik čenčekuk, a kwere āmdi āryoyit i hoyinžešpa: qajko tat, kāža, kōčik čēnčand? A kāža ỉ̌alguk: tat tärband, mat šēptešpak? Kwعre ǐ̧alguk: kud kōčik čenča, kak tat, nā kōčik šēptešpa.

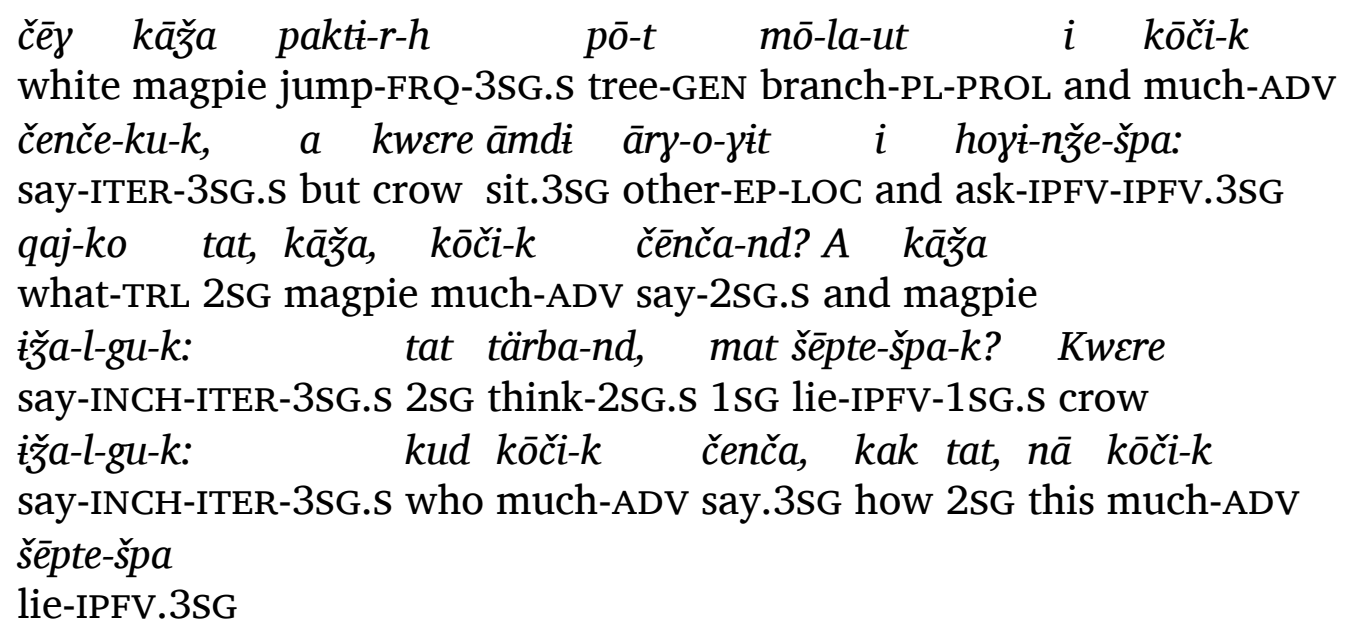

'Eine weiße Elster springt auf den Ästen eines Baumes umher und spricht viel, aber auf einem anderen (Ast) sitzt eine Krähe und fragt: Warum, Elster, sprichst du so viel? Und die Elster sagt: Glaubst du, dass ich lüge? Die Krähe sagt: Wer so viel spricht wie du, der lügt oft.'

(TTD_1964_CrowMagpie_flk, Z: Tym) 


\section{Phrasentypen}

In den zentral- und südselkupischen Dialekten bilden die folgenden fünf Phrasentypen innerhalb einer anderen Phrase oder innerhalb eines Satzes Konstituenten: Nominalphrase (NP), Verbphrase (VP), Adjektivphrase (AP), Adverbphrase (AdvP) und Adpositionalphrase (AdP). Entsprechend dem in Kapitel 1.1 auf Seite 3 eingeführten theoretischen Rahmen werden zur Beschreibung der Phrasenstruktur generative Termini, wie beispielsweise „Phrase“ oder „Konstituente“, verwendet. Auf die Darstellung syntaktischer Bäume wird dagegen verzichtet.

Nominalphrasen, Verbphrasen, Adjektivphrasen und Adverbphrasen sind im Korpus auch negiert belegt. Sie werden jeweils am Ende der affirmativen Phrasen in einem eigenen Unterkapitel beschrieben. Beispiele für negierte Adpositionalphrasen lassen sich nicht belegen.

\subsection{Nominalphrase}

Eine Nominalphrase besteht mindestens aus einer nominalen Komponente, die nach Kasus und Numerus flektiert. Innerhalb der Nominalphrase lassen sich solche unterscheiden, deren Kopf aus einem Nomen besteht, von denen, deren Kopf ein Pronomen ist. Nominalphrasen können als Komplemente aller Phrasentypen fungieren (z.B. Subjekt und Objekt als Komplemente des Verbs) sowie als Adjunkte des Verbs (Adverbiale) und Modifikatoren weiterer NPs (z.B. Possessoren). Auch Relativsätze (Kapitel 5.2.4) und infinite Verbformen (wie Konverbkonstruktionen) können NPs modifizieren. Bis auf wenige Ausnahmen erscheint der Modifikator einer NP pränominal. Zwischen dem Kopfnomen und seinen Attributen gibt es keinerlei Kongruenz. Nominalphrasen folgen in der Regel der Struktur ((Dem) + (andere Mod. $)+$ Nominal + (emph. Pron. $)+($ inf. Verbform $)+($ Rel $))_{\mathrm{NP}}$. Optionale Komponenten befinden sich in Klammern.

\subsubsection{Nominale Köpfe}

$\mathrm{Zu}$ den nominalen Köpfen gehören im Selkupischen Nomen und Pronomen. Nachfolgend sind einige Beispiele und Beschreibungen für mögliche (pro)nominale Köpfe aufgeführt. 


\section{Nomen}

Die kleinstmögliche Nominalphrase ist in den Beispielen (4) - (6) dargestellt. In (4) und (5) sind die Nomen n'adek - 'Mädchen' und àdat - 'Frühling' jeweils Subjekt des Satzes, in (6) ist das Nomen das direkte Objekt (čobir-p - 'Beere-ACC').

(4) $\left[\boldsymbol{N}^{\prime} \text { adek }\right]_{N P}$ tab-i-p kwat-pa-t.

girl 3SG-EP-ACC kill-PST.REP-3SG.O

'Das Mädchen tötete sie.'

(ChDN_1983_GirlAndIce_flk_30, Z: Vasjugan)

(5) $[\bar{\partial} d \partial-t]_{N P} \quad m \bar{e}-t a$.

spring-3SG do-IPFV2.3SG

'Der Frühling kam.'

(TFF_1967_KalabokkaPönegessa_flk_4, S: Middle Ob)

(6) Tab [čobir-p] $]_{N P}$ awē-špa-s

3SG berry-ACC eat-IPFV-PST.3SG

'Er aß Beeren.'

(SEV_1981_SisterBrother_flk_23, S: Middle Ob)

\section{Pronomen}

Die kleinstmögliche pronominale NP enthält mindestens einen pronominalen Kopf. Das Korpus zeigt unterschiedliche pronominale Köpfe: In Beispiel (7a) tritt das Reflexivpronomen onž - 'selbst' als Kopf der Nominalphrase auf, hier ohne Modifikator. Die Beispiele (7b) und (7c) zeigen das Personalpronomen der 1. Person Plural $m$ 'i - '1DU' als pronominalen Kopf, der mit einem Nomen (čumilqummut - 'Tschumylkupl bzw. einem Pronomen² (taz'e - '2sG.COM') modifiziert ist. Beispiel 7d enthält die Indefinitpronomen kudi-koj - 'who-emph' und qai-p-koj - 'WHAT-ACC-EMPH', die als pronominaler Kopf auftraten.

a. Na kibajč kib.and-o-yindi čanni-mba, DEM boy small.boat-EP-EL.3SG go.out-PST.REP.3SG

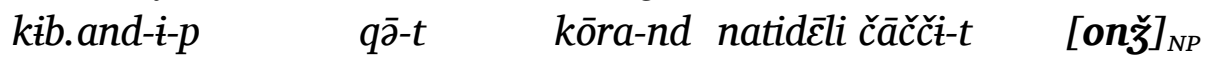
small.boat-EP-ACC coast-GEN sand-ILL there pull-3sG.o himself šöt $\quad$ qwan-ba.

into.forest go.away-PST.NAR.3SG

'Der Junge stieg aus dem Boot aus, zog das Boot auf den Sandstrand und ging selbst in die Taiga.'

(SEV_1981_SisterBrother_flk_22, S: Middle Ob)

\footnotetext{
${ }^{1}$ Tschumylkup ist ein Ethnonym und wird von Selkupen verwendet, die einen der zentralselkupischen Dialekte sprechen.

${ }^{2}$ Die pronominale Konstruktion in $(7 c)$ entspricht der russischen Bildungsweise.
} 
b. [M'i čumil.qumm-ut] $]_{N P}$, nid'i-k mē-kka-ut.

1PL Selkup.person-1PL, such-ADV do-DUR-1PL

'Wir Chumylkupen tun es so.'

(ChDN_1983_MistressOfFire_flk_84, Z: Vasjugan)

c. [Me ta-z'e $]_{N P}$ söd-ə-zun ile-ža-j.

1PL 2SG-COM forest-EP-LOC live-FUT-1DU

'Wir werden zusammen [wrtl.: wir mir dir] im Wald leben.' (PVD_1961_FarmAssault_flk_116, S: Chaja)

d. Üdi-t tab-e-y ünde-di-di: [kudi-koj] $]_{N P 1}$

evening-LOC.ADV 3SG-EP-DU hear-DRV-3DU.O who-EMPH

[qai-p-koj$]_{N P 2}$ l'ane-če-mba-d.

what-ACC-EMPH knock-DRV-PST.REP-3SG.O

'Am Abend hörten sie: Irgendjemand klopft mit irgendetwas.'

(ChDN_1983_Nikita_flk_34, Z: Vasjugan)

\subsubsection{Modifikatoren}

Das nachfolgende Kapitel gibt eine Übersicht über mögliche Modifikatoren, mit denen eine NP im Zentral- und Südselkupischen erweitert werden kann. Hierunter fallen morphosyntaktisch markierte Konstruktionen ebenso wie rein syntaktische markierte. Die morphosyntaktische Markierung (Kasus und Numerus) erfolgt am Kopf, eine Kongruenz zwischen Kopf und Modifikator existiert nicht. Von Modifikatoren einfacher NPs lassen sich Modifikatoren komplexer NPs unterscheiden.

Dixon unterscheidet "Modifiers of a common noun as head of an NP typically include: $[\mathrm{O}]$ ne or more adjectives [..., [A] cardinal or ordinal number or a quantifier [..., [O]ne or more nouns [...]. More complex modifiers of an NP head include: [A] possessive phrase ([T]his is effectively an NP embedded within an NP [...]), [A] relative clause $[\ldots,[\mathrm{A}] \mathrm{n} \mathrm{NP}$, typically referring to spatial or temporal location of the head [...] (Dixon 2010a: 106-108).

In Anlehnung an Dryer (2007: 151ff.) beginnt die Übersicht mit einer Darstellung der formal einfacher Modifikatoren (Pronomen, Adjektive, Numeralia, Quantifizierer, Partizipien), im Anschluss folgt die Beschreibung formal komplexer Modifikatoren (genitivische oder possessive Konstruktionen, multiple Modifikatoren) sowie elliptischer Nominalphrasen.

\subsubsection{Pronomen}

Als Modifikatoren von NPs erscheinen Pronomen im Südselkupischen in Form von Possessiv-, Demonstrativ- und Interrogativpronomen sowie einem emphatischen Pronomen. 


\subsubsection{Possessivpronomen}

Als Modifikator einer NP ist das Personalpronomen immer ein Possessivpronomen. Es erscheint stets pränominal im Adessiv oder Nominativ (unmarkierte Form) auf. Bedingt durch die Alternation $t \sim n$ erscheinen in der ersten und zweiten Person Singular sowohl die Formen mat (10a) als auch man (10b), wobei in den zentralen Dialekten vor allem die Variante mat auftritt, in den südlichen Dialekten hingegen man. In beiden Dialektgruppen kann eine zusätzliche Possessivitätsmarkierung (PX) am Kopfnomen erfolgen. Korpusanalysen zeigen, dass dies für belebte Nomen (inkl. Verwandtschaftsbeziehungen), Körperteile sowie für Objekte und Abstrakta zutrifft, mit denen eine enge Zugehörigkeit ausgedrückt werden soll (vgl. hierfür auch Stassens Beschreibung der „inalienable possession“, die praktisch deckungsgleich ist (Stassen 2001: 954).Im Gegenzug ist das Kopfnomen bei der „alienable possession“ unmarkiert. Tabelle 3 zeigt die entsprechende Verteilung im Korpus. Die Korpusdaten

Tab. 3: Possessivpronomen 1. Person und PX-Markierung des Kopfnomens

\begin{tabular}{l|ll} 
& PX-markiert & unmarked \\
\hline "alienable“ & 1 & 33 \\
„inalienable“ & 164 & 21 \\
\hline
\end{tabular}

zeigen, dass das System vor allem im Bereich der „alienable possession“ in beiden Dialektgruppen intakt ist. Im Bereich der „inalienable possession“ zeigt das System Defekte. Insbesondere in übersetzten Texten aus dem Russischen treten hier teilweise unmarkierte Kopfnomen auf, wie im zentralselkupischen Beispiel (8).

(8) [Mat tōbe $]_{N P}$ č'el'čo-

1sG leg press-AOR.3sG

'Ich habe meinen Fuß (auf sie) gestellt.'

(SDP_1964_FairytaleBlackZar_flk_468, Z: Narym)

Die zentralselkupischen Beispiele (9a) und (9b) zeigen Kopfnomen, die Verwandtschaftsbeziehungen ausdrücken ( $p a j a$ - 'Frau' bzw. ă̌'uka - 'Großmutter'). Sie sind jeweils mit dem Possessivsuffix der 1. Person Singular - $m$ markiert.

Das südselkupische Beispiel (10a) zeigt die Nominalphrase matp'argem - 'mein BauchACC', in der das unmarkierte Possessivpronomen der ersten Person Singular mat 'mein' verwendet wird. Die Markierung $-m$ am Kopfnomen kann sowohl ACC als auch eine Possessivsuffix der 1. Person Singular sein (1SG, die Glosse ist daher 1SG.NOM/ACC sein. Das das Kopfnomen p'arge ein Körperteil bezeichnet, ist ein Nominativobjekt mit Possessivmarkierung durchaus denkbar. In Beispiel (10b) erscheint das Possessivpronomen der ersten Person Singular in der Form man, zusätzlich markiert das Possessivsuffix - $w$ - am Kopfnomen era - 'Ehemann' die Possessivität.
a. $N \bar{a} \quad[\text { mat paja- } m]_{N P}$
tö-špi-nda.
DEM $1 G$
old.woman-1SG come-IPFV-INFER.3SG 
'Das ist meine Frau, die kommt.'

(TTD_1964_Frog_flk_56, Z: Tym)

b. [Man ă̌uka-m] $]_{N P}$ ugot tabe-č-le kwaja-kku-mba.

1SG grandmother-1SG earlier squirrel-CAP-CVB go-ITER-PST.REP.3SG

'Meine Großmutter ging früher Eichhörnchen jagen.'

(SAA_1984_MyGrandmother_flk_1, Z: Narym)

a. Tan [mat p'arge-m] $]_{N P} \quad$ og poža-ľ̆-le, mat

2SG 1SG stomach-NOM/ACC.1SG NEG.IMP unpick-TR-2SG.O, 1SG

kwēsse tašt töd-la-k-s.

back 2sG.ACC shit-OPT-1SG.S-CLIT

'Schneide meinen Bauch nicht auf, ich kotze (scheiße) dich wieder aus.' (ILP_1981_ItjaPönegesse_flk_11, S: Middle Ob)

b. Aaa, [man era-w-ne $]_{N P}$.

INTERJ, 1SG husband-1SG-DAT

'Aaa, zu meinem Mann.'

(PMP_1961_Fairytale_flk_152, S: Upper Ob)

In beiden Dialektgruppen erscheint man außer in der unmarkierten Form auch im Adessiv. Hierbei ist in den Formen teilweise das Kasussuffix mit dem Possessivpronomen verschmolzen wie in den Beispielen (11a) - (11d). 3. Beispiel (11c) ist eine 1:1-Übersetzung aus dem Russischen, inklusive der Relativsatzkonstruktion mit dem russischen Interrogativpronomen što - 'was'. In den Beispielen (11a), (11d) und (11e) erfolgt eine Possessivmarkierung auch am Kopfnomen. In (11b) und (11a) sind die Kopfnomen unmarkiert.

a. [Manan pika-l.ara-m] $]_{N P} \quad e-h a$.

1SG.ADE bull-ADJZ.husband-1SG be-AOR.3SG

'Ich hatte einen Stier-Mann (wrtl.: Mein Stier-Mann war.).'

(MNS_XX_BullSon_flk_132, Z: Narym)

b. [Manan waక̌ว $]_{N P} \bar{e}-t t a$.

1SG.ADE meat be-IPFV.3SG

'Ich habe Fleisch (wrtl.: Mein Fleisch ist.).'

(MNS_1984_BrotherSister_flk_40, Z: Narym)

c. A man as tunu-za-w, što [manan mat-kin] $]_{N P} q i b a$ jī-w but 1SG NEG know-PST-1SG.O, what 1SG.ADE house-LOC small son-1sg t'eli-mpa-n.

give.birth-PST.REP-3SG.S

'Ich wusste nicht, daß in meinem Haus mein kleiner Sohn geboren wurde.' (PMP_1966_BoyDevil_flk_21, S: Middle Ob)

${ }^{3}$ Die Formen ließen sich alternativ auch als Genitiv glossieren, man-a-n '1SG-EP-GEN'. In der vorliegenden Arbeit wird jedoch davon ausgegangen, dass es sich bei der Form um einen Adessiv handelt, da es auch Beispiele mit eindeutigem Adessiv gibt. 
d. [Manan aža-m] $]_{N P} \quad k u-m b a$. 1SG-ADE father-1SG die-PST.REP.3SG

'Mein Vater starb.' (SAI_1984_StoryAboutLifeLong_nar_4, Z: Narym)

e. [Man-nan miga-m] $]_{N P}$ al'd'a slle. 1SG-ADE needle-1sG fall.3SG down

'Meine Nadel fällt runter.' (TMR_1981_Robbers_flk_17, S: Middle Ob)

Desweiteren finden sich in beiden Dialektgruppen Beispiele, in denen man als Possessivpronomen mit dem Suffix -nanni (im Südselkupischen) bzw. -nanto (im Zentralselkupischen) verwendet wird. Nach Hajdú (1975) ist dieses Kasussuffix aus der Postposition -nān/i $/ 4$ entstanden, die im selkupischen Sprachgebrauch sowohl lokativische als auch ablativische Bedeutung hat(te) ${ }^{5}$. Das Suffix tritt (praktisch) auschließlich mit belebten Nomen und Pronomen auf und zeigt damit die einzige Umgebung, in der Animatheit im Selkupischen heute noch distinktiv ist ${ }^{6}$.

Eine zweite Interpretationsmöglichkeit ist die Betrachtung des Suffixes als Adessiv (ADE) mit einem zusätzlichen Adjektivierer -j bzw. in der Variante -i. In den Beispielen werden diese Fälle mit ADE/ABL glossiert. Pronominale Beispiele erscheinen ausschließlich in südlichen Ob- und Ket'-Dialekten, im zentralselkupischen NarymDialekt sind einzelne nominale Beispiele belegt. In Beispiel (12a) erscheint das Possessivpronomen man - '1SG' vor dem nominalen Kopf p'arg'ew - 'Bauch.mein'. Das Kopfnomen steht im Nominativ und ist mit dem Possessivsuffix der 1. Person markiert. In (12b) sind die Kasussuffixe mit den Possessivpronomen man - '1SG' und tan - '2sG' verschmolzen. Beispiel (12c) zeigt tan-nani - '2SG-ADE/ABL' als Possessvipronomen. Das Kopfnomen enthält das Genitivsuffix $-\boldsymbol{n}$ und die Variante -di des Possessivsuffixes der zweiten Person.

a. Kolobokka, Kolobokka, [man-nani p'arg'e-w] $]_{N P} \partial k$ Kolobokka, Kolobokka, 1sG-ADE/ABL stomach-1SG NEG.IMP poža-ľ̆e-šp-le-l. unpick-TR-IPFV1-OPT-2SG.O

'Kolobokka, Kolobokka, trenne nicht meinen Bauch auf.' (TFF_1967_KolobokkaPönegessa_flk_21, S: Middle Ob)

\footnotetext{
${ }^{4} \mathrm{Im}$ Nordselkupischen wird die Postposition noni noch verwendet.

${ }^{5}$ Hajdú geht sogar soweit, zu postulieren, dass sich die Funktionen von Lokativ und Ablativ in einigen Dialekten - wie den Ob-, Ket'- und Narym-Dialekten in den Beispielen - nicht voneinander unterscheiden (Hajdú 1975: 8)

${ }^{6}$ Unbelebte Nomina werden im Ablativ mit dem Suffix -qini markiert. Auch bzgl. des Lokativs lässt sich die Relevanz der Animatheit morphologisch beobachten: Belebte Nomina und Pronomina sind im Lokativ mit dem Suffix -nan markiert, das im Korpus als Adessiv ADE glossiert ist. Unbelebte Nomina dagegen mit dem Suffix qin bzw. qit. Im Südselkupischen gibt es sogar in narrativen und Folkloretexten keine unbelebten Nomina oder Pronomina mit -nan, im Zentralselkupischen finden sich einzelne Beispiele ebenso wie in südselkupischen Übersetzungen.
} 
b. Era-t t'ari- $\eta$ tāl as [tanani sü̈-di, tau [sü-di husband-3SG say-3SG.S this NEG 2SG.ADE/ABL sew-TR, this sew-DRV manani nan'a- $u-n]_{N P}$. 1SG.ADE/ABL sister-1SG-GEN

'Der Ehemann sagt: das ist nicht deine Näharbeit, das ist die Näharbeit meiner Schwester.'

(PMP_1961_ForestWoman_flk_199, S: Middle Ob)

c. Man tšă̌̌a- $\quad$ [tan-nani èra-ndi-ne $]_{N P \text {. }}$

1SG go-1SG.S 2SG-ADE/ABL husband-OBL.2SG-DAT/ALL

'Ich gehe zu deinem Mann.'

(PMP_1961_Fairytale_flk_151, S: Upper Ob)

Das Possessivpronomen der zweiten Person Singular wird ebenfalls in beiden Dialektgruppen verwendet. Analog zum Possessivpronomen der 1. Person erscheint das Pronomen aufgrund der Alternation $t \sim n$ in den Varianten tan und tat. Die Variante tan wird praktisch nur im Südselkupischen verwendet, die Variante tat hingegen erscheint vorrangig im Zentralselkupischen. Ebenso wie bei man/mat werden in beiden Dialektgruppen die Kopfnomen in Konstruktionen, die eine „inalienable possession“ ausdrücken, ebenfalls PX-markiert wie in (13b) - (13b), in Konstruktionen mit „alienable possession“" hingegen nicht wie in Beispiel (15) und (14c). Die Verteilung von markierten und unmarkierten Kopfnomen ist allerdings nicht so eindeutig wie im Falle von man/mat. In Tabelle 4 ist erkennbar, dass im Bereich der ,alienable possession“ weniger unmarkierte als PX-markierte Kopfnomen vorkommen, wobei die Gesamtanzahl der Beispiele mit 7 ohnehin nicht sehr aussagekräftig ist. Im Bereich der „,inalienable possession“ hingegen überwiegen PX-markierte Kopfnomen deutlich.

Tab. 4: Possessivpronomen 2. Person und PX-Markierung des Kopfnomens

\begin{tabular}{l|ll} 
& PX-markiert & unmarked \\
\hline „alienable“ & 5 & 2 \\
„inalienable“ & 52 & 5 \\
\hline
\end{tabular}

Beispiele finden sich vor allem mit dem Possessivpronomen im Nominativ, Adessivmarkierung wie in (14c) sind vereinzelt in den südlichen Dialekten belegt. Auch mit dem Possessivpronomen der 2. Person kommen Formen wie in (14b) vor, in denen das Pronomen mit dem Suffix -nanni markiert ist (für weitere Erläuterungen siehe unter 2.1.2.2). In Beispiel (15) erfolgt am Kopfnomen keine PX-Markierung, obwohl es aus dem Bereich der ,inalienable possession“ stammt.

a. A, [tan čapte $]_{N P}$ fae , kade-mba-t korg. INTERJ 2SG fairytale good say-PST.REP-3SG.O bear

'Ah, dein Märchen ist gut, sagte der Bär.'

(KIA_2014_SelkupBear_trans_27, Z: Narym) 
b. Im'a-l paja ităa-l-gu-k: Was'il'îsa-l] woman-ADJZ old.woman say-INCH-ITER-3SG.S 2SG Wasilisa-2SG

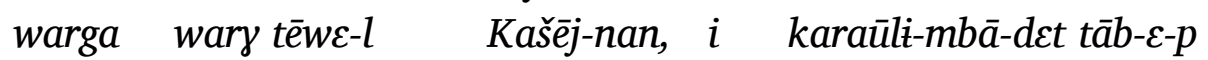
live.3SG big tooth-ADJZ Kashej-ADE and guard-HAB-3PL 3SG-EP-ACC koč qū-la.

much person-PL

'Die Zauberin sagte: Deine Vasil'isa lebt bei Kašej mit den großen Zähnen, und viele Leute bewachen sie.'

(TTD_1964_Frog_flk_108, Z: Tym)

a. Natän nagir-gin nagi-r-ba-n:

[tan eze-l] $]_{N P} \quad q u-\eta$ there letter-LOC write-FRQ-PST.REP-3SG.O 2SG father-2sG rich-ADV je-wa- $n$.

be-AOR-3SG.S

'Dort im Brief war geschrieben: Dein Vater ist reich.' (PMP_1961_Fairytale_flk_13, S: Upper Ob)

b. Ne-d'ey t'ari- $\eta$ : tau-n ugon qaj [ta-nani nan'a-l] $]_{N P}$ woman-DRV say-3SG.S DEM-GEN earlier what 2SG-ADE sister-2SG mēgga sü̈d-ə-r-ku-s. 1SG.DAT Sew-EP-FRQ-ITER-PST.3SG

'Die Frau sagt: bis jetzt, was hat deine Schwester mir genäht?' (PMP_1961_ForestWoman_flk_195, S: Upper Ob)

c. Ū.paja paja, quša-j [tan-nan sūrom] $]_{N P}$ oh.old.woman, old.woman, how.many-ADJZ 2SG-ADE wild.animal kōc' kōc' könde-lla, ì-l'e-nt mazim pir'a-yən-t, much much horse-PL, take-OPT-2sG.S 1SG.ACC oneself-LOC-3SG wark-la-j oqir-ma-yin. live-OPT-1DU one-ABST-LOC

'Oh Frau, Frau, wieviel Vieh du hast (wrtl.: wieviel dein Vieh ist), wieviele Pferde, nimm mich zu dir, lass uns zusammen leben.' (PMP_1966_BodylessHead_flk_87, S: Middle Ob)

(15) [Tat indiga- $\gamma]_{N P}$ mazik n'aja-ľ̌̆ $-\gamma a$.

2SG daughter-in-law-DIM 1SG.ACC anger-INT.PF-AOR.3SG

'Deine Schwiegertochter hat mich geärgert.'

(ChDN_1983_MistressOfFire_flk_62, Z: Vasjugan)

Das Possessivpronomen der dritten Person tab erscheint aufgrund der Alternation $b \sim p$ auch in der Variante tap. Es kann im Singular und Plural als Modifikator eines nominalen Kopfes auftreten. Das Possessivpronomen erscheint sowohlin den Zentralals auch in den Süddialekten vor allem im Genitiv wie in (16b) und (16d) und im Adessiv wie in (16a) und (16c) vor, wobei die Genitivmarkierung häufiger vertreten ist als die Adessivmarkierung. Beispiel (17) aus dem südselkupischen Middle Ob-Dialekt 
zeigt das einzige Beispiel im im Nominativ. In Beispiel (16a) ist im Personalpronomen noch die (alte) Genitivmarkierung ( $-n$ ) vor dem Lokativsuffix erkennbar. Das Pluralsuffix - $d$ im zentralselkupischen Beispiel (16b) ist ein Allomorph zum Pluralmarker -t. Die NP in (16c) ist neben dem Personalpronomen zusätzlich mit einem Adjektiv (warq - 'groß') modifiziert.

Das System der possessiven Markierung des Kopfnomens ist bei der 3. Person am wenigsten intakt. Tabelle 5 zeigt eine relativ eindeutige Zuordnung bei der ,inalienable possession“. Im Gegensatz dazu überwiegt auch bei der „alienable possession“ die

Tab. 5: Possessivpronomen 3. Person und PX-Markierung des Kopfnomens

\begin{tabular}{l|ll} 
& PX-markiert & unmarked \\
\hline „alienable“ & 12 & 19 \\
„inalienable“ & 67 & 50 \\
\hline
\end{tabular}

PX-Markierung wie schon beim Possessivpronomen der 2. Person. Ein Grund hierfür sind übersetzte Texte vor allem aus zentralen Dialekten, in denen häufig markierte Kopfnomen aus dem Bereich „alienable“ vorkommen, als auch unmarkierte aus dem Bereich „inalienable “. In den südlichen Dialekten ist die Zuordnung eindeutiger.

(16) a. [Tab-i-n-nan fa paja-d $]_{N P}$ šande ku-mba. 3SG-EP-GEN-ADE good woman-3SG young die-PST.REP.3SG

'Seine schöne Frau ist jung gestorben.'

(ChDN_1983_HeroesDaughter_flk_2, Z: Vasjugan)

b. [Tab-i-d-i-t el] $]_{N P}$ mad'e-t ku-tko ed'e-la-de.

3SG-EP-PL-EP-GEN soul taiga-GEN person-TRL become-OPT-PL

'Ihre Seelen werden zu Waldmenschen.'

(ChDN_1983_TaleBrownies_flk_4, Z: Vasjugan)

c. [Tab-la-nan warq paq-la-dit $]_{N P}$ tèli-mba-dit.

3SG-PL-ADE big knife-PL-3PL sparkle-PST.REP-3PL

'Ihre großen Messer glitzerten.'

(TMR_1981_Robbers_flk_19, S: Middle Ob)

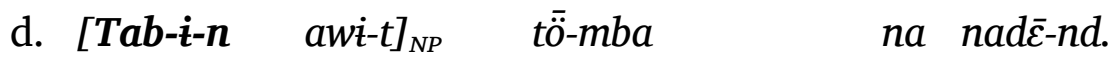

3SG-EP-GEN mother-3SG come-PST.REP.3SG this girl-ILL

'Ihre Mutter kam zu diesem Mädchen.'

(TMR_1981_Devil_flk_25, S: Middle Ob)

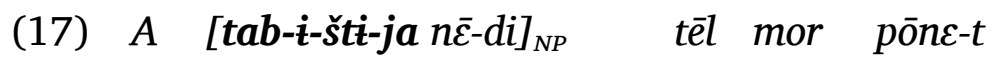

but 3SG-EP-DU daughter-DU day whole outward(s)-LOC.ADV

kur-t-pa-q.

rUn-IPFV-PST.REP-3DU.S

'Aber ihre Töchter liefen den ganzen Tag auf der Straße.'

(TMR_1981_Robbers_flk_6, S: Middle Ob) 


\subsubsection{Interrogativpronomen}

Das Selkupische unterscheidet eine Reihe von Interrogativpronomen, deren Varianten in beiden Dialektgruppen in Tabelle 6 dargestellt sind. Die Interrogativpronomen kak - 'wie', katora - 'welche' und qāqi - 'welche' sind aus dem Russischen entlehnt. Interrogativa können ähnlich wie Nomen dekliniert werden, wie z.B. kutim - 'wer.ACC' oder qajim - 'was.ACC'. Die im Nordselkupischen vorkommende emphatische Variante kutika - 'wer.EMPH' lässt sich im Selkup Language Corpus nicht belegen. Interrogativpronomen erscheinen typischerweise in Interrogativsätzen bzw. einige auch als Relativpronomen. Das Interrogativpronomen qaj - 'was' tritt als Modifikator auch

Tab. 6: Interrogativpronomen

\begin{tabular}{|c|c|c|}
\hline Zentralselkupisch & Südselkupisch & \\
\hline kudi, kuti, kude, kud, kod & kuti, kutti, kut, kot, kod & 'wer' \\
\hline qaj, kaj, qai, kai & qaj, kaj, qai, kai & 'was' \\
\hline $\begin{array}{l}\text { kak, kanduk, qandar, qutar, qa- } \\
\text { jinduk, qāja }\end{array}$ & kak, qaqä, kundar, kuddar & 'wie' \\
\hline$k u, k \bar{u}, k u c ̌ a, k u n$ & $\begin{array}{l}\text { ku, kuča, kuača, kuša, kunni, ku- } \\
\text { t'e, kut'ed'el'e }\end{array}$ & 'wo' \\
\hline kušša, kušša & kussa, kusa, kuša, kušša & 'wieviel' \\
\hline kuza & kuza, guža, qayə & 'wann' \\
\hline katora, yandul & qul'd'i, kuld'i, qāqi & 'welche' \\
\hline
\end{tabular}

in der Bedeutung von 'welche Art von' auf, wie die Beispiele (18a) und (18b) zeigen. Der Kopf der NP struška-la - 'Hobelspan-PL' ist eine Entlehnung vom Russischen struzka - 'Hobelspan'. Beispiel (18c) zeigt das Interrogativpronomen kuša - 'wieviele' als Modifikator der NP sūrom - 'wildes Tier', Das Interrogativpronomen ist mit dem Adjektivmarker -j abgeleitet. In Beispiel (18d) ist das Interrogativpronomen kuša mit dem Adjektivmarker $-j$ abgeleitet und erscheint mit der Bedeutung 'einige'.

(18) a. Ataman t'ari-n: tau [qaj struška-la] $]_{N P} \check{c} \bar{a} z \partial-t a-t t \partial ?$ Ataman say-3sG.S: there what shaving-PL run-IPFV2-3PL

'Der Ataman sagt: Welche Art Holzspäne schwimmen dort?' (PVD_1961_FarmAssault_flk_29, S: Upper Ob)

b. Na paja-ga ż̌̆a-l-gwa: na [qaj ì] $]_{N P}$, na pēge. this woman-DIM say-INCH-ITER.3sG: this what son, this hazel-grouse 'Diese Alte sagte: Welche Art Sohn ist das, das ist ein Haselhuhn.' (SEV_1980_HazelGrouse_flk_12, S: Middle Ob)

c. ... [kōó' kōo' könd-ə-lla $]_{N P}$, ì-l'e-nt mazim pir'a-yənt, much much horse-EP-PL, take-OPT-2sG.S 1SG.ACC himself-LAT-3SG, 
wark-laj oqir-majin.

live-IMP.1DU one-near

'... viele, viele Pferde, nimm mich zu dir, lass uns zu zweit leben!'

(PMP_1966_BodylessHead_flk_87, S: Upper Ob)

d. Tab-i-p war-za-t $[k u s ̌ a-j \quad p o-t]_{N P}$.

3SG-EP-ACC keep-PST-3PL how.many-ADJZ year-LOC.ADV

'Sie behielten ihn einige Jahre.'

(SEV_1980_HazelGrouse_flk_13, S: Middle Ob)

\subsubsection{Indefinitpronomen}

Indefinitpronomen werden von Interrogativpronomen mit Hilfe von Partikeln und Klitika gebildet. Während in nordselkupischen Dialekten 3 Partikeln verwendet werden (ēmä, qam/qap, koš/qos/kos), verwenden die zentralen und südlichen Dialekte vorrangig die aus dem Russischen entlehnte Partikel -to zur Bildung von Indefinitpronomen. Im Korpus erscheint die Form meist als -ta oder - da. Tabelle 7 zeigt die mit Korpusdaten belegten Indefinitpronomen in beiden Dialektgruppen.

Als Attribut einer Nominalphrase erscheinen Indefinitpronomen stets in pränomina-

Tab. 7: Indefinitpronomen

\begin{tabular}{|c|c|c|}
\hline Zentralselkupisch & Südselkupisch & \\
\hline qajto & qajda & 'irgendwas' \\
\hline kuč'atta, kuda & kunda & 'irgendwo’ \\
\hline$q u d \partial d \bar{a}$ & kudta & 'irgendwer' \\
\hline kučata & & 'irgendwann' \\
\hline qandukta & & 'irgendwie' \\
\hline & kuttidaka & 'irgendwer' \\
\hline$k u d \dot{t}-k o j$ & & 'irgendwo' \\
\hline kun'ibet & & 'irgendwohin' \\
\hline kujem & kujem & 'irgendwohin' \\
\hline & qajmemma, qajami & 'irgendwas' \\
\hline kužānem & kut'änemmi & 'irgendwann' \\
\hline & kundaremma & 'irgendwie' \\
\hline qajl'emìl & & 'irgendwelche' \\
\hline
\end{tabular}

ler Stellung und werden mit Hilfe des Suffixes -ta - 'INDEF' vom Interrogativpronomen qaj - 'was' gebjldet. Das Suffix tritt hier in der Variante -da auf.

Die Beispiele (19a) und (19b) zeigen Indefinitpronomen als Attribute einer Nominalphrase. Sie erscheinen stets in pränominaler Stellung. In südselkupischen Ket'- 
Dialekten und im zentralselkupischen Narym-Dialekt wird auch die Partikel ämmi verwendet. In beiden Beispielen (19e) und (19f) ist die Form verkürzt zu me, in Beispiel (19f) tritt sie in der Variante jem auf.

a. [Kai-la-da hir $]_{N P}$ nate-n nin-ga. what-DRV-INDEF cow there-LOC.ADV stand-AOR.3SG

'Irgendeine Kuh steht dort.' (ChDN_1983_BearCameIntoVillage_flk_5, Z: Vasjugan)

b. [Kaj-da ara-ľ̆i-ga-p $]_{N P} \quad \check{c} w \varepsilon d \varepsilon-m b a-t$. what-INDEF old.man-DIM-DIM-ACC meet-PST.REP-3SG.O

'Sie traf irgendeinen alten Mann.' (MNS_XX_BullSon_flk_105, Z: Narym)

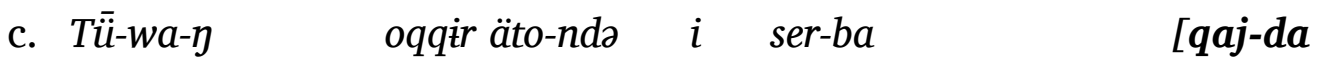
come-AOR-3SG.S one village-ILL and come_in-PST.REP.3SG what-INDEF moqo-qa māt-to] $]_{N P}$. back-LOC house-ILL

'(Er) kam in ein Dorf und ging in irgendein hinteres Haus.' (AGS_1968_FairytaleSnake_flk_84, S: Middle Ket')

d. Nenn'a-t [qaj-da tebi-l-zu-n-d] $]_{N P}$ sister-3SG what-INDEF man-ADJZ.person-DAT/ALL-3SG ว̄̌̆a-l-gwa. say-INCH-ITER.3SG

'Die Schwester sagte zu irgendeinem Mann.' (SEV_1981_SisterBrother_flk_73, S: Middle Ob)

e. Pe-t medì-la, nāda šeqqu-gu, pō-p night-LOC.ADV achieve-CVB, necessary spend.night-INF, tree-ACC paž'a-l-gu, tü̈-p č'adi-gu, [qal'-me-l kurpa-p] $]_{N P}$ chop-INCH-INF, fire-ACC burn-INF, what-INDEF-ADJZ cereals-textscacc pōt-ku, č̄āj pöt-če-gu. cook-INF, tea cook-INF

'Wenn die Nacht kommt, muss man übernachten, Holz hacken, Feuer anzünden, irgendeine Grütze kochen, Tee warm machen.'

(SAA_1984_MyGrandmother_nar_5, Z: Narym)

f. Qwat-tša-l' [qaj-jem pak'k'e-m] $]_{N P}, \ldots$ kill-TR-2SG.O what-INDEF teal-ACC

'Wenn du irgendeine Krickente tötest, ...' (MMP_1964_Lgov_trans_17, S: Middle Ket')

${ }^{7}$ Die Partikel ist ursprünglich die 3. Person Singular Konjunktiv des Verbs sein (vgl. Prokof'ev 1935: 44), A. I. Kuznecova/Chelimskij/Grushkina 1980: 299, Bekker 1995b: 126 


\subsubsection{Demonstrativpronomen}

Im Zentral- und Südselkupischen wird zwischen proximalen und distalen Demonstrativpronomen unterschieden. $\mathrm{Zu}$ den proximalen Demonstrativpronomen gehören na, aw, naw, taw, tau und tam 'dieser', zu den distalen gehören to, taj und tina 'jener'. Darüber hinaus existiert nil'‘̌̌i, nidi, ni 'solcher' zur Angabe der Beschaffenheit. Das aus dem Russischen entlehnte takoj 'solch' gehört ebenfalls hierher. Die Pronomina na und tina werden ausschließlich anaphorisch verwendet. Die Demonstrativpronomen erscheinen ausnahmslos pränominal. Es gibt keine Kongruenz zum Bezugsnomen.

\section{proximal}

Im Beispiel (20) ist der Kopf der NP kibajče - 'Junge' mit dem proximalen Demonstrativum na - 'dieser' modifiziert. Das Adjektiv kiba - 'klein' ist Modifikator der NP andoyindi - 'Boot.ABL'.

(20) [Na kibajč $]_{N P} k i b-a n d o-\gamma \dot{i n d i}$ čanni-mba, kib.andi-p DEM boy small.boat-ABL get.off-PST.REP.3SG, small.boat-ACC

$q \bar{\partial}-t \quad k \bar{r} r a-n d$ natidēli čāččit $t, \quad$ onžŏ coast-GEN sand-ILL there pull.out-3SG.O, himself into.forest qwan-ba. go.away-PST.REP.3SG

'Dieser Junge stieg aus dem Boot aus, zog das kleine Boot auf den Ufersand, er selbst ging in die Taiga.'

(SEV_1981_SisterBrother_flk_22, S: Middle Ob)

Die proximalen Modifikatoren in (21a), (21b) und (21c) zeigen die phonetischen Variationsmöglichkeiten der selkupischen Dialekte, zum Teil bei ein und demselben Sprecher. Der nominale Kopf in (21a) und (21b) ist ein Kompositum und setzt sich aus dem denominalen Adjektiv näj - 'weiblich' und dem Nomen qum - 'Mensch' zusammen.
a. $[\text { Tau nä-j-qum] }]_{N P} \quad$ tü-a-n tab-i-n $n \quad i-m-t$ DEM woman-ADJZ-person come-AOR-3SG.S 3SG-EP-GEN son-ACC-3SG tau-gu.
buy-INF
'Diese Frau kommt, um ihren Sohn zu kaufen.'
(PMP_1961_Fairytale_flk_38, S: Upper Ob)
b. [Taw nä-j-qum $]_{N P} \quad$ okkipnadi mādo-r-ni- $\eta$ : DEM woman-ADJZ-person alone ask.for-FRQ-AOR-3SG.S mer-i-nt $i$ mer-i-nt. price-EP-2sG.S and price-EP-2sG.S 'Diese Frau bittet allein: Verkauf ihn und verkauf ihn!' (PMP_1961_Fairytale_flk_41, S: Upper Ob)
c. Qaj-yən je- $\eta \quad$ [aw siga-laga-l $]_{N P}$. what-LOC be-3SG.S DEM ball-SING-2SG


'Wo ist dein anderes Knäuel?'

(PMP_1966_BodylessHead_flk_13, S: Upper Ob)

\section{distal}

Als distale Demonstrativpronomen sind im Textkorpus die selkupischen Varianten taj, to und nidi - 'jener' sowie die aus dem Russischen entlehnten Varianten takoj und tut - 'jener' belegt. Beispiel (22a) zeigt das distale Demonstrativpronomen taj - 'jener' als Modifikator der NP. In Beispiel (22b) erscheint das Demonstrativum to - 'jener' vor dem Nominalen Kopf bleka-nd - 'Seite-ILL'. Der zweite Modifikator der NP tü 'Feuer' ist eigentlich ein Genitivkomplement wie in Beispiel (23b). Allerdings steht er hier in der unmarkierten Form. Beispiel (22c) zeigt das Demonstrativpronomen nidi'solcher' als Modifikator der NP. Am nominalen Kopf $q u-n$ - 'Mensch-PL' ist der Plural markiert.

a. ...pü-ga-k [taj mosto-win $]_{N P}$.

cross-ITER-IMP.2SG.S DEM bridge-PROL

'... überquere jene Brücke!'

(KMS_1966_MouseGray_flk_20, S: Middle Ket')

b. Mat mid-o-m [tü to bleka-nd] $]_{N P}$ onenže

1SG liver-EP-1SG.NOM/ACC fire DEM side-ILL yourself

pire-yend pen-de.

stature-ILL.3SG put-IMP.2SG.O

'Leg meine Leber auf jene Seite des Feuers, gegenüber von dir.' (ChDN_1983_GirlAndIce_flk_29, Z. Vasjugan)

c. [Nidi qu-n] $]_{N P} \quad$ e-ja-dit, $\quad$ tab-i-n mat-ti wargi-dit, m'i such person-PL be-AOR-3PL, 3SG-EP-PL house-ILL live-3PL, 1PL tab-d-i-p aya konze-r-na-ut. 3SG-PL-EP-ACC NEG See-FRQ-AOR-1PL

'Es gibt solche Menschen, sie leben im Haus, wir sehen sie nicht.' (ChDN_1983_TaleBrownies_flk_1, Z: Vasjugan)

Das Demonstrativpronomen takoj - 'solcher' in Beispiel (23a) ist eine russische Entlehnung, ebenso wie der nominale Kopf dieser NP l'en' - 'Faulenzer'. Auch in Beispiel (23b) ist das Demonstrativum eine Entlehnung aus dem Russischen, in diesem Fall von russisch tot - 'jener'. Der Modifikator der NP iga imde - 'Sohn ihres Sohnes' erscheint im Akkusativ.

a. İ-de qai-m-n.as m'e-ku-t, [takoj l'en'] ${ }_{N P}$ son-3SG what-ACC-EMPH.NEG do-ITER-3SG.O such sluggard t'eli-m-a-t.

sun-TRL-EP-3SG.O

'Ihr Sohn hat nichts getan, ein solcher Faulenzer ist geboren.' (KMS_1963_Sluggard_flk_4, S: Middle Ket') 
b. [Tut kit-t par-yit $]_{N P}$ paja-l'ži-ka warga i-ga that river-GEN top-LOC old.woman-DIM-DIM live.3SG son-DIM $i-m-d e$.

son-ACC-3SG

'Am Ufer (wrtl.: am oberen Ende) jenes Flusses lebt eine alte Frau (und) ihr Enkel (wrtl.: Sohn ihres Sohnes).'

(ChDN_1983_Nikita_flk_1, Z: Vasjugan)

\subsubsection{Emphatisches Pronomen}

Das emphatische Pronomen im Selkupischen basiert auf dem Stamm on- und flektiert nach Numerus (Singular, Dual, Plural) und Person seines Bezugsnomens. Helimski (1998: 563) bezeichnet es als emphatisch-reflexives Pronomen mit einem gegenüber den Nomen reduzierten Kasusparadigma (die Lokalkasus bis auf Dativ/Allativ fehlen). Im Gegensatz dazu gibt Bekker (1995b: 86ff.) ein vollständiges Kasusparadigma an und beschreibt das Pronomen als reflexives mit unterschiedlichen Bedeutungen (als attributives Pronomen wie russ. sam - 'selbst', als reflexiv-possessives Pronomen wiw russ. svoj - 'eigener' und als reflexives Pronomen wie russ. sebja - 'sich'), in Abhängigkeit von morphologischen und syntaktischen Faktoren. Über den Umfang des Kasusparadigmas lassen sich keine Aussagen treffen, da die Kasussuffixe an den Beispielen aus dem Textkorpus oftmals nicht mehr eindeutig identifiziert werden können. Es finden sich aber Beispiele, in denen volle Kongruenz zwischen dem Pronomen und dem nominalen Kopf bestehen, wie in (25a) mit Genitiv-Markierung und (25b) mit Illativ-Markierung.

Als Modifikator einer Nominalphrase erscheint das emphatische Pronomen in der Bedeutung 'eigener'. Beispiele finden sich für die erste, zweite und dritte Person Singular, wie in (24a) - (24c), die erste Person Dual, in Beispiel (24d), sowie die zweite und dritte Person Plural, wie in (24e) und (24f). Das Pronomen erscheint konsequent pränominal, in Beispiel (24e) tragen sowohl das Pronomen als auch das Kopfnomen ein Possessivsuffix.

a. [Onek nena-m] $]_{N P} \quad m e-l a-k-s$.

myself sister-ACC.1SG give-OPT.1SG.S-CLIT

'Meine eigene Schwester gebe ich (dir).'

(NN_1855_Hero1_song_348, Z/S: Tym/Middle Ob)

b. Ol-laga t'ari- $\eta$ : könt [onent mista-yənt] $]_{N P}$ qwan-qə, head-SING say-3SG.s horse yourself place-LOC.3SG go.away-IMP.2SG.S t'egi-l'-te-k! untie-INCH-DRV-IMP.2SG.S

'Der Kopf sagte: Pferd, geh auf deinen eigene Platz, spann dich aus!' (PMP_1966_BodylessHead_flk_44, S: Middle Ob)

c. Ǚ̌ed'e-l'i-ka ära-n tö-mba, na kwilì-p child-DIM-DIM old.man-DAT/ALL come-PST.REP.3SG this fish-ACC 
[onže ando-yonde $]_{N P}$ pen-ba-t. himself boat-ILL.3SG put-PST.REP-3SG.O

'Der Junge kam zu dem Alten und legte diesen Fisch in sein eigenes Boot.' (ChDN_1983_Nikita_flk_19, Z: Vasjugan)

d. Mì-nan [on solon-mut $]_{N P}$ koč' $\bar{e}$-ja.

1DU-ADE ourselves.DU gold-PROL-POSS.1PL much e-AOR.3SG

'Wir haben viel eigenes Gold.'

(SDP_1964_FairytaleBlackZar_trans_598, Z: Narym)

e. Könt-la [onti-lt qwaja-yəntì-lt] $]_{N P}$ čāza-lta!

horse-PL yourself-2PL yard-ILL.3SG-2PL go-IMP.2PL

'Pferde, geht in euren eigenen Hof!'

(PMP_1966_BodylessHead_flk_48, S: Middle Ob)

f. N'uza qanž-la qäli-d'at, [unti-t kopto-yanto-t] $]_{N P}$ grass sledge-PL unload-3PL himself-PL place-ILL.3SG-PL

qwan-na-t.

go.away-AOR-3PL

'Die Heuschlitten haben sich entladen, sind auf ihre eigenen Plätze gegangen.'

(PMP_1966_BodylessHead_flk_54, S: Middle Ob)

(25)

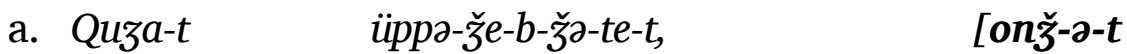

where-LOC.ADV set.off-RFL-HAB-IPFV-DRV-3SG.O himself-EP-GEN

tobə-t $]_{N P}$ wažว-m-də taqqa mažว-qu-t.

leg-GEN meat-ACC-3SG away cut.off-ITER-3SG.O

'Als er sich aufmachte, schnitt er ein Stück Fleisch von seinem eigenen Bein ab.'

(SDP_1964_FairytaleBlackZar_trans_135, Z: Narym)

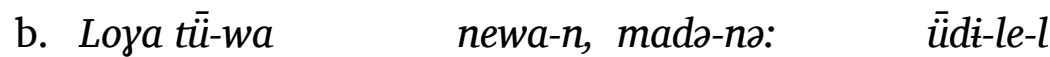

fox come-AOR.3SG hare-ILL ask.for-AOR.3SG let.go-INCH-2SG.O

māzip [onenž-i-ni mat-tว] $]_{N P}$ wargi-gu.

1SG.ACC yourself-EP-DAT/ALL house-ILL live-INF

'Der Fuchs kam zum Hasen und fragte: Lass mich zu dir, um in deinem Haus zu wohnen!'

(TET_1979_TheHaresHouse_flk_12, S: Middle Ket')

\subsubsection{Adjektive}

Die grundlegende Funktion von Adjektiven ist der Ausdruck des Attributs bei einem nominalen Satzglied. Attributive Adjektive im Selkupischen modifizieren also ihr Bezugsnomen. Daneben können sie als Prädikatsnomen wie in Beispiel (26a) auftreten. Prädikatsnomen sind in Kapitel 4 dargestellt. Wie andere Modifikatoren auch, treten attributive Adjektiven im Südselkupischen immer unmarkiert und pränominal auf. 
Ebenso wie im Enzischen ist keine Kongruenz zwischen einem Adjektiv und seinem Bezugsnomen belegt. Abgeleitete Adjektive sind mit dem Suffix -l bzw. der Variante -j markiert. Es können mehrere Adjektive innerhalb einer Nominalphrase gemeinsam vorkommen. Bezüglich einer möglichen Hierarchie kann aufgrund der wenigen Beispiele keine Aussage getroffen werden. In (26b) kommen drei abgeleitete Adjektive als Modifikatoren des Nominalen Kopfes gum 'Mensch' vor. Das Adjektiv tibbil 'männlich' formt mit dem Kopf das Kompositum 'Mann'. Ähnlich wie in (26b) sind auch die Adjektive in (26c) abgeleitet: tebel qut - 'männliche Menschen' und cumil nel qude 'selkupischer weiblicher Mensch'. Anders als im obigen Beispiel bilden die Adjektive kein Kompositum mit ihrem Kopfnomen. Neben abgeleiteten Adjektiven kommen auch nichtabgeleitete Adjektive vor, in Beispiel (26d) in zwei NPs. Der König wird dort als warq ol - 'großer Kopf' bezeichnet.

a. [tap nä-j-qum] $]_{N P} \quad \boldsymbol{s o ̄ t} k \boldsymbol{k}$.

this woman-ADJZ-person good

'Dies (ist) eine gute Frau.'

(PMP_1961_Fairytale_flk_141, S: Middle Ob)

b. [Siwa-j ors-u-j tibi-l-gum $]_{N P}$.

goodADJZ force-EP-ADJZ man-ADJZ.person

'Ein guter starker Mann (wrtl.: männlicher Mensch).'

(SEV_1980_Itja_flk_31, S: Middle Ob)

c. Mad'e-t [tebe-l qu-t $]_{N P 1}$ [čumi-l ne-l qu-de] $]_{N P 2}$

taiga-GEN man-ADJZ person-PL Selkup-ADJZ woman-ADJZ person-3sG

ella-dit.

live-3PL

'Die Waldmenschen leben mit selkupischen Frauen.'

(ChDN_1983_TaleBrownies_flk_13, Z: Vasjugan)

d. A pēge qu-t tarēdik čēnča: qöšk-le-nd [arg

but hazel.grouse person-GEN like say.3SG: go-OPT-2SG.S other

$\bar{\varepsilon} d \varepsilon-n d]_{N P 1}$, natt'at warka [warq ol] $]_{N P 2}$.

village-ILL, there live.3sG big head

'Aber das Haselhuhn sagt wie ein Mensch: du gehst in einen anderen Ort, dort lebt ein König (wrtl.: ein großer Kopf).'

(SEV_1980_HazelGrouse_flk_16, S: Middle Ob)

\subsubsection{Numeralia}

Als Modifikator innerhalb einer Nominalphrase kommen sowohl Kardinal- als auch Ordinalzahlen vor. Alle Numeralia sind unmarkiert und erscheinen stets pränominal. In der Subjektposition erscheint der nominale Kopf im Südselkupischen ausschließlich, im Zentralselkupischen und den Ketdialekten bis auf wenige Ausnahmen stets im Singular. Dieses Phänomen teilt das Selkupische nicht nur mit den samojedischen Sprachen, vielmehr ist es ein areales Charakteristikum (vgl. Fokos-Fuchs (1962: 
61ff.)). In der Objektposition erscheint der Kopf in den Zentraldialekten immer im Singular und einem obliquen Kasus, im Südselkupischen gibt es auch Beispiele, in denen der Kopf unmarkiert ist. Zwischen nominalem Kopf und Numeral gibt es keine Kongruenz.

\section{Kardinalia}

Im südselkupischen Beispiel (27a) sind zwei Nominalphrasen mit Numeralia modifiziert. Der nominale Kopf in NP1 qass - 'Schwimmer' steht in der Subjektposition, ist unmarkiert und wird neben dem Numeral noch mit einem unmarkierten Nomen poqqa - 'Netz' modifiziert. Der nominale Kopf in NP2 tut 'Karausche' steht in der Objektpositionen und ist ebenfalls unmarkiert. Das zentralselkupische Beispiel (27b) zeigt einen nominalen Kopf in Objektposition, markiert mit dem Akkusativ. Auch die Mehrzahl der südselkupischen Beispiele ist regulär mit dem Akkusativ markiert, wie in (27c). Ein Kopfnomen in Subjektposition kann in beiden Dialektgruppen mit einem Possessivsuffix markiert sein wie in (27d). Beispiele finden sich für alle vorhandenen Numeralia. Für die Objektposition finden sich hingegen keine entsprechenden Beispiele.

(27) a. [Nagūr poqqə qass] $]_{N P 1}$, [tätt tut $]_{N P 2}$ qwäti-mba. three net float, four crucian catch-EP-PST.REP.3SG

'Es sind drei Netzschwimmer, er fing vier Karauschen.' (TFF_1967_ItjaAldigaScale_flk_16, S: Middle Ob)

b. Mat [hel'd' pō-p] $]_{N P}$ paže-ka-p.

1SG seven tree-ACC chop-ITER-1SG.O

'Ich fällte sieben Bäume.'

(ChDN_1983_MistressOfFire_flk_87, Z: Vasjugan)

c. [Nāre tizi-m] $]_{N P} \bar{i}-a-t \quad$ wad'ä-m ninga sōya-nni-t.

three cup-ACC take-EP-3sG.O meat-ACC so put-AOR-3SG.O

'Sie nahm drei Schüsseln und füllte Fleisch hinein.'

(KMS_1966_TwoSisters_flk_61, S: Middle Ket')

d. Tab-i-štja-n-nan [nāyor ji-di] $]_{N P}$ èhayi, qwēčdi i fa 3SG-EP-DU-GEN-ADE three son-3DU be-AOR-3DU.s, beautiful and good i-la-di.

Son-PL-3DU

'Sie hatten drei Söhne, hübsche und schöne Söhne.' (TTD_1964_Frog_flk_2, Z: Tym)

\section{Ordinalia}

Ordinalzahlen werden mit Hilfe des Suffixes -mžel aus den Kardinalzahlen gebildet. Die überwiegende Mehrheit der südselkupischen Beispiele verwendet die Zahl zwei wie in (28a). Auch hier gibt es keine Kongruenz zum nominalen Kopf. Im zentralselkupischen Beispiel (28a) ist das pronominale Subjekt kovert und nur anhand der Verbalendung identifizierbar. Der nominale Kopf $t \bar{e} l$ - 'Tag' ist mit einer Ordinalzahl modifiziert. Analoge Beispiele finden sich auch im Südselkupischen. Das Beispiel (28b) 
aus dem Ket'-Dialekt enthält drei Nominalphrasen, die mit einer Ordinalzahl modifiziert sind. NP1 und NP2 sind elliptisch ausgelassen (siehe auch Abschnitt 2.1.2.13 auf Seite 44), der nominale Kopf in NP3 ist mit dem Akkusativ markiert und neben dem Numeral noch mit einem unmarkierten Nomen modifiziert. Der nominale Kopf kann zusätzlich zum Kasus mit dem Possessivsuffix der dritten Person markiert sein, wie in (28c).

a. [Šcdi-m̌̌šli tēl] $]_{N P}$ qöška poqq-la-m-d manni-mbi-gu.
two-ORD day go.3SG net-PL-ACC-3SG see-PST.REP-INF

'Am zweiten Tag geht er nach den Netzen schauen.' (TMR_1981_AboutItja_flk_7, S: Middle Ob)

b. Pēk'k'e laq-š-i-t qazä n'üži-m, [šədə-mdälži $]_{N P 1}$, hazel.grouse snap-US-EP-3SG.o bark grass-ACC two-ORD [nāri-mdälži $]_{N P 2}$, [tätti-mdeľ̌i qazä n'ǚ̆i-m] $]_{N P 3}$. three-ORD four-ORD bark grass-ACC

'Das Haselhuhn riß ein Riedgras, ein zweites, ein drittes, ein viertes Riedgras.'

(KMS_1963_HazelgrouseMouse_flk_30, S: Middle Ket')

c. [Šcdi-mžčli tēl-t $]_{N P 1} \quad$ tab aze-ind $\bar{\partial} \check{z} a-l-g w a:$

two-ORD day-LOC.ADV 3SG father-DAT.3SG Say-INCH-ITER.3SG

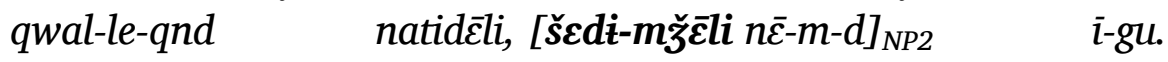

go.away-OPT-2SG.S there two-ORD daughter-ACC-3SG take-INF

'Am zweiten Tag sagte er zu seinem Vater: Du gehst dorthin, um die zweite Tochter zu nehmen.'

(SEV_1980_HazelGrouse_flk_36, Middle Ob)

\subsubsection{Quantifizierer}

In südselkupischen Texten erscheinen zwei verschiedene Quantifizierer: das selkupische koči - 'viele' und, in viel größerer Anzahl, das aus dem Russischen entlehnte wes' - 'alle'. Als Modifikator innerhalb einer Nominalphrase treten beide Quantifizierer analog auf: Sie stehen vor dem nominalen Kopf. Darüber hinaus kann koči auch als Adverb 'viel' erscheinen, dann mit dem Suffix - $k$. In den Beispielen aus dem Zentralselkupischen erscheint das Kopfnomen in Subjektpositionen immer im Nominativ Plural, in Objektposition im Akkusativ Singular. Die Beispiele aus dem Südselkupischen bieten kein einheitliches Bild. In Subjektposition kann der nominale Kopf im Nominativ Plural erscheinen, aber auch im Nominativ Singular. In der Objektposition finden sich Beispiele sowohl ihm Akkusativ Singular als auch im Akkusativ Plural. In den Beispielen (29a) und (29b) stehen die Kopfnomen in Subjektposition im Nominativ Singular. Der nominale Kopf kann in der Subjektposition auch im Nominativ Singular erscheinen, wie in (29c). Allerdings ist das Beispiel das einzige seiner Art. In den Beispielen erscheint der nominale Kopf in Objektposition immer mit dem Akkusativ markiert, wie in (29c). Im Südselkupischen findet sich ein Beispiel, in dem 


\section{Phrasentypen}

der Kopf im Plural statt im Singular erscheint (29c). Beispiel (29f) zeigt den aus dem Russischen entlehnten Quantifizierer wes'. Der nominale Kopf ist mit einem lokalen Adverbialkasus markiert.

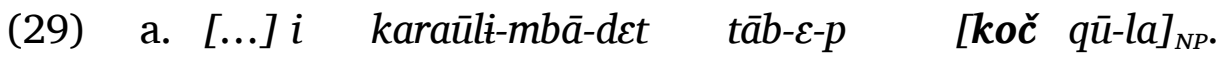
and guard-PST.REP-3PL 3SG-EP-ACC much person-PL

'[...] und viele Menschen bewachen sie.' (TTD_1964_Frog_flk_108, Z: Tym)

b. Manni-mpa-n $\quad$ ēe-j-qum: [koc' künt-la $]_{N P} \check{c} a \bar{z} \partial-n t a-t$ see-PST.REP-3SG.S woman-ADJZ-person much horse-PL run-INFER-3PL n'üzว kanžว-la-z'e. grass sledge-PL-INSTR

'Die Frau schaut: Viele Pferde laufen mit dem Heuschlitten.' (PMP_1966_BodylessHead_flk_28, S: Middle Ob)

c. Nil'd'z'i- $\eta$ waryi- $\eta$ t'ali-mpa-n, pel-galik, kak s'o rawno So-ADV big-ADV talk-PST.REP-3SG.S friend-CAR how it.doesn't.matter [koc'e qum] $]_{N P}$ t'äli-m-i-n-t.

much person talk-DRV-EP-AOR-3PL

'Er sprach so laut allein, als ob viele Menschen sprechen.' (PMP_1966_BodylessHead_flk_27, S: Middle Ob)

d. Tab i-mba-d

$[k \bar{o} \bar{c} \quad \check{s} \bar{w} w-\varepsilon-p]_{N P} \quad$ tü-nd, Pönege-t

3SG take-PST.REP-3SG.O much ashes-EP-ACC fire-ILL Pönege-GEN haj-o-nd čāčì-mba-d. eye-EP-ILL throw-PST.REP-3SG.O

'Er nimmt viel Asche aus dem Feuer, wirft sie in Pöneges Augen.' (TTD_1964_IdzhaKadzha_flk_8, Z: Tym)

e. İ-t sura-l'l'e qwan-ni- $\eta$, a ōnt son-3SG hunt-CVB go.away-AOR-3sG.s but himself qwa-ži-t ōnt paja-nti-ne qwädi-t go.away-IPFV3-3SG.o himself woman-OBL.3SG-DAT/ALL leave-3SG.O [kot' süra-n tar-la-m] $]_{N P}$. much wild.animal-GEN hair-PL-ACC

'Der Sohn geht jagen, aber er selbst lässt seiner eigenen Frau viele Felle.' (PMP_1961_ForestWoman_flk_164, S: Middle Ob)

f. Qari-maqin paja utä-l-qa-nni-t [wes morning-near old.woman let.go-INCH-ITER-AOR-3SG.O all kündi-la-m-dì $]_{N P}$ pōnä. horse-PL-ACC-3SG outward(s) 'Am Morgen lässt die Alte alle Pferde hinaus.' (AGS_1968_FairytaleSnake_flk_65, S: Middle Ket') 


\subsubsection{Partizipien}

Partizipien können als Modifikatoren in Nominalphrasen verwendet werden. Die Anzahl der Partizipien unterscheidet sich in den selkupischen Dialektgruppen. Für die nördlichen Dialekte werden die fünf Partizpien Präsens, Präteritum, Karitiv, Debitiv, Destinativ beschrieben (vgl. Helimski (1998), Cheremisina/Martynova (1991)8). Für das Südselkupische postuliert Cheremisina/Martynova (1991: 44ff.) das Vorhandensein der ersten drei Partizipien, in den Korpusbeispielen lassen sich jedoch (mit zwei Ausnahmen) lediglich Partizip-Präsens und Partizip-Präteritum belegen. N. G. Kuznecova (1995: 202ff.) 9 beschreibt ebenfalls drei Partizipien, auf -(m)pə(n)taj, -(n)tjaj und -pso bzw. -(p)såtaj, ohne den Adjektivmarker -l. Das erste drückt ihrer Meinung nach Präsens und Präteritum aus, das zweite wird zum Ausdruck von Perfekt und Plusquamperfekt verwendet, das letzte ist ein (selten vorkommendes) Partizip Destinativ. Im Textkorpus lassen sich im südselkupischen Ket'-Dialekt zwei Fall mit Partizip Karitiv nachweisen (Beispiele (31a) und (31b) unten), Partizip Destinativ hingegen weder in den zentralen noch in den südlichen Dialekten.

Hingegen ist das für das Nordselkupische beschriebene Partizip Debitiv 10 in der gemischten Dialektgruppe sowie den_üidlichen Dialekten Middle Ob, Chaja und Middle Ket' belegt (Beispiele (32a) - (32c)). Zentrale und nördliche Belege finden sich im Korpus nicht. Anders als Partizipien in nordsamojedischen Sprachen gehen selkupische Partizipien keine Kongruenz (hinsichtlich Kasus und Numerus) mit ihrem Bezugsnomen ein. Sie drücken aktive Bedeutungen aus. Die von Cheremisina/Martynova (1991) angenommene Möglichkeit, mit Partizipien passivische Bedeutung ausdrücken zu lassen, lässt sich im Textkorpus nicht nachweisen 11 .

Die Partizipialsuffixe weisen formale Unterschiede in den Dialekten auf. Im Zentralselkupischen wird im Partizip Präsens das Suffix -tìl, im Partizip Präteritum das Suffix -pill(e) verwendet, der Adjektivmarker - $l$ ist dort noch sichtbar. In den südselkupischen Texten wird dagegen im Partizip Präsens - ndi, ohne den Adjektivmarker, im Partizip Präteritum Formen mit Adjektivmarker (-mbile) und ohne (-mbidi, -lbidi) verwendet. Die Suffixe in den zentralen Dialekten und die Partizip Präsens Suffixe aus den südlichen Dialekten stimmen mit den von Cheremisina/Martynova (1991) beschriebenen überein. Die Marker im Partizip Präteritum der südlichen Dialekte passen zu den von N. G. Kuznecova (1995) beschriebenen. Anders als im Nordselkupischen tritt das Partizip im Zentral- und Südselkupischen, wie alle anderen Modifikatoren auch, immer pränominal auf (siehe A. I. Kuznecova/Chelimskij/Grushkina (1980: 339)). Die eindeutige Zuordnung von Partizipien zu Tempus lässt sich im Textkorpus nicht an-

\footnotetext{
${ }^{8}$ Cheremisina beschreibt südliche selkupische Dialekte „v severe Tomskoj oblasti [...]“, und bewegt sich damit eher im zentralselkupischen Raum, soweit es die Perspektive dieser Arbeit betrifft. Dies erklärt auch die partizipialen Formen auf -1, die im Textkorpus nur in zentralen Dialekten erscheinen.

${ }^{9}$ Kuznecova beschreibt mit dem Terminus Südselkupisch Dialekte an den Flüssen Tym, Narym und Ob (N. G. Kuznecova 1995).

${ }^{10}$ In der Arbeit wird die Glosse PTCP.NEC - 'participle necessiative' verwendet.

${ }^{11}$ Cheremisina/Martynova (1991) postuliert hier Ähnlichkeiten zum Chantischen, wo praktisch alle semantischen Rollen syntaktisch passiviert werden können.
} 


\section{Phrasentypen}

hand von Beispielen belegen. Partizip-Präsens-Beispiele können sowohl mit Verben im Präsens als auch im Präteritum stehen (vgl. Beispiele unten), das Gleiche gilt für das Partizip Präteritum.

Entsprechend der Akzessibilitätshierarchie nach Keenan/Comrie (1977: 66): Subjekt [SU]_Direktes Objekt [DO]_Indirektes Objekt [IO]_Oblique NP [OBL]_Genitiv-/ Possessor-NP [GEN]_Vergleichsobjekt [OCOMP] können im Zentral- und Südselkupischen unterschiedliche Komponenten relativiert werden, die in den folgenden Beispielen illustriert werden. In (30a) wird das Subjekt des Satzes kup - 'Mensch' mit dem Partizip Präsens modifiziert, die nachfolgenden Verbformen erscheinen im Präteritum. Die Beispiele (30b) und (30c) zeigen relativierte Objekte (direkt und indirekt), in Beispiel (30b) Partizip Präteritum mit Verbformen im Aorist 12 und Präteritum, in Beispiel (30c) Partizip Präsens mit der Verbform im Präteritum. Beispiel (30d) enthält eine oblique NP im Ablativ, die mittels des Partizips relativiert wird. In Beispiel (30e) wird die Genitiv-NP kudit - 'der Menschen' mit dem Partizip kubil - 'gestorbene' relativiert.

a. [Na mene-r-til kup $]_{N P}$ soho-tko $\varepsilon d e-m b a$, this hunt-FRQ-PTCP.PRS person wader-TRL become-PST.REP.3SG wašc-de-mba. fly-IPFV2-PST.REP.3SG

'Dieser Jäger (wrtl.: jagender Mensch) verwandelte sich in einen Watvogel und flog weg.'

(ChDN_1983_HeroesDaughter_flk_47, Z: Vasjugan)

b. Čawesse wes' tār-ni-di, čāmže [onž košti-l

backward all change-AOR-3DU.o frog himself bad-ADJZ

pana-l-bil poryi-p] $]_{N P} \quad \check{s e} r$-ba-t.

breal-INCH-PTCP.PST clothing-ACC dress-PST.REP-3SG.O

'Sie verwandelten sie zurück, der Frosch zog seine eigene alte, zerrissene Kleidung an.'

(MNS_1984_BrotherSister_flk_48, Z: Narym)

c. Medi- $\eta \quad$ [sü-n lejdi-mbidi qū-ne $]_{N P}$.

achieve-3SG.S dragon-GEN hire-PTCP.PST person-DAT/ALL

'Er kommt zum Drachenwächter.'

(PMP_1961_Fairytale_flk_128, S: Middle Ob)

d. $\quad \ldots a$ [t'üì-mbìdi qū-nanni $]_{N P}$ t'äyu-t tändì-nan

but come-PTCP.PST person-ABL NEG.EX-3SG.O 2SG.LOC which = EMPH

$k u l ' d i=n a \quad$ ärí-mbí-tä.

save-HAB-NMLZ

'Aber vor dem Menschen, der kommt, gibt es für dich keine Rettung.' (KMS_1963_UnknownMan_flk_10, S: Middle Ket')

\footnotetext{
${ }^{12}$ Das perfektive Verb tār- drückt mit dem Aorist die unmittelbare Vergangenheit aus.
} 
e. Na aja qu-t, na [ku-bil $k u-d-\dot{t}-t \quad$ elle-dit $]_{N P}$ this NEG person-PL this die-PTCP.PST person-PL-EP-GEN SOul-3PL elle-li-mba-dit nat'e-yit. live-RES-PST.REP-3PL there-LOC.ADV

'Dies sind keine Menschen, dies sind die Seelen gestorbener Menschen, die dort leben.'

(ChDN_1983_TaleBrownies_flk_2, S: Vasjugan)

a. Qar'i-man wassa-l'ewl'e qwan-ni aw-i-r-kund'äl-o-k. morning-PROL get.up-CVB go.away-AOR.3SG eat-EP-FRQ-PTCP.CAR 'Am Morgen stand er auf und ging los, ohne zu essen.' (KMS_1966_TwoSisters_flk_24, S: Middle Ket')

b. Orap-so-j mergi tinwu-kund'älak force-INSTR-ADJZ wind know-PTCP.CAR-EP-ADV mita-lti- $\eta \quad$ onnän. reach-INCH-INT.PF-3SG.S up-LOC.ADV

'Ein starker Wind rauschte unbemerkt in der Höhe.' (MPF_1964_Birjuk_trans_23, S: Upper Ket')

a. Üd-e-p čače-sodi koček. water-EP-ACC go-PTCP.NEC much-ADV

'Zum Wasserholen gibt es viele.' (NN_1855_Hero1_song_83, Z/S: Tym/Middle Ob)

b. Soc'ka ap-sod'e au-r-na-q, par'in'te mi good eat-PTCP.NEC eat-FRQ-AOR-3DU.s bitter thing $\bar{e} r-a-k$. drink-AOR-3DU.S

'Sie essen gutes Essen, trinken Wodka (wrtl.: bitteres Ding).' (PMP_1961_ForestWoman_flk_378, S: Middle Ob)

c. Man kuatšo-d tïri narot-mə am-na-bba, kai-nē 1SG city-LOC.ADV full people-1SG eat-DRV-DUR.3SG what-DAT/ALL ap-sodi t'ängu-a-n. eat-PTCP.NEC NEG.EX-AOR-3SG.S

'Die Menschen in meiner Stadt wollen essen, etwas zu Essen fehlt.' (NN_1913_Itja_flk_15, S: Lower Chaja)

\subsubsection{Nominalphrase als Modifikator}

Neben einfachen Nomen können komplexe Nominalphrasen als Attribut innerhalb einer Nominalphrase auftreten. Die Funktion attributiver Nomen und/oder Nominalphrasen ist in der Regel die der Possession. Im Selkupischen treten typischerweise Genitiv- und Lokativattribute auf. 


\section{Phrasentypen}

\section{Nominativ-NP}

Unmarkierte Nomen erscheinen als Teil von Komposita, bei denen eine Spezifizierung des Kopfes stattfindet. Das Nomen wird hier adjektivisch gebraucht und als adjektivisches Attribut verwendet 13 . Im Korpus kommen sie nur selten vor, doch gibt es Beispiele, wie in (33a). Darüber hinaus können modifizierende NPs im Nominativ komitativische Funktion haben. Der nominale Modifikator ist hierfür mit dem Konnektiv-Reziprok-Suffix sowie entweder mit dem Plural oder, wie in (33b), mit dem Dual markiert (zu Komitativ-NPs siehe unten).

a. Taw [kibajče čel'egga] $]_{N P}$ mat pamošn'ijok.

this boy calf 1sG assistant

'Dieses kleine Kalb (wrtl.: Jung-Kalb) (ist) mein Assistent.'

(TTD_1964_CalfHare_flk_27, Z: Tym)

b. Eli-mpa-k warki-mba-k [era baja-sa-q] $]_{N P \text {. }}$

live-PST.REP-3DU.S live-PST.REP-3DU.s old.man old.woman-CRC-DU

'Es lebten und wohnten ein alter Mann mit seiner Frau.'

(PMP_1961_Fairytale_flk_1, S: Middle Ob)

\section{Genitiv-NP}

Eine Genitiv-NP erscheint immer vor dem Kopf. In beiden Dialektgruppen finden sich die beiden Genitivsuffixe $-t$ und $-n$. Die Beispiele (34a) und (34b) zeigen belebte Kopfnomen und belebte Genitiv-Modifikatoren. Mittels einer Genitiv-NP können auch räumliche Relationen wiedergegeben werden, wie in (34c). Der Kopf einer solchen NP signalisiert dann ,a spatial relation relative to the noun which encodes this relation." (Grenoble (2014: 124). Für die entsprechenden Beispiele wird der Begriff relationale Nomen verwendet, die Struktur als Adpositionalphrase analysiert, da diese Nomen in den meisten Fällen räumliche Relationen wiedergeben und nur noch vereinzelt mit der ursprünglichen lexikalischen Bedeutung verwendet werden. Eine detaillierte Betrachtung erfolgt in 2.7.2 auf Seite 68.

a. Azì-t $\bar{\varepsilon} \breve{z} a-l-g u-m b a: \quad$ [İde-n nenn'a-t $]_{N P}$ mat father-3SG say-RES-ITER-PST.REP.3SG Itja-GEN sister-3SG $1 \mathrm{SG}$ $n \overline{\mathcal{E}}-m$, Ide mat $\overline{\bar{l}}-m$. daughter-1SG, Itja 1SG son-1SG

'Der Vater sagte: Itjas Schwester (ist) meine Tochter, Itja (ist) mein Sohn.' (ILP_1981_ItjaOneEyedDevil_flk_13, S: Middle Ob)

b. Ulyo [ära-n n'e-p] $]_{N P}$ kwen-ni-mba-d, ice old.man-GEN daughter-ACC go.away-TR-PST.REP-3SG.O, kundak-ti kwen-ni-mba-d. far-ABL.ADV go.away-TR-PST.REP-3SG.O

'Das Eis trug die Tochter des Alten fort, trug sie weit fort.' (ChDN_1983_GirlAndIce_flk_10, Z: Vasjugan)

${ }^{13}$ Dies ist möglich, da in uralischen und auch altaischen Sprachen ursprünglich kein Unterschied zwischen den beiden Kategorien bestand (vgl. Fokos-Fuchs 1962: 66). 
c. Na [šōqor-t pār-o-nd] ${ }_{N P}$ siga-l-ba-dit.

this stove-GEN top-EP-ILL climb-INCH-PST.REP-3PL

'Sie kletterten in diesen Ofen14.'

(TMR_1981_Robbers_flk_35, S: Middle Ob)

\section{Adessiv-NP}

Auch Adessivattribute erscheinen vor ihrem nominalen Kopf. Hierbei kann das Attribut ein Nomen oder ein Pronomen sein. Insgesamt kommen Adessivattribute viel seltener als Genitivattribute vor. In (35a) ist sowohl der Kopf der Nominalphrase ein Nomen (HAJOUND - 'eye.PROL') als auch der Modifikator (ČUMBENĒNAN - 'wolf.ADE'). In den Beispielen (35b) und (35c) sind die Adessivattribute Personalpronomen der dritten Person Singular bzw. Plural, der nominale Kopf köda lilat - 'Rippenknochen' ein Kompositum.

a. Onక̌ kak kjek zašurši-t pomāga-he, što [čumbenē-nan himself how very start.rustling-3sG.o paper-INSTR, what wolf-ADE haj-o-und] $]_{N P}$ n'ary tü waš-q-wan-nā-i-t.

eye-EP-PROL red fire rise-ITER-DRV-AOR-EP-3SG.O

'Er machte so ein lautes Geräusch mit dem Papier, dass sich rotes Feuer in den Augen des Wolfes ausbreitete.'

(TTD_1964_CalfHare_flk_35, Z: Tym)

b. [Tab-d-i-nan tï̈ $]_{N P}$ öro-mba.

3SG-PL-EP-ADE fire disappear-PST.REP.3SG

'Ihr Feuer verschwand.'

(ChDN_1983_MistressOfFire_flk_37, Z: Vasjugan)

c. Na pit'ä ïjsel'c'e po-n ippa-n qa-ni t'eres', [tab-i-nan

this pike? year-ILL lie-3SG.S river-DAT/ALL across, 3SG-EP-ADE

köda li-la-t $]_{N P} \quad \overline{a d a}-t$.

rib bone-PL-3SG see-3SG.O

'Dieser Hecht liegt seit ewiger Zeit über dem Fluß, man sieht seine Rippenknochen.'

(PMP_1961_Fairytale_flk_119, S: Middle Ob)

\section{Komitativ-NP}

Vor allem in folkloristischen Texten kommen auch Komitativ-Attribute vor. Eine Modifikator-NP im Komitativ kann im Selkupischen eine Gruppe aus zwei Familienmitgliedern bezeichnen. Der Modifikator erscheint nach dem nominalen Kopf und ist mit dem Komitativsuffix, hier als Variante -si, 15 markiert, wie in (36).

(36) Mi kwas-sa-j [warga pekka-p elma-se (pekka-li-ka-se) $]_{N P}$. 1DU kill-PST-1DU big elch-ACC child-COM (elk-DIM-DIM-COM)

\footnotetext{
${ }^{14}$ Der traditionelle russische Holzofen ist so geräumig, dass man hineinkriechen kann.

${ }^{15}$ Das Suffix -sä/-se bezeichnet sowohl den Komitativ als auch den Instrumental, der erstere wird bei belebten, der letztere bei unbelebten Nomen verwendet.
} 
'Wir töteten einen großen Elch mit einem Kind (wrtl.: Elchlein).' (ChDN_1983_MotherInLaw_flk_14, Z: Vasjugan)

\subsubsection{Multiple Modifikatoren}

Im Korpus finden sich nur wenige Beispiele, in denen multiple Modifikatoren verwendet werden, wie in (37a). Das direkt Objekt kündä - 'Pferd' wird mit dem Demonstrativpronomen $n a$ - 'dieses', der Negationspartikel assa und dem Adjektiv sō - 'gut' modifiziert. Beispiel (37b) enthält ein Numeral (tä - 'vier'), ein Genitiv-Nomen (sain - 'Auge') sowie zwei Adjektive (pijaj - 'Eulen-' und ātaj - 'Rentier-') als Modifikatoren des nominalen Kopfes qorr - 'Hengst'. Aufgrund der geringen Anzahl von Belegbeispielen lässt sich keine konkrete Aussage über die Reihenfolge und eine Hierarchie der Modifikatoren machen. Das Demonstrativpronomen erscheint aber tendenziell vorn.

a. I ina assa sō kündä- $]_{N P}$. and take-PST-3SG.O this NEG good horse-ACC 'Und er nimmt dieses nicht gute Pferd.' (AGS_1968_FairytaleSnake_flk_79, S: Middle Ket')

b. [Tä sai-n pija-j āta-j qorra $]_{N P}$ ti-le qwässa-n-d four eye-GEN owl-ADJZ reindeer-ADJZ stallion fly-CVB iron-GEN-3SG ollo-nd swängi-r-i-ldä- $\eta$. head-ILL stick.through-FRQ-EP-INT.PF-3SG.S

'Ein vieräugiger Eulen-Rentier-Hengst kam geflogen, steckte sich in das Schaberköpchen hinein.'

(SVG_1964_IitekaPineweldju_flk_7, Upper Ket')

\subsubsection{Elliptische Phrasen}

Elliptische Phrasen bilden den dritten Phrasentyp, der nominale Strukturen aufweist. Dryer (2007: 37) definiert sie „as involving ellipsis of a head noun, that is, as involving a noun that is present at some level of structure but which is not expressed overtly“. Der (pro)nominale Kopf kann demnach auf der Satzoberfläche komplett fehlen (Das erste Brötchen aß ich, das zweite [...] fiel herunter.) oder der Modifikator einer $\mathrm{NP}$ wird zu ihrem Kopf. Im Belegmaterial finden sich Beispiele, in denen Demonstrativpronomina bzw. Numeralia als Modifikator einer NP zu deren Kopf werden. Dabei können die Modifikatoren, je nach Nominalphrase, unterschiedliche Numerus- und Kasusmarkierungen des ursprünglichen Kopfnomens tragen: In (38a) ist das fehlende Kopfnomen das Subjekt, der Modifikator $n \bar{a}-$ 'dieses' erscheint unmarkiert. In (38b) erscheint das Demonstrativpronomen $n a$ - 'dieses' mit Instrumentalmarkierung als indirektes Objekt, während es in (38c) als direktes Objekt, aber unmarkiert verwendet wird. Beispiel (38d) schließlich zeigt das Demonsrativpronomen taw - 'dieses' als direktes Objekt mit dem Akkusativsuffix $-m$. 
a. Nā-la kit'-wat-ple kur-a-nna-t.

this-PL get.frightened-DRV-CVB run-EP-AOR-3SG.O

'Diese erschraken und rannten weg.'

(SEV_1981_SisterBrother_flk_49, S: Middle Ob)

b. Na-ze šjōšqu-la ela-ku-mba-dit.

this-INSTR Selkup-PL live-ITER-PST.REP-3PL

'Mit Diesem lebten die Selkupen.'

(ILP_1981_HowSelkupLivedBefore_nar_8, S: Middle Ob)

c. Nänni kuld'i-na na qwäd'i-t.

then which-INDEF this leave-3SG.O

'Dann verließ er Diese irgendwie.'

(AGS_1968_FairytaleSnake_flk_41, s: Middle Ket')

d. Na qum t'ära-n: man taw-a-m i-tča-m.

this person say-3SG.S 1SG this-EP-ACC take-FUT-1SG.O

'Dieser Mensch sagt: Ich nehme Dieses.'

(AGS_1968_FairytaleSnake_flk_78, S: Middle Ket')

In den Beispielen (39a) und (39b) sind modifizierende Numeralia Kopf der NP, okkirim - 'einer.ACC' in (39a) und sadayun - 'zwei.LOc' in (39b). Auch sie tragen die Kasusund Numerusmarkierung des ursprünglichen Kopfnomens.

a. Lōz-la aj okkir-i-m üdi-mba-t devil-PL again one-EP-ACC send-PST.REP-3PL

'Die Teufel haben wieder Einen geschickt.'

(SEV_1981_SisterBrother_flk_60, S: Middle Ob)

b. Te taddi-n amda-lta, a me sədə-yun qwa-žaj

2PL here-ADV sit-IMP.2PL, but 1PL two-LOC go.away-1DU

'Ihr sitzt hier, aber wir Zwei gehen.'

(PVD_1961_FarmAssault_flk_33, S: Chaja)

\subsection{Negierte Nominalphrasen}

Bei der Betrachtung negierter Strukturen wird der Fokus auf die nordselkupischen Dialekte ausgedehnt. Dies erfolgt vor dem Hintergrund innerselkupischer Unterschiede, die nach Ansicht der Verfasserin eine erweiterte Betrachtung notwendig und sinnvoll macht. Die Beschreibung negierter Strukturen nimmt teilweise Bezug auf Wagner-Nagys ausführliche Beschreibungen (vorrangig) nordselkupischer Negationsstrategien (Wagner-Nagy 2011, Wagner-Nagy 2015), indem sie diese mit Korpusbeispielen belegt und ggf. auch Unterschiede feststellt 16 . Wagner-Nagys

\footnotetext{
${ }^{16}$ Wagner-Nagys Arbeit basiert auf dem typologischen Rahmen, den Miestamo (2005a), Miestamo (2005b) verwendet, indem er die Symmetrie bzw. Asymmetrie negierter Strukturen erfasst.
} 
Ausführungen werden so insbesondere mit Beispielen aus den zentralen und südlichen Dialekten ergänzt. Neben Wagner-Nagy beschäftigen sich auch einige Arbeiten Kovylins mit selkupischer Negation (S. V. Kovylin 2015, S. Kovylin 2015, S. Kovylin 2016).

In Übereinstimmung mit Dixon wird Negation in der vorliegenden Arbeit als „a term in a two-member polarity system \{positive, negative\}" (Dixon 2012: 90) betrachtet. Dabei ist die affirmative Variante unmarkiert. Affirmative und negative Sätze können sich lediglich hinsichtlich des Vorhandenseins eines Negationsmarkers im negativen Satz unterscheiden (symmetrischer Negationstyp bei Wagner-Nagy (2011: 64)). Oder die Unterscheidung kann zusätzlich auch im finiten Element bestehen (asymmetrischer Negationstyp bei Wagner-Nagy (2011: 54)). In den nordsamojedischen Sprachen wird ein Negationsverb zum Ausdruck der Standardnegation 17 verwendet (asymmetrischer Negationstyp). Das Selkupische verwendet hiervon abweichend eine Negationspartikel (ǎšs bzw. deren Varianten), die unmittelbar vor der finiten Verbform erscheint und gehört damit zum symmetrischen Negationstyp wie in Beispielen (40a) und (40b).

a. sugulže kuačo-nd üda-p.

home city-ILL let.go-1SG.O

'Ich lasse ihn zurück in die Stadt gehen.'

(NN_1855_Hero2_song_188, Z/S: Tym/Middle Ob)

b. Tab tan aya üde-nži-d.

3SG away NEG let.go-IPFV-3SG.O

'Er lässt ihn nicht gehen.'

(ChDN_1983_GirlAndIce_flk_7, Z: Narym)

Tabelle 8 zeigt die im Korpus belegten Negationswörter inkl. der Varianten und ihr Vorkommen in den einzelnen Dialektgruppen. Neben der Negationspartikel ašša kennt das Selkupische weitere Negationswörter zum Ausdruck der Negation. Die aus dem Russischen entlehnte Negationspartikel n'e bzw. $n$ 'i ist zwar in allen Dialektgruppen belegt, wird aber im Nordselkupischen ausschließlich zur Bildung von NPIs (negative polarity items) sowie zum Ausdruck der Doppelkonjunktion weder ... noch verwendet (siehe Abschnitt 2.2.1 unten). In den zentralen und südlichen Dialekten wird die Partikel auch zur Negation von Verbphrasen benutzt (siehe Kapitel 2.9). Die Partikel č '̌̈̈ $k \dot{i}$ ist aus der Form der 3. Person Singular des negativen Existenzialverbs čǟ $k \dot{z}$ - 'NEG.EX' entstanden. Ihre Verwendung ist nur in den nördlichen Dialekten belegt.

Die Negationspartikel $i k \dot{t}$ wird in allen Dialektgruppen ausschließlich in Imperativsätzen verwendet (Beispiele in Kapitel 2.9).

Alle Dialektgruppen verwenden das negative Existenzialverb čä̈jki bzw. čang(w)a, t'ang(w)a oder t'ägg(w)a. In den Norddialekten kann auch das negative Existenzial-

\footnotetext{
${ }^{17}$ Mit dem Terminus Standardnegation ist die Negation im Minimalsatz gemeint. Ein Minimalsatz besteht aus einem Prädikat und den notwendigen Argumenten, jedoch ohne Komplemente.
} 
verb in Kombination mit einer nominalisierten Verbform in einer präteritalen Umgebung zum Ausdruck der Standardnegation verwendet werden. Eine genauere Betrachtung erfolgt in Kapitel 4.4.2. Ebenfalls dort wird die von Bekker (1995b: 237ff.) für die Ob-Dialekte beschriebene Partikel n'etu betrachtet, die im Textkorpus nur in zentralen Dialekten verwendet wird und dort als negatives Existenzialverb interpretiert erscheint.

Tab. 8: Negationswörter im Selkupischen

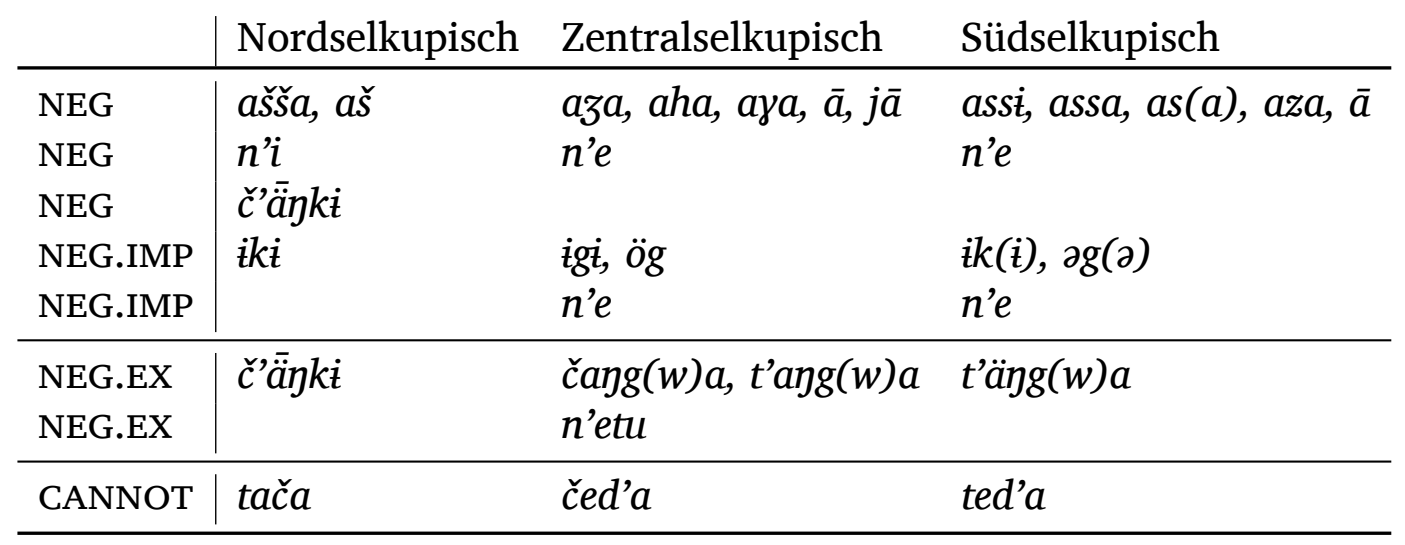

\subsubsection{Negierte nominale Köpfe}

Zu Beginn des Kapitel (2.1.1 auf Seite 19) wurde bereits beschrieben, dass unter dem Begriff nominale Köpfe sowohl nominale als auch pronominale Köpfe betrachtet werden. Beide können negiert werden, sind aber im Korpus lediglich vereinzelt belegt. Typischerweise wird die Verbalphrase statt der Nominalphrase negiert (vgl. Kapitel 2.9). Die Beispiele (41a) - (41c) zeigen negierte nominale Köpfe aus den zentralen und südlichen Dialekten. Zum Ausdruck der Standardnegation wird die Negationspartikel ašša bzw. eine ihrer Varianten dem nominalen Kopf unmittelbar vorangestellt. Nordselkupische Beispiele sind nicht belegt. Dort können nominale Köpfe mit der aus dem Russischen entlehnten Partikel $n ’ i$ negiert werden, wie in Beispiel 41d, allerdings nur im Sinne der Doppelkonjunktion weder ... noch. Beispiel $41 \mathrm{e}$ zeigt ein analoges Beispiel aus dem Zentralselkupischen.

a. Hir [aga hir] $]_{N P}$ ti elle pakti-ga. cow NEG cow here down jump-AOR.3SG

'Eine Kuh (oder) keine Kuh sprang hier runter.'

(ChDN_1983_BearCameIntoVillage_nar_6, Z: Vasjugan)

b. Kanž-la-n moqi-n pöngว-li-mba-n ol-laga, [as sledge-PL-GEN back-LOC.ADV roll-PLUR-PST.REP-3SG.S head-SING NEG qum] $]_{N P}$, a qu-n ol-laga-l.

person but GEN head-SING-ADJZ 
'Hinter dem Schlitten rollt ein Kopf, kein Mensch, sondern ein menschlicher Kopf.'

(PMP_1966_BodylessHead_flk_35, S: Middle Ob)

c. T'idam na qum qwän-nä n'ärne, tidam [assa toba-n] $]_{N P}$ now this person go.away-AOR.3sG forward now NEG leg-LOC.ADV a künda-n par-qin. but horse-GEN top-LOC

'Jetzt geht dieser Mensch weiter, nicht zu Fuß, sondern auf dem Pferd.' (AGS_1968_FairytaleSnake_flk_82, S: Middle Ket')

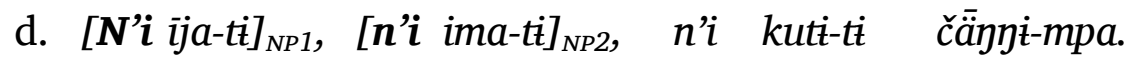
NEG child-3SG NEG woman-3SG NEG who-3SG NEG.EX-PST.REP.3SG 'Weder einen Sohn noch eine Frau, niemanden hatte er.' (NN_1977_OldMan_flk_2, N: Taz)

e. [n'ì čapte-t $]_{N P 1} \bar{a}$ kad-le, [n'ì haj-o-nd $]_{N P 2} \quad \bar{a}$ NEG tale-3SG NEG say-CVB NEG eye-EP-PL-3SG NEG qo-nža-r-d-le. sight-IPFV-FRQ-TR-CVB

'Weder [kann] eine Geschichte erzählt [werden], noch [können] die Augen [es] sehen.' (TTD_1964_Frog_flk_61, Z: Tym/Narym)

Häufiger als nominale Köpfe treten pronominale Köpfe negiert auf, hauptsächlich Interrogativpronomen. Dabei wird (vor allem im Nordselkupischen) die Negationspartikel $n$ 'i verwendet werden. In den Beispielen $42 \mathrm{a}-42 \mathrm{c}$ werden die Interrogatipronomen kuti und qaj negiert, indem ihnen die Negationspartikel $n$ ' $i$ vorangestellt wird. In Beispiel $42 \mathrm{~b}$ ist sie mit dem Pronomen verschmolzen.

a. Mat mōt-qük [n'i kuti $]_{P R O N P}$ aš ičča.

1SG house-ILL/LOC/ABL.1SG NEG who NEG go.3SG

'Ich bin in meinem Haus, niemand geht hinein.'

(NN_197X_YoungBoy_flk_29, N: Taz)

b. nā l'aga baba-ge qai-p qambigūnende-[n'eyaj $]_{\text {PRONP }} m \bar{e}$-špu-gu. this Baba Yaga-COM what-ACC? NEG.what do-FUT-INF

'Was werde ich mit dieser Baba-Jaga machen - nichts [werde ich] machen.'

(SDP_1964_FairytaleBlackZar_flk_902, Z: Narym)

c. Tō-n ippa, [ni qaj $]_{P R O N P}$ am-ne-nč̀-t.

there-LOC.ADV lie.3SG NEG what eat-DRV-FUT-3SG.O

'Dort liegt er, nichts wird er essen.'

(NN_1879_Itja_flk_62, S: Chaja)

In den zentralen und südlichen Dialekten sind Beispiele wie in (42b) und (42c) eher selten. Hier werden Interrogativpronomen typischerweise mit Hilfe des emphatischen 
Klitikons naj und einer nachgestellten Variante der Negationspartikel $a \check{s ̌ s} a$ negiert, wie in den Beispielen (43a) - (43c). Häufig ist das Klitikon mit dem Interrogativpronomen verschmolzen wie in (43a) und (43c), teilweise auch die Negationspartikel und das Klitikon wie in (43b). Beispiel (43d) zeigt eine nordselkupische Konstruktion mit der dem Interrogativpronomen vorangestellten Negationspartikel $n$ 'i.
a. Tab-i-n-nan [kai-m $=\mathbf{n a j}]_{P R O N P}$ aga nide-r-ba-d.
3SG-EP-GEN-ADE what-ACC = EMPH NEG pity-FRQ-PST.REP-3SG.O
'Er sparte für sie nichts an.'
(ChDN_1983_HerosDaughter_flk_10, Z: Vasjugan)
b. I-de $[\mathbf{q a i}-\boldsymbol{m}-\boldsymbol{n}-\boldsymbol{a s}]_{\text {PRONP }} \quad$ m'e-ku-t.
son-3SG what-ACC = EMPH-NEG do-HAB-3sG.O
'Ihr Sohn tut nichts.'
(KMS_1963_Sluggard_flk_4, S: Middle Ket')
c. tep-i-la-n $\quad n \bar{e}-d \quad[q a j-m=n a j a]_{P R O N P} a \quad k \dot{t} d \dot{t}-k u-d$.
3SG-EP-PL-GEN daughter-3SG what-ACC = EMPH NEG say-HAB-3SG.O
'Ihre Tochter sagt nichts.'
(TET_1979_SnowGirl_flk_15, s: Middle Ket')
d. L'ōs-ira lajki-n'-n'a, nop [n'i qaj-i-m] $]_{P R O N P}$ ašša kati-ti.
devil-old.man cry-VBLZ-AOR.3SG god NEG what-EP-ACC NEG say-3SG.o
'Der teuflische Alte sachreit - Gott sagte nichts.'
(BEP_1973_Itja2_flk_12, N: Taz)

In der Regel wird die Negationspartikel der zu negierenden Komponente unmittelbar vorangestellt. Zur Negation topikalisierter Pronomen kann in allen drei Dialektgruppen die Negationspartikel ašša verwendet werden, belegt sind Beispiele für das emphatische Pronomen (Beispiele (44a) und (44c)) und das Personalpronomen der 2. Person (Beispiele (44b) und (44d)). Dabei wird die negierte Konstituente hervorgehoben bzw. betont (nicht DICH lasse ich gehen. ICH werde gehen).

(44) a. Na [ašša ontì $]_{P R O N P}$ porqi-ti.

this NEG himself clothing-3SG

'Dies ist nicht SEINE eigene Kleidung.'

(NN_1973_Shaman_flk_5, N: Taz)

b. [A tat $]_{P R O N P 1} \check{s} \ddot{a} r-\check{c} a-l \check{z}$ i-mba-l i $\left[\begin{array}{ll}\bar{a} & t \bar{e} k a\end{array}\right]_{P R O N P 2}$

NEG 2SG dress-DRV-INT.PF-PST.REP-2SG.O and NEG 2SG.DAT

n'inge-gu tāb-e-t qōbi-p.

take.off-INF 3SG-EP-GEN skin-ACC

'Nicht DU hast sie angezogen und es ist nicht an DIR, ihre Kleidung auszuziehen.'

(TTD_1964_Frog_flk_88, Z: Tym)

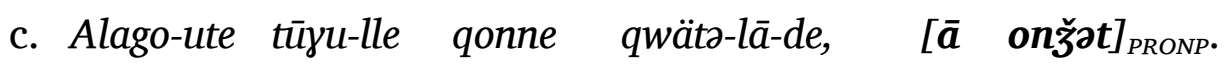
boat-PROL drag-CVB upwards begin-INCH-3PL NEG himself 
'Sie begannen, (ihre Sachen) vom Boot ans Ufer zu ziehen, nicht sie SELBST.'

(SDP_1964_FairytaleBlackZar_trans_1278, Z: Narym)

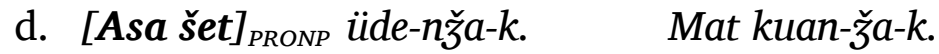

NEG 2SG.ACC let.go-FUT-1SG.o 1sG go.away-FUT-1sG.O

'Nicht DICH lasse ich gehen. Ich werde gehen.'

(NN_1855_Hero3_flk_29-30, Z/S: Tym/Middle Ob)

Wagner-Nagy argumentiert, dass es von der Form des Personalpronomens abhängt, ob es zwischen der Negationspartikel und dem finiten Verb stehen kann: die Kurzform der 1. und 2. Person des Personalpronomens als Objekt kann eingeschoben werden, die Langform hingegen nicht (Wagner-Nagy 2015: 51). Im Korpus kommen Kurzformen allerdings auch vor der Negationspartikel vor wie in den Beispielen (45a) und (45b). Eine andere mögliche Interpretation ist, dass die Negation in diesen Sätzen an den topikalisierten Personalpronomen angezeigt wird. Dazu passen die affirmativen Sätze in den Beispielen (45c) und (45d), in denen die Objekt-Personalpronomen tasta - '2SG.ACC' zur Topikalisierung vor dem Subjekt erscheinen.

a. It'e [s'im asse] sab-s-ku-s.

Itja 1sG.ACC NEG spring.out-DRV-HAB-PST.3SG

'Itja holt mich nicht heraus.'

(KKN_1971_Itja_flk_37, S: Upper Ket')

b. $\bar{I}-l \quad n a j$ [šinda jā] qos-ta-lā

son-2sG also 1SG.ACC NEG know-DRV-OPT.3SG

'Dein Sohn erkennt dich auch nicht ...'

(SDP_1964_FairytaleBlackZar_trans_1029, Z: Narym)

c. Kibba tibe-qum me-ndi-n $n$ t'äri- $\eta$ tasta nom small man-person do-INFER-3SG.S and say-3SG.S 2SG.ACC god

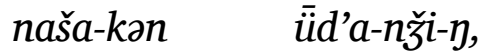
there-LOC.ADV let.go-FUT-3sG.S

'Der Jüngling tat es und sagt: DICH wird Gott dorthin gehen lassen, ...' (PMP_1961_Fairytale_flk_287, S: Middle Ob)

d. A tasta ese-l mega t'apti-za-n am-gu.

but 2sG.ACC father-2SG 1SG.DAT promise-PST-3SG.S eat-INF

'Aber DICH hat dein Vater mir zum Essen versprochen.'

(PMP_1966_BoyDevil_flk_43, S: Middle Ob)

\subsubsection{Negierte Modifikatoren}

Praktisch alle affirmativen Modifikatoren, die unter 2.1.2 beschrieben sind, können auch negiert auftreten. Für negierte Indefinitpronomen, Demonstrativpronomen und Partizipien als Modifikator einer Nominalphrase sind allerdings in keiner Dialektgrup- 
pe Beispiele belegt. Im Folgenden werden die negierten Modifikatoren mit Beispielen beschrieben.

\subsubsection{Negierte Possessivpronomen}

Enthält die NP ein Possessivpronomen als Modifikator wie in den Beispielen (46a) (46c), erscheint die Negationspartikel a $\breve{s ̌ s}$ bzw. eine ihrer Varianten vor dem zu negierenden Element, in diesem Fall vor dem Possessivpronomen als dem Modifikator der NP. In (46a) und (46b) ist der Modifikator das Possessivpronomen der 1. Person Singular, in (46c) das der 2. Person Singular. Nordselkupische Beispiele sind im Korpus nicht belegt.

a. Na [aha mat $\bar{i}-m]_{N P \text {. }}$

this NEG 1 SG son-1SG

'Dieser ist nicht mein Sohn.'

(KIA_2014_HeroKenga_trans_121, Z: Narym)

b. [Assi man tü-mbadi wattu-nga $]_{N P}$.

NEG 1SG come-PTCP.PST road-DIM

'Das ist nicht das von mir gegangene Weglein.'

(KMS_1966_TwoSisters_flk_13, S: Middle Ket')

c. Tāl [as tanani sü̈-di] $]_{N P}, \quad$ tau sü-di manani

that NEG 2SG.ADE sew-PTCP.PRS this 1SG.ADE sew-PTCP.PRS

nan'a-u- $n$.

sister-1SG-GEN

'Das ist nicht deine Näharbeit, diese Näharbeit ist die meiner Schwester.' (PMP_1961_ForestWoman_flk_224, S: Middle Ob)

\subsubsection{Negierte Interrogativpronomen}

Negierte Interrogativpronomen in der Funktion eines Modifikators einer NP sind im Korpus bis auf das nordselkupische Beispiel (47) nicht belegt. Wie für das Nordselkupische typisch, wird die aus dem Russischen entlehnte Negationspartikel $n$ 'i verwendet, um ein NPI (negative polarity item) zu formen. Der Kopf der Nominalphrase ist sümi - 'Geräusch'. Das negative Existenzialverb čäyka bildet einen negierten Existenzialsatz.

(47) Nīni [n’i qaj sümì] $]_{N P}$ čänka.

then NEG what noise NEG.EX.3SG

'Es gibt keine Geräusche.'

(AAI_1973_Okyle_flk_55, N: Taz) 


\subsubsection{Negierte Adjektive}

Adjektive als Modifikatoren einer NP werden negiert, um Antonyme auszudrücken. Beispiele sind in (48a) und (48b) für die zentralen und in (48c) und (48d) für die südlichen Dialekte belegt. Eine Ausnahme bildet Beispiel (48e) aus einer Übersetzung. Dort wird das vom Nomen mana - 'Dummkopf' abgeleitete Adjektiv manaj - 'dumm negiert'. Nordselkupische Beispiele sind nicht belegt.

a. Köy $[\overline{\boldsymbol{a}} \text { warqa } k \overline{\mathrm{t}}]_{N P}$.

Kenga NEG big river

'Die Kenga ist ein kleiner (wrtl.: nicht großer) Fluß.'

(KFN_1967_Mammoth_flk_6, Z: Tym)

b. $[\bar{A} \quad h w \bar{a} \quad \check{z} \bar{e} l]_{N P}$.

NEG good day

'[Es ist] ein schlechter (wrtl.: nicht guter) Tag.'

(SDP_1964_FairytaleBlackZar_trans_373, Z: Narym)

c. Nìn-a sō qorrä, a il'l'e-n ippa qaj-da

stand-AOR.3SG good stallion but bottom-LOC.ADV lie.3SG what-INDEF

[assa sō künda] ${ }_{N P}$.

NEG good horse

'Es steht ein schöner Hengst, aber auf dem Boden liegt irgendein nicht schönes Pferd.'

(AGS_1968_FairytaleSnake_flk_77, s: Middle Ket')

d. Üte-t nar štob [as qoj qu-la] $]_{N P}$ qüt'i-ja-dat, qoj qu-la let.go-3SG.O so that NEG rich person-PL be.ill-AOR-3PL rich person-PL qou-ne-dat.

rich-VBLZ-3PL

'Es geschah, dass sie armen (wrtl.: nicht reichen) Menschen krank wurden [und] die reichen Menschen reich(er).'

(KKN_1971_IvanAndGod_flk_34, S: Upper Ket')

e. Täp-jē-s-sa-n [assə mana-j tibə-qum] $]_{N P}$.

3SG-be-PST-3SG.S NEG fool-ADJZ man-person

'Er war kein dummer Mann.'

(ZIP_1964_Clock1_trans_25, S: Middle Ket')

\subsubsection{Negierte Numeralia}

Negierte Numeralia als Modifikator einer NP sind lediglich in übersetzten Texten belegt wie in den südselkupischen Beispielen (49a) und (49b). In beiden Fällen ist das Numeral okk - 'Eins' mit Hilfe der Negationspartikel assə negiert. 
a. [assa okka qamba-n] $]_{N P}$ pödar $\bar{i}-k u-z \dot{i}-t \quad$ mezowit NEG one spring-LOC.ADV Fedor take-HAB-PAST-3SG.O 1PL.PROL ondə-zimdizə sūru-j-gu piko-j pājgo-nda. himself-3PL.COM wild.animal-VBLZ-INF aspen-ADJZ forest-ILL

'Nicht in einem Frühling nahm uns Fjodor mit sich, um im Espenwald zu jagen.'

(KMS_1967_Hunt_trans_27, S: Middle Ket')

b. ..., nayo [assa okka časo-yinda] $]_{N P} \quad \overline{a m d a-d i ~ u ̈ t t-q a ̈ n . ~}$

then NEG one hour-ILL/LOC.3SG sit-3DU water-LOC

'weil sie nicht (erst) eine Stunde im Wasser sitzen.'

(KMS_1968_Burbot_trans_13, S: Middle Ket')

\subsubsection{Negierte Quantifizierer}

Das aus einem übersetzten Text stammende Beispiel (50) ist der einzige Korpusbeleg für einen negierten Quantifizierer als Modifikator einer NP. Zentral- oder nordselkupische Belege gibt es nicht.

(50) Tü-sa-y taw äto-nda müda-n manda-zi-l'ewl'e [assa qot't'i come-PST-3SG.S this village-ILL war-GEN pass.through-DRV-CVB NEG much po- $n]_{N P \text {. }}$ year-LOC.ADV

'Ich kam in dieses Dorf, nachdem der Krieg vorbei war, vor einigen (wrtl.: nicht vielen) Jahren.'

(KMS_1967_Hunt_trans_120, S: Middle Ket')

\subsubsection{Negiertes emphatische Pronomen}

Belege für negierte emphatische Pronomen als Modifikator einer NP finden sich lediglich im Nord- und Südselkupischen. Das nordselkupische Beispiel (51a) ist der einzige Beleg aus der Dialektgruppe, südselkupische Beispiele wie in (51b) stammen alle aus übersetzten Texten aus dem Ket'-Subdialekt.

a. Na [ašša onti porqi-tit] $]_{N P}, \quad \ldots$ this NEG himself clothing-3SG

'Diese ist nicht seine eigene Kleidung, ...'

(NN_1973_Shaman_flk_5, N: Taz)

b. tirqi-r-i-l'e $q \ddot{a} t-k u-z \dot{i}-t \quad$ [assa onda powi-n] $]_{N P}$. tremble-FRQ-EP-CVB say-HAB-PST-3SG.O NEG himself voice-INSTR2

'Er schüttelte sich und sagte nicht mit seiner eigenen Stimme.'

(KMS_1967_Antosja_trans_25, S: Middle Ket') 


\subsubsection{Negierte elliptische Phrasen}

Beispiel (52a) zeigt eine negierte elliptische Phrase. Das Kopfnomen qwäri - 'Krähe' fehlt hier und das Numeral oqqir - 'eins' übernimmt die Kopfposition inklusive der Kasusmarkierung des Nomens. Nord- und zentralselkupische Beispiele sind nicht belegt.

a. Na qum assi qwat-ni-t [oqqir-i-m n-asse]

this person NEG kill-AOR-3SG.O one-EP-ACC EMPH-NEG

'Dieser Mensch tötete nicht Eine.'

(AGS_1968_FairytaleSnake_flk_20, S: Middle Ket')

\section{Zusammenfassung}

Die selkupische Nominalphrase lässt sich wie folgt zusammenfassen: Der nominale (Beispiele (4) - (6)) oder pronominale (Beispiele (7a) - (7c)) Kopf der Phrase kann mit einfachen und komplexen Modifikatoren erweitert werden. Zu den Modifikatoren einfacher Nominalphrasen gehören neben Pronomen (Possessivpronomen in (10a) (16d), Indefinitpronomen in (19a) - (19a), Interrogativpronomen in (18a) - (18d), Demonstrativpronomen in (20) - (22b)) auch emphatische Pronomen wie in (24a) (24f) sowie in (25a) und (25b) auch Adjektive ((26a) - (26d)), Numeralia ((27a) (28c)), Quantifizierer ((29a) - (29f)) und Partizipien ((30a) - (30e)).

Mit verschiedenen Nominalphrasen, wie Nominativ-NPs ((33a) und (33b)), GenitivNPs ((34a) - (34c)), Adessiv-NPs ((35a) - (35c)) sowie Komitativ-NPs wie in (36) und multiplen Modifikatoren wie in (37a) und (37b) können komplexe Nominalphrasen erweitert werden. Attributive Relativsätze sind im Selkupischen durch russischen Einfluss entstanden und originär nicht vorhanden. In elliptischen Nominalphrasen ( $38 \mathrm{a})$ - (39b)) wird der nominale Kopf nicht overt ausgedrückt, stattdessen übernehmen unterschiedliche Modifikatoren Numerus- und Kasusmarkierungen des fehlenden Kopfnomens. Im Selkupischen erscheinen Demonstrativpronomen ((38a) - (38d)) und Numeralia ((39a) - (39b)) in elliptischen NPs.

Innerhalb einer Nominalphrase können sowohl nominale und pronominale Köpfe als auch Modifikatoren negiert werden. Von den in Tabelle 8 dargestellten Negationswörtern wird für nominale und einige pronominale Köpfe sowie für die meisten Modifikatoren die Negationspartikel ašša bzw. eine Variante verwendet. Eine Ausnahme bilden Interrogativpronomen, die in der Kopf- und der Modifikatorposition mit Hilfe der aus dem Russischen übernommenen Negationspartikel n'i negiert werden.

Die Beispiele (41a) - (41e) und (42a) - (45d) zeigen Korpusbelege für negierte (pro)nominale Köpfe aus den nördlichen, zentralen und südlichen Dialekten. Ebenso wie in der affirmativen NP können auch einzelne Modifikatoren negiert werden, wobei im Korpus nicht alle affirmativen Modifikatoren auch negiert belegt sind. Belege finden sich für Possessivpronomen (Beispiele (46a) - (46c)), Interrogativpronomen (Beispiel (47)), Adjektive (Beispiele (48a) - (48e)), Numeralia (Beispiele (49a) und 
(49b)), Quantifizierer (Beispiel (50)) sowie das emphatische Pronomen in den Beispielen (51a) und (51b). Beispiel (52a) schließlich zeigt eine negierte elliptische Phrase. Negierte Indefinitpronomen, Demonstrativpronomen und Partizipien als Modifikator einer Nominalphrase sind in keiner Dialektgruppe mit Beispielen belegt. 


\subsection{Adjektivphrase}

Adjektivphrasen enthalten ein attributiv, adverbial oder prädikativ verwendetes Adjektiv als Kopf. Eine Adjektivphrase besteht mindestens aus einem Adjektiv wie in den Beispielen (53a) und (53b). Die Adjektivphrase kann Derivationssuffixe enthalten wie der Adjektivmarker - $l$ und das Diminutivsuffix -čika in Beispiel (53c), letzteres in der Variante -žega, das zum Ausdruck von Komparation verwendet wird (zur ausführlichen Beschreibung der Komparation im Selkupischen siehe auch Bekker (1995b: 8ff.)).

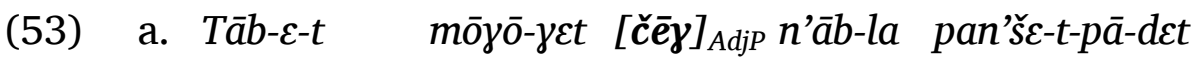
3SG-EP-GEN back-LOC white duck-PL swim-IPFV-PST.REP-3PL

$\bar{\varepsilon} t-k \varepsilon d$.

water-LOC

'Hinter ihr schwammen weiße Enten auf dem Wasser.'

(TTD_1964_Frog_flk_69, Z: Tym)

b. [Warga $]_{A d j P}$ n'an'a-det čanni-mba. big sister-3PL go.out-PST.REP.3SG

'Ihre große Schwester ging hinaus.'

(TMR_1967_Pönege_flk_20, S: Middle Ob)

c. $[\text { Kebe-l-žega }]_{A d j P} e$-ä-nd.

small-ADJZ-DIM be-AOR-2sG.S

'Du bist ziemlich klein.'

(NN_1855_Hero3_song_157, Z/S: Tym/Middle Ob)

\subsubsection{Modifikatoren}

Der adjektivische Kopf kann mit Hilfe von Modifikatoren erweitert werden. Hierzu zählen Adjektive, Adverbien, Partikeln und Adjunktorphrasen. Erweiterte Adjektivphrasen sind im Textkorpus nur selten belegt, am häufigsten werden modifizierende Adjektive verwendet. Für Adverbien und Partikeln gibt es oft nur einzelne Beispiele, die zudem häufig aus dem Russischen entlehnt sind.

\subsubsection{Adjektiv}

Ein adjektivischer Kopf kann mit einem Adjektiv erweitert werden. Da Modifikatoren dem Kopfnomen stets vorausgehen, kann angenommen werden, dass in (54a) pirgi 'steil' der adjektische Kopf ist, der vom vom Adjektiv waryi - 'groß' modifiziert wird. Im südselkupischen Beispiel (54b) ist der von einem Nomen abgeleitete adjektivische Kopf orsuj - 'stark' mit dem Adjektiv siwa - 'gut' modifiziert.
a. Čă̌aki-yit
[waryi pirgit $]_{\text {AdjP }}$ mad
par-git $e-j a$.

Chizhapka-LOC big high ravine river-GEN top-LOC be-AOR.3sG 
'Am Chizhapka gibt es einen großen steilen Abhang.'

(ChDN_1983_IdjasTown_flk_1, Z: Vasjugan)

b. Id'a orim-ba wargi- $\eta, \overline{\mathcal{E}}-z a \quad$ [siwa ors-u-j] $]_{\text {AdjP }}$

Itja grow.up-PST.REP.3SG big-ADV, be-PST.REP.3SG good force-EP-ADJZ

tibí-l-gum.

man-ADJZ.person

'Itja wurde erwachsen, er war ein guter starker Mann.'

(SMS_1980_ItjaForest_flk_24, S: Middle Ob)

\subsubsection{Adverb}

Adverbien als Modifikatoren kommen in zentral- und südselkupischen Texten vor. Die Beispiele (55a) und (55b) zeigen die Adverbien uruk - 'sehr' und nil'd'i - 'so' als Modifikatoren. Der Beispielsatz (55c) zeigt eine aus dem Russischen entlehnte Komparativ-Konstruktion, ešš krepč'e - 'noch stärker'. Die Komparativform des russischen Adjektivs krepkij - 'stark' ist mit dem ebenfalls aus dem Russischen stammenden Adverb eščo - 'noch' modifiziert.

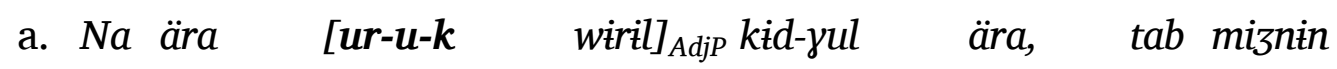
this old.man force-EP-ADV harmful magic-ADJZ old.man 3SG 1PL.ACC ab-la. eat-OPT.3SG

'Dieser alte Mann ist ein sehr böser Zauberer, er wird uns aufessen.' (ChDN_1983_Nikita_flk_22, Z: Vasjugan)

b. Okkir jedo-qin je-qa-n [nil'd'i kiba] $]_{\text {AdjP }}$ aur-ga. one village-LOC be-ITER-3SG.S so small boy-DIM

'In einem Dorf ist so ein kleiner Mann.' (PMP_1961_Fairytale_flk_16, S: Middle Ob)

c. Man tamd'el tēgga mē-ža-u qūduyo-m [eššo krepč'e] $]_{\text {Adjp }}$. 1SG today 2SG.DAT do-FUT-1SG.O pull.strap-ACC still strong.COMP 'Heute mache ich dir einen noch stärkeren Strick.' (PVD_1961_FarmAssault_flk_59, S: Chaja)

\subsubsection{Partikel}

In den Texten kommen Intensitätspartikeln wie in (56a) - (56c) und Negationspartikeln wie in (57a) als Modifikatoren von Adjektivphrasen vor. Die Partikel wet' 'noch' ist eine russische Entlehnung.
a. Mat-kinde [naj fa] $]_{\text {AdjP }} \varepsilon$-ppa.

house-LOC.3SG also good be-PAST.REP.3SG

'Im Haus war es auch gut.'

(ChDN_1983_HeroesDaughter_flk_17, Z: Vasjugan) 
b. N'äry tudo-n sei-n paqki-l-go-w, [näj tād-i-n] $]_{\text {AdjP }}$ red crucian-GEN eye-ACC dig-INCH-ITER-1SG.O also straight-EP-ADV je- $\eta$. be-3SG.S

'Ich grabe bei einer roten Karausche ein Auge aus, es ist auch gerade.' (PMP_1967_AboutItja_flk_72, S: Middle Ob)

c. Ma-nan [wet' kiba] $]_{\text {Adjp }} p a$ je-ta-n.

1SG-ADE still small knife be-IPFV-3SG.S

'Ich habe noch ein kleines Messer.' (PMP_1967_AboutItja_flk_65, S: Middle Ob)

d. Nì-ya sō qorrä, a il'l'e-n ippa qaj-da stand-AOR.3SG good stallion but down-LOC.ADV lie.3SG what-INDEF [assa sō] $]_{\text {AdjP }}$ künda. NEG good horse

'Es steht ein schöner Hengst, aber auf dem Boden liegt irgendein nicht schönes Pferd.'

(AGS_1968_FairytaleSnake_flk_76, S: Middle Ket')

\subsubsection{Multiple Modifikatoren}

Selten kommen auch komplexe Adjektivphrasen vor. Das bereits in Abschnitt (2.3.1.2) gezeigte Beispiel (57a) enthält das Adverb uruk - 'sehr' sowie die Adjektive wiril 'böse' und kidyul - 'zaubernd'. In (57b) kommen neben den Adjektiven fa - 'gut' und pirgi - 'hoch' auch die Partizipien kwedidel - 'fischend' und mencrtil - 'jagend' vor. Aufgrund der wenigen Beispiele für komplexe Adjektivphrasen lässt sich keine Aussage über eine Hierarchie treffen.

a. Na ära [uruk wiril kidyul $]_{A d j p}$ ära, tab miznin this old.man very harmful magical old.man 3SG 1DU.ACC ab-la. eat-OPT.3SG

'Dieser Mann ist ein sehr böser Zauberer (wrtl.: zaubernder alter Mann), er wird uns essen.'

(ChDN_1983_Nikita_flk_22, Z: Vasjugan)

b. mat bitta konze-r-na-l [fa kwedi-del pirgi 1SG as.if see-FRQ-AOR-2SG.O good be.beautiful-PTCP.PRS high menc-r-til $]_{\text {AdjP }} \quad k u-p$ hunt-FRQ-PTCP.PRS person-ACC 'Ich sehe einen guten, schönen großen Jäger (jagenden Menschen).' (ChDN_1983_HerosDaughter_flk_24, Z: Vasjugan) 


\subsection{Negierte Adjektivphrase}

Neben Nominal- und Verbalphrasen können auch Adjektivphrasen negiert werden. Im Textkorpus wird in den zentral- und südselkupischen Dialektgruppen die Negation von affirmativen Adjektiven verwendet, um ihr Antonym auszudrücken, wie in den Beispielen (62a) und (62b). Nordselkupische Beispiele sind nicht belegt.
a. $[\bar{A} \quad h w \bar{a}]_{A D J P} \breve{\breve{Z} e l . ~}$
'Ein schlechter (wrtl.: nicht guter) Tag.'
(SDP_1964_FairytaleBlackZar_trans_373, Z: Narym)
b. $I \quad \bar{l}-z \partial-t \quad$ na $[\text { assa } s \overline{0}]_{A D J P}$ kündä-m.
and take-PST-3SG.O this NEG good horse-ACC
'Und er nahm dieses schlechte (wrtl.: nicht gute) Pferd.'
(AGS_1968_FairytaleSnake_flk_78, S: Middle Ket')

\section{Zusammenfassung}

Insgesamt lässt sich festhalten, dass eine Adjektivphrase im Selkupischen mindestens ein Adjektiv enthält. Der adjektivische Kopf kann mit unterschiedlichen Modifikatoren erweitert werden. Am häufigsten werden Adjektive verwendet, seltener finden sich auch Adverbien oder Partikeln. Multiple Modifikatoren innerhalb einer Adjektivphrase kommen vor, jedoch lässt sich wegen der geringen Anzahl der Beispiele keine genauere Aussage über eine etwaige Hierarchie innerhalb derselben treffen.

Ein adjektivischer Kopf wird negiert, indem ihm die Negationspartikel ašša bzw. eine Variante vorangestellt wird. Belege sind vereinzelt in den zentralen und südlichen Dialekten zu finden, nordselkupische Beispiele finden sich im Korpus nicht. 


\subsection{Adverbphrase}

Der lexikalische Kopf einer Adverbphrase (AdvP) ist ein Adverb. Eine Adverbphrase enthält mindestens den Kopf. Adverbphrasen modifizieren Adjektivphrasen (siehe Kapitel 2.3), Verbphrasen oder den gesamten Satz. Die Beispiele (59a) und (59b) zeigen die kleinstmöglichen Adverbphrasen. Der Köpfe enne 'hinauf' und ponä 'hinaus' sind nicht modifiziert.

a. Amba-d [enne $]_{A d v P}$ waše-d'i-mba.

mother-3SG up rise-DRV-PST.REP.3SG

'Die Mutter stand auf.'

(ChDN_1983_MistressOfFire_flk_17, Z: Vasjugan)

b. Na qum čanžì $\eta$ [ponä] $]_{A d v P}$ māt-kanä, [...].

this person go.out-3sG.s outwards house-ABL

'Dieser Mensch geht aus dem Haus hinaus, [...].'

(AGS_1968_FairytaleSnake_flk_67, S: Middle Ket')

Typischerweise werden komparative Konstruktionen im Selkupischen (wie in vielen anderen uralischen Sprachen) innerhalb von Kopulasätzen mit einem adjektivischen Prädikat ausgedrückt (vgl. Abschnitt 4.1.3). Beispiel (60) zeigt eine Komparativkonstruktion, in der die Adverbphrase $s \bar{\eta} \eta$ - 'gut' komparatiert wird. Anstelle des typischerweise verwendeten adjektivischen Prädikats wird hier ein verbales Prädikat verwendet. Die Standard-NP wird mit dem Ablativ des Personalpronomens meynanni ausgedrückt.

(60) Tan ko-nžu-r-na-l mejnanni sō- $\boldsymbol{\eta}_{\text {AdvP. }}$.

2SG see-IPFV-FRQ-AOR-2SG.O 1SG.ADE good-ADV

'Du siehst besser als ich.'

(BNN_1971_EyesEars_flk_18, S: Middle Ket')

\subsubsection{Modifikatoren}

Der adverbielle Kopf kann nur eingeschränkt mit Hilfe von Negations-, bzw. Intensitätspartikeln modifiziert werden.

\subsubsection{Partikel}

Partikeln können verwendet werden, um Adverbphrasen zu modifizieren. Die Beispiele (61a) und (61b) zeigen die Negationspartikeln aya bzw. ass, Beispiel (61c) die Intensitätspartikel k'ek - 'sehr'. Die Personalendung am Verb in (61a) sollte eigentlich in der 1. Person Singular stehen.

(61) a. Mat [aya kundak-kit] $]_{A d v P}$ ta-nan šeki-ya-nd.

1SG NEG far-LOC.ADV 2SG-ADE spend.the.night-AOR-2sG.S

'Ich übernachtete nicht weit von dir.'

(ChDN_1983_ItjaStayedAlone_flk_32, Z: Vasjugan) 
b. Sābol', [ass sōwe- $\eta]_{\text {AdvP, }}$ ass mitte-ņ̌i- $\eta$.

truth NEG good-ADV NEG achieve-FUT-3SG.S

'Die Wahrheit, (es ist) nicht gut, er wird (es) nicht erreichen.'

(MMP_1964_Lgov_nar_104, S: Upper Ket')

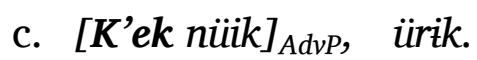

very sweet-ADV fat-ADV

'Es ist sehr süß, fett.'

(SAG_1984_StoryAboutLife_nar_27, Z: Narym)

\subsection{Negierte Adverbphrase}

Adverbphrasen werden auf die gleiche Art wie Adjektivphrasen negiert, indem aus affirmativen Adverben mit Hilfe der vorangestellten Negationspartikel ašša bzw. eine ihrer Varianten das negative Pendant gebildet wird. Im Korpus sind nur vereinzelt Beispiele belegt, wie in (62a) und (62b). Die von Bekker (1995b: 238) beschriebene Verschmelzung der Negationspartikel mit einem Adjektiv bzw. Adverb ist in Beispiel (62b) ebenfalls belegt.

(62) a. L'ōs-ira [ašša kuntì-k] $]_{A D V P} \bar{\varepsilon}-\eta j a$.

devil-old.man NEG long-ADV be-AOR.3SG

'Der teuflische Alte ist nicht weit entfernt.'

(AVA_1973_Ichakicha2_flk_97, N: Taz)

b. ... üdu-l-g-un [a-koče-ka] $]_{A D V P}$

drink-INCH-ITER-1PL neg-much-ADV

'... wir tranken ein bisschen.'

(PAV_NN_HowICatchBear_nar_97, Z: Tym)

c. [As kuntə- $\eta]_{A D V P} \bar{a} m t a-n, \ldots$

NEG long-ADV sit-3SG.S

'Er sitzt nicht lange, ...'

(NN_1879_Itja_flk_42, S: Chaja)

\section{Zusammenfassung}

Über die Adverbphrase im Selkupischen lässt sich festhalten, dass sie mindestens ein Adverb als Kopf enthält. Darüber hinaus kann sie mittels Partikeln modifiziert werden (Textbelege finden sich für Negations- und Intensitätspartikeln in den Beispielen (61a) - (61c)). Das negative Pendant einer Adverbphrase wird gebildet, indem ihr die Negationspartikel ašš bzw. eine Variante vorangestellt werden. Beispiele sind für alle drei Dialektgruppen belegt (Beispiele (62a) - (62c)) 


\subsection{Adpositionalphrase}

Der Kopf einer Adpositionalphrase ist eine Adposition. Bekker (1995b: 180ff.) und Helimski (1998: 571) unterscheiden zwischen nominalen Stämmen, die mittels eines Lokalkasus als Postposition fungieren sowie indeklinablen Postpositionen. Erstere werden in der Arbeit als „komplexe“, letztere als „einfache“ Postpositionen bezeichnet. Das pränominale Element erscheint in der Regel im Genitiv. Nicht alle von Bekker beschriebenen neun Postpositionen finden sich im Textkorpus. Mit Beispielen belegen lassen sich čombi - 'entlang', čanž - 'bis', migin - 'bei', čadi - 'anstatt' und tare - 'wie'. Für die Postpositionen kundì - 'während', kožatko - 'hinter, zusammen', čit - 'über, auf' und čare - 'über, nach' lassen sich keine Beispiele im Korpus finden. Dahingegen finden sich im Textkorpus die Postpositionen ugōn - 'vor, bis' (nur südselkupische Dialekte) und opti - 'mit' (zentral- und südselkupische Dialekte), die weder bei Helimski noch bei Bekker beschrieben sind.

Nachfolgend werden sämtliche Postpositionen und ihre Funktionen dargestellt, die sich mit Textbeispielen belegen lassen. Die Beschreibung beginnt mit den einfachen Postpositionen in Abschnitt 2.7.1, an die sich die komplexen Postpositionen in Abschnitt 2.7.2 anschließen. Den Schluss bildet eine Übersicht über Präpositionen in Abschnitt 2.7.3, die sich unter russischem Einfluss entwickelt haben.

\subsubsection{Einfache Postpositionen}

Im Textkorpus können für das Zentral- und Südselkupische sieben Postpositionen anhand von Beispielen belegt werden. Tabelle 9 gibt einen Überblick über die Postpositionen in den Dialektgruppen und die Art der Relation, die sie zu ihrem Bezugsnomen herstellen. Im Folgenden werden die einzelnen Postpositionen detailiert beschrieben und mit Beispielen illustriert.

Tab. 9: Postpositionen im Selkupischen

\begin{tabular}{llll}
\hline \multicolumn{2}{l}{ Dialektgruppe } & & Relation \\
Zentral & Süd & & \\
\hline čan & & 'bis' & temporal \\
& mijin & 'bei' & lokal, temporal \\
opti & opti & 'mit' & modal \\
tare & tare & 'wie' & komparativ \\
taet & t'at & 'anstatt, über' & modal, kausal, lokal \\
క̌omb & t'ombi & 'während, entlang' & lokal, temporal \\
& ugōn & '(da)vor' & temporal \\
\hline
\end{tabular}

čan

Die Postposition čan - 'bis' ist bei Bekker (1995b) als čanž beschrieben. Im Textkorpus kommt sie nur im zentralselkupischen Beispiel (63) vor. Die Postposition drückt eine 
temporale Relation aus, das unmittelbare Bezugsnomen pō - 'Jahr' erscheint unmarkiert.

(63) [Okkarkwilget po-yəndo helžaro pō čan $]_{A D P}$ mačča-ča-k. eleven year-ILL.3SG seventy year until hunt-TR-1SG.S

'Von elf bis siebzig habe ich gejagt.'

(MNN_1977_Hunt_nar_3, Z: Narym)

\section{mixin}

Die Postposition mizyin - 'bei'18 ist zwar auch bei Bekker (1995b: 180ff.) für die zentralund südselkupischen Dialekte beschrieben, kommt jedoch im Textkorpus in nur wenigen südselkupischen Belegen vor 19 . Diese lassen sich grammatisch nicht mehr eindeutig als Postpositionalphrase analysieren, da sie lediglich in Formen wie in (65a) - (65c) vorkommen. Dabei werden sowohl lokale wie in (65a), als auch temporale Relationen wie in (65b) und (65c) ausgedrückt.

a. I omda-di oqqa-miqin, äda-ľ̌i-l'e na sü-m.
and sit-3DU one-near, wait-INT.PF-CVB this dragon-ACC

'Und sie sitzen nebeneinander und warten auf diesen Drachen.'

(AGS_1968_FairytaleSnake_flk_92, S: Middle Ket')

b. Tapti qari-mizin poqi-n nado manni-mbi-gu. next morning-near net-ACC necessary look-HAB-INF

'Am nächsten Morgen muss man nach dem Netz schauen.' (TFF_1967_IdjaAldigaNet_flk_9, S: Middle Ob)

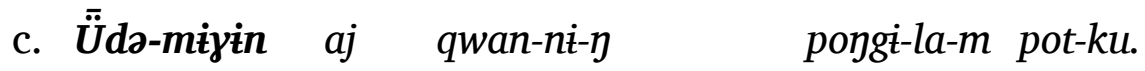
evening-near again go.away-AOR-3SG.S net-PL-ACC settle.net-INF

'Am Abend fuhr er wieder los, um die Netze zu stellen.' (PMP_1967_AboutItja_flk_14, S: Middle Ob)

\section{opti}

Die Postposition opti - 'mit' drückt modale Relationen aus. Beispiele gibt es in beiden Dialektgruppen, (66a) und (66b) stammen aus dem Zentralselkupischen, (66c) aus dem südselkupischen Ket'-Dialekt. Der Modifikator ist in allen Fällen ein belebtes Nomen, in (66c) ist er zusätzlich mit dem Possessivsuffix markiert.

$$
\text { a. } \mathrm{Mi} \quad \text { paja-n opti }{ }_{A D P} \text { mad'o-ndi kwes-sa-j. }
$$

1PL.DU old.woman-GEN with taiga-ILL go.away-PST-1DU

\footnotetext{
${ }^{18}$ Die Postposition ist historisch aus dem relationalen Nomen $m i$ - 'Ding, Sache' + LOC entstanden.

${ }^{19} \mathrm{Im}$ Nordselkupischen drückt die Postposition eindeutige lokale Relationen aus:
}

(64) $[T \partial p-\dot{i}-n \quad m \dot{t} q \dot{t} n]_{A D P} \varepsilon-p p a-n \dot{t} \quad i j a-t \dot{t}$.

3SG-EP-GEN near be-HAB-AOR.3sG son-3sG

'Sie hatte einen Sohn (wrtl.: Bei ihr war ein Sohn).'

(MIV_1977_Icha_flk_2, N: Upper Taz) 
'Ich ging mit der Alten in die Taiga.'

(ChDN_1983_MotherInLaw_nar_1, Z: Vasjugan)20

b. Okur kudä-t qwäl-la-ye šeda qu-t

one when-LOC.ADV go.away-OPT-3DU.s two person-3SG

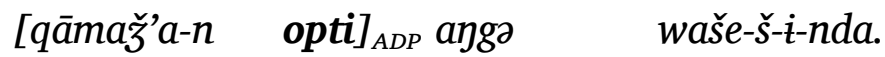

Kamacha-GEN with capercaillie fly-US-EP-INFER.3SG

'Manchmal, wenn zwei Menschen mit Kamacha gehen, fliegt ein Auerhahn.'

(JIF_1968_Kamacha_nar_81, Z: Tym)21

c. Kun $=n \dot{t} \quad$ ewe-sä-qqi $\quad$ pald'ü-ku-s-ti $\quad[\bar{i}-n-d$

where $=$ EMPH mother-COM-DU go-ITER-PST-3SG.O son-GEN-3SG

opti $]_{A D P}$.

with

'Irgendwohin ging die Mutter mit ihrem Sohn.'

(SVG_1967_Spring_nar_10, S: Upper Ket')22

tare

Die Postposition tare - 'wie' wird verwendet, um einen Vergleich auszudrücken23. Sie erscheint vor allem in südlichen Texten. In den Beispielen (67a) und (67b) erscheint die Postposition in den (Sprecher-) Varianten tarēdal bzw. tarēdik. Der Modifikator kann nominal wie in (67b) oder pronominal wie in (67c) sein. Regulär erscheint der Modifikator im Genitiv, wie in (67b) und (67c), in (67a) und (67d) tritt er unmarkiert auf.

a. Qudə=naj nil'd'ì $\bar{a} \quad$ čèla-mbā $[\text { mat tarēdal }]_{A D P}$ who $=$ EMPH so $\quad$ NEG give.birth-PST.REP.3SG 1SG like

or-hul.

force-ADJZ

'Niemand wurde so stark geboren wie ich.'

(SDP_1964_FairytaleBlackZar_flk_892, Z: Narym)

b. Na pēge [qu-t tarēdik $]_{A D P} \bar{\partial} \check{a} a-l-g w a: \quad$ mašep

this hazel.grouse persong-GEN like say-INCH-ITER.3SG 1SG.ACC ik t'āč-k-l'e-l.

NEG.IMP shoot-OPT-2SG.O

'Das Haselhuhn sagte wie ein Mensch: Schieß nicht auf mich!' (SEV_1980_HazelGrouse_flk_7, s: Middle Ob)

\footnotetext{
${ }^{20} \operatorname{Im}$ Original erscheint die PP zusammengeschrieben, ich analysiere das Beispiel als PP mit Modifikator-NP: [ADPP [NP paja-n] opti].

${ }^{21} \mathrm{Im}$ Original erscheinen okurkudät, šedəqut sowie die PP zusammengeschrieben, ich analysiere die PP wie folgt: [ADPp [NP qāmaž’a-n] opti].

${ }^{22}$ Im Original erscheint die PP zusammengeschrieben, ich analysiere sie hingegen wie folgt: [ADPP [NP i-n-d] opti].

${ }^{23}$ Mit Indefinitpronomen werden ebenfalls Vergleiche ausgedrückt, sie sind in Kapitel 4.1.1 näher beschrieben.
} 
c. A Pōnegessa träzni-mba-t, qai-m qade-špa-t İde, $i$ and Pönegessa sing-PST.REP-3SG.O what-ACC tell-IPFV1-3SG.O Itja and Pönegessa [tab-i-t tare $_{A D P}$ naj lä-ra.

Pönegessa 3SG-EP-GEN like also sing-FRQ-AOR.3sG

'Und Pönegessa singt, was Itja erzählt, und Pönegessa singt auch auf seine eigene Weise.'

(ITFF_1967_ItjaAldigaScale_flk_15, S: Middle Ob)

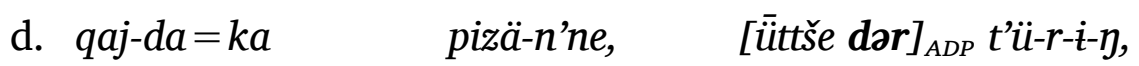

what-INDEF $=$ EMPH laugh-AOR.3SG child like cry-FRQ-AOR.3SG

[kana dor $]_{A D P} m \bar{u} d \dot{i}-\eta, \quad$ [qwärya dor $]_{A D P} q \bar{a} r \dot{r}-n$ 'e,

dog like bark-3sG.s bear like shout-AOR.3sG

süm-di-mba

noise-DRV-PST.REP.3SG

'Jemand lachte, weinte wie ein Kind, bellte wie ein Hund, brüllte wie ein Bär, blies.'

(KMS_1967_EagleOwl_nar_8, S: Middle Ket')24

t'āt

Die Postposition t'at - 'anstatt, über' drückt modale, kausale und lokale Relationen aus. Die wenigen Belege stammen hauptsächlich aus den südlichen Dialekten, Beispiel (68a) mit lokaler Funktion ist der einzige Beleg aus dem Zentralselkupischen. Die modale Relation findet sich vor allem in Ob-Dialekten, wie in Beispiel (68b), die kausale Relation wird in Ket'-Dialekten ausgedrückt, wie in (68c).

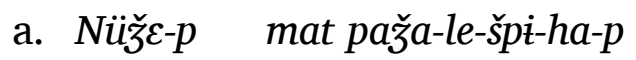
$[\text { Koldi-t tact }]_{A D P}$
grass-ACC 1SG chop-INCH-IPFV-AOR-1SG.O Ob-EP-GEN over
kiba-n-jur-get ([na malenakom $]_{\text {Codeswitching }}$
small-GEN-?-LOC (on small.PRÄPOSITIV
sor-u).

low.area.covered.by.water-?

'Ich mähe das Heu über dem Ob, auf einem kleinen niedrigen, mit Wasser bedeckten Gelände.'

(TMN_1994_Cow_nar_9, Z: Narym)

b. Mat [ī-t t'āt $]_{A D P}$ tēka $\bar{\varepsilon}-n \check{z} a-k$.

1SG son-GEN instead 2SG.DAT be-FUT-1SG.S

'Ich werde dir ein Sohn sein (an Sohnes statt).'

(SEV_1980_HazelGrouse_flk_8, S: Middle Ob)
c. N'ärnä-j t'ela-ndi-ze wes qrā-yən tšēntšu-za-tto
forward-ADJZ day-OBL.3SG-CRC all border-LOC say-PST-3PL
P'ödir-i-n d'ät.
Fedor-EP-GEN instead

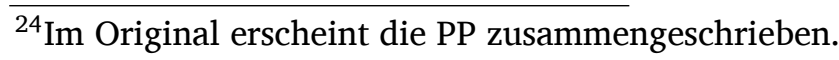


'Am nächsten Tag sprachen alle über Fedor,'

(KMS_1967_Hunt_nar_111, S: Middle Ket')25

\section{3̌omb / t'ombi}

Die Postposition žomb (zentralselkupisch) bzw. t'ombí (südselkupisch) - 'während, entlang, innerhalb' drückt lokale und temporale Relationen aus. Beispiel (69b) mit lokaler Funktion zeigt das einzige südselkupische Beispiel26. Der Modifikator ist dort regulär mit dem Genitiv markiert. Im zentralselkupischen Beispiel (69a) hingegen erscheint der Modifikator unmarkiert, die Postposition drückt eine temporale Relation aus.

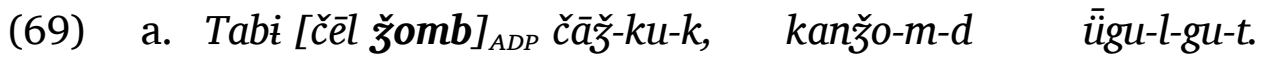
3SG day during go-ITER.3SG sledge-ACC-3SG pull-INCH-ITER-3SG.O 'Sie läuft den ganzen Tag, zieht den Schlitten.' (SAA_1984_MyGrandmother_nar_4, Z: Narym)

b. It'e üt'i-mb'i-ku- $\eta \quad$ [mada-m t'ombi $]_{A D P}$, asse Itja let.go-HAB-ITER-3sG.s house-GEN during NEG

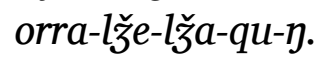
hold-TR-INT.PF-ITER-3SG.S

'Itja läuft im Haus herum, es gelingt [Loz] nicht, ihn zu fassen.' (KKN_1971_Itja_flk_15, S: Upper Ket')

\section{ugōn}

Die Postposition ugōn - '(da)vor' ist nur in südselkupischen Dialekten mit temporaler Funktion belegt. In zentralselkupischen Textep erscheint sie als Adjektiv mit der Bedeutung 'früher'. In den Beispielen (70a) und (70b) werden mit Hilfe der Postposition Zeitangaben ausgedrückt.

a. [tau-n-ugon] $]_{A D P}$ qaj tan-nani nan'a-l süd-ə-r-ə-qo-s this-GEN-before what 2SG-ADE sister-2SG sew-EP-FRQ-EP-ITER-PST.3SG mega.

\section{SG.DAT}

'Bis dahin, was (sollte) deine Schwester für mich genäht haben?' (PMP_1961_ForestWoman_flk_270, S: Middle Ob)

b. atšatšipsa pā-qa-m [taw-a-n ukon $]_{A D P}$ sesolukonney drill knife-DIM-ACC this-EP-GEN before in.case.of.danger pän-a-lde-ku-sa-m. put-EP-INT.PF-ITER-PST-1SG.O

'Ich habe davor mein Bohrmesser für den Fall der Gefahr (bereit) gelegt.' (SVG_1964_IitekaPineweldju_flk_45, S: Upper Ket')

Zusammenfassend lassen sich für die zentralen und südlichen Dialekte sieben ein-

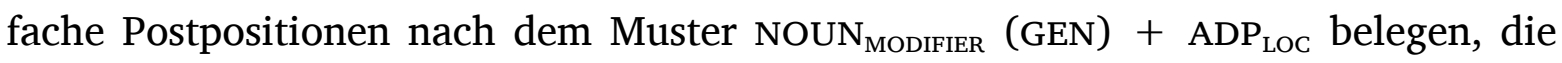

\footnotetext{
${ }^{25}$ Im Original erscheint die PP zusammengeschrieben.

${ }^{26}$ Die Postposition wird dort vor allem als Adjektiv mit der Bedeutung 'lang' verwendet.
} 
unterschiedliche, teilweise auch mehrere Relationen ausdrücken können. Temporale Relationen drücken čan - 'bis', mizin - 'bei', క̌omb bzw. t'ombi் - 'während, entlang, innerhalb' und ugōn - '(da)vor' aus. Lokale Relationen können mit Hilfe von mizin 'bei', t'at - 'über' und క̌omb bzw. t'ombi - 'während, entlang, innerhalb' ausgedrückt werden. tare - 'wie' wird verwendet, um komparative Relationen zu beschreiben. Die Postpositionen opti - 'mit' und t'at - 'anstatt, über' haben modale Funktion, letztere auch kausale. Einige Postpositionen erscheinen nur in zentralen Dialekten, wie čan, oder nur in südlichen, wie mizin und ugōn, andere Postpositionen sind in beiden Dialektgruppen belegt, wie opti, tare, t'at und ร̌omb im Zentralselkupischen bzw. t'ombi im Südselkupischen.

Neben einfachen Postpositionen sind im Selkupischen eine Reihe von komplexen Postpositionen belegt, die sich aus relationalen Nomen entwickelt haben (vgl. auch Kahr (1975: 26), Harder (2017: 165ff.)) und im Folgenden beschrieben werden. 


\subsubsection{Komplexe Postpositionen}

Komplexe Postpositionen bilden die zweite Gruppe. Sie bestehen aus Konstruktionen, die ein relationales Nomen enthalten. Der (pro)nominale Modifikator ist, wie bei den einfachen Postpositionen, in der Regel mit dem Genitiv markiert (NOUN MODIFIER $_{\text {(GEN) }}$ $+\mathrm{ADP}_{\mathrm{LOC}}+\mathrm{Affi}_{\mathrm{LOC}}$ ). Das (relationale) Kopfnomen hingegen ist mit einem Lokalkasus (Dativ/Allativ, Lokativ, Illativ, Ablativ, Prolativ) markiert. Daneben kennt das Selkupische eine Reihe lokaler Adverbialkasus, die sowohl an Adverbien als auch an Nomen auftreten können. Tabelle 10 zeigt die unterschiedlichen Adverbialkasus, $P R O L . A D V$ ist nur in zentralen Texten belegt.

Tab. 10: Adverbialkasus im Zentral- und Südselkupischen

\begin{tabular}{lll}
\hline & Zentral & Süd \\
\hline LOC.ADV & $-n,-q \dot{i} n$ & $-n,-q \dot{i} n$ \\
ABL.ADV & $-n \dot{t},-q \dot{i n} \dot{t}$ & $-n \dot{t},-q \dot{i} \dot{i}$ \\
PROL.ADV & $-u t \varepsilon$ & \\
\hline
\end{tabular}

Helimski (1998) nennt zwanzig Nominalstämme, die als Adposition im Selkupischen verwendet werden können, ohne sie jedoch genauer zu beschreiben, im Korpus gibt es Belege für zwölf relationale Nomen. Tabelle 11 aus Harder (2017) gibt einen Überblick über die relationalen Nomen im Selkupischen und die semantischen Felder, aus denen sie stammen 27 .

Tab. 11: Relationale Nomen und ihre semantischen Felder

\begin{tabular}{|c|c|c|c|c|c|}
\hline \multicolumn{3}{|c|}{ Körperteile28 } & \multicolumn{3}{|c|}{ Räumliche Umgebungen } \\
\hline oli & (*olə) & 'Kopf' & tōp & (*tōp) & 'Ecke' \\
\hline$q \bar{a} t$ & (*qāt) & 'Stirn' & par & ("pīrə) & 'Oberseite' \\
\hline saj & (*səjmä) & 'Auge' & tibo & (*topi / *täpc) & 'Ende' \\
\hline$a q$ & (*än) & 'Mund' & ìla & (*ilə) & 'Boden' \\
\hline s'ünc'ə & (*künje) & 'Magen, Inneres' & puక̌o & ("pūčə) & 'Rohr, Inneres' \\
\hline & (*kåj) & 'Seite, Rippe' & & & \\
\hline moqa & (*məkå) & 'Rücken' & & & \\
\hline
\end{tabular}

\section{oli}

oli - 'Kopf' wird verwendet, um die lokalen Relationen 'oberhalb' und 'über' auszudrücken. Beispiele finden sich ausschließlich in südlichen Dialekten, wie in (71),

\footnotetext{
${ }^{27}$ Das in der Tabelle von Harder (2017: 157) vorkommende relationale Nomen po - 'Außenseite' wurde weggelassen, da es als einziges nicht als Adposition vorkommt, sondern hauptsächlich als lokales Adverb.

${ }^{28}$ Die meisten protosamojedischen Formen sind Janhunen (1977) entnommen, die Formen *qāt, *tōp, "pūča und "pira stammen aus Alatalo/Donner/Sirelius (2004).
} 
in zentralen Dialekten kommt das relationale Nomen in Genitivkonstruktionen vor, nicht aber als Adposition.
(71) Sūru-l
tāraē n'ün'ü-ka-y [qi-n
ollo-nda $]_{P P}$
wild.animal-ADJZ hair small-DIM-ADV river-GEN head-ILL
t'äz̆a-ku-t
throw-ITER-IMP.2SG.O
'Wirf das Fell nach und nach in den Oberlauf des Flusses hinein.'
(KNI_1964_ItjaCapeOfWorms_flk_41, S: Upper Ket')

\begin{abstract}
qāt
$q \bar{a} t$ - 'Stirn' kann als Adposition 'vor' verwendet werden wie in (72a) und (72b). Im zentralselkupischen Beispiel (72a) ist der Modifikator das Personalpronomen der 1. Person, es erscheint unmarkiert. Das südselkupische Beispiel (72b) zeigt die Postposition in der Variante kuto mit nominalem Modifikator im Genitiv.
\end{abstract}
parza-lā:
Sifka Purka, tż

a. Čistoj pōle-nd čanžว-lā, clean field-ILL go.out-OPT.3SG shout-OPT.3SG Sifka-Burka here
tö-špa- $q$,
[mat qat-qöq $]_{P P}$ no-le-ě̌ik!
come-IPFV-IMP.2SG.S 1SG forhead-LOC stand-INCH-IMP.2SG.S

'Er ging auf das leere Feld hinaus (und) schrie: Sifka-Burka, komm hierher (und) stell dich vor mich!'

(SDP_1971_FairytaleBlackZar_trans_953, Z: Narym)

b. It'e aqqol [peči $\boldsymbol{i}$ kuto-n $]_{P P} \quad a m d a$.

Itja again stove-GEN forehead-LOC.ADV sit.down.3SG

'Itja setzt sich wieder vor den Ofen.'

(KKN_1971_Itja_flk_52, S: Upper Ket')

saj

saj wird verwendet, um die lokale Relation 'in der Mitte' auszudrücken. Diese Konstruktion ist nur in südselkupischen Texten eines Sprechers belegt, wie in Beispiel (73). Das relationale Nomen saj - 'Auge' ist mit einem lokalen Adverbialkasus -gan markiert, ein Allomorph von -qin.

(73) Tab-i-m ant [t'ör-o-n saj-gant $]_{\mathrm{PP}}$ meza-l-gu-t

3SG-EP-ACC boat lake-EP-GEN eye-LOC.3SG pull-INCH-ITER-3SG.O

'Das Boot zog ihn in die Mitte des Sees.'

(PMP_1961_Fairytale_flk_109, S: Middle Ob)

aq

$a q$ - 'Mund' kann verwendet werden, um die lokale Relation 'vor' auszudrücken. Allerdings ist Beispiel (74) das einzige im Korpus überhaupt. Eine zuverlässige Aussage über die Verwendung der Adposition lässt sich daher nicht treffen.

(74)
Qo-lči-mba-tə,
[mada-n aq-ə-t $]_{\mathrm{PP}}$
qory amda
sight-INT.PF-PST.REP-3SG.O door-GEN mouth-EP-LOC.ADV bear sit.3SG 
'(Er) sieht einen Bären, der vor der Tür sitzt.'

(MNN_1977_VillageKuleevo_nar_13, Z: Narym)

s'ünc'ə

Die ursprüngliche lexikalische Bedeutung von s'ünc’ə ist 'Inneres' bzw. 'Magen'. Hierfür finden sich im Korpus keine Beispiele. Stattdessen wird s'ünc'a mit der Bedeutung 'in' verwendet, wie in (75). Die Form zašibanuy 'er stieß' ist vom russischen zašibat' entlehnt. Im Zentralselkupischen ist kein Beispiel belegt, dort wird für die lokale Relation 'in' das Nomen pužo - 'Rohr, Inneres' verwendet (siehe Beispiel (78a) unten).

(75) Ow-s'e zašiba-nu- $\eta$, nitt'a- $\eta \quad$ [na jam-i-n sün'de- $\boldsymbol{\eta}]_{P P}$ force-INSTR knock-AOR-3SG.S there-ADV this trap-EP-GEN inside-LOC.ADV ippi-ku- $\eta$.

lie-ITER-3SG.S

'Er stieß sich hart, liegt dort in dieser Grube.'

(KKN_1971_Itja_flk_18, S: Middle Ket')

kö

Um die räumlichen Relationen 'an', 'zu' oder 'neben' anzugeben, kann das relationale Nomen $k \ddot{o}$ - 'Seite' verwendet werden. Beispiele für eine adpositionale Verwendung, und nur für diese, finden sich in beiden Dialektgruppen. Der Modifikator ist stets mit dem Genitiv markiert, im Zentralselkupischen mit der Suffix-Variante - $t$, wie in (76a), im Südselkupischen mit der Variante $-n$, wie in (76b). Der Modifikator kann auch ein Pronomen sein, wie in (76c).

a. Korg n'anne-l $\quad \varepsilon d \varepsilon-n d \varepsilon \quad k w \varepsilon n-b a, \quad$ nate- $t$ bear forward-ADJZ village-ILL go.away-PST.REP.3SG there-LOC.ADV [mad-i-t ko-ndit $]_{P P}$ to-mba.

house-EP-GEN side-ILL come-PST.REP.3SG

'Der Bär ging ins nächste Dorf, er kam zu einem Haus.' (ChDN_1983_BearCameIntoVillage_nar_17, Z: Vasjugan)

b. Assi kundo qarə-n putšo-nanni ila-s [to-n

NEG long morning-LOC.ADV beaver-ADES live-PST.3SG lake-GEN

q $\overline{\ddot{o}}-$ zan $]_{P P}$ qarra.

side-LOC crane

'Nicht weit vom Biber lebte am Fluß ein Kranich29.'

(BNN_1971_EyesEars_flk_6, S: Middle Ket')

c. N'äpo omtว-n ninka, na to-ntə, $[t \varepsilon p-\partial-n \quad q \overline{\mathbf{o}}-n t \partial]_{P P}$. dug sit-3SG.S there this lake-ILL 3SG-EP-GEN side-ILL

'Eine Ente setzt sich dorthin, auf diesen See, neben ihn.' (NN_1879_Itja_flk_6, Z: Chaja)

moqə

Die ursprüngliche lexikalische Bedeutung von moqə - 'Rücken' erscheint nur noch

${ }^{29} \operatorname{Im}$ Originaltext erscheint kundoqarən zusammengeschrieben. 
in vereinzelten Beispielen im Korpus wie in (77a). Weit häufiger wird moqa mit der Bedeutung 'hinter' verwendet, innerhalb einer Adpositionalphrase, wie in $(77 \mathrm{~b})$ und (Z7c). Der Modifikator (in der Regel erscheint er im Genitiv) kann ein Nomen wie in (77b) oder ein Personalpronomen wie in $77 \mathrm{c}$ sein.

(77)

a. Putšo teb-a-m obr'owno-la-nda pat'-o-l-gwa-t, onda beaver 3SG-EP-ACC beam-PL-ILL put-EP-INCH-ITER-3SG.O himself moqqo-yonda pen-gwa-t. back-3SG.LOC put-ITER-3SG.O

'Der Biber legte sie auf Balken, nahm (die Balken) auf seinen Rücken.' (BNN_1971_EyesEars_flk_11, S: Middle Ket')

b. A na nač'a-t [šoyor-n moyo-n $]_{P P}$ and this there-LOC.ADV stove-GEN back-LOC.ADV qotq-le-l'ča. cough-INCH-INT.PF.3SG

'Und dieser begann dort hinter dem Ofen zu husten.' (SAA_1971_ThreeSisters_flk_83, Z: Narym)

c. Ütč'ega [tep-a-n moqqo-n $]_{P P}$ üt'i-mba. child 3SG-EP-GEN back-LOC.ADV send-PST.REP.3SG

'Das Kind lief ihr hinterher.' (KKN_1971_WhiteHeadedWoman_flk_20, S: Middle Ket')

\section{pužo}

Das Nomen puร̌o hat die ursprüngliche Bedeutung 'Rohr, Inneres'. Im Gegensatz zu s'ünc'a, das die gleiche Bedeutung hat, stammt es aus einem anderen semantischen Feld. Im Korpus finden sich ausschließlich Beispiele in der Verwendung als Adposition 'in' wie in (78a) und (78b). Im Südselkupischen werden beide Varianten verwendet, im Zentralselkupischen ist nur pužo belegt. Mögliche Unterschiede lassen sich aufgrund der geringen Anzahl an Beispielen nicht ausmachen.
a. Ugon ir
wargi-mba
[made-t puzo-git $]_{P P}$ matur. earlier long.ago live-PST.REP.3SG taiga-GEN inside-LOC hero
'Vor langer Zeit lebte im Wald ein Held.'
(ChDN_1983_HeroesDaughter_flk_1, Z: Vasjugan)
b. Tab nenn'a-m-d [sači-t puzo-nd]
3SG sister-ACC-3SG sliver-GEN inside-ILL sage-l-ba-t kunda-n.
stick.through-INCH-PST.REP-3SG.O long-ADV
'Sie steckte ihre Schwester weit in das Kardenband.'
(TMR_1981_Robbers_flk_29, S: Middle Ob)

par

Das relationale Nomen par wird in den Beispielen ebenfalls nicht mehr mit der ursprünglichen lexikalischen Bedeutung 'Oberseite' verwendet. Sämtliche Beispiele aus 
dem Zentral- und Südselkupischen verwenden es mit der Bedeutung 'auf', wie in (79a) und (79b). Bis auf vereinzelte Ausnahmen ist der Modifikator immer mit dem Genitiv markiert.

(79) a. Puja-lzi-ga aj [halz-E-t par-o-nd $]_{P P}$ omdi-ga. owl-DIM-DIM again stump-EP-GEN top-EP-ILL sit-AOR.3SG

'Die Eule saß wieder auf dem Stumpf.'

(ChDN_1983_Pora_flk_32, Z: Vasjugan)

b. Čanže-le kuan-n, [lembe-t par-o-nd] $]_{P P}$ mede-k. go.out-CVB go.away-AOR.3SG eagle-GEN top-EP-ILL achieve-3SG.O

'Er ging hinaus, kam auf den Adler.'

(NN_1855_Hero1_song_214-216, Z/S: Tym/Middle Ob)

tōp

Das Nomen tōp - 'Rand' wird verwendet, um die lokale Relation 'am' auszudrücken. Beispiele sind in beiden Dialektgruppen belegt, wie in (80a) und (80b). Das zentralselkupische Beispiel enthält den Genitiv auf - $t$ (Kolde- $t$ ), im südselkupischen wird das Genitivsuffixe $-n(k \dot{i}-n)$ verwendet.

a. Čāža Iwānuška [Kolde-t tom-mut $]_{P P}$ ji qo-nže-r-ni-d go.3sG Ivanushka Ob-GEN border-PROL and sight-IPFV-FRQ-AOR-3sG.O wary qwel pičā.

big fish pike

'Iwanuschka ging am Rand des (Flusses) Ob (entlang) und erblickte einen großen Hecht.' (TTD_1964_Frog_flk_99, Z: Tym)

b. Tāwa-ka ili-kku-s [ki-n top-qin $]_{P P}$. mouse-DIM live-ITER-PST.3SG river-GEN border-LOC 'Das Mäuschen lebte am Rand des Flusses.' (KMS_1963_HazelgrouseMouse_flk_8, S: Middle Ket')

tibə

Im Südselkupischen wird das Nomen čibe - 'Ende' praktisch ausschließlich in Genitivkonstruktionen mit Lokalkasus verwendet, wie in (81b) und (81c). Im Zentralselkupischen Subkorpus sind lediglich zwei Beispiele überhaupt belegt, eines ist in (81a) gezeigt.

a. $[\bar{E} d \partial-t \quad \text { čibe-yat }]_{P P}$ šunžebal mat. village-GEN end-LOC empty house

'Am Ende des Dorfes ist ein leeres Haus.'

(MNN_1977_VillageKuleevo_nar_3, Z: Narym)

b. Tāmi [t'ör-o-n tīpe-nt $]_{P P}$ uzo man köčku-la-y. upper lake-EP-GEN end-ILL later 1SG go-OPT-1SG.S

'An das obere Ende des Sees werde ich später fahren.' (PMP_1967_AboutItja_flk_20, S: Middle Ob) 
c. To [nu-n čibe-nd $]_{P P}$ maľ̆ kond-ut.

there sky-GEN end-ILL forever sleep-1PL

'Wir schlafen dort bis in alle Ewigkeit (wrtl.: Ende des Himmels).'

(NN_1855_Hero3_song_313, Z/S: Tym/Middle Ob)

\section{ilə}

Das Relationale Nomen ìla 'Boden' kann als Postposition 'unter' verwendet werden, wie im südselkupischen Beispiel (82). Zentralselkupische Beispiele sind im Korpus nicht belegt, dort wird vorrangig das lokale Adverb mit der Bedeutung 'hinunter' verwendet.
Kalabokka kaba pau-nd-se
[kopta-n ülo-nt $]_{P P}$ atte-da
Kalabokka little knife-OBL.3SG-INSTR bed-GEN bottom-ILL hide-IPFV.3sG
'Kalabokka versteckt sich unter dem Bett mit einem kleinen Messer.'
(AGS_1968_FairytaleSnake_flk_61, S: Middle Ket')

Komplexe Postpositionen im Selkupischen haben sich innerhalb eines Grammatikalisierungsprozesses (RELATIONALES NOMEN - > KOPF EINER GENITIV-NP - > ADPOSITION - $>$ KASUSSUFFIX) aus relationalen Nomen entwickelt. Die letzte Stufe des Grammatikalisierungsprozesses - die Entwicklung eines Kasussuffixes - lässt sich im Textkorpus nicht belegen. Die relationen Nomen entstammen unterschiedlichen semantischen Feldern. Aus dem Bereich der Körperteile leiten sich die Postpositionen oli - 'Kopf', qāt - 'Stirn', saj - 'Auge', aq - 'Mund', s'ünc'ə - 'Magen, Inneres', kö - 'Seite, Rippe' und moqə - 'Rücken' her. Aus unterschiedlichen räumlichen Umgebungen entstammen die Postpositionen tōp - 'Rand', par - 'Oberseite', tibə - 'Ende', ìla - 'Boden' und puక̌o - 'Rohr, Inneres'. Mit ihnen können unterschiedliche lokale Relationen ausgedrückt werden. Im Gegensatz zu einfachen Postpositionen sind sie immer mit einem (adverbialen) Lokalkasus markiert.

\subsubsection{Präpositionen}

Ursprünglich kennt das Selkupische keine eigenen Präpositionen. Unter russischem Einfluss finden sich jedoch verschiedene Präpositionen im Textkorpus. Die Beispiele (83a) - (83c) zeigen die aus dem Russischen entlehnte Präposition čeres - '(hin)über, nach'. Dabei wird diese einmal, wie im Russischen auch, als Präposition verwendet, mit nachgestelltem Genitiv-Modifikator, wie in (83a). In (83b) und (83c) hingegen wird die russische Präposition in das selkupische Muster eingefügt und erscheint als Postposition mit pränominalem Genitiv-Modifikator.

$$
\text { a. Tab-i-m nom naša-kin ü̈'a-nži-t: tap qu-la-m }
$$

3SG-EP-ACC god there-LOC.ADV let.go-FUT-3SG.o 3SG person-PL-ACC

[t'eres t'ör-n] $]_{\text {PreP }} p u-t-k u-\check{s}$.

across lake-GEN cross-TR-ITER-IMP.2SG.S

'Gott wird ihn (von) dort gehenlassen: (wenn) er die Menschen über den See rudert.'

(PMP_1961_Fairytale_flk_198, S: Middle Ob) 
b. Qoroyo-t $[k \dot{t}-t \quad \text { č'eres }]_{\text {PreP }}$ olej-sa, a ija-t bear-3SG river-GEN across swim-PST.3sG but son-3sG

ki-t par-to qala.

steep.bank-GEN top-ILL stay.3sG

'Die Bärin schwamm über den Fluss, aber ihr Sohn bleibt am Flussufer.'

(PAV_NN_HowIBearCatch_nar_6, Z: Tym)

c. [T'ör-o-n t'eres $]_{\text {PreP }}$ übə- $n, \quad$ t'ör-o-n kä-n

lake-EP-GEN across set.off-3sG.s lake-EP-GEN steep.bank-GEN

bar-t medi-di- $\eta$.

top-LOC.ADV achieve-IPFV-3SG.S

'Er setzt über den See, erreicht das Steilufer des Sees.'

(PMP_1961_Fairytale_flk_293, S: Middle Ob)

Eine weitere russische Präpostion erscheint in (84a). Hier ist die Präposition nicht entlehnt, sondern wird innerhalb eines Codeswitchings verwendet. Eine ähnliche Konstruktion findet sich im zentralselkupischen Beispiel (84b). Hier wird die russische Präposition $n a$ - 'über' innerhalb des Codeswitching verwendet.

a. Qār tēl āldiga ìde-ne čēnča: İde, taptēl poqqo-n-t morning day grandmother Itja-DAT say.3SG Itja today net-ACC-3SG

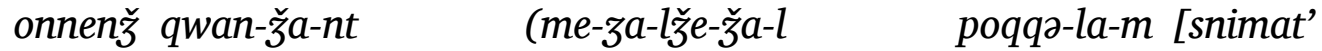
himself go.away-FUT-2sG.s (do-IPFV-TR-FUT-2sG.o net-PL-ACC pick s $\quad$ setej] $]_{\text {Codeswitching). }}$. with net.SG.INSTR)

'Am anderen Tag sagt die Großmutter zu Itja: Itja, du gehst heute selbst die Netze holen (du wirst das Netz machen, das Netz einholen).' (TFF_1967_ItjaAldigaScale_flk_8, S: Middle Ob) 30

b. Nǚ̌̌-p mat paร̌a-le-špi-ha-p Koldi-t tact, grass-ACC 1SG chop-INCH-IPFV-AOR-1SG.O Ob-GEN over kiba-n-jur-get ([na malenəkom $]_{\text {Codeswitching }}$ small-GEN-?-LOC (on small.PRÄPOSITIV sor-u).

low.area.covered.by.water-?)

'Ich mähe das Heu über dem Ob, auf einem kleinen, niedrigen, mit Wasser bedeckten Gelände.'

(TMN_1994_Cow_nar_9, Z: Narym)

\section{Zusammenfassung}

Innerhalb einer Adpositionalphrase können im Selkupischen einfache und komplexe Postpositionen realisiert werden. Einfache Postpositionen sind indeklinabel und

\footnotetext{
${ }^{30} \mathrm{im}$ Original ist qārtēl zusammengeschrieben.
} 
erscheinen in der Regel mit einem pränominalen Modifikator im Genitiv. Komplexe Postpositionen haben sich im Zuge eines Grammatikalisierungsprozesses aus relationalen Nomen entwickelt. Sie werden zum Ausdruck unterschiedlicher semantischer Relationen verwendet. Während die einfachen Postpositionen neben lokalen Relationen auch temporale, modale, kausale und komparative Relationen ausdrücken können, haben die komplexen Postpositionen lediglich lokale Funktion. Aus dem Russischen sind teilweise Präpositionen entlehnt worden, die als Präposition oder Postposition jeweils mit Genitiv-Modifikator verwendet werden. Tabelle 12 fasst sämtliche im Textkorpus belegte Adpositionen zusammen.

Tab. 12: Postpositionen im Selkupischen

\begin{tabular}{|c|c|c|c|c|}
\hline \multicolumn{2}{|c|}{ Einfache Postpositionen } & \multicolumn{3}{|c|}{ Komplexe Postpositionen } \\
\hline $\begin{array}{l}\text { čan } \\
\text { miyin } \\
\text { opti } \\
\text { tare } \\
\text { t'at/taet } \\
\text { t'ombi/ร̌omb } \\
\text { ugōn }\end{array}$ & $\begin{array}{l}\text { 'bis' } \\
\text { 'bei' } \\
\text { 'mit' } \\
\text { 'wie' } \\
\text { 'anstatt, über' } \\
\text { 'während, entlang' } \\
\text { '(da)vor' }\end{array}$ & $\begin{array}{l}\text { tōp } \\
\text { par } \\
\text { tibə } \\
\text { pužo } \\
\text { s'ünc'ə } \\
\text { ila } \\
\text { moqə } \\
\text { kö } \\
q \bar{a} t \\
\text { olì } \\
\text { aq } \\
\text { saj }\end{array}$ & $\begin{array}{l}\text { bei, an } \\
\text { auf } \\
\text { bei, am Rand von } \\
\text { unter, in, hinein } \\
\text { in, hinein } \\
\text { unter } \\
\text { hinter } \\
\text { neben, nach } \\
\text { vor } \\
\text { vor, in (hinein) } \\
\text { in } \\
\text { in der Mitte von }\end{array}$ & $\begin{array}{l}>\text { 'Ecke' } \\
>\text { 'Oberseite' } \\
>\text { 'Ende' } \\
>\text { 'Rohr, Inneres' } \\
>\text { 'Magen, Inneres' } \\
>\text { 'Boden' } \\
>\text { 'Rücken' } \\
>\text { 'Seite, Rippe' } \\
>\text { 'Stirn' } \\
>\text { 'Kopf' } \\
>\text { 'Mund' } \\
>\text { 'Auge' }\end{array}$ \\
\hline
\end{tabular}




\subsection{Verbphrase}

Die Verbphrase (abgekürzt 'VP') im Zentral- und Südselkupischen enthält mindestens eine finite Verbform als Kopf. Zusätzlich kann der Kopf durch ein Adverb, ein Präverb, eine Negationspartikel und verschiedene infinite Verbformen (Infinitiv, Konverb) modifiziert werden. Die finite Verbform ist in der Regel nachgestellt und flektiert nach Tempus und/oder Modus, Numerus und Person. Es kann zwischen subjektiver und objektiver Konjugation unterschieden werden. Eine reflexive Konjugation gibt es nicht. Zwischen Subjekt und finiter Verbform herrscht Kongruenz.

Verben lassen sich in lexikalische (einschl. der Kopula $\bar{e}$ - 'sein') und grammatische Verben unterteilen. Zu den grammatischen Verben gehören die Negationsverben čajki - 'NEG.EX' und tad'a - 'nicht.können' sowie die aus dem Russischen entlehnte Negationspartikel n'etu, die teilweise verbalisiert verwendet wird. Das Modalverb kigə - 'wollen' gehört ebenfalls zu den grammatischen Verben. Des Weiteren gibt es entlehnte Konstruktionen, um Modalität auszudrücken; hierzu zählen die Modalpartikeln nado - 'nötig' (aus dem Russischen entlehnt) und keregey - 'nötig' (aus den Turksprachen entlehnt). Beide Formen werden, wie in den Ursprungssprachen, mit dem Infinitiv eines lexikalischen Verbs verwendet, nado 31 erscheint sowohl im Zentral- als auch im Südselkupischen, keregej ${ }^{32}$ kommt ausschließlich in südselkupischen Texten eines Sprechers vor. In einem Heldenlied finden sich Beispiele, in denen nado als lexikalisches Verb verwendet wird, wie in Beispiel (89b) zu sehen ist. Tabelle 13 zeigt die verschiedenen Varianten von Verbphrasen, die im Zentral- und Südselkupischen vorkommen können, die Tabelle zeigt auch, ob die Modifikatoren prä- oder postverbal erscheinen.

Enthält die VP nur ein Verb, ist dieses Verb ein lexikalisches, die Kopula oder eines der grammatischen Verben ${ }^{33}$, Die Beispielsätze (86a) - (89b) zeigen unmodifizierte Verbphrasen. In Beispiel (86a) enthält die VP die finite Form des lexikalischen Verbs am - 'essen'. In beiden Dialektgruppen kommen VPs vor, die lediglich eine finite Form des Modalverbs kiga - 'wollen' beinhalten, wie die Beispiele (86b) und (86c) zeigen. Die große Mehrzahl der Textbeispiele zeigt kiga jedoch in Kombination mit einer infiniten Verbform.

${ }^{31}$ Praktisch alle Beispiele enthalten eine Nullkopula, deren Präsens durch ihr Fehlen ausgedrückt wird. Lediglich im Zentralselkupischen gibt es ein Beispiel mit overter Kopula und einer AoristMarkierung.

${ }^{32}$ Es gibt ausschließlich Beispiele mit Nullkopula, ob die Kopula im Nich-Präsens overt ausgedrückt wird, läßt sich daher nicht sagen.

${ }^{33}$ Eine Ausnahme bildet das Negationsverb tad'a 'nicht.können', das nur zusammen mit einer infiniten Form erscheint, jedoch lediglich in einem südselkupischen Beispiel belegt ist:

(85) A piri-m watti-gu ted'a-l-bi-t.

but stature-ACC lift-INF cannot-INCH-HAB-3SG.O

'Aber er kann nicht aufstehen (wrtl.: Er kann seine Gestalt nicht hochheben).'

(TMR_1981_AboutItja_flk_25, S: Middle Ob) 
Tab. 13: Muster der selkupischen Verbphrase

\begin{tabular}{|c|c|c|c|c|}
\hline \multirow[t]{2}{*}{ Modifikator } & & Finite Verbform & & Modifikator \\
\hline & & $\begin{array}{l}\text { Lexikalisch } \\
\text { Modalverb } \\
\text { Kopula } \\
\text { NEG.EX }\end{array}$ & & \\
\hline $\begin{array}{l}\text { Adverb } \\
\text { Präverb } \\
\text { Negationspartikel } \\
\text { Negationspartikel } \\
\text { Negationspartikel } \\
\text { Lex. Verb } \\
\text { Lex. Verb } \\
\text { INF } \\
\text { Konverb }\end{array}$ & $\begin{array}{l}+ \\
+ \\
+ \\
+ \\
+ \\
+ \\
+ \\
+ \\
+\end{array}$ & $\begin{array}{l}\text { Lexikalisch } \\
\text { Lexikalisch } \\
\text { Lexikalisch } \\
\text { Lexikalisch } \\
\text { Modalverb } \\
\text { Kopula } \\
\text { Modalverb } \\
\text { Lexikalisch } \\
\text { Lexikalisch }\end{array}$ & + & Adverb \\
\hline & + & Lexikalisch & + & Konverb \\
\hline Lexikalisch $_{\mathrm{INF}}$ & + & Kopula $_{\mathrm{AOR} / \mathrm{PST}}$ & + & nado/ keregey \\
\hline
\end{tabular}

(86)
a. Tawa-kka am-gu-zi-t n'ūžว-m.

mouse-DIM eat-ITER-PST-3SG.O grass-ACC

'Das Mäuschen isst Gras.'

(KMS_1966_MouseGray_flk_5, S: Middle Ket')

b. Kaj tat, ara-ľ̌i-ga, kiga-nd.

what 2sG old.man-DIM-DIM want-2sG.S

'Was, Großvater, willst du?'

(MNS_NN_BullSon_flk_71, Z: Narym)

c. Nejdey as qika-y.

woman NEG want-3sG.S

'Die Frau wollte nicht.'

(PMP_1961_ForestWoman_flk_209, S: Middle Ob)

Der verbale Kopf in den Beispielen (87a) bis (87c) ist eine finite Form des Kopulaverbs. Typischerweise werden mit der Kopula nonverbale Prädikate (87a), Lokativsätze (87b) und Existentialsätze (87c) gebildet. Sie sind in Kapitel (4) näher beschrieben. Die Kopula kann dabei lexikalisch overt ausgedrückt sein, aber auch kovert erscheinen, wie in (88a) und (88b).
a. M’i üče-ga
$\bar{e}$-ya-ut.

1PL child-DIM be-AOR-1PL

'Wir waren Kinder.'

(ChDN_1983_MistressOfFire_flk_1, Z: Vasjugan) 


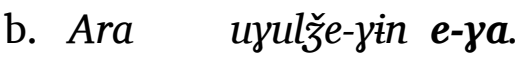

old.man home-LOC be-AOR.3SG

'Der alte Mann war zu Hause.'

(ChDN_1983_MotherInLaw_nar_19, Z: Vasjugan)

c. Tab-i-nnan $\bar{\varepsilon}$-za okkir $n \bar{\varepsilon}-t$.

3SG-EP-ADE be-PST.3SG one daughter-3SG

'Sie hatte eine Tochter.'

(TMR_1981_Devil_flk_2, S: Middle Ob)

(88)

a. Nat'e-yit wiril haj-gidi-l ära.

there-LOC.ADV harmful eye-CAR-ADJZ old.man

'Dort (ist) ein böser einäugiger alter Mann.'

(ChDN_1983_Nikita_flk_12, Z: Vasjugan)

b. Mat taw-t'ä-qindi, $\quad$ na $\bar{\varepsilon} d \varepsilon$-qindi.

1SG this-INDEF-ABL.3SG this village-ABL.3SG

'Ich (bin) von hier, aus diesem Dorf.'

(TMR_1981_Devil_flk_37, S: Middle Ob)

Die aus dem Russischen entlehnte Negationspartikel n'etu erscheint in (89a) verbalisiert als Kopf der VP. Beispiel (89b) zeigt eine weitere russische Entlehnung: Das russische Modalwort nado - 'nötig' wird ebenfalls verbalisiert und erscheint als Kopf der VP. Beispiele dieser Art finden sich nur vereinzelt im Ket'-Subdialekt. Beispiel (89c) zeigt eine VP, die eine finite Form des negativen Existenzialverbs čajki enthält.

(89) a. Komdc-to netu-k.

money-INDEF NEG.EX-3SG.O

'Es gibt kein Geld.'

(MNS_NN_BullSon_flk_5, Z: Narym)

b. Man asa nada-r-a-p.

1SG NEG necessary-VBLZ-EP-1SG.O

'Ich brauche (sie) nicht.'

(NN_1855_Hero1_song_304, Z/S: Tym/Middle Ob)

c. Madur e-k ali čayu-a-n.

hero be-3SG.S or NEG.EX-AOR-3SG.S

' $(\mathrm{Ob})$ es einen Helden gibt oder nicht.'

(NN_1855_Hero1_song_336, Z/S: Tym/Middle Ob)

\subsubsection{Modifikatoren}

Der verbale Kopf kann mit verschiedenen Modifikatoren erweitert werden. Zu den Modifikatoren gehören neben Adverben und Präverben auch Negationspartikeln und verschiedene infinite Verbformen. Lexikalische Verben können mit allen Modifikatoren erweitert werden, die Kopula als finite Komponente erscheint nur in Kombination 
mit Negationspartikeln bzw. den eingangs beschriebenen Partikeln netu und nado. Modalverben können mit Negationspartikeln und Infinitiven modifiziert werden. Die folgenden Abschnitte beschreiben die einzelnen Modifikatoren detaillierter.

\subsubsection{Präverb}

Präverben erscheinen, wie die Bezeichnung bereits impliziert, praktisch ausschließlich präverbal ${ }^{34}$. Im Textkorpus sind für das Südselkupische $t \bar{e}-$ 'weg', für das Zentralselkupische te - 'weg', tak - 'weg' sowie mal - 'geteilt' belegt. Die Beispiele (90a) - (90c) zeigen Präverben als Modifikator aus dem zentralselkupischen Tym- bzw. Vasjugan-Dialekt. In (90d) ist der Modifikator in einem südselkupischen Beispiel gezeigt.

$$
\begin{aligned}
& \text { a. Tabə ājdi-l pai-ye nanš'i-m-d tä } \\
& \text { 3SG sharp-ADJZ knife-INSTR stomach-ACC-3SG away } \\
& \text { qarre-ž'i-l-de. } \\
& \text { cut-INT.PF-INCH-3SG.O }
\end{aligned}
$$

'Er schneidet mit einem scharfen Messer seinen Bauch auf.' (JIF_1968_Kamacha_flk_41, Z: Tym)

b. Haj-gidit-l ära tab-i-p tak čekki-mba-d, eye-CAR-ADJZ old.man 3SG-EP-ACC away unbind-PST.REP-3SG.O $a m-b a-d$. eat-PST.REP-3SG.O

'Der blinde Alte band ihn los, aß ihn.' (ChDN_1983_Nikita_flk_39, Z: Vasjugan)

c. Na šunde-ka-li-ka čwečE par-o-ute mal this bird-DIM-DIM-DIM earth top-EP-PROL separately kwenni-mba-d. carry.away-PST.REP-3SG.O

'Dieser kleine Vogel trug sie (die Nachricht) über das ganze Land.' (ChDN_1983_HerosDaughter_flk_30, Z: Vasjugan)

d. ... na qwel poyqo-qanto tēe i-yə-t. this fish net-ABL.3SG away take-AOR-3SG.O 'Diesen Fisch nahm (er) aus dem Netz heraus.' (NN_1879_Itja_flk_46, S: Chaja)

\subsubsection{Adverb}

Das Selkupische verfügt über eine Vielzahl von Adverben, mit denen Verbphrasen modifiziert werden können. Dabei können Adverben präverbal wie in (91a) und (91c) oder postverbal wie in (91b) und (91d) auftreten, wobei im Belegkorpus die präverbale Verwendung überwiegt.

\footnotetext{
${ }^{34}$ Es gibt wenige Belege, in denen das Präverb postverbal verwendet wird.
} 
a. Tab tahi-še ček koja-ku-mba, 3SG ski-INSTR fast go-ITER-PST.REP.3SG

'Sie lief so schnell Ski, ...' (ChDN_1983_HerosDaughter_flk_15, Z: Vasjugan)

b. Mat kaw-l'ika-t qam̌̆-le-be čwesse.

1SG blood-DIM-3SG pour-INCH-1SG.o backward

'Ich schütte die Blutstropfen zurück.'

(MNS_1984_DaughterOfEarth_flk_15, Z: Narym)

c. Pone čanža-n.

outward go.out-3SG.S

'(Er) geht hinaus.'

(NN_1855_Hero1_song_108, Z/S: Tym/Middle Ob)

d. Man-nan miga-m al'd'a clle.

1SG-ADE neelde-ACC fall.3sG down

'Meine Nadel fällt herunter.'

(TMR_1981_Robbers_flk_13, S: Middle Ob)

\subsubsection{Infinite Verbformen}

Das Selkupische verwendet eine Reihe analytischer Verbalkonstruktionen, deren infinite Komponente in der Regel ein Konverb oder der Infinitiv eines lexikalischen Verbs ist und der finiten Verbform vorangeht. Konverben erscheinen nur mit lexikalischen Verben 35 . Infinitive erscheinen mit lexikalischen Verben und dem Modalverb kiga - 'wollen'. Nur in den südselkupischen Ket'-Dialekten ist zusätzlich das Modalverb $\bar{\varepsilon} s s u$ 'sich sehnen, verlangen, wollen 36' belegt.

In (92a) und (93) ist die finite Form ein grammatisches Verb, hier das Modalverb kiga - 'wollen' in der dialektalen Variante qiga. Die infinite Verbform ist der Infinitiv des lexikalischen Verbs ora - 'halten' bzw. poža - 'schneiden'.
a. Ko-l'čā-det
nadè-li-ka-p
i qigā-det ora-l-gu. sight-INT.PF-3PL girl-DIM-DIM-ACC and want-3PL hold-INCH-INF

'Sie sehen das Mädchen und wollen es festhalten.' (TTD_1964_WildDucks_flk_47, Z: Tym)

b. P'arge-m-t kìga-k mat poža-lži-gu.

stomach-ACC-3SG want-1SG.S 1SG knife-INT.PF-INF

'Den Bauch möchte ich aufschneiden.'

(ILP_1981_ItjaPoenegesse_flk_10, S: Middle Ob)

\footnotetext{
${ }^{35}$ Für Konverben, die in Auxiliarverbindungen verwendet werden, ist die finite Verbform lexikalisch unterschiedlich stark desemantisiert. Es stellt sich die Frage, ob es sich dort noch um ein lexikalische oder bereits ein grammatisches Verb handelt, vgl. auch das Exkurs-Kapitel 6 für eine detaillierte Beschreibung konverbaler komplexer Prädikate

${ }^{36}$ Bykonja (2005: 312) übersetzt die Form mit 'захотеть' (zaxotet') - 'wollen' ins Russische.
} 
In Beispiel (93) wird das Modalverb $\bar{\varepsilon} s s u$ - 'sich sehnen, verlangen, wollen' (in der dialektalen Variante $\bar{e} z u$ ) in einer Inifinitivkonstruktion verwendet. Eine derartige Konstruktion ist nur im südselkupischen Ket'-Dialekt belegt. Helimski (1998: 575) beschreibt eine ähnliche analytische Verbkonstruktionen für das (Nord) Selkupische (ütïr-qo esïmpa-k - 'drink-INF become-1SG.S - > Ich bin durstig37.'). Im Zentralselkupischen findet sich kein Beispiel mit dem Modalverb $\bar{\varepsilon} s s u$.

Qar'i-min wassi-l'ewl'e qwan-gu ēzu-y.

morning-PROL get.up-CVB go.away-INF want-3sG.S

'Am Morgen stand er auf und wollte wieder losgehen.'

(KMS_1966_TwoSisters_flk_57, S: Middle Ket')

Die Verbphrase kann zwei lexikalische Verbformen (VerbFINIT + Infinitiv) enthalten, wie in (94a) und (94b). In der Regel geht die finite Verbform dem Infinitiv voraus (94a). In beiden Dialektgruppen finden sich jedoch auch Beispiele, in denen die umgekehrte Reihenfolge verwendet wird, wie in (94b), Unterschiede zwischen den Dialekten lassen sich nicht ausmachen.
a. Wot paja
qwan-n
ära-p
p'ē-gu.
look.here old.woman go.away-AOR.3sG old.man-ACC search-INF
'Sieh, die alte Frau geht los, um den alten Mann zu suchen.'
(SAA_1971_MoleWifeVar_flk_19, Z: Narym)
b. Tep n'üža-m patt'-a-l-gu qwen-ni.
3SG grass-ACC put-EP-INCH-INF go.away-AOR.3SG
'Er geht Gras mähen.'
(KKN_1971_IvanAndGod_flk_5, S: Upper Ket')

Ist die infinite Verbform in der Verbphrase ein Konverbial, können adverbiale Funktionen sowie komplexe Handlungsverläufe (auch mit temporalem Bezug) ausgedrückt werden. Die Beispiele (96a) und (96c) zeigen Verbphrasen, in denen die Konverbiale adverbiale Funktion übernehmen. Erscheint das Konverbial der finiten Verbform nachgestellt, wie perle - 'gebratenes' in (96a) und okkirlä - 'für immer' in (96b), können Depiktivsätze 38 geformt werden. Beispiel (96c) zeigt die für das Südselkupische typische Wortfolge Konverb + infinite Verbform.

\footnotetext{
${ }^{37}$ Die Konstruktion ließe sich problemlos mit dem Modalverb 'wollen' übersetzen, ohne den Sinn von Helimskis Übersetzung zu verlieren. Im Nordselkupischen hat das Verb esï die zweite Bedeutung 'werden'.

${ }^{38}$ Neben Konverben können mit Hilfe des Translativs depiktive Funktionen ausgedrückt werden, wie in Beispiel (95).
}

(95) Kalabokka [qāla p’al-gālìn].

Kalabokka stay.3SG friend-CAR

'Kalabokka bleibt allein (wrtl.: ohne Freund).'

(TFF_1967_KalabokkaPönegessa_flk_9, S: Middle Ob) 
a. M’̇ šep-ka-p am-ga-ut per-le.

1PL chipmunk-DIM-ACC eat-AOR-1PL fry-CVB

'Wir aßen das Erdhörnchen gebraten.'

(SAG_1984_StoryAboutLife_nar_28, Z: Narym)

b. Pönegessä tö-l'a-pe-št tö-nža i

Pönege come-CVB-?-2sG.ACC come-FUT.3sG and qwan-ne-ň̆a-št okkir-lä.

catch-DRV-FUT.3SG-2SG.ACC one-CVB

'Pönegesse kommt zu dir und fängt dich für immer.'

(TFF_1967_ItjaAldigaPönegesse_flk_19, S: Middle Ob)

c. Kud=nej qwesse ili-l'e ass para-l-ba-n.

who $=\mathrm{EMPH}$ back live-CVB NEG return-INCH-PST.REP-3SG.S

'Niemand ist lebend zurückgekehrt.'

(PMP_1961_Fairytale_flk_132, S: Middle Ob)

Konverbkonstruktionen, mit denen komplexe Handlungen ausgedrückt werden, sind in den Beispielen 97a bis 99 dargestellt. Für eine detaillierte Betrachtung komplexer konverbaler Prädikate sei hier auf das Exkurs-Kapitel 6 verwiesen, in dem nicht nur die verschiedenen Gruppen konverbaler Prädikate ausführlich beschrieben und mit Beispielen belegt sind, sondern zusätzlich auch das Nordselkupische betrachtet wird.

Die in (97a) und (97b) gezeigten Verbphrasen, deren infiniter Teil ein Konverb und deren finiter Teil eines der Phasenverben ist, kommen in beiden Dialektgruppen vor, im Südselkupischen sogar praktisch ausnahmslos mit Konverben. Als Phasenverben kommen oldə - 'beginnen', kwatta - 'beginnen', übə - 'beginnen' sowie mančə (Zentralselkupisch) bzw. malčə (Südselkupisch) - 'beenden' vor. Im Zentralselkupischen wird typischerweise eine Konstruktion mit Infinitiv verwendet, wie in (97b).

a. Pōne-la-n pal'd'u-l'e oldi- $\boldsymbol{\eta}$.

outside-PL-LOC.ADV go-CVB begin-3SG.S

'Er begann, hinauszugehen 39 .'

(PVD_1961_FarmAssault_flk_145, S: Upper Ob)

b. Kuša-göt tat lèm-a-p mē-gu kuöttā-lža-l, to when-LOC.ADV 2SG board-EP-ACC do-INF begin-INT.PF-2SG.O then tärbe-š manžö-gu kaj-l-žön $\bar{e}-m a$.

think-IMP.2SG.S look-INF thing-ADJZ-PP be-COND.3SG

'Wenn du ein Brett zu machen beginnst, dann denke (daran), zu schauen, von welchem Stamm es sei40.'

(KAO_1912_SmartPeople_trans_8, Z: Tym)

\footnotetext{
${ }^{39}$ Die Analyse des Suffixes -la als Plural in pōne-la- $n$ ist problematisch. Möglicherweise liegt ein Schreibfehler vor und das Suffix kann eher als Lokativ (-gan) interpretiert werden.

${ }^{40}$ Die Form kaj-l-žön lässt sich nicht eindeutig analysieren. Das Suffix -žön ist möglicherweise eine lokale Adposition, sie erscheint in anderen Texten im Korpus ebenfalls in Adpositionalphrasen.
} 
Mit Hilfe von Bewegungsverben können ebenfalls komplexe Handlungsabläufe ausgedrückt werden, wie in (98), indem ein Bewegungsverb (das Konverb) die Art der Bewegung t'itta - 'schreiten't1 ausdrückt, das zweite Bewegungsverb (die finite Verbform) den Pfad čă ̌̌a - 'gehen'42. Die semantische Bedeutung der finiten Verbform ist in derartigen Konstruktion teilweise desemantisiert, in der Form, als dass hier lediglich eine einzige Bewegung stattfindet und nicht zwei.

(98) I İd'e t'itta-r-lä čāz̆a.

and Itja walk-FRQ-CVB go.3sG

'Und Itja schreitet.'

(TFF_1967_IdjaAldigaNet_flk_5, S: Middle Ob)

Einen höheren Grad an semantischer Reduktion findet sich in Auxiliarkonstruktionen, wie in Beispiel (99). Die finite Verbform āmdā - 'sitzen' erscheint hier nicht als Vollverb, sondern wie ein Hilfsverb, das vorrangig Träger grammatischer Informationen wie Tempus, Modus und Person ist. Erhalten bleibt ein Bedeutungsanteil, den Schönig (1984: 50) als „sekundäre Implikation“ bezeichnet. Diese sekundären Implikationen beinhalten „räumlich-richtungsmäßige“ Implikationen bei Verben, die zur „Gruppe der Eigenbewegung, des Etwas-Bewegens und der Nichtbewegung gehören." (ebd.: 50). Die Konverben artšabbale 'drink.CVB und apstabbale 'eat.cVB bilden die semantische Komponente der Konstruktion. Sie können darüber hinaus mit unterschiedlichen Derivationssuffixen markiert werden.

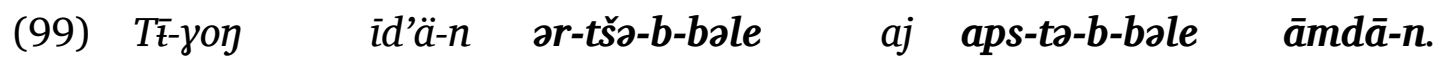

Tatar-prince Itja-GEN drink-TR-HAB-CVB and foot-TR-HAB-CVB sit-3sG.S

'Der Tatarenprinz füttert Itja und gibt ihm zu Trinken.'

(NN_1913_Itja_flk_42, S: Middle Ob)

Das Selkupische kennt ursprünglich keine analytisch gebildeten Tempusformen. Verbphrasen mit drei Verbformen kommen daher in der Regel nicht vor. Beispiel (100a) zeigt eine aus dem Russischen entlehnte Konstruktion, bestehend aus der Modalpartikel nado - 'nötig', der Kopula $\bar{e}$ - 'sein' und der infiniten Form ade - 'warten'. Es ist das einzige Beispiel dieser Art im Korpus. Häufiger kommen Konstruktionen wie in (100b) vor, in denen nado mit Nullkopula auftritt.

a. Ade-lž-gu nāde $\bar{e}-h a \quad$ maš̀tk.

wait-INT.PF-INF be.necessary be-AOR.3SG 1SG.ACC

'Er sollte auf mich warten.'

(TTD_1964_Frog_flk_77, Z: Tym)

b. Tadim qwat-ku nādo era-u-m.

now kill-INF be.necessary husband-1sG-ACC

'Jetzt muss man meinen Mann töten.'

(PVD_1961_FarmAssault_flk_147, S: Chaja)

\footnotetext{
${ }^{41}$ Anstelle von beispielsweise 'laufen', 'kriechen' oder ähnliches.

${ }^{42}$ Anstelle beispielsweise ‘hinausgehen', ‘ankommen', ‘eintreten’ oder Ähnlichem.
} 


\section{Phrasentypen}

Eine alternative Konstruktion zu nado erscheint nur im Südselkupischen. Hier wird die aus Turksprachen entlehnte Modalpartikel keregen - 'nötig' in der Variante $k$ 'er'eg'en, analog zu nado, ausschließlich mit Nullkopula, verwendet, wie in (101). Die Lesart mit covertem Subjekt in der 2. Person ergibt sich aus dem Kontext. Dir wörtliche Übersetzung lautet: 'Sie muss man nehmen.'

(101) Tab-i-m jī-gu k'er'eg'ey.

3SG-EP-ACC take-INF necessary

'Sie musst du nehmen.'

(PMP_1961_ForestWoman_flk_181, S: Middle Ob)

\subsection{Negierte Verbphrase}

Lexikalische Verben, Modalverben und die Kopula können mit Hilfe von Negationspartikeln modifiziert werden. Konverben können im Selkupischen nicht negiert werden. Innerhalb einer negierten Verbphrase geht die Negationspartikel dem Kopf (in dem Fall der (finiten) Verbform) unmittelbar voraus. $\mathrm{Zu}$ den verwendeten Negationspartikeln gehören ašša, aš nordselkupisch, aza, aha, aya, $\bar{a}$, jā (zentralselkupisch), assi, assa, as(a), aza, $\bar{a}$ (südselkupisch) sowie die imperativische Negationspartikel ik $\dot{i}$ für alle Dialektgruppen und die aus dem Russischen entlehnte Partikel n'e, die lediglich in Konstruktionen mit russischer Syntax in zentralen und südlichen Texten verwendet wird wie in den Beispielen (104a) - (104d).

Neben den Negationspartikeln verfügt das Selkupische über ein negatives Existenzi-

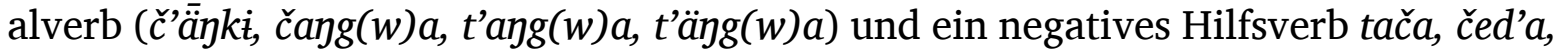
ted'a - 'nicht können', die beide nicht zum Ausdruck der Standardnegation verwendet werden. Nur in zentralen Dialekten wird darüber hinaus die aus dem Russischen entlehnte Negationspartikel n'etu als negatives Existenzialverb verwendet.

Tabelle 14 zeigt die im Korpus belegten Negationswörter in den drei Dialektgruppen. Die Beispiele (102a) und (102b) zeigen negierte transitive Verbphrasen, die Beispiele

Tab. 14: Negationswörter im Selkupischen

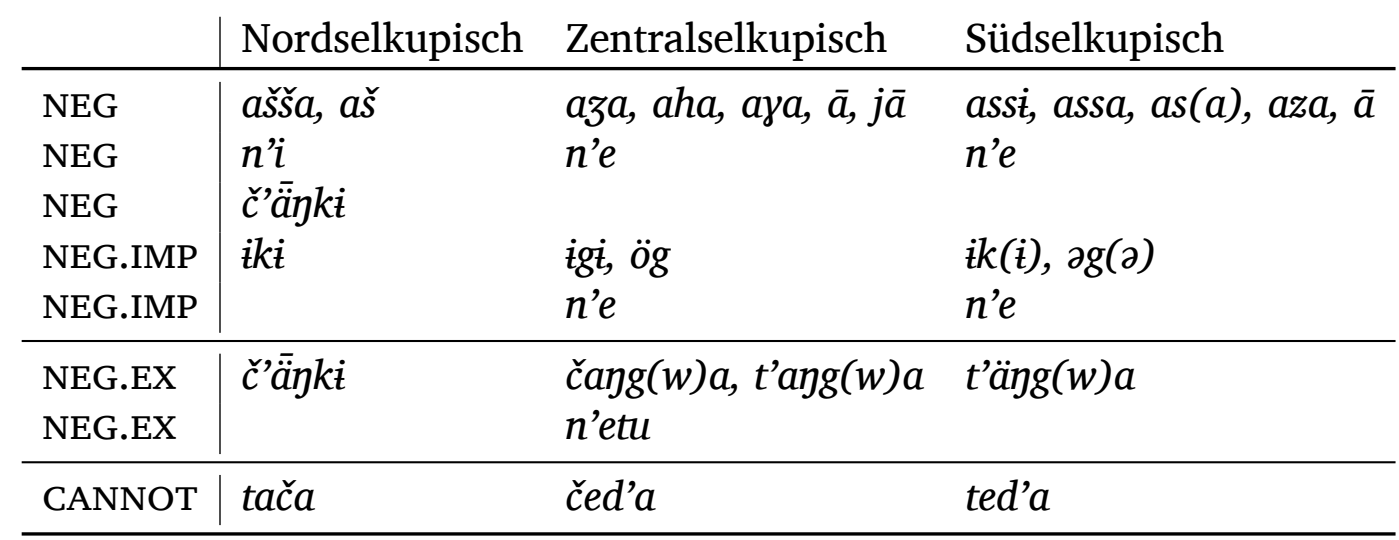


(102c) und (102d) negierte intransitive Verbphrasen. Der einzige formale Unterscheid zWischen affirmativer und negierter Phrase ist somit die Negationspartikel.

(102) a. [Ǎ̌ üte-nta-p] $]_{V P \text {. }}$

NEG let.go-FUT-1SG.O

'Ich lasse ihn nicht los.'

(AVA_1973_Ichakicha2_flk_27, N: Taz)

b. Tab-i-t pe-mba-dit, pe-mba-dit, [aga

3SG-EP-PL look.forPST.REP-3PL look.for-PST.REP-3PL NEG

ko-mba-dit $]_{V P}$.

sight-PST.REP-3PL

'Sie suchten und suchten ihn, sahen ihn nicht.'

(ChDN_1983_Pora_flk_20, Z: Vasjugan)

c. $[\text { Aza qwan-za-k }]_{V P}$.

NEG go.away-FUT-1sG.S

'Ich werde nicht gehen.'

(TMR_1981_SapSapiha_flk_7, s: Middle Ob)

d. Paja-la-t [as wesa-q-wa-t $]_{V P}$.

woman-PL-3SG NEG get.up-ITER-AOR-3PL

'Ihre Frauen standen nicht auf.'

(KKN_1971_FiveCarpBrothers_flk_80, S: Upper Ket')

Die Negationspartikel $i k \dot{t}$ wird ausschließlich in imperativen Phrasen und Sätzen verwendet. Im Textkorpus ist sie lediglich in Verbphrasen belegt, wie in den Beispielsätzen (103a) - (103d).

a. Tat nami $[\mathbf{i} k \mathbf{i} \text { tom-tit }]_{V P}$.

2SG this NEG.IMP say-2SG.O

'Sag das nicht!'

(BVP_1973_East_flk_37, N: Taz)

b. Tan man nodik [igi twi-l'-ed] $]_{V P}$ !

2SG 1SG sterlet NEG.IMP steal-INCH-IMP.2SG.O

'Du stiel nicht meinen Stör!'

(ChDN_1983_Nikita_flk_27, Z: Vasjugan)

c. Tat $\dot{\text { iy lari-mb-le-nd } \quad k u d i-m=n a j a . ~}$

2SG NEG.IMP be.afraid-HAB-OPT-2sG.S who-ACC = NEG.EMPH

'Fürchte dich vor niemandem!'

(TTD_1964_CalfHare_flk_23, Z: Tym)

d. Mašep [ik t'āč-k-l'e-l] $]_{V P}$ !

1SG.ACC NEG.IMP shoot-ITER-OPT-2SG.O

'Erschieß mich nicht!'

(SEV_1980_HazelGrouse_flk_7, S: Middle Ob) 


\section{Phrasentypen}

Die aus dem Russischen entlehnte Negationspartikel n'e ist nur in zentralen und südlichen Dialekten in Verbphrasen belegt. Sämtliche Belege zeigen Konstruktionen mit russischer Syntax wie in den Beispielen (104a) - (104d): n'e tuzi (<russ. tuzit'), n'e mozet < russ. moč'), n'e mogu (< russ. moč') bzw. n'e popal bi (< russ. popast'). Nordselkupische Beispiele sind nicht belegt.

a. N'e tuzi, carēwič!

NEG be.sad.IMP son.of.the.tsar

'Sei nicht traurig, Zahrensöhnchen!'

(TTD_1964_Frog_flk_33, Z: Tym)

b. [...] meder-gu n'e mozet.

[] reach-INF NEG can.3SG

'... sie kann (sie) nicht erreichen.'

(TTD_1964_WildDucks_flk_12, Z: Tym)

c. $[\text { N'e } \mathrm{mogu}]_{V P}, \quad\left[\mathbf{N}^{\prime} \boldsymbol{e} \text { mogu }\right]_{V P}$.

NEG CAN.1SG.PRS NEG CAN.1SG.PRS

'Ich kann nicht, ich kann nicht.' (PVD_1961_FarmAssault_flk_129, S: Chaja)

d. [...] što struška-la qə-n sun'e-ddə [n'e popal] $]_{V P}$ bï?

[] that shaving-PL river-GEN inside-ILL NEG hit.PST.3SG if

'..., dass die Hobelspäne nicht in den Fluss geworfen werden?' (PVD_1961_FarmAssault_flk_25, S: Chaja)

Das negative Existenzialverb č ‘̈̈jki bzw. seine Varianten formen negierte Existenzialsätze. In allen Dialektgruppen sind Beispiele belegt wie in (105a) - (105c).

(105) a. N'än'-mit nōti [čäyka] $]_{V P}$

bread-1PL then NEG.EX.3SG

'Wir haben kein Brot.'

(ALA_1977_ThreeBrothers_flk_145, N: Taz)

b. Mì-nan nin qozar [t'äng-wa] $]_{V P}$.

1PL-ADE here mammoth NEG.EX-CO.3SG

'Bei uns gibt es keine Mammuts.'

(KFN_1967_Mammoth_flk_1, Z: Tym/Narym)

c. Tab-la-nan el'mād-la [t'ǟu-za-t $]_{V P}$.

3SG-PL-ADE child-PL NEG.EX-PST.3SG

'Sie hatten keine Kinder.'

(SEV_1980_HazelGrouse_flk_2, S: Middle Ob)

Nur im Zentralselkupischen wird zusätzlich die aus dem Russischen entlehnte Negatiopspartikel $n$ 'etu als negatives Existentialverb verwendet, wie die Beispiele (106a) und (106b) zeigen. 
a. [...] or-ma [n'etu $]_{V P 1}$, qaj-o-m

force-NOM/ACC.1SG NEG.EX.3SG what-EP-NOM/ACC.1SG

[n'etu $]_{V P 2}$.

NEG.EX.3SG

'Ich habe keine Kraft, ich habe nichts.'

(SAA_1971_MoleWife_flk_28, Z: Narym)

b. Tab-nan or-t [n'etu-pa]

3SG-ADE force-3SG NEG.EX-PST.REP.3SG

'Er hatte keine Kraft.'

(ChDN_1984_DaughterOfEarth_flk_13, Z: Narym)

Das negative Hilfsverb tača mit den Variapten čed'a und ted'a ist vor allem in nordselkupischen Texten belegt wie in Beispiel (107a). Belege finden sich vereinzelt aber auch in zentralen und südlichen Dialekten wie in (107b) und (107c). Sein affirmatives Pendant ist das Verb teni - 'können, wissen', in nördlichen Dialekten auch mit der Variante tenimi. Das negative Hilfsverb folgt typischerweise dem Hauptverb, das in allen Korpusbelegen der Infinitiv ist. Die von Wagner-Nagy (2015: 34f.) beschriebene Verwendung eines Supinums anstelle des Infinitivs lässt sich im Korpus nicht belegen.
a. Qälit-t ponä tanti-qo [tača-l-no:-titt $]_{V P}$.
Nenets-PL outward(s) go.out-INF cannot-INCH-CO-3PL
'Die Nenzen können nicht auf die Straße heraus.'
(KMP_1971_NyomalPorky_flk_34, N: Middle Taz)
b. Tab-it-t tü̈-p čadi-gu [čed'a-l-ba-dit $]_{V P}$.
3SG-EP-PL fire-ACC light-INF cannot-INCH-DUR-3PL
'Sie können kein Feuer anzünden.'
(ChDN_1983_MistressOfFire_flk_38, Z: Vasjugan)
c. A piri-m watti-gu [ted'a-l-bi-t $]_{V P}$,
rok-wat-pa.
but stature-ACC lift-INF cannot-INCH-PST.REP-3SG.O
stick-DRV-PST.REP.3SG
'Er kam nicht los, er blieb kleben.'
(TMR_1981_AboutItja_flk_34, S: Middle Ob)

\section{Zusammenfassung}

Insgesamt ist also festzuhalten, dass die selkupische Verbphrase zwischen einer und drei Verbformen enthalten kann, wobei drei Verbformen nur mit Hilfe entlehnter Partikeln (nado und keregey) realisiert werden können. Enthält die VP nur eine (finite) Verbform, kann diese entweder eine lexikalische Verbform, das Modalverb kiga bzw. das nur in den Ket'-Dialekten des Südselkupischen belegte $\bar{\varepsilon} s s u$ 'sich sehnen, verlangen, wollen', die Kopula $\bar{e}$ - 'sein' oder ein negatives Existentialverb (čạki் - 'NEG.EX') 
bzw. das negative Hilfsverb (tad'a - 'nicht.können') sein. Die finite Form eines lexikalischen Verbs oder des Modalverbs können mit infiniten Verbformen wie dem Infinitiv oder einem Konverb modifiziert werden.

Mit Hilfe von Negationspartikeln (vgl. Tabelle 14) können sowohl transitive als auch intransitive Verben negiert werden. Die Negationspartikel geht dabei dem Kopf unmittelbar voraus wie in den Beispielen (102a) - (102d). Die Negationspartikel iki formt in allen Dialektgruppen imperativische Ausdrücke wie in den Beispielen (103a) - (103d). Nur in den zentralen und südlichen Dialekten wird auch die aus dem Russischen entlehnte Negationspartikel n'e verwendet, um imperativische Ausdrücke zu bilden wie in den Beispielen (104a) - (104d). Das Selkupische verfügt über ein negatives Existentialverb, das in allen drei Dialektgruppen verwendet wird wie in den Beispielen (105a) - (105c). Zusätzlich wird nur in zentralen Dialekten die aus dem Russischen entlehnte Negationspartikel n'etu zur Bildung von negierten Existentialsätzen verwendet (Beispiele (106a) und (106b)). Das negative Hilfsverb tača bzw. seine Varianten kann in allen Dialektgruppen verwendet werden (Beispiele (107a) (107c). 


\section{Syntax von Sätzen}

Bei der Betrachtung von Sätzen im Selkupischen ist es zweckmäßig, einfache Sätze vor komplexen Sätzen zu erörtern. Erstere enthalten ein vollständiges Prädikat sowie seine Argumente, Komplemente und/oder Adjunkte. Letztere bestehen aus mehreren einfachen Sätzen, die miteinander verbunden sind. Aus diesem Grund werden einfache Sätze in Kapitel 4, komplexe Sätze in Kapitel 5 beschrieben. Einen Rahmen bilden die grammatischen Relationen und die Struktur der Satzkonstituenten, die im folgenden Abschnitt 3.1 erläutert werden sowie die Abfolge der Konstituenten auf der Satzebene in Abschnitt 3.2.

\subsection{Satzglieder und grammatische Relationen}

Wie bereits oben angesprochen, ist das Selkupische eine NominativAkkusativsprache, in der das overte Subjekt intransitiver Sätze (S) in der Regel ebenso markiert ist, wie das Subjekt transitiver Sätze (A $\left.A^{1}\right)$ - mit dem Nominativ. Belebte Subjekte werden nicht von unbelebten unterschieden. Subjekt und Prädikat kongruieren in Person und Numerus. Das Patiens transitiver Sätze (P) ist in der Regel mit dem Akkusativ markiert. Die Beispiele (108a) und (108b) enthalten intransitive Verben (menc - 'jagen' und pat - 'hinlegen'). Ihre Argumente sind das Personalpronomen $t a b$ - '3sG' in (108a) und das emphatische Pronomen onž - 'selbst' in (108b). Beide Subjekte erscheinen unmarkiert, also im Nominativ. Das Agens in (108c) ist ebenfalls das unmarkierte Personalpronomen tab - '3SG'. Das Patiens (das direkt Objekt) čobir 'Beere' erscheint im Akkusativ.

(108) a. Tab-i-n wesa menc-r-ba-dit. 3SG-EP-PL all hunt-FRQ-PST.REP-3PL

'Sie jagten alle.'

(ChDN_1983_Pora_flk_7, Z: Vasjugan)

b. Onz naj natt'ā-t pat-pa,

himself also there-LOC.ADV go.down-PST.REP.3SG

qonda-l-ba.

sleep-ICH-PST.REP.3SG

'Sie selbst legte sich auch dorthin, schlief ein.'

(TMR_1981_Robbers_flk_31, S: Middle Ob)

${ }^{1}$ Die Abkürzungen S und A stehen für die Argumente intransitiver Verben bzw. das Agens transitiver Verben. 
c. Tab čobir-p awē-špa-s

3SG berry-ACC eat-IPFV-PST.3SG

'Er aß Beeren.'

(SEV_1981_SisterBrother_flk_24, S: Middle Ob)

\subsubsection{Subjekt}

Das grammatische Subjekt erscheint in der Regel satzinitial, Interrogativpronomen oder Adverbien können ihm aber vorausgehen. Zumeist wird das Subjekt durch ein Pronomen wie in den obigen Beispielen (108a) - (108c) oder mittels einfacher bzw. komplexer Nominalphrasen ausgedrückt wie in (109a) und (109b).

a.

$\begin{array}{lll}{[\text { Anda }]_{N P}} & \text { porolža-nde čanni-mba, onže } \\ \text { boat stream.course-ILL go.out-PST.REP.3SG himself }\end{array}$

kur-e-špe-llit-mba.

run-EP-IPFV-INCH-PST.REP.3SG

'Ein Boot fuhr in die Stromschnelle, es fuhr selbst.'

(ChDN_1983_Nikita_flk_15, Z: Vasjugan)

b. [Tä sai-n pija-j äta-j qorra $]_{N P}$ ti-le qwässa-n-d four eye-GEN owl-ADJZ reindeer-ADJZ stallion fly-CVB iron-GEN-3SG ollo-nd swängi-r-i-ldä- $\eta$.

head-ILL stick.through-FRQ-EP-INT.PF-3SG.S

'Ein vieräugiger Eulen-Rentier-Hengst kam geflogen, steckte sich in das Schaberköpchen hinein.'

(SVG_1964_IitekaPineweldju_flk_7, S: Upper Ket')

Selten werden Numerale und Demonstrativpronomen als Subjekt verwendet. In diesen kopflosen Phrasen trägt das Numeral bzw. das Demonstrativpronomen die Numerusmarkierung, wie die Pluralmarkierung in (110c). Die grammatischen Subjekte in (110a) und (110b) stehen (unmarkiert) im Singular.

a. Okkir kwes-sa

$$
\text { uši-gu ču-t par-o-nd, a peže-p }
$$

one go.away-PST.3SG work-INF earth-GEN top-EP-ILL but axe-ACC

hugulže-git aulži-mba-t.

home-LOC.ADV forget-PST.REP-3SG.O

'Einer ging hinaus, um in der Erde zu arbeiten, aber er vergaß die Axt zu Hause.'

(SVD_2002_StrongSelkup_flk_2, Narym)

b. Te taddi-n amda-lta, a me sadayun qwa-žaj

2PL here-ADV sit-IMP.2PL, but 1PL two-LOC go.away-1DU

'Ihr sitzt hier, aber wir gehen zu zweit.' (PVD_1961_FarmAssault_flk_33, S: Chaja)

c. Nā-la kit'-wat-ple kur-a-nna-t.

this-PL get.frightened-DETR-CVB run-EP-AOR-3SG.O 
'Diese erschraken und rannten weg.'

(SEV_1981_SisterBrother_flk_49, S: Middle Ob)

Etwa zwei Drittel (64\%) aller Subjekte im Textkorpus sind Pronomen, wobei nur $17 \%$ von ihnen overt ausgedrückt werden. Da das Selkupische eine Pro-Drop-Sprache ist, sind pronominale Subjekte nur selten overt realisiert, wie in den Beispielen (108a) - (108c) oben. Typischwerweise wird auf sie mittels der Verbalendung verwiesen, sie erscheinen also covert. In den Beispielen (111a) - (113c) sind alle pronominalen Subjekte nicht lexikalisch ausgedrückt.

(111) Erste Person
a. K'ēmdì-ze sārì-kka-m.
black.cherry-INSTR bind-ITER-1SG.O
'(Ich) umwickele (ihn) mit einer Traubenkirschenrute.' (TMR_1967_Poenege_flk_29, S: Middle Ob)
b. Čeli-t tabe-tču-gu kwaja-ka-j.
day-LOC.ADV squirrel-TR-INF go-ITER-1DU
'Am Tag gehen (wir (zwei)) Eichhörnchen jagen.'
(ChDN_1983_MotherInLaw_nar_11, Z: Vasjugan)
c. Üde-r-le kuatt-ut.
drink-FRQ-CVB begin-1PL
'(Wir) fingen an, zu trinken.'
(NN_1855_Hero2_song_122, Z/S: Tym/Middle Ob)

(112) Zweite Person
a. $k \bar{u} \quad \check{c} \bar{a} \breve{z} \varepsilon-n d ?$
'Wohin gehst (du)?'
(ChDN_1983_Nikita_flk_85, Z: Vasjugan)
b. kai-tko sep tada-r-e-li?
what-TRL 1SG.ACC bring-FRQ-EP-2DU
'Warum führt (ihr (zwei)) mich (fort)?'
(NN_1855_Hero1_song_55, Z/S: Tym/Middle Ob)
c. Qaj-kō-na nač'e-do šêr-yu-mba-lt.
'Warum seid (ihr) dort hineingegangen?'
(SDP_1964_FairytaleBlackZar_flk_51, Z: Narym)
what-TRL-this there-INDEF enter-ITER-PST.REP-2PL

(113) Dritte Person
a. Mat šier-n.
house.ILL enter-3SG.S
'(Er) trat in das Haus.'
(NN_1855_Hero2_song_267, Z/S: Tym/Middle Ob) 
b. Kuča-si-di oqqo-mizan.

go.to.sleep-PST-3DU one-near

'(Sie (zwei)) legten sich zusammen schlafen.'

(BNN_1971_DogSearchedMistress_flk_8, S: Middle Ket')

c. Našša-kit pönege-t kap-t-i-p tan n'epka-lla-de.

there-LOC.ADV Pönege-GEN blood-PL-EP-ACC away suck-OPT-3PL

'Dann schlürfen (sie) dort Pöneges Blut.'

(ChDN_1983_ItjasTown_flk_11, Z: Vasjugan)

\subsubsection{Direktes Objekt}

Das direkte Objekt in einem Satz wird typischerweise mit einer Nominalphrase ausgedrückt, möglich sind aber auch Adjektive, Partizipien oder Pronomen, die als Objekt fungieren. Unabhängig von der lexikalischen Realisierung eines Subjektes wird das direkte Objekt in der Regel mit dem Akkusativ markiert wie in den Beispielen (114a) und (114b) mit nominalem Objekt und (115a) und (115b) mit pronominalem Objekt.

(114) Nominalphrase

a. Tab čobir-p awē-špa-s

3SG berry-ACC eat-IPFV-PST.3SG

'Er aß Beeren.'

(SEV_1981_SisterBrother_flk_24, S: Middle Ob)

b. Teaga-p kere-lže-d.

sword-ACC wave-TR-3SG.O

'(Er) schwenkte das Schwert.'

(NN_1855_Hero2_song_162, Z/S: Tym/Middle Ob)

(115) Pronomen

a. Mat šindi pō-ye aw-di-mba-k.

1SG 2SG.ACC wood-INSTR eat-TR-PST.REP-3SG.O

'Ich habe dich mit Holz gefüttert.'

(ChDN_1983_MistressOfFire_flk_19, Z: Vasjugan)

b. Omdi-ľ̆i-mba-q-t

ando-nd tab-d-i-p.

sit.down-INT.PF-PST.REP-?-3PL boat-ILL 3SG-PL-EP-ACC

'(Sie) setzten sie in das Boot.'

(MNS_1984_BrotherSister_flk_67, Z: Narym)

Neben Objekten, die mit dem Akkusativ markiert sind, gibt es im Korpus Beispiele, in denen das direkte Objekt einer monotransitiven Konstruktion in der unmarkierten Form erscheint, dem Nominativ. Beispiele dieser Art finden sich sowohl in zentralen als auch südlichen Dialekten. Die ersten Versuche, Regeln für die Verwendung unmarkierter Objekte im Selkupischen aufzustellen, gehen auf Castrén (1854: 140; 309) und Prokof'ev (1931: 85-86) zurück und sind in Wickman (1955) beschrieben 
worden. Die Form des direkten Objektes im (Nord) Selkupischen ist auch in einem Artikel von Wagner-Nagy/Szeverényi (2013) untersucht worden. In ihrem Artikel untersucht Wegener (2018) die differentielle Objektmarkierung (DOM) im Südselkupischen. Ausgehend von den Parametern, die von Sinnemäki (2014) genannt werden, nimmt sie an, dass lediglich die Informationsstruktur einen Einfluss auf die DOM im Zentral- und Südselkupischen haben kann. Wegener schließt die anderen Parameter wie Tempus, Modus, Animatheit, Definitheit, Verwandtschaftsterminologie und Eigennamen aus. Bis heute ist die Funktion dieser unmarkierten Objekte allerdings nicht endgültig geklärt. Auch in dieser Arbeit fehlt der Raum, Regeln für die Verwendung unmarkierter Objekte im Zentral- und Südselkupischen zu untersuchen. Die Beispiele (116a) - (118b) geben einen Überblick über die Umgebungen, in denen unmarkierte Objekte verwendet werden (können). In (116a) und (116b) erscheinen einfache Nominalphrasen (tabek - 'Eichhörnchen', šêpka - 'Erdhörnchen', kur 'Hermelin' und $p \bar{o}$ - 'Holz' (hier in der Bedeutung von 'Mörser') als unmarkierte Objekte.

a. Qwad-e-špa-d'i tabek, šēpka, kur.

kill-EP-IPFV-3DU.o squirrel chipmunk stoat

'Sie jagten Eichhörnchen, Erdhörnchen, Hermeline.

(SAI_1984_StoryAboutLife_nar_3, Z: Narym)

b. A paja-t āwo-t somma-j pō i-ndo-t.

and old.woman-3sG mother-3sG mortar-ADJz tree take-INFER-3sG.o

'Und die alte Mutter-Frau nahm den hölzernen Mörser.2.'

(TFF_1967_KolobokkaPönegessa_flk_36, S: Middle Ob)

Nominale Objekte, die mit Numeralen modifiziert sind, erscheinen in der Objektposition in beiden Dialektgruppen in den meisten Fällen im Nominativ, wie in (117a) und (117b). Daneben gibt es jedoch auch mit Akkusativ markierte Objekte nach Numeralen, wie in (117c) und (117d), ebenfalls in beiden Dialektgruppen. Dabei zeigen die Beispiele keine Unterschiede bezüglich der Numerale.
a. ... čel-d
kwat-k-ut
[köt tawa], kobi čolgimba.
day-LOC.ADV catch-ITER-1PL ten mouse skin shine-PST.REP.3SG
'Am Tag fingen wir zehn Mäuse, (ihr) Fell glänzte.'
(SAI_1984_StoryAboutLifeLong_nar_37, Z: Narym)
b. I [nakur tut] qwätti-mba
and three crucian kill-EP-PST.REP.3SG
'Und er fing drei Karauschen.'
(TFF_1967_ItjaAldigaScale_flk_17, s: Middle Ob)
c. T'el-e-di kwedi-mba,
[nagur čuru-m]
day-3sG be.beautiful-PST.REP.3SG three sand-ACC

\footnotetext{
${ }^{2}$ Im Original ist sommajpō zusammengeschrieben.
} 
menni-mba- $\gamma$.

pass.through-PST.REP-3DU.S

'Der Tag ist schön, sie passierten vier Ufer.'

(ChDN_1983_ItjaStayedAlone_flk_10, Z: Vasjugan)

d. [Saľ̆e madur-a-p] kuas-sa-p.

seven hero-EP-ACC kill-PST-1SG.O

'Sieben Helden tötete ich.'

(NN_1855_Hero4_song_111, Z/S: Tym/Middle Ob)

Mit dem Quantifizierer koči - 'viele' modifizierte nominale Objekte können in der unmarkierten Form erscheinen. Beispiele finden sich vor allem in den südselkupischen Dialekten wie in (118a), im Zentralselkupischen zeigt lediglich ein Beleg (übersetzter Text) ein unmarkiertes Objekt in (118b), daher lassen sich keine konkreten Aussagen über die Verwendung von Nominativ-Objekten mit Quantifizierern machen.

a. [Kot'ə kual] kual-le übə-r-ə-ddat.

much fish fish-CVB start-FRQ-EP-3PL

'Sie fingen an, viele Fische zu fangen.'

(NN_1913_Itja_flk_87, Chaja)

b. Tab takki-l-ba-t [koče-k kwel].

3SG gather-INCH-PST.REP-3SG.O much-ADV fish

'Er fing viele Fische.'

(KIA_2014_ItjaKet_trans_21, Narym)

Direkte Objekte in Imperativsätzen erscheinen meistens im Nominativ. Diese Eigenschaft teilt das Selkupische mit einigen anderen uralischen Sprachen (für das Nenzische siehe Janhunen (2002), für das Nganasanische siehe Wagner-Nagy (2019)). Im Textkorpus ist auch der Akkusativ als Kasusmarkierung in imperativen Sätzen vorhanden 3 . In südselkupischen Materialien ist das imperative Konjugationsparadigma weitestgehend erhalten, zentralselkupische Texte belegen nur noch den Imperativ Singular in der zweiten und dritten Person. Hinsichtlich der Kasusmarkierung und deren (regelhafter) Verwendung lassen sich anhand des Belegmaterials keine eindeutigen Aussagen treffen. In beiden Dialektgruppen erscheinen in allen vorhandenen Numerus und beiden Konjugationsarten (subjektiv und objektiv) sowohl unmarkierte als auch pronominale oder nominale Akkusativobjekte, wie in (119a) - (120e) exemplarisch gezeigt ist. Eine ausführliche Betrachtung imperativer Konstruktionen erfolgt in Kapitel 4

(119) subjektive Konjugation

a. It'e-ne t'ara- $\eta$ : s'im sappo-zi-q!

Itja-DAT say-3SG.S 1SG.ACC spring.out-DRV-IMP.2sG.S

'Er sagt zu Itja: Zieh mich raus!'

(KKN_1971_Itja_flk_19, S: Upper Ket')

${ }^{3}$ Belegt sind die zweite und dritte Person Singular, die erste, zweite und dritte Person Dual sowie die zweite und dritte Person Plural. 
b. ... tan mey noppì mē-ga-q!

2SG 1SG.DAT glove do-ITER-IMP.2SG.S

'Du mach mir Handschuhe!'

(KMS_1966_TwoSisters_flk_31, S: Middle Ket')

c. Kot’a $\boldsymbol{n} \overline{\boldsymbol{e}}-\boldsymbol{m}$ ko-qqü-ja-k!

much daughter-ACC find-ITER-AOR-IMP.SG.S

'Bekomm viele Töchter!'

(NN_1913_Ija_flk_84, S: Middle Ob)

d. M'izenit tǖ-m m'e-je-š!

1PL.DAT fire-ACC give-AOR-IMP.2SG.S

'Gib uns Feuer!'

(ChDN_1983_MistressOfFire_flk_66, Z: Vasjugan)

(120) objektive Konjugation
a. ... tan ulo-m mannu-mp-laj.
2sG head-ACC look-hab-IMP.1DU
'Lass uns deinen Kopf anschauen!'
(PMP_1961_ForestWoman_flk_62, S: Middle Ob)

b. Peke-ka t'äri- $\eta \quad p \bar{u}-l o \quad$ ki-ke-m nit'a-n hazel.grouse-DIM say-3SG.s cross-IMP.1DU river-DIM-ACC there-ADV pemi-bi-čcot.

hunt-HAB-IPFV-1PL

'Das Haselhuhn sagte: Lass uns den Fluss überqueren, wie jagen dort.' (KKA_NN_HazelGrouse_flk_7, S: Upper Ket')

c. Paja čenča era-ndì-ni: hirì-l nade-m old.woman say.3SG old.man-OBL.3SG-DAT/ALL snow-ADJZ girl-ACC me-laj.

make-IMP.1DU

'Die Alte sagte zum Alten: Lass uns ein Schneemädchen machen!' (TET_1979_SnowGirl_flk_6, S: Middle Ket')

d. Na pēge żža-l-g-wa aze-qind, this hazel.grouze say-INCH-ITER.3SG father-DAT/ALL.3SG

awe-qind: mašep nād-li!

mother-DAT/ALL.3SG 1SG.ACC get.married-IMP.2DU

'Dieses Haselhuhn sagte zu seinem Vater und seiner Mutter: Verheiratet mich!'

(SEV_1980_HazelGrouse_flk_14, S: Middle Ob)

e. ... İt'a-m tĩ tan-na-lot, lōs-a-la!

Itja-ACC here bring-AOR-IMP.2PL devil-PL

'Bringt Itja hierher, Geister!'

(NN_1879_Itja_flk_66, S: Chaja) 


\subsubsection{Indirektes Objekt}

Indirekte Objekte erscheinen als zweite Komplemente ditransitiver Verben und werden mit dem Dativ markiert. Im Selkupischen zählen hierzu neben den physikalischen und mentalen Transferverben auch benefaktive Konstruktionen. Indirekte Objekte sind typischerweise (belebte) Nomen oder Pronomen. Die Beispiele (121a) - (121e) sind exemplarisch, eine detaillierte Betrachtung erfolgt in Kapitel 4 .
a. Man tēgga tamd'el me-ža-u qūdajo-m.
1SG 2SG.DAT tomorrow give-FUT-1SG.O pull.strap-ACC
'Ich werde dir morgen einen Strick geben.' (PVD_1961_FarmAssault_flk_67, S: Chaja)
b. Čel'enga me-xi-d n'oma-n kōwri-p. calf give-PST-3SG.O hare-DAT small.carpet-ACC
'Das Kalb gab dem Hasen den Teppich.' (TTD_1964_CalfHare_flk_29, Z: Tym)
c. NE-l mekka me-d! daughter-2SG 1SG.DAT give-IMP.2SG.O
'Gib mir deine Tochter!' (ChDN_1983_HeroesDaughter_flk_34, Vasjugan)
d. Ku-la-tdi-ne qai-m=nē-s ket-ku-t. person-PL-OBL.3SG-DAT what-ACC $=$ EMPH-CLIT tell-ITER-3SG.O 'Seinen Leuten hat er nichts erzählt.' (PVD_1961_FarmAssault_flk_10, Chaja)
e. Mēga s'ut-ti porək-i-m! 1SG.DAT sew-IMP.2SG.O clothing-EP-ACC 'Näh mir Kleidung!' (PMP_1961_ForestWoman_flk_189, Middle Ob)

\subsubsection{Adjunkte}

Adjunkte sind optionale Ergänzungen eines Satzes. Adverbien, Nominalphrasen mit und ohne Adverbialkasus sowie Postpositionalphrasen werden typischerweise im Selkupischen verwendet, um Lokalität, Richtungsangaben wie Ausgangs- und Endpunkt, Ziele, Wege sowie temporale Relationen auszudrücken. Temporaladverbien stehen in der Regel satzinitial, wie in (122a), Lokaladverbien weisen eine größere Variation hinsichtlich ihrer Stellung im Satz auf. Das Lokaladverb tepa - 'hier' in (122b) steht präverbal, in (122c) erscheint natt'a - 'dort' postverbal und ist mit einem lokalen Adverbialkasus $(-t)$ markiert. Die Nominalphrase ö di - 'Frühling' in (122d) ist ebenfalls mit einem lokalen Adverbialkasus - $t$ markiert. Das relationale Nomen క̌onžo - 'Hälfte' in (122e) ist als Kopf der Nominalphrase mit dem Lokativallomorph -qan markiert. Der Modifikator $p i$ - 'Nacht' erscheint im Genitiv. Eine ähnliche Konstruktion zeigt das 
Beispiel (122f). Die Genitivform des Modifikators madam - 'Haus.GEN' ist untypisch und möglicherweise ein Schreibfehler.
a. Ugot kupes $\bar{e}-k \quad k o ̈ y-e-q i t, \quad$ tabe
earlier merchant be-3sG.S Kenga-EP-LOC 3SG
taw-e-špi-mba-t qozar-n amdi-p.
buy-EP-IPFV-PST.REP-3SG.O mammoth-GEN horn-ACC

'Früher gab es einenKaufmann an der Kjonga, er kaufte Mammuthorn.'

(KFN_1967_Mammoth_flk_4, Z: Tym)

b. Kuarme tan teja konde-k!

brother-in-law 2sG here sleep-IMP.2sG.S

'Schwager, schlaf du hier!'

(NN_1855_Hero1_song_387, Z/S: Tym/Middle Ob)

c. Tö-mba natt'̄a-t, okkir tiditk āmdi-s.

come-PST.REP.3SG there-LOC.ADV one cedar sit-PST.3SG

'(Er) kam dorthin, (wo) eine Zeder wuchs.'

(SEV_1981_SisterBrother_flk_36, S: Middle Ob)

d. $K \dot{\boldsymbol{t}} \quad \overline{\boldsymbol{d}} \boldsymbol{d} \dot{\mathrm{i}} \mathrm{t} \quad q \bar{\partial}-p \quad q e t t-k u-t$,

river spring-LOC.ADV coast-ACC flood-ITER-3SG.O

'Der Fluss überflutet im Frühling das Ufer, ...'

(SAA_1984_MyGrandmother_nar_13, Narym)

e. Pi-n-žonžo-qan na sü tü-ssa-n täp-käni šit' night-GEN-half-LOC this dragon come-PST-3SG.S 3SG-ILL.3SG two ola-zi-se.

head-CRC-INSTR

'Um Mitternacht kam dieser Drache zu ihnen, mit zwei Köpfen.' (AGS_1968_FairytaleSnake_flk_92, Middle Ket')

f. It'e üt'i-mb'i-ku- $\eta \quad$ mad-a-m t'ombi, asse

Itja let.go-HAB-ITER-3SG.S house-EP-GEN along NEG

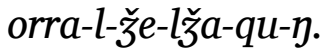

hold-INCH-DRV-INT.PF-ITER-3SG.S

'Itja läuft im Haus umher, es gelingt (Loz) nicht, (ihn) zu fassen.

(KKN_1971_Itja_flk_15, Upper Ket')

\subsection{Wortstellung}

Aus den Beispielen aus Abschnitt 3.1 wird ersichtlich, dass syntaktische Relationen im Zentral- und Südselkupischen mit morphologischen Mitteln wiedergegeben werden. Die Wortstellung spielt für die Wiedergabe syntaktischer Relationen eine untergeordnete Rolle. Helimski (1998: 576) beschreibt die Grundwortstellung im Selkupischen 
als SOV. Diese Grundwortstellung ist charakteristisch auch für die anderen samojedischen und ob-ugrischen Sprachen (vgl. Comrie 1988: 569). Daneben sind auch andere Wortstellungen wie OSV und SVO möglich (Helimski 1998: 576). Die Korpusdaten bestätigen die Angaben teilweise: So lässt sich in den zentralselkupischep Texten recht einheitlich eine Dominanz der Wortstellung SOV beobachten, wie in (123a). Für die südselkupischen Texte ist das Bild deutlich uneinheitlicher: Neben SOV (123b) und SVO (123d) kommen auch OSV (123c) und OVS (123e) vor.

a. qup ki-t par-o-yit ni-nga.

person river-GEN top-EP-LOC stand-AOR.3SG

np.h:S np:Poss np:L

'Ein Mann steht am Ufer.'

(ChDN_191983_GirlAndIce_flk_12, Z: Vasjugan)

b. Irra s'inat ondi-ne qwero-y.

old.man 2SG.PL.ACC himself-DAT call-3SG.S

np.h:S pro.h:O

'Der Alte ruft euch zu sich.'

(KKN_1971_FiveCarpBrothers_flk_46, S: Upper Ket')

c. Tab-ì-p qū-la mādì-r-gu-zōa-dit.

3SG-EP-ACC person-PL ask.for-FRQ-ITER-PST-3PL

pro.h:O np.h:S

'Die Menschen baten sie.'

(ILP_1981_StonyOldWoman_flk_3, S: Middle Ob)

d. Ezzi-t ünga-lzi-mbi-s-t ne-n-t

father-3SG listen-INT.PF-HAB-PST-3SG.O daughter-GEN-3SG

np.h:S

küdo-r-e-m.

dream-DRV-EP-ACC

np.h:O

'Der Vater hörte den Traum der Tochter.'

(PMP_1961_Fairytale_flk_29, S: Middle Ob)

e. P'arge-m-t kiga-k mat poža-ľ̆i-gu.

stomach-ACC-3SG want-1SG.S 1SG unpick-INT.PF-INF

np:O

pro.h:S

'Seinen Bauch möchte ich aufschneiden.'

(ILP_1981_ItjaPönegesse_flk_10, S: Middle Ob) 


\section{Einfacher Satz}

Als syntaktische Einheit auf der Textebene enthält der einfache Satz im Zentral- und Südselkupischen mindestens ein Prädikat. Es lassen sich verschiedene Satzmodi unterscheiden: Deklarativsätze, Imperativsätze und Interrogativsätze. In deklarativen, interrogativen und imperativen Sätzen werden die Kategorien Person, Numerus, Tempus und/oder Modus morphologisch an der finiten Verbform markiert. In Existenzialund Lokativsätzen (die den Deklarativsätzen untergeordnet werden) kann die Kopula in nonverbalen Konstruktionen als Nullkopula auftreten. In allen Satztypen des einfachen Satzes kongruiert die finite Verbform in Numerus und Person mit dem grammatischen Subjekt, das mittels einer Nominalphrase im Nominativ ausgedrückt werden kann. Da das Selkupische eine Pro-Drop-Sprache ist, müssen pronominale Argumente nicht lexikalisch overt ausgedrückt werden, auf sie kann auch allein durch den Kontext verwiesen werden bzw. erfolgt die morphologische Markierung der Person ausschließlich an der finiten Verbform wie in (124).

(124) Suguľ̌e kuačo-nd üda-p.

home city-ILL let.go-1sG.O

'[Ich] ließ [ihn] nach Hause gehen.'

(NN_1855_Hero1_song_189, Z/S: Tym/Middle Ob)

Die nachfolgenden Kapitel geben einen Überblick über transitive und intransitive Deklarativsätze (4.1), Interrogativsätze (4.2) und Imperativsätze (4.3). Im Anschluss an die Beschreibung affirmativer Satztypen folgt die Darstellung der negierten Varianten.

\subsection{Deklarativsatz}

Deklarativsätze kommen im zugrunde liegenden selkupischen Textkorpus am häufigsten vor. Das folgende Kapitel beschreibt intransitive sowie mono- und ditransitive Konstruktionen im Zentral- und Südselkupischen.

\subsubsection{Intransitiver Deklarativsatz}

Das Subjekt eines intransitiven Deklarativsatzes erscheint im Nominativ, wie in (125a) und (125b). Ein (pronominales) Subjekt muß nicht lexikalisch overt ausgedrückt werden. In (125c) wird auf das Subjekt nur mittels der am Verb markierten Personalendung verwiesen. 
a. M’i lak-wa-ča-ut.

1PL laugh-DRV-RFL-1PL

'Wir lachten.'

(SAG_1984_StoryAboutLife_nar_18, Z: Narym)

b. Sap kwesse čāzi-mba.

egret backward go-PST.REP.3SG

'Der Reiher ging zurück.'

(TMR_1981_SapSapiha_flk_8, S: Middle Ob)

c. Laka-ľ̆a-i.

move-INT.PF-1DU

'Wir brachen auf.'

(NN_1855_Hero3_song_40, Z/S: Tym/Middle Ob)

\subsubsection{Transitiver Deklarativsatz}

Ein transitiver Deklarativsatz enthält mindestens ein mono- oder ditransitives Verb. Das Subjekt des Satzes erscheint im Nominativ (vgl. Kapitel 3.1.1) und ist in der Regel das Agens. Das (direkte) Objekt des Satzes kann sowohl mit dem Akkusativ als auch mit dem Nominativ markiert sein (siehe Bekker 1995b: 106, 141) und ist in der Regel das Patiens des Prädikats. Da der Nominativ kein Kasussuffix trägt, ist die Form unmarkiert. Genitivsubjekte, wie sie von Helimski (1998: 576) vor allem für nominalisierte Verbformen beschrieben werden, sind im Korpus nicht belegt.

\subsubsection{Monotransitiver Satz}

Monotransitive Sätze enthalten ein fakultatives oder obligatorisches Objekt. Die Subjekte in den Beispielen (126a) und (126b) sind lexikalisch overt, die Objekte stehen im Akkusativ und sind ebenfalls lexikalisch overt. In beiden Dialektgruppen erscheinen im Textkorpus auch Nominativobjekte, wie pō - 'Holz' in (126c) und tabek - 'Eichhörnchen', šêpka - 'Erdhörnchen' und kur - 'Hermelin' in (126d). Die von Helimski (1998: 576) dargestellten morphosyntaktischen Faktorent, nach denen NominativObjekte ausgewählt werden, lassen sich im Textkorpus nur bedingt belegen. Es finden sich vielmehr in allen Fällen auch Akkusativobjekte wie das indefinite Objekt čobirp - 'Beeren' in (126b) oder direkte Objekte im Imperativ (Abschnitt 4.3 unten), für die es im Textkorpus auch Belege mit Akkusativmarkierung gibt.

a. $[\text { Adja-d }]_{A}$ i-mba-d $\quad[\text { na olo-p }]_{P . A C C}$.

father-3SG take-PST.REP-3SG.O this head-ACC

'Der Vater nahm diesen Kopf.'

(ChDN_1983_Pora_flk_40, Z: Vasjugan)

${ }^{1}$ Der Nominativ wird demnach immer für Objekte verwendet, wenn das Verb im Imperativ steht und meistens, wenn das Objekt indirekt ist. 
b. $[\mathrm{Tab}]_{A}[\check{c o b i r}-p]_{P . A C C} a w \bar{e}-\check{s}-p a-s$.

3SG berry-ACC eat-IPFV-PST.3SG.

'Er aß Beeren.'

(SEV_1981_SisterBrother_flk_33, S: Middle Ob)

c. $A \quad[\text { paja-t }]_{A} \quad \bar{a} w o-t \quad\left[\begin{array}{ll}s o m m a-j & p \bar{o}\end{array}\right]_{P . N O M}$

and old.woman-3SG mother-3SG mortar-ADJZ tree.NOM

i-nda-t.

take-INFER-3SG.O

'Und die alte Mutter-Frau nahm den hölzernen Mörser.2'

(TFF_1967_KolobokkaPönegessa_flk_36, S: Middle Ob)

d. Qwad-e-špa-d'i [tabek $\left.]_{\text {P.NOM }},[\check{s} \bar{e} p k a]_{P . N O M}, k u r\right]_{P . N O M}$.

kill-EP-IPFV-3DU.O squirrel.NOM chipmunk.NOM stoat.NOM

'Sie jagten Eichhörnchen, Erdhörnchen, Hermeline.

(SAI_1984_StoryAboutLife_nar_3, Z: Narym)

Nominale Objekte, die mit Numeralia modifiziert sind, erscheinen in der Objektposition in den meisten Fällen im Nominativ, wie in (127a) und (127b). Daneben lassen sich jedoch auch Akkusativobjekte nach Numeralia beobachten, wie in (127c) und (127d). Die Beispiele zeigen dabei keine Unterschiede hinsichtlich der Numeralia.

(127) a. I [nakur tut $]_{P . N O M}$ qwäti-mba

and three crucian.NOM kill-EP-PST.REP.3SG

'Und er fing drei Karauschen.'

(TFF_1967_ItjaAldigaScale_flk_17, S: Middle Ob)

b. ... čel-d kwat-k-ut [köt tawa $]_{\text {P.NOM, }}$ kobi

day-LOC.ADV catch-ITER-1PL ten mouse.NOM skin

čolgimba.

shine-PST.REP.3SG

'Am Tag fingen wir zehn Mäuse, (ihr) Fell glänzte.'

(SAI_1984_StoryAboutLifeLong_nar_37, Z: Narym)

c. [Saľ̆e madur-a-p] $]_{P . A C C}$ kuas-sa-p.

seven hero-EP-ACC kill-PST-1SG.O

'Sieben Helden tötete ich.'

(NN_1855_Hero4_song_111, Z/S: Tym/Middle Ob)

d. T'ele-di kwedi-mba, [nagur čuru-m] $]_{P . A C C}$

day-3sG be.beautiful-PST.REP.3SG three sand-ACC

menni-mba- $\gamma$.

pass.through-PST.REP-3DU.S

'Der Tag ist schön, sie passieren drei Ufer.'

(ChDN_1983_ItjaStayedAlone_flk_10, Z: Vasjugan)

\footnotetext{
${ }^{2}$ Im Original ist sommajpō zusammengeschrieben.
} 
Auch mit dem Quantifizierer koči - 'viel' modifizierte nominale Objekte können in der unmarkierten Form erscheinen. Beispiele finden sich vor allem in den südselkupischen Dialekten wie in (128a). Im Zentralselkupischen zeigt lediglich ein Beleg (übersetzter Text) ein unmarkiertes Objekt in (128b), daher lassen sich keine genaueren Aussagen über die Verwendung unmarkierter Objekte in Kombination mit Quantifizierern treffen.

a. [kot’ə kual $]_{P . N O M}$ kual-le $\overline{u ̈ b \partial-r-\partial-d d a t . ~}$

much fish.NOM fish-CVB start-FRQ-EP-3PL

'Sie fingen an, viele Fische zu fangen.'

(NN_1913_Itja_flk_87, S: Chaja)

b. $[\mathrm{Tab}]_{A}$ takki-l-ba-t $\quad[k o c ̌ e-k \quad k w \varepsilon l]_{\text {P.NOM. }}$.

3SG gather-INCH-PST.REP-3SG.O much-ADV fish.NOM

'Er fing viele Fische.'

(KIA_2014_ItjaKet_trans_21, Z: Narym)

\subsubsection{Ditransitiver Satz}

Ditransitive Konstruktionen enthalten außer einem Agens (A) zwei obligatorische Objekte: ein direktes Objekt respektive Thema (T) sowie, je nach Verb, einen Rezipienten ( $R$ ) oder Adressaten oder Nutznießer. Im Selkupischen lassen sich verschiedene ditransitive Konstruktionen unterscheiden. Konstruktionen in denen das ditransitive Verb ein physikalisches Transferverb ist, wie mi - 'geben', tati - 'bringen, geben' oder qwande - 'tragen' bilden Konstruktionen, in denen das Agens ein Objekt bzw. Thema (T) veranlasst, in den Besitz eines belebten Rezipienten (R) überzugehen und damit laut Malchukov/Haspelmath/Comrie (2010: 2) als „the most typical three-argument constructions".

Ist das ditransitive Verb ein kognitives oder mentales Transferverb, wie ati - 'zeigen', kät - 'erzählen, sagen' und "̈̈tə - 'schicken', ist der Rezipient ein Adressat der Handlung, die vom Agens ausgeführt wird. Den dritten Typ bilden benefaktive Konstruktionen, in denen der Rezipient Nutznießer ist. Im Folgenden werden die unterschiedlichen ditransitiven Konstruktionen in Beispielen dargestellt.

Bei der nachfolgenden Betrachtung ditransitiver Konstruktionen wird der Fokus auf der Argumentstruktur liegen, indem die formalen Markierungen von Rezipient und Thema beschrieben werden. Die Einordnung in einen theoretischen Rahmen orientiert sich an Malchukov/Haspelmath/Comrie (2010). Die dortige Betrachtung unterscheidet hinsichtlich des Ausrichtungstyps, welches der beiden Objekt-Argumente (R) und $(\mathrm{T})$ sich wie das direkte Objekt (P) eines transitiven Satzes verhält. Für die selkupischen ditransitiven Sätze ist das der indirektive Typ. Das bedeutet, das Thema des ditransitiven Satzes wird genauso markiert wie der Patiens des monotransitiven Satzes. Da im Selkupischen monotransitive Objekte sowohl mit dem Nominativ als auch mit dem Akkusativ markiert werden können (vgl. Kapitel 4.1.2.1 auf Seite 100), 
erscheint auch das Thema ditransitiver Konstruktionen sowohl morphologisch unmarkiert als auch mit dem Akkusativ markiert. Nominale und pronominale Rezipienten werden in den zentralen und südlichen Dialekten identisch markiert.

\section{Physikalische Transferverben}

Zu den physikalischen Transferverben gehören im Selkupischen mi - 'geben', tati 'bringen', qwande - 'tragen' und üto - 'schicken'. Das Thema erscheint entweder im Akkusativ oder unmarkiert im Nominativ (vgl. auch Kapitel 3.1.2).

mi

Das Verb $m i$ - 'geben' gilt als der protypische Vertreter eines physikalischen Transferverbs. In den zentral- und südselkupischen Subkorpora kommen nominale und pronominale Rezipienten vor, die jeweils mit dem Dativsuffix - $n \dot{t}$ - oder einem entsprechenden Allomorph markiert werden. Das Thema kann mit dem Akkusativ kodiert werden, wie in den Beispielen (129a) - (130b).
a. [čel'egga $]_{A} m e-x \dot{i}-d$
$\left[n^{\prime} \text { oma-n }\right]_{R}[k o ̄ w r i-p]_{T}$.
calf give-PST.REP-3SG.O hare-DAT small.carpet-ACC
'Das Kälbchen gibt dem Hasen den Teppich.'
(TTD_1964_CalfHare_flk_29, Z: Tym)
b. $[N a \text { ol-m }]_{T} \quad[\text { nenn'a- } n-d]_{R} \quad t \bar{a} d \dot{t}-m b a-t$.
this head-ACC sister-DAT-3SG bring-PST.REP-3SG.O
'Diesen Kopf brachte er der Schwester.'
(SEV_1981_SisterBrother_flk_86, S: Middle Ob)

a. $[k \bar{c} c ̌ i-m]_{T} \quad[e u-t]_{A} \quad[t a b-n e]_{T} m e-z i-t, \quad$ püsej-la-m nej. earring-ACC mother-3SG 3SG-DAT give-PST-3SG.O bead-PL-ACC also

'Die Mutter gab ihr einen Ohrring und auch Perlen.'

(PMP_1961_ForestWoman_flk_83, S: Middle Ob)

b. $[\mathrm{man}]_{A}[\text { tègga }]_{R}$ tamd'el me-ža-u $[q \bar{u} d \partial \gamma o-m]_{T}$.

1SG 2SG.DAT tomorrow give-FUT-1SG.O pull.strap-ACC

'Morgen werde ich dir einen Strick geben.'

(PVD_1961_FarmAssault_flk_67, S: Chaja)

Die zentral- und südselkupischen Beispiele (131a) - (132b) zeigen unmarkierte Themata, mit nominalem Rezipienten in (131a) und (131b) sowie mit pronominalem Rezipienten in (132a) und (132b). Die Argumente ditransitiver Verben müssen durchaus nicht obligatorisch lexikalisch overt ausgedrückt werden. In (131a) und (131b) wird auf den Agens mittels der Kodierung am verwiesen (- $l$ - '2SG.S', - $t$ - '3SG.O' bzw. $-d$ - 'IMP.2SG.O').
a. $[\check{C} ' \ddot{u} d e]_{T}$ me-le-el
[tab-d-o-n nūčka-la-yend $]_{R}$.
horse.NOM give-OPT-2sG.S 3SG-PL-EP-GEN grandson-PL-DAT.3SG 
'Gib seinen Enkeln Pferde!'

(SDP_1964_FairytaleBlackZar_flk_89, Z: Narym)

b. [Ira-nne $]_{R}$ m'i-ya-t [šitta tuto $]_{T}$.

old.man-DAT give-AOR-3SG.O two crucian.NOM

'Er gab dem Alten zwei Karauschen.'

(KKN_1971_FiveCarpBrothers_flk_12, S: Upper Ket')

(132)

a. $[N \mathcal{E}-l]_{T} \quad[\mathrm{mekka}]_{R}$ me-d!

daughter..NOM-2SG 1SG.DAT give-IMP.2SG.O

'Gib mir deine Tochter!'

(ChDN_1983_HeroesDaughter_flk_34, Z: Vasjugan)

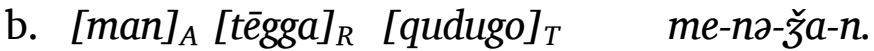

1SG 2SG.DAT pull.strap.NOM give-DRV-FUT-1SG.S

'Ich gebe dir einen Strick.'

(PVD_1961_FarmAssault_flk_45, S: Chaja)

Außer dem Agens können auch Rezipient und Thema lexikalisch kovert ausgedrückt werden. In allen Dialekten finden sich Beispiele mit koverten semantischen Rollen, wie in (133a) mit kovertem Thema oder in (133b) mit kovertem Agens und Rezpient. Das Thema erscheint einmal im Akkusativ, einmal unmarkiert. Es ist sogar möglich Agens, Rezpient und Thema lexikalisch kovert auszudrücken. In Beispiel (133c) sind Agens und Thema am Verb markiert (- $w$ - '1SG.O'), der Rezipient erschließt sich lediglich aus dem Kontext (Im Satz davor wird Itja aufgefordert, Pönegessa das Ruder zu geben).

a. $[M a t]_{A}[t \varepsilon k a]_{R}$ me-le-be na-tko.

1SG 2SG.DAT give-OPT-1SG.O this-PROL

'Ich gebe (ihn) dir dafür.'

(MNS_NN_BullSon_flk_14, Z: Narym)

b. Nändi-zä mē-za-t [daspehi-la-m bagatirskie $]_{T}$.

then = EMPH give-PST-3SG.O armor-PL-ACC hero's

'Dann gab (sie) (ihm) Heldenrüstungen.'

(AGS_1968_FairytaleSnake_flk_81, S: Middle Ket')

c. ... aza me-nža-w.

NEG give-FUT-1SG.O

'(Ich) gebe (es) (dir) nicht.'

(TFF_1967_IdjaAldigaNet_flk_23, S: Middle Ob)

Vereinzelt ist das Thema kein Nomen oder Personalpronomen, sondern ein Quantifizierer oder ein Demonstrativpronomen. In Beispiel (134) ist das Thema der Quantifizierer koti - 'viel'. Er trägt keine Kasusmarkierung, sondern erscheint mit einem Adverbialsuffix. Agens und Rezipient sind Personalpronomen. 
$\ldots[t a b]_{A}[t a b-l a-n i]_{R} \quad[k o t i-k]_{T} \quad m e-k-n \varepsilon-t$

3SG 3SG-PL-DAT/ALL much-ADV give-ITER-AOR-3SG.O

'[Dass] sie ihnen viel gibt.'

(ILP_1981_StonyOldWoman_flk_9, S: Middle Ob)

\section{tati}

Das zweite physikalische Transferverb, das in ditransitiven Konstruktionen erscheint, ist tati - 'bringen, geben'. Da es im Korpus häufig mit lexikalisch kovertem Rezipienten vorkommt, gibt es insbesondere im zentralselkupischen Subkorpus nur wenige Beispiele. In Beispiel (135a) ist der Rezipient das Personalpronomen der 1. Person mit dem Dativ markiert (meka), das Thema ist ein Akkusativobjekt. Die Beispiele (135b) und (135c) zeigen südselkupische Konstruktionen mit nominalem und pronominalem Rezipienten, das Thema ist jeweils mit dem Akkusativ kodiert.

a. Tad-le-l $\quad[m e k a]_{R}[\ddot{u} d-\dot{i}-p]_{T}, \quad$ mat üdc-šša-k. bring-OPT-2SG.O 1SG.DAT water-EP-ACC 1SG drink-DRV-1SG-S

'Bring mir Wasser, ich möchte trinken.' (KIA_2014_ItjaKet_trans_113, Z: Narym)

b. $[N a \text { sūru-p }]_{T} \quad t \bar{d} d \dot{t}-k k u-z \bar{a}-d i t \quad[t a b-n i]_{R}$. this wild.animal-ACC bring-ITER-PST-3PL 3SG-DAT

'Diese Tiere brachten (sie) ihr.'

(ILP_1981_StonyOldWoman_flk_5, S: Middle Ob)

c. $[\text { Pö́-j } \quad \text { paja-ni }]_{R} \quad t \bar{a} d \dot{t}-k k u-z \bar{a}-d i t \quad[q w \bar{\partial} l-p]_{T}$, stone-ADJZ old.woman-DAT bring-ITER-PST-3PL fish-ACC

$[\text { sūru-p }]_{T}$.

wild.animal-ACC

'Der Steinfrau brachten (sie) Fisch (und) Wildtiere.'

(ILP_1981_StonyOldWoman_flk_8, S: Middle Ob)

Analog zu Beispiel (134) enthält das Beispiel in (136) ein Demonstrativpronomen (tau - 'dieses') als Thema. Der Rezipient ist das Personalpronomen der 1. Person, der Agens ist lexikalisch overt ausgedrückt (mannani nan'au - 'meine Schwester').

$[\mathrm{tau}]_{T}[\mathrm{me} \bar{e} \mathrm{ja}]_{R}$ tas-si-t $\quad[\text { man-nani nan'a- } u]_{A}$.

this 1SG.DAT bring-PST-3SG.O 1SG-ADE sister-1SG

'Dies brachte mir meine Schwester.'

(PMP_1961_ForestWoman_flk_358, S: Middle Ob)

Innerhalb eines Satzes können mehrere unterschiedliche ditransitive Verben vorkommen. Beispiel (137) zeigt die physikalischen Transferverben tati - 'bringen' und mi - 'geben' sowie taw - 'kaufen', das in benefaktiven Konstruktionen verwendet wird. Die Rezipienten der beiden Transferben (mejga und kudneij) sind lexikalisch overt ausgedrückt, das Agens und das Thema aller ditransitiven Verben ist hingegen kovert. 


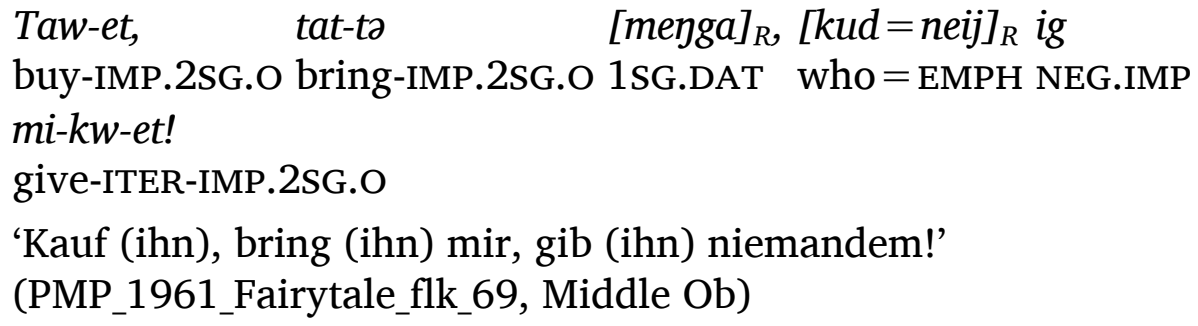

In ihrem Artikel beschreiben Wagner-Nagy/Szeverényi (2013) eindeutige Unterschiede in der Verteilung der Verben $m i$ - 'geben' und tati - 'bringen, geben' in den nordsamojedischen Sprachen und im Nordselkupischen, abhängig vom Rezipienten. So kann im Nganasanischen in Fällen, in denen Agens uns Rezipient identisch in Person und Numerus sind, ausschließlich das Verb tatud'a - 'geben, bringen' verwendet werden. Das Verb mis ${ }^{j} i$ - 'geben, zurückkehren' wird lediglich mit Rezipienten der 3. Person verwendet (ebd.: 28, 31). Im Nordselkupischen ist das Verb tati lediglich für die Kombination 3. Person Agens | 3. Person Rezipient belegt. In allen anderen Kombinationen ist ausschließlich mi belegt. Die Kombinationen Agens 1. Person | Rezipient 1. Person sowie Agens 3. Person| Rezipient 2. Person sind nicht belegbar.

Analysen im Selkup Language Corpus zeigen hier ein deutlich erweitertes Bild. In Tabelle 15 kann man errkennen, dass in den zentralen und südlichen Dialekten des Selkupischen beide Lexeme in praktisch allen Kombinationen (Person und Numerus) von Agens und Rezipient verwendet werden. In Fällen, in denen Agens und Rezipient hinsichtlich Person und Numerus übereinstimmen, ist im Korpus für die erste Person nur tati belegt, für die 2. Person nur mi. Es lässt sich nicht feststellen, ob diese Verteilung lediglich auf fehlendes Belegmaterial zurückzuführen ist oder ihr bestimmte Gesetzmäßigkeiten zugrunde liegen. Die Tatsache, dass in allen anderen Kombinationen von Agens und Rezipient beide Verben vorkommen, lässt jedoch ersteres als wahrscheinlich gelten.

Ebenfalls nicht mit Korpusdaten belegen lässt sich die von Wagner-Nagy/Szeverényi (ebd.) für das Nganasanische beobachtete Bedeutungsdifferenzierung für tatt. In Fällen mit identischem Agens und Rezipient erscheint tati dort nur mit der Bedeutung 'bringen' anstelle von 'geben' hat. Im Selkup Language Corpus finden sich in den originalen Übersetzungen beide Varianten, wobei einschränkend gilt, dass es für diese Fälle nur wenige Beispiele gibt und die russischen Übersetzungen nicht immer exakt sind.

Tab. 15: Verteilung von $m i$ - 'geben' und tati - 'bringen, geben'

\begin{tabular}{l|lll}
\hline Rezipient & 1. Person & 2. Person & 3. Person \\
Agens & & & \\
1. Person & tati & tati $\mid \mathrm{mi}$ & tati $\mid \mathrm{mi}$ \\
2. Person & tati $\mid \mathrm{mi}$ & $\mathrm{mi}$ & tati $\mid \mathrm{mi}$ \\
3. Person & tati $\mid \mathrm{mi}$ & tati & tati $\mid \mathrm{mi}$ \\
\hline
\end{tabular}




\section{qwande}

Das Verb qwande - 'bringen' ist das dritte der im Korpus belegten physikalischen Transferverben. Es kommt nur in einzelnen südselkupischen Beispielen in der Verwendung als ditransitives Verb vor. Im Zentralselkupischen ist es nicht belegt. Beispiel (138a) enthält einen nominalen Rezipienten, der mit dem Dativ markiert ist (pajani - 'alte.Frau.DAT') und ein nominales Thema im Akkusativ ( $q w \bar{z} l i p$ - 'Fisch.ACC'). In Beispiel (138b) sind Rezipient und Thema Pronomen (mazim, täbani), der Agens ist lexikalisch kovert und nur am Verb markiert.
a. $[P \bar{O}-j \quad \text { paja-ni }]_{R} \quad[q w \bar{l} l i-p]_{T}$ qwandi-kku-za-dit. stone-ADJZ old.woman-DAT fish-ACC carry.away-ITER-PST-3PL
'Der Steinfrau brachten sie Fisch.'
(ILP_1981_StonyOldWoman_flk_4, S: Middle Ob)
b. Qwända-k $\quad[\text { mazim }]_{T}[t a ̈ b-a-n i]_{R}$.
'Bring mich zu ihm.'
(ZNP_1964_Clock_trans_86, S: Middle Ket')
carry.away-IMP.2SG.O 1SG.ACC 2SG-EP-DAT

\section{Kognitive und mentale Transferverben}

Kognitive und mentale Transferverben bilden die zweite Gruppe von Verben, die im Selkupischen in ditransitiven Konstruktionen verwendet wird. In den zentral- und südselkupischen Subkorpora gibt es Beispiele für die Verben ati - 'zeigen', kät - 'erzählen, sagen' und ütz - 'schicken'. Im Unterschied zu Konstruktionen mit physikalischen Transferverben ist der Rezipient hier der Adressat der Handlung, die vom Agens ausgeführt wird. Die Markierung von Rezipient (bzw. Adressat) und Thema stimmt mit der von physikalischen Transferverben überein, der nominale oder pronominale Adressat ist also mit dem Dativ markiert, das Thema erscheint unmarkiert oder mit dem Akkusativ.

ati

Die Beispiele (139a) und (139b) enhalten das Verb att - 'zeigen' in den Varianten $\bar{a} d \varepsilon$ und $\bar{a} d u$. In beiden Beispielen sind Agens, Rezipient und Thema lexikalisch overt realisiert, die Themata (watep - 'Weg' und qwälikam - 'Fisch') sind mit dem Akkusativ markiert, das Agens erscheint einmal nominal (139a), in (139b) ist es ein Personalpronomen.

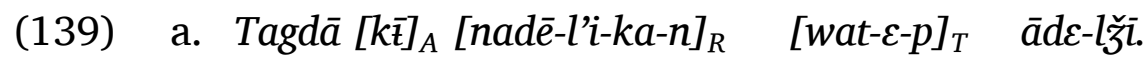

then river girl-DIM-DIM-DAT way-EP-ACC show-INT.PF.3SG

'Dann zeigt der Fluss dem Mädchen den Weg.'

(TTD_1964_WildDucks_flk_24, Z: Tym)

b. $[\mathrm{Man}]_{A}[\text { teja }]_{R} \quad \bar{a} d u-l \check{e} e-n \check{z} a-w \quad[q w \overline{a ̈ l} \mathrm{l}-k a-m]_{T}$ !

1SG 2sG.DAT show-INT.PF-FUT-1SG.o fish-DIM-ACC

'Ich werde dir den Fisch zeigen.'

(KMS_1968_Burbot_trans_112, S: Lower Ket') 
kät

Das Verb kät - 'erzählen' wird in den Beispielen (140a), (140b) und (140c) in den Varianten kadit, ket und kade verwendet. In (140a) und (140b) ist das Thema das Interrogativpronomen qaj - 'was' im Akkusativ, in (140c) ist es der aus dem Russischen entlehnte Quantifizierer wes, der hier als Adverbial verwendet wird.

a. A näde-k kur-a-nn, $\quad[q a j-m=n a j]_{T} \quad \bar{a} \quad k a d \dot{t}-d$ but girl-DIM run-AOR-3SG.S what-ACC = EMPH NEG tell-3sG.O $[t a b-\partial-n d]_{R}$. 3SG-EP-ILL

'Aber das Mädchen lief und sagte ihm nichts.' (SAA_1971_ThreeSisters_flk_59, Z: Narym)

b. $[K u \text {-la-tdi-ne }]_{R} \quad[q a i-m=n \bar{e}-s]_{T} \quad$ ket-ku-t. person-PL-OBL.3SG-DAT what-ACC $=$ EMPH-CLIT tell-ITER-3SG.O 'Seinen Leuten hat er nichts erzählt.' (PVD_1961_FarmAssault_flk_10, S: Chaja)

c. $[N \bar{\varepsilon}-t]_{A} \quad[w e s]_{T}$ kade-l-ba-t $[q \bar{u}-l a-n]_{R}$ daughter-3SG all tell-INCH-PST.REP-3SG.O person-PL-DAT [aze-nd-ni $]_{R}$. father-OBL.3SG-DAT

'Die Tochter erzählt alles den Leuten (und) ihrem Vater.' (TMR_1981_Robbers_flk_42, S: Middle Ob)

ütə

Die Beispiele (141a) - (141c) zeigen die Sprecher-Varianten $\ddot{u} d e$, üde sowie üta des Verbs ütz - 'schicken'. Das Thema kann nominal wie das aus dem Russischen entlehnte prikas - 'Befehl' in (141a) oder pronominal wie in (141b) erscheinen. Das Thema in (141c) ist ebenfalls ein Nomen. Es erscheint wie das Thema in (141a) unmarkiert.

a. [Mĩ wary mažo-l āmdeyo $]_{A},[q o r \gamma]_{A}$ üde-špa-d [tab-t 1PL big taiga-ADJZ tsar bear send-IPFV-3SG.O 3SG-GEN prikas $_{T} \quad[\text { wes' čumbenē-la-n }]_{R}$. command all wolf-PL-DAT

'Unser großer Wald-Zar, der Bär, schickt seinen Befehl allen Wölfen.' (TTD_1964_CalfHare_flk_30, Z: Tym)

b. $\left[\ddot{A} s e-n d a ̈-n e \quad[\ddot{s} e t]_{T} \quad \ddot{a d e}-n \check{a} a-k\right.$. father-OBL.3SG-ALL mother-OBL.3SG-DAT 2sG.ACC send-FUT-1SG.S 'Zu Vater und Mutter werde (ich) dich schicken.' (NN_1855_Hero1_song_4, Z/S: Tym/Middle Ob)

c. $[\text { Iwan }]_{A}[q o j \text { qu-la-nne }]_{R} \quad[q u ̈ t t i]_{T}$ üta-ndi-t, a [b'ednaj Iwan rich person-PL-DAT illness send-INFER-3SG.o but poor qu-la-nne $]_{R} \quad[\text { so ila-say }]_{T}$. person-PL-DAT good live-INSTRN 
'Iwan schickte den reichen Menschen Krankheit, aber den armen Menschen ein gutes Leben.'

(KKN_1971_IvanAndGod_flk_40, S: Upper Ket')

\section{Benefaktive Konstruktionen}

Den dritten Typ bilden benefaktive Konstruktionen, in denen der Rezipient Nutznießer ${ }^{3}$ der Handlung ist. Wie in Konstruktionen mit Transferverben ist der Rezipient mit dem Dativ markiert, das Thema erscheint mit dem Akkusativ markiert, Nominativobjekte sind nicht belegt. Im Belegkorpus gibt es Beispiele für die Verben süt - 'nähen' wie in (142) und taw - 'kaufen' wie in (143a) und (143b). Im Zentralselkupischen Subkorpus erscheint 'nähen' fast ausschließlich in monotransitiven Konstruktionen, im südselkupischen Subkorpus finden sich Beispiele für mono- und ditransitive Konstruktionen.

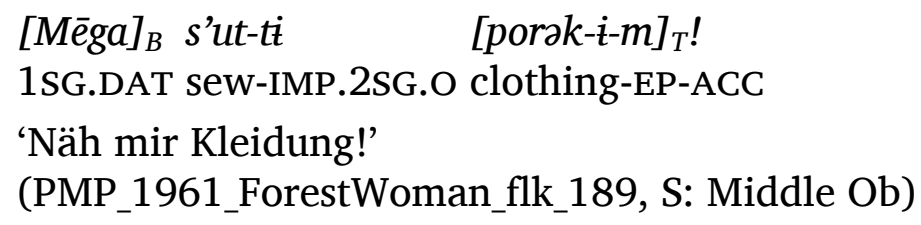

$[M \bar{e} g a]_{B}$ s'ut-tit $\quad[p o r \partial k-\dot{t}-m]_{T}$ !

1SG.DAT sew-IMP.2SG.O clothing-EP-ACC

'Näh mir Kleidung!'

(PMP_1961_ForestWoman_flk_189, S: Middle Ob)
a. $[M \bar{a} \check{s} a-n]_{B} \quad[f a \quad k a w p i-p]_{T}$ taw-i-mba-det.
Masha-DAT good cloth-ACC buy-EP-PST.REP-3PL.
'Sie kauften Mascha einen schönen Schal.'
(TTD_1964_WildDucks_flk_52, Z: Tym)

b. Tan moziš täb-a-m mir-a-yu i taw-wi-gu

2SG can.2SG 3SG-EP-ACC sell-EP-INF and buy-AOR-INF

[onnäy-dä-ni $]_{B} \quad$ [qai-m-e-mma] $]_{T}$.

himself-OBL.3SG-DAT what-ACC-EP-INDEF

'Du kannst sie verkaufen und dir selbst irgendetwas kaufen.'

(ZNP_1964_Clock_trans_45, S: Middle Ob)

Im vorangegangen Abschnitt wurden intransitive und transitive Deklarativsätze beschrieben. Das Subjekt in (in)transitiven Sätzen erscheint im Nominativ. Aufgrund des Pro-Drop-Charakters kann ein pronominales Subjekt lexikalisch kovert sein. In den zentral- und südselkupischen Dialekten zeigt sich die differentielle Objektmarkierung in mono- und ditransitiven Konstruktionen (bei letzteren nur innerhalb des indirektiven Typs, siehe hierzu auch die Einleitung 4.1.2.2 auf Seite 102), indem das direktes Objekt bzw. Thema entweder mit dem Akkusativ markiert oder unmarkiert (d.h. dem Nominativ markiert) erscheint. Klare und eindeutige Regeln für die KasusVerwendung direkter Objekte sind bisher nicht formuliert. Gerade in südselkupischen Texten werden in ähnlichen Umgebungen sowohl Akkusativ- als auch Nominativobjekte verwendet.

Nur in den nordselkupischen Dialekten findet sich ein zusätzlicher Ausrichtungstyp.

\footnotetext{
${ }^{3}$ Der Nutznießer wird mit „B“ abgekürzt.
} 
Das Thema des ditransitiven Satzes wird anders - nämlich mit dem Instrumental markiert als das Patiens (des monotransitiven Satzes) und anders als der Rezipient (secondary object alignment bei Malchukov/Haspelmath/Comrie (2010)). Der Rezipient wird wie das Patiens markiert, mit dem Akkusativ. Das Muster erscheint sowohl mit nominalen als auch mit pronominalen Rezipienten, ein Beispiel zeigt (144).

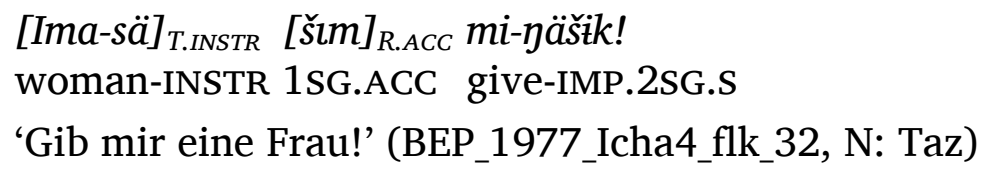

Im Selkupischen können ditransitive Konstruktionen mit physikalischen ( $m i$ - 'geben', tati - 'bringen', qwand - 'tragen' und ü̈t - 'schicken') sowie kognitiven und mentalen Transferverben ( $a t \dot{t}$ - 'zeigen', kät - 'erzählen' und ütə - 'schicken') gebildet werden. Darüber hinaus beinhalten auch benefaktive Konstruktionen diese Struktur. Die Argumente ditransitiver Verben können lexikalisch overt realisiert werden, wie in den Beispielsätzen (129a), (130a) und (130b), müssen es jedoch nicht, wie in (131a) und (133c). In letzterem ist nur das Verb lexikalisch realisiert, Agens, Patiens und Thema sind kovert. Das Thema ist in der Regel ein Nomen (Beispiele (135a) und (139a)), kann jedoch auch als Personalpronomen (Beispiel (141b)) bzw. Demonstrativpronomen (Beispiel (136)) oder Adverb (Beispiel (134)) realisiert werden. Wie in monotransitiven Sätzen kann das Objekt bzw. Thema auch unmarkiert erscheinen (Beispiel (133b)).

Affirmative intransitive sowie (mono- und di-) transitive Sätze können mit Hilfe der Negationspartikel ašša bzw. ihren Varianten negiert werden. Die Negationspartikel wird dabei der finiten Verbform vorangestellt. Das Subjekt (intransitiver Satz) bzw. das Subjekt und das Objekt (monotransitiver Satz) können lexikalisch overt ausgedrückt sein oder kovert erscheinen (Beispiele (196a) - (196c) und (??) - (??)), Gleiches gilt für die semantischen Rollen in ditransitiven Konstruktionen (Beispiele (198a) $-(198 c))$.

\subsubsection{Kopulasätze}

Kopulasätze enthalten typischerweise die Kopula $\bar{e}$ - 'sein' als Prädikat sowie zwei Komplemente - Kopula-Subjekt und Kopula-Objekt. Belegt sind auch Beispiele, in denen die Verben $\varepsilon s \dot{i}$ - 'werden' und orim - 'wachsen' als Kopula fungieren. Anders als in intransitiven oder transitiven Konstruktionen können mittels Kopulasätzen unterschiedliche Relationen ausgedrückt werden. Die Funktion hängt zum Großteil von der Art des Kopula-Objektes ab. Die in Tabelle 16 dargestellten Funktionen von Kopulasätzen im Selkupischen basieren auf Dixon (2010b: 159). In ihnen fallen die von Payne (1995: 111ff.) unterschiedenen predicate nominals, predicate adjectives und predicate locatives zusammen. Im Gegensatz zu Payne werden Konstruktionen, die keine overt ausgedrückte Kopula enthalten, getrennt in Kapitel 4.1.4 auf Seite 116 beschrieben. 
Wird als Komplement der Kopula eine Nominalphrase im Nominativ verwendet, wird das grammatische Subjekt klassifiziert oder identifiziert (nominales Prädikat), wie in den Beispielen (145a) - (145c). In (145b) und (145c) wird mit den Varianten $\bar{\varepsilon} \breve{z} i$ und ässu des Verbs 'werden', das hier als Kopula verwendet wird, eine translativische Bedeutung ausgedrückt.
a. Ad'a-de $[e-p p i-m b a]_{C O P} \quad[m e n e-r-t i l \quad q u p]_{N P . N O M}$. father-3SG be-HAB-PST.REP.3SG hunt-FRQ-ADJZ person
'Der Vater war ein Jäger.'
(ChDN_1983_ItjaStayedAlone_flk_2, Z: Vasjugan)

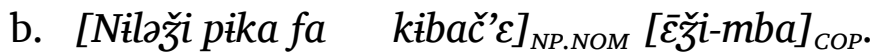 such bull good boy become-PST.REP.3SG
'Der Stier ist solch ein schöner Jüngling geworden.' (MNS_NN_BullSon_flk_86, Z: Narym)
c. A täp [nilž̉isō kündä] $]_{N P . N O M}[\ddot{a} s s u-z a-n]_{C O P}$. and 3SG such good horse become-PST-3SG.S
'Und es wurde solch ein gutes Pferd.'
(AGS_1968_FairytaleSnake_flk_80, S: Middle Ket')

Tab. 16: Semantische Relationen in Kopulasätzen

\begin{tabular}{ll}
\hline Kopula-Objekt & Relation \\
\hline $\mathrm{NP}_{\mathrm{NOM}}$ & Identität, Zustandsänderung \\
AdjP & Attribution \\
$\mathrm{PtcpP}$ & Attribution \\
$\mathrm{AdvP}$ & Attribution \\
$\mathrm{NP}_{\text {INSTR }}$ & Attribution \\
$\mathrm{NP}_{\mathrm{CAR}}$ & Attribution \\
$\mathrm{NP}_{\mathrm{GEN}}$ & Possessivität \\
$\mathrm{NP}_{\text {LOC }}$ & Possessivität, Lokation, Existenz \\
$\mathrm{NP}_{\mathrm{TRL}}$ & Zustandsänderung \\
$\mathrm{AdvP}_{\text {LOC }}$ & Lokation \\
\hline
\end{tabular}

Das Komplement kann aus einer oder mehreren Adjektivphrasen bestehen, wie in (146a) und (146b), die dem grammatischen Subjekt Eigenschaften zuweisen. Die prototypische Kopula $\bar{e}-$ 'sein' erscheint satzfinal in den Varianten $e$ bzw. $\varepsilon$, sie ist in beiden Beispielsätzen lexikalisch overt ausgedrückt. In Beispiel (146c) fungiert das Verb ē̌̌i - 'werden' als Kopula und formt eine translativische Bedeutung.
a. Nate-git hel'd' i-d amda-dit $[\mathrm{hag}]_{A D J P}\left[n^{\prime} \text { 'agdi }\right]_{A D J P},[n \text { 'arg }]_{A D J P}$ there-LOC.ADV seven son-3SG sit-3PL black bare red $[\varepsilon-j a-d i t]_{C O P}$. be-AOR-3PL


'Dort sitzen die sieben Söhne, sie sind schwarz, nackt und rot.' (ChDN_1983_Pora_flk_44, Z: Vasjugan)

b. $N a[s u w a]_{A D J P}[e-l e-s]_{C O P}$. this good be-OPT.3SG-CLIT

'Dies wird gut sein.'

(NN_1855_Hero2_song_35, Z/S: Tym/Middle Ob)

c. $[N i l ' c ̌ i-k \quad q w \bar{e} c ̌ d i]_{A D J P}[\bar{e} \check{z} \mathfrak{i}-m b a]_{C O P}$. such-ADV beautiful become-PST.REP.3sG

'So schön war sie geworden.' (TTD_1964_Frog_flk_60, Z: Tym)

Mit einem adjektivischen Prädikat können im Selkupischen (wie in anderen uralischen Sprachen auch) komparative Konstruktionen gebildet werden. Komparative Partikeln gibt es dagegen nicht. Die Wortfolge ist typischerweise Komparativ-NP $\mathrm{Nom}_{\text {nom }}$ + Standard-NP $\mathrm{abl}+$ Adjektiv, wie in (147b). In Beispiel (147a) steht die Standard-NP satzfinal. In beiden Beispielen ist auch denkbar, dass das Suffix -nan keine Variante des Ablativs nanni ist, sondern ein Adessiv (vgl. hierzu auch die Ausführungen auf Seite 24).

(147) a. Peqa-n wad'z' $[s \bar{o}]_{A D J P}[e-j a]_{C O P}$ mi wad'z'i-nan.

elk-GEN meat good be-AOR.3SG 1PL meat-MEAT-EL2

'Elchfleisch ist besser als unser Fleisch.'

(KFN_1967_BigBear_flk_10, Z: Tym)

b. Köy tim-nan [ütčuga $]_{A D J P}[\bar{e}-k]_{C O P}$.

river.Kenga river.Tym-EL2 small be-3SG.S

'Die Kjonga ist kleiner als der Tym.'

(KFN_1967_Mammoth_flk_7, Z: Tym)

Partizipien fungieren im Selkupischen wie Adjektive Eine Partizipphrase kann als Komplement eines Kopulasatzes auftreten, in Beispiel (148) ist sie overt ausgedrückt und steht vor dem Komplement.

(148) Tep $[\bar{e}-s s a-n]_{C O P}$ [qappərə-mbədi $]_{P T C P P}$.

3SG be-PST-3SG.S become.fat-PTCP.PST

'Es war fett.'

(KMS_1966_MouseGray_flk_12, S: Middle Ket')

Das Komplement kanp aus einer (abgeleiteten) Adverbphrase bestehen, wie in den Beispielen (149a) und (149b). Ebenso wie in (146a) - (146c) werden dem grammatischen Subjekt Eigenschaften zugeschrieben. Die Kopula ist auch hier ausgedrückt und erscheint satzfinal.

a. Mat qai [hela-n $]_{A D V P}[e-j a-k]_{C O P}, \quad \ldots$

1SG what whole-ADV be-AOR-3SG.S

'(Ob) ich heil bin, ...'

(ChDN_1983_ItjaStayedAlone_flk_26, Z: Vasjugan) 


\section{b. Inne-za-t tü-ya-tit, tutto-la [taniqin $]_{A D V P}[e-\gamma a-t]_{C O P}$. brother-CRC-PL come-AOR-3PL crucian-PL little be-AOR-3PL \\ 'Die Brüder kamen, die Karauschen sind weniger (geworden).' (KKN_1971_FiveCarpBrothers_flk_26, S: Upper Ket')}

Ebenfalls attributive Funktion haben Komplemente in Form einer InstrumentalNP oder einer Karitiv-NP, wie in den Beispielsätzen (150a) und (150b) bzw. (151). In (151) wird die Kopula $\bar{e}-$ 'sein' verwendet, in (150b) die Kopula-Variante $e$, in (150a) fungiert das Verb azi - 'werden' als Kopula. Die Komparativ-Bildung mit Ablativ (bzw. Elativ) ist typisch für viele uralische Sprachen, im Selkupischen sind jedoch nur einzelne Beispiele belegt. In Beispiel (150b) wird die für viele uralische Sprachen typische Komparativ-Bildung mit Ablativ (bzw. Elativ) verwendet, um mit Hilfe der Instrumental-NP oruz - 'mit Kraft' und der Ablativ-NP surulanannz - 'von den Tieren' den Superlativ auszudrücken. Aufgrund der sehr wenigen Komparationsbeispiele überhaupt lässt sich die Struktur nicht detaillierter betrachten.

a. ..., što $n \bar{\varepsilon}-t \quad[\text { tar-ZzE }]_{N P . I N S T R}[a z \dot{z}-m b a]_{C O P}$.

what daughter-3SG hair-INSTR become-PST.REP.3SG

'..., dass ihre Tochter behaart wurde.'

(TMR_1981_Devil_flk_40, Middle Ob)

b. Sūru [or-u-z $]_{\text {NP.INSTR }}[e-\eta]_{\text {COP }}$ suru-la-nanna.

wild.animal force-EP-INSTR be-3SG.S wild.animal-PL-EL2

'Der Bär (wrtl.: wildes Tier) ist das stärkste von allen Tieren.'

(BNN_1971_DogSearchedMistress_flk_26, S: Middle Ket')

(151) Tab [haj-galim $]_{N P . C A R}[e-j a]_{C O P}$.

3SG eye-CAR be-AOR.3SG

'Er ist blind (wrtl.: ohne Auge).'

(ChDN_1983_Nikita_flk_28, Z: Vasjugan)

In Kombination mit dem Translativ sind Beispiele belegt, in denen neben dem Verb $\varepsilon z \dot{i}$ - 'werden' weitere Verben desemantisiert sind und als Kopula fungieren. Aufgrund von fehlenden weiteren Beispielen lässt sich nicht sagen, wie weit der Grammatikalisierungsprozess (lexikalisches Verb $->$ Hilfsverb) bereits fortgeschritten ist. Mit diesen Konstruktionen wird die Veränderung eines Zustandes ausgedrückt. In (04:cop.trl:01) fungiert das Verb ed'e - 'werden' (eine Variante von csi) als Kopula, in (04:cop.trl:02) ist es orim - 'wachsen', in (152c) wird kand'e - 'gefrieren' verwendet, um eine Zustandsänderung auszudrücken. Die Komplement-NP ist mit dem Translativsuffix -yle markiert, einer Variante von -wle, das nur in südselkupischen Dialekten belegt ist.

a. El'mat-te [mad'e-t qu-tko] $]_{N P . T R L}[e d ' e-l l a-d e]_{C O P}, \quad \ldots$ child-PL taiga-GEN person-TRL become-OPT-3PL

'Die Kinder werden zu Waldgeistern, ...'

(ChDN_1983_TaleBrownies_flk_24, Z: Vasjugan) 
b. Tab [wargi üčed'e-l'i-ka-tko] $]_{N P . T R L}[\text { orom-ba] }]_{C O P}$, 3SG big child-DIM-DIM-TRL grow.up-PST.REP.3SG

'Er ist zu einem älteren Burschen geworden (wrtl.: gewachsen), ...' (ChDN_1983_ItjaStayedAlone_flk_4, Z: Vasjugan)

c. A nan'a-kə-t [pü-j lozi- $-\gamma l$ l'e $]_{N P . T R L}[k a n d ' e-d ' i-m p a-n]_{C O P}$. but sister-DIM-3SG stone-ADJZ devil-TRL freeze-IPFV-PST.REP-3SG.S

'Aber ihre kleine Schwester hat sich in einen Steinteufel verwandelt (wrtl.: gefroren).'

(PMP_1961_ForestWoman_flk_124, S: Middle Ob)

Mit Hilfe einer Genitiv-NP als Komplement eines Kopulasatzes wird Possessivität (Genitive Schema bei Heine (2009: 58), Genitive Possessive bei Stassen (2001: 956)) ausgedrückt. Beispiele dieser Art kommen im Belegkorpus eher selten vor, typischer sind Konstruktionen mit einer Lokativ-NP (siehe Beispiele (154a) und (154b) unten). Die Stellung der Kopula ist nicht fest, in (153a) erscheint sie satzfinal, in (153a) zwischen Komplement und Subjekt. Der zweite Teilsatz in (153a) weist eine elliptische Konstruktion auf, das Genitiv-Komplement ist hier ausgelassen.

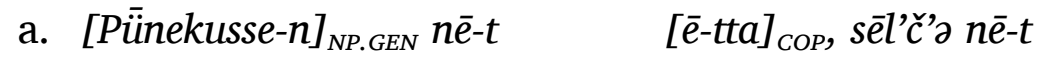

Pönegesse-GEN daughter-PL be-3PL seven daughter-3SG

$[\bar{e}-a-t]]_{C O P}$.

be-AOR-3SG.O

'Pönegesse hat Töchter, (er) hat sieben Töchter.'

(NN_1879_Itja_flk_87, S: Chaja)

b. $[\text { Tab-i-n }]_{N P . G E N}[\varepsilon-j a]_{\text {cop }}$ nagur $n \mathcal{E}-d$.

3SG-EP-GEN be-AOR.3SG three daughter-3SG

'Er hatte drei Töchter.'

(MNS_NN_BullSon_flk_20, Z: Narym)

Im Selkupischen können die Relationen Lokation, Possessivität und Existenz mit Lokativ-NPs als Komplement in Kopulasätzen ausgedrückt werden. Existential- und Lokativsätze sind von Budzisch (2017a) für die nord-, zentral- und südselkupischen Dialekte erstmals systematisch untersucht und beschrieben worden. Die typische Funktion einer Lokativ-NP als Komplement ist der Ausdruck der Lokation des Subjekts wie in (154a) und (154b). In Abgrenzung zu Existentialsätzen ist die Lokalangabe in Lokativsätzen obligatorisch, zudem trägt das Subjekt/Thema stets dann eine definite Markierung, wenn sie von der für Lokativsätze typischen Wortfolge th + LOC + COP abweicht. Das kann ein Possessivsuffix sein wie in (154a) oder das Demonstrativpronomen na wie in (154b). Neben einer Lokativ-NP kann auch ein lokales Adverb als Komplement verwendet werden wie nač'āt - 'dort' in (154a) oder taw - 'hier' in Beispiel (155).
a. $[N a c ̌ a ̄ a t]_{N P . L O C} t ' e m n ' a-m \quad[e-t t a]_{C O P}$.
there
brother-1SG be-IPFV.3sG 
'Mein Bruder ist dort.'

(MNS_1984_BrotherSister_flk_16, Z: Narym)

b. Na tu [n'är-t kojo-kot $]_{N P . L O C}[\bar{e}-k]_{C O P}$.

this lake swamp-GEN near-LOC be-3SG.S

'Dieser See ist am Sumpf gelegen.'

(KFN_1967_HumanSizedPike_flk_10, Z: Tym)

(155) Mat $[\text { taw }]_{A D V . L O C}[e-a-k]_{C O P}$.

1SG here be-AOR-1SG.S

'Ich bin hier.'

(MNN_1977_ItjaGrandmother_flk_21, Z: Tym)

Die zweite Relation, die mit einer Lokativ-NP ausgedrückt werden kann ist die der Existenz. Im Gegensatz zu nordsamojedischen Sprachen, in denen Existential- und Lokativsätze (neben der Wortstellung) auch mit Hilfe zweier unterschiedlicher verbaler Elemente (Existenzialverb im ersten, sein-Verb im zweiten Fall) unterschieden werden, verfügt das Selkupische über kein affirmatives Existentialverb. Existentialsätze werden daher ebenfalls mit der Kopula $\bar{e}$ ausgedrückt. Negierte Existenzialsätze, in denen die Negation mit Hilfe eines negativen Existenzialverbs ausgedrückt wird, sind im Anschluss an die affirmativen Sätze beschrieben. Die Unterscheidung im affirmativen Satz erfolgt ausschließlich über die Wortstellung, die in Existenzialsätzen die Abfolge: Lokation (LOC) - Subjekt/Thema (TH) - Kopula (COP) aufweist (Wagner-Nagy 2016: 208). Im Gegensatz zu Lokativsätzen ist die Lokalangabe in Existentialsätzen fakultativ. Die Beispielsätze (156a) und (156b) enthalten auch Lokalangaben, die Sätze (156c) und (156d) dagegen nicht. Die Kopula erscheint zumeist satzfinal, sie kann jedoch auch in anderen Positionen erscheinen, wie in (156c) vor dem Subjekt/Thema.

a. [Nat'e-yit $]_{N P . L O C}$ tudo-t ukkir haj-he [e-ja-dit $]_{C O P}$. there-LOC.ADV crucian-PL one eye-INSTR be-AOR-3PL

'Dort gibt es Karauschen mit einem Auge.'

(ChDN_1983_Nikita_flk_5, Z: Vasjugan)

b. [Čă̌akí-yit $]_{N P . L O C}$ waryi pirgi mad kit-t par-git $[e-j a]_{C O P}$. Chizhapka-LOC big high ravine river-GEN top-LOC be-AOR.3sG.

'Am Chizhapka gibt es einen hohen steilen Abhang.' (ChDN_1983_ItjasTown_flk_1, Z: Vasjugan)

c. Č' $\bar{e}$ tal'̌̌̆el $[e-\gamma a]_{C O P}$ nädek, ... so yesterday be-AOR.3SG girl

'So, gestern gab es ein Mädchen, ...'

(SAA_1971_ThreeSisters_flk_51, Z: Narym)

d. SwEšk koti- $\eta \quad[\bar{\varepsilon}-j a]_{C O P}$.

cedarwood.cone much-ADV be-AOR.3SG

'Es gibt viele Zedernzapfen.'

(SMS_1980_ItjaForest_flk_29, S: Middle Ob) 
Da das Selkupische kein „habeo“-Verb kennt, wird mit Lokativ-Konstruktionen auch Possessivität ausgedrückt (Location Schema bei Heine (2009: 50), Locational Possessive bei Stassen (2001: 955)). Possessivsätze weisen die gleiche zugrundeliegende Struktur wie Existenzialsätze auf (vgl. Lyons 1969: 390, Freeze 1992: 553). Der (formale) Unterschied zwischen beiden Relationen liegt neben der obligatorischen Lokalangabe (Possessor) in Possessivsätzen in der Animatheit des Lokatiy-Komplements: in Possessivsätzen ist es animat (tudonnan - 'Karausche.LOC' in (157a) oder tebistajenen - 'sie.LOC' in (157b)). In Existenzialsätzen ist das lokale Element, sofern overt ausgedrückt, inanimat wie Čažakizyit - 'Chizhapka.LOC' in Beispiel (156a) oben.

$$
\begin{aligned}
& \text { a. ... }\text { [tudo-nnan }]_{N P . L O C} \text { šidi haj }[e-j a]_{C O P} \\
& \text { crucian-ADE two eye be-AOR.3SG } \\
& \text { '... die Karausche hatte zwei Augen.' } \\
& \text { (ChDN_1983_Nikita_flk_7, Z: Vasjugan) } \\
& \text { b. } \\
&\text { [Teb-i-sta-ye-nen] } \left.]_{N P . L O C} \text { oqir kiban'aza [je-s] }\right]_{C O P .} \\
& \text { 3SG-EP-DU-DU-ADE one boy be-PST.3SG } \\
& \text { 'Sie hatten einen Jungen.' } \\
& \text { (PVD_1966_BoyDevil_flk_2, S: Chaja) }
\end{aligned}
$$

\section{Zusammenfassung}

Zusammenfassend kann festgestellt werden, dass mit Hilfe von Kopulasätzen im Selkupischen eine Vielzahl von Relationen wiedergegeben werden können. Die Art der Relation ist zum Teil vom Charakter des Kopula-Komplements abhängig, So wird Identität nur mit (pro)nominalen Phrasen im Nominativ ((145a), (159a)) ausgedrückt, Zustandsänderungen können jedoch mit unterschiedlichen Komplementen (Nominalphrasen im Nominativ (145b) oder Translativ (152a)) ausgedrückt werden. Auch Attribution, Possessivität und Lokation können mit mehr als einem Komplement ausgedrückt werden. Erstere mit Adjektivphrasen wie in (146a), Partizipphrasen wie in (148), Adverbphrasen wie in (149a) sowie Nominalphrasen im Instrumental wie in (150a) oder Karitiv wie (151). Zweiteres kann mit Nominalphrasen im Genitiv (Beispiel (153a)) und Lokativ (Beispiel (154a)) wiedergegeben werden, letzteres ebenfalls mit Nominalphrasen im Lokativ wie in (154b) sowie Adverben mit Lokalkasus wie in (155).

\subsubsection{Verblose Sätze}

Ebenso wie Kopulasätze drücken verblose Sätze unterschiedliche Relationen (vgl. Tabelle 16 in Abschnitt 4.1.3 auf Seite 110) aus. Anders als diese enthalten sie keine Kopula (Dixon 2010b: 160), verfügen jedoch über zwei Argumente (Verbless Clause Subject und Verbless Clause Object bei Dixon (2010b: 160)). Tabelle 17 zeigt die Kopula-Objekte sowie die Relationen, die mit ihnen in verblosen Sätzen ausgedrückt werden. 
Tab. 17: Semantische Relationen in Kopulasätzen

\begin{tabular}{ll}
\hline Kopula-Objekt & Relation \\
\hline $\mathrm{NP}_{\text {NOM }}$ & Identität, Zustandsänderung \\
PronP & Identität, Zustandsänderung \\
$\mathrm{AdjP}$ & Attribution \\
$\mathrm{PtcpP}$ & Attribution \\
$\mathrm{AdvP}$ & Attribution \\
$\mathrm{NP}_{\text {INSTR }}$ & Attribution \\
$\mathrm{NP}_{\text {LOC }}$ & Possessivität, Lokation, Existenz \\
AdvP $_{\text {LOC }}$ & Lokation \\
\hline
\end{tabular}

Ebenso wie in Kopulasätzen wird mit Hilfe von Nominativkomplementen in verblosen Sätzen eine Identität ausgedrückt. Im Selkupischen können dabei die verbalen Personalendungen direkt an das Objekt-Komplement angeschlossen werden, wie im zentralselkupischen Beispiel (158a), in dem das Suffix der zweiten Person Plural -lit direkt an das Objekt-Komplement cumilqub - 'Chumylkupe' angehängt ist. In Beispiel (158b) können Subjekt und Objekt morphologisch nicht unterschieden werden, lediglich der Kontext sowie die satzinitiale Stellung des Subjektes (man - 'ich') geben darüber Auskunft.
a. Ti [čumill-qub-lit $]_{\text {NP.NOM. }}$
2DU Selkup-person-2PL
'Ihr (seid) Chumylkupen.'
(ChDN_1983_MistressOfFire_flk_76, Z: Vasjugan)
b. Man $[\mathrm{ta}-\gamma \circ \mathrm{j}]_{\text {NP.NOM. }}$.
1SG tatar-prince
'Ich (bin) der Tatarenfürst.'
(NN_1913_Itja_flk_12, S: Middle Ob)

Das Komplement kann eine Pronominalphrase sein, wie das emphatische Pronomen in Beispiel (159a). Das Beispiel enthält im ersten Teilsatz außerdem eine weitere Kopulakonstruktion mit einer Nominativ-NP als Komplement. In Beispiel (159b) ist das Komplement das Interrogativpronomen kuti - 'wer', in der Variante kudo. Wie čumil$q u b$ in (158a) trägt es eine Verbmarkierung (der zweiten Person Singular). Beispiele dieser Art sind nur mit koverter Kopula belegt.

a. Te [qot'i-ya-t] $]_{N P . N O M}$, man [ojey $]_{E M P H}$.

2PL much son-DIM-3SG 1SG myself

'Ihr (seid) viele Söhne, ich (bin) allein.'

(KKN_1971_FiveCarpBrothers_flk_102, S: Upper Ket')

b. Tat [kudo-nt?] $]_{\text {INTERROG }}$

2SG who-2sG.S 
'Wer bist du?'

(ChDN_1983_TaleBrownies_flk_18, Z: Vasjugan)

Das Komplement kann aus einer (Beispiel 160a) oder mehreren (Beispiele 160b) Adjektivphrasen bestehen, die dem grammatischen Subjekt Eigenschaften zuweisen. Die attributiven Prädikate qoj - 'reich' sowie wary - 'groß' und fa - 'schön' stehen satzfinal.

a. Tü $[q o j]_{A D J P} \ldots$

fire rich

'Das Feuer (ist) reich ...'

(KKN_1971_FiveCarpBrothers_flk_89, S: Upper Ket')

b. Škola mi-nan $[\text { wary }]_{A D J P},[f a]_{A D J P}$.

school 1PL-ADE big good

'Unsere Schule (ist) groß und schön.'

(TTD_1964_PlaceWhereILive_nar_4, Z: Tym)

Da Partizipien im Selkupischen wie Adjektive fungieren, weisen auch sie dem grammatischen Subjekt Eigenschaften zu. Im zentralselkupischen Beispiel (161) ist das Subjekt das emphatische Pronomen onž - 'selbst', das attributive Prädikat qwežidil 'schön' ist mit einem Adverb modifiziert. Südselkupische Beispiele sind nicht belegt.

(161) Onž našan [qweži-dill $]_{P T C P P}$.

himself so.much be.beautiful-PTCP.PRS

'Sie selbst (ist) so schön.'

(MNS_1984_BrotherSister_flk_58, Narym)

In Beispiel (162) aus dem südselkupischen Ket'-Dialekt ist das Komplement ein abgeleitetes Adverb (pirgey - 'groß'), das Subjekt wird durch das Personalpronomen täp - 'er' ausgedrückt. Zentralselkupische Beispiele sind nicht belegt.

(162) Onda täp [pirg-e- $\eta]_{\text {PRONP. }}$.

himself 3SG tall-be-3SG.S

'Er selbst ist groß.'

(KMS_1967_Elk_nar_2, S: Middle Ket')

Komplemente in Form einer Instrumental-NP, die eine attributive Funktion haben, sind in verblosen Sätzen nur in einem Beispiel belegt. Das Instrumental-Objekt in (163) ist orfessi - 'mit Kraft', das Subjekt madur - 'Held' erscheint satzintial. Ähnliche Beispiele mit overt ausgedrückter Kopula finden sich in (150a) und (150b).

(163) Madur [orf-e-ssi] $]_{\text {NP.INSTR }}$.

hero force-EP-INSTR

'Der Held ist stark.'

(NN_1855_Hero1_song_87, Z/S: Tym/Middle Ob) 
Die im vorigen Abschnitt 4.1.3 beschriebene Möglichkeit, lokative, possessive und existenzielle Relationen mit Lokativ-Komplementen auszudrücken, gilt auch für verblose Sätze. Lokalsätze weisen dabei die Wortfolge TH + LOC auf, Existenzial- und Possessivsätze die Wortfolge LOC + TH. Die Beispiele (164a) - (164c) zeigen Lokativsätze, die Lokalangabe kann ein Nomen sein wie mogoyint - 'im Rücken' in (164a), ein Personalpronomen wie tepannan - 'bei ihm' in (164b) oder eine relationale Konstruktion wie t'ūn pūčoyan - 'in der Erde' sein.
a. Pönaga [mogo-yint $t_{N P . L O C}$.
Pönege back-LOC.3SG
'Pönege ist hinten.'
(KFN_1967_BigBear_flk_11, Z: Tym)
b. Oka qum [tep-a-n-nan $]_{N P . L O C}$.
one person 3SG-EP-GEN-ADE
'Ein Mensch ist bei ihm.'
(KKN_1971_IvanAndGod_flk_3, S: Upper Ket')
c. Teb-i-n pida-t $\left[t^{\prime} \bar{u}-n \text { pūčo-yan }\right]_{N P . L O C}$.
3SG-EP-GEN nest-3SG earth-GEN inside-LOC
'Sein Nest ist in der Erde.'
(KMS_1966_MouseGray_flk_5, S: Middle Ket')

Die Existenzialsätze in den Beispielen (165a) und (165b) weisen die typische Wortfolge LOKATION - THEMA auf. Dabei kann die Lokalangabe mit Hilfe eines lokalen Adverbs (Beispiel (165a)) oder einer Lokativ-NP (Beispiel (165b)) ausgedrückt werden.
a. [Nat'e-yit $]_{N P . L O C}$ wiril haj-gidit-l ära.
there-LOC.ADV harmful eye-CAR-ADJZ old.man
'Dort ist ein böser, einäugiger alter Mann.'
(ChDN_1983_Nikita_flk_12, Z: Vasjugan)
b. [Okkir tšotšo-qon] $]_{N P . L O C}$ ïbqaj qwaj.
one place-LOC big big.river
'An einem Ort ist ein großer Fluss.'
(PMP_1961_Fairytale_flk_236, S: Middle Ob)

Possessivsätze weisen die gleiche Struktur auf wie Existenzialsätze. Anders als in diesen ist das Lokativ-Komplement in Possessivsätzen jedoch stets animat. Wie in Kopulasätzen wird in den Beispielen (166a) und (166b) der Possessor mit einem pronominalen Lokativ-Komplement (Location Schema bei Heine (2009: 50), Locational Possessive bei Stassen (2001: 955)) ausgedrückt, die morphologische Markierung erfolgt mit dem Adessiv (manan - 'bei mir' bzw. Tabnan - 'bei ihm'). In beiden Beispielen ist das Possessum mit einem Possessivsuffix markiert, das mit dem Subjekt hinsicht-

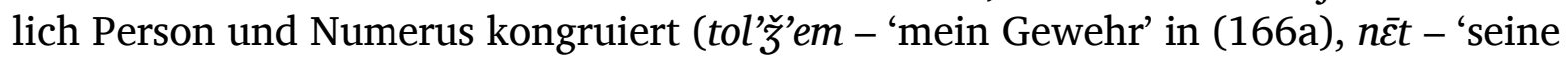
Tochter' in (166b)). Für Genitiv-NPs zum Ausdruck von Possessivität, wie sie in den Kopulasätzen belegt sind, finden sich keine Beispiele. 
a. $[M a-n a n]_{N P . L O C} f \bar{a}$ tol'̌̌̉e-m.

3SG-ADE good ski-1SG

'Ich habe gute Skier.'

(MNS_1984_BrotherSister_flk_44, Z: Narym)

b. $[\text { Tab-nan }]_{N P . L O C}$ nāgur n $\overline{\bar{\varepsilon}}$-t.

3SG-ADE three daughter-3SG

'Er hat drei Töchter.'

(SEV_1980_HazelGrouse_flk_17, S: Middle Ob)

\section{Zusammenfassung}

Zusammengefasst lässt sich festhalten, dass in verblosen Sätzen, wie in Kopulasätzen auch, unterschiedliche Relationen ausgedrückt werden. Verblose Sätze verfügen ebenfalls über zwei Komplemente, eine Kopula gibt es nicht. Die Objekt-Komplemente können aus unterschiedlichen Nominalphrasen (Nominativ-NP, Instrumental-NP, Lokativ-NP), Pronomina, Adjektiven, Partizipien oder Adverben bestehen. Während mit Nominativ-NPs die Identität des Subjektes ausgedrückt wird, stellen Komplemente in Form von Instrumental-NPs, Adjektiven, Partizipien und Adverben attributive Relationen zum Subjekt her. Wie in Kopulasätzen werden mit Lokativ-NPs lokale, existenzielle und possessive Relationen hergestellt.

\subsubsection{Unpersönliche Konstruktionen}

Unpersönliche Konstruktionen können formal-syntaktisch als Konstruktionen definiert werden, denen ein grammatisches (referentielles) Subjekt fehlt (Siewierska 2008, Malchukov/Ogawa 2011a: 20). In Anlehnung an Malchukov/Ogawa (2011a: 21f.) gehören hierzu auch Konstruktionen mit Pseudo-Subjekten sowie Konstruktionen, in denen dem grammatischen Subjekt (teilweise) spezifische Eigenschaften fehlen. Die Eigenschaften eines Subjektes umfassen verschiedene Bereiche wie „functional properties“, „semantic properties“, „coding properties“, „structural properties“und „behavioral properties“ (ebd.: 21f.). Im Rahmen der vorliegenden Arbeit wird der Fokus jedoch einschränkend auf die Relation zwischen funktionalen und formalen Eigenschaften gesetzt. Von den bei Malchukov/Ogawa (ebd.) unterschiedenen Typen lässt sich im selkupischen Textkorpus lediglich ein Typus von unpersönlichen Konstruktionen belegen:

- Fehlen eines referentiellen Subjekts (kovertes Subjekt bzw. Pseudo-Subjekt)

Konstruktionen mit indefiniten Subjekten lassen sich aufgrund des Pro-DropCharakters des Selkupischen (vgl. Kapitel 3) nicht sicher belegen. Als Argument kann hier Malchukovs Beobachtung angeführt werden, wonach „constructions with indefinite impersonal pronouns [...] have been shown to be common in non-pro-drop languages (ebd.: 28). Nicht-topikale Subjekte lassen sich im Korpus nicht nachweisen. Ei- 
nerseits fehlen notwendige Annotationen zum Informationsstatus, andererseits müssten derartige Konstruktionen mit muttersprachlichen Sprechern überpüft werden, die für die zentralen und südlichen Dialekte ebenfalls fehlen. Die beiden angeführten Typen, in denen das Subjekt unbelebt ist bzw nicht als Agens fungiert, sind im Korpus ebenfalls nicht belegt. Die Beispiele (167a) und (167b) zeigen allerdings mögliche Belege. Das grammatische Subjekt $m$ 'arga - 'Wind' ist mit dem Instrumental markiert und erinnert an Passivkonstruktionen im Russischen wie in Beispiel (168). Die selkupischen Verben erscheinen jedoch in beiden Beispielen im Aktivt. Aufgrund fehlender weiterer Beispiele lassen sich keine genaueren Aussagen treffen. Dieser Schritt sollte in künftigen Forschungen unternommen werden.

a. M'arg-ze tādì-r-ba-t kibajčc-p.

wind-INSTR bring-FRQ-PST-3SG.O boy-ACC

'Der Wind trug den Jungen fort.'

(SEV_1980_HazelGrouse_flk_47, S: Middle Ob)

b. Kōra-nd natidēli kỉb-andi-p čōčči-mba-t m'arg-zE.

sand-ILL there small-boat-ACC throw-PST.REP-3SG.O wind-INSTR

'Dort auf den Sand legte der Wind den Jungen.'

(SEV_1980_HazelGrouse_flk_27, S: Middle Ob)

(168) Vetr-om nes-lo mal'čik-a.

wind-INSTR carry-PST.3SG.NEUTR boy-ACC

'Der Wind trug den Jungen fort.'

(Russisch)

Malchukov unterscheidet verschiedene Untergruppen innerhalb der Gruppe der nicht-referenziellen Subjekte. Dazu gehören Pseudo-Subjekte, Null-Subjekte, Pseudo(Hilfs-)Verben sowie Kognate (ebd.: 24ff.). Im Selkupischen werden mit unpersönlichen Konstruktionen Kalenderphänome (Jahreszeiten, Tag, Nacht), Wetterphänomene (Regen, Schnee, Wind sowie Temperaturausdrücke wie Kälte, Wärme) ausgedrückt. Nicht in allen zentral- und südselkupischen Dialekten sind sämtliche Untergruppen belegt, teilweise sind überhaupt nur wenige Beispiele für die jeweiligen Phänomene im Korpus zu finden.

In beiden Dialektgruppen kann das Phänomen des Dunkelwerdens mit einer Konstruktion ausgedrückt werden, die ein Pseudo-(Hilfs-)Verb enthält. Im zentralselkupischen Beispiel (169a) ist es das Verb ed'e - 'werden', im südselkupischen Beispiel (169b) wird das Hilfsverb $e$ - 'sein' verwendet. Das Lexem 'dunkel' ist in beiden Beispielen das Adverb laba bzw. libi. Das formale Subjekt in Beispiel (169c) nop - 'Himmel' ist weder ein Pseudo-Subjekt noch ein lexikalisches Subjekt, es ist dazwischen angesiedelt (vgl. hierzu auch Malchukov/Ogawa (2011a: 26). Die finite Verbform ist abgeleitet vom Adjektiv lip - ‘dunkel'.

\footnotetext{
${ }^{4}$ Das Selkupische verfügt nicht über die Kategorie des Genus verbi.
} 
(169)

a. Kore-yit laba-ya- $\eta$ ed'e-mba, earth.house-LOC dark-DIM-ADV become-PST.REP.3SG tašsu-gu-mba. freeze-ITER-PST.REP.3SG

'In der Erdhütte ist es dunkel und kalt geworden.' (ChDN_1983_MistressOfFire_flk_28, Z: Vasjugan)

b. Māt-qit libit-yə-n $e-k$. house-LOC dark-DIM-ADV be-3SG.S 'Im Haus ist es dunkel.' (TET_1979_HowBeastsSearchedSun_flk_7, s: Middle Ket')

c. Nop tau lip-wa-t-pa. sky there dark-VBLZ-DRV-PST.REP.3SG

'Dort war es dunkel.'

(TMR_1967_Poenege_flk_22, S: Middle Ob)

Auch die Lexeme 'Tag' und 'Nacht' drücken Tageszeiten aus. Für das Lexem 'Nacht' sind vor allem Pseudo-(Hilfs-)Verb-Konstruktionen belegt wie in (170a) - (170c). Dabei können unterschiedliche Verben verwendet werden wie ē̌̌e und ēzu - 'werden' in (170a) und (170b) oder qwon - 'weggehen' in (170c). Die Konstruktion in (170d) enthält kein referentielles Subjekt, die finite Verbform pimna ist vom Nomen $p i-$ 'Nacht abgeleitet'.

a. Pe-n ē̌̌e-mba night-LOC.ADV become-PST.REP.3SG

'Es wurde Nacht.' (MNS_1984_DaughterOfEarth_flk_39, Z: Narym)

b. Pi-ta ēzu-ndi.

night-3SG become-IPFV.3SG

'Es wurde Nacht.'

(BNN_1971_DogSearchedMistress_flk_19, S: Middle Ket')

c. Pi-tta qwon-di.

night-3SG go.away-IPFV.3SG

'Es wurde Nacht.'

(BNN_1971_DogSearchedMistress_flk_31, S: Middle Ket')

d. Pi-m-nə

night-VBLZ-AOR.3SG

'Es wurde Nacht.'

(BNN_1971_DogSearchedMistress_flk_44, S: Middle Ket')

Für das Lexem 'Tag' sind im Korpus neben der oben schon beschriebenen Pseudo(Hilfs-)Verb-Konstruktion wie in (171c) auch Konstruktionen belegt, die Kognate enthalten wie čele/t'ēla - 'Sonne' in (171a) und (171b). In (171e) ist tēlì - 'Sonne' als 
verbalisierte Form in eine Konverbkonstruktion mit einem Phasenverb (kwatta - 'beginnen') eingebettet. In (171f) ist ebenfalls eine verbalisierte Form enthalten, hier als finite Verbform tēlimba - 'hell.sein'.

a. Čele-dì čelì-m-ba.

sun-3SG sun-TRL-PST.REP.3SG

'Die Sonne scheint.'

(ChDN_1983_ItjaStayedAlone_flk_25, Z: Narym)

b. T'ēla t'ēla-yz-ni.

sun sun-VBLZ-AOR.3SG

'Es wurde hell.'

(TET_1979_HowBeastsSearchedSun_flk_9, S: Middle Ket')

c. Čel-dì pat-pa.

sun-3SG go.down-PST.REP.3SG

'Die Sonne ist untergegangen.'

(MNN_1977_ItjaGrandmother_flk_12, Z: Tym)

d. Tēlit-t üüi-mba.

sun-3SG stop-PST.REP.3SG

'Die Sonne ist untergegangen.'

(TMR_1981_Robbers_flk_8, S: Middle Ob)

e. Tëli-q-le kwatta-lzi-mba.

sun-VBLZ-CVB begin-IPFV-PST.REP.3SG

'Es begann hell zu werden.'

(TMR_1981_Robbers_flk_38, S: Middle Ob)

f. Kuza-t sawsem tēli-m-ba

when-LOC.ADV at.all sun-VBLZ-PST.REP.3SG

'Als es ganz hell war, ...'

(SEV_1981_SisterBrother_flk_67, S: Middle Ob)

$\mathrm{Zu}$ Kalenderphänomenen gehören neben Ausdrücken für die Tageszeit auch die Jahreszeiten. Im Korpus werden jahreszeitliche Ausdrücke typischerweise in der Form 'im Frühling, im Sommer, ...' verwendet. Vereinzelt sind aber auch unpersönliche Konstruktionen wie in den südselkupischen Beispielen (172a) - (173) belegt. Ip allen Konstruktionen werden Pseudo-(Hilfs-)Verben verwendet, $\vec{e} d$ ' $a-$-'werden' in (172a), mèta - 'ankommen' in (172b) sowie tüw $w a$ - 'kommen' in (173).
a. $\overline{\tilde{U}} d a-t \quad \bar{e} d^{\prime} a$.
spring-3sg become.3sG
'Es wird Frühling.'
(TET_1979_SnowGirl_flk_12, S: Middle Ket')

b. $\bar{\partial} d \partial-t \quad m e \bar{t} a$.

spring-3sg achieve.3sG 
'Es wird Frühling.'

(TFF_1967_KolobokkaPoenegessa_flk_4, S: Middle Ob)
(173)
Tü-wa
$q \dot{i}-t$
$n \bar{u}$.
come-AOR.3SG winter-3SG now
'Es wurde nun Winter.'
(TET_1979_TheHaresHouse_flk_2, S: Middle Ket')

$\mathrm{Zu}$ den Wetterphänomenen gehören auch Temperaturausdrücke, die ebenfalls mit unpersönlichen Konstruktionen gebildet werden, allerdings sind nur einzelne Beispiele im Textkorpus belegt. In (174) erscheint taše - 'gefrieren' als lexikalisches Verb. Die Beispiele (175a) und (175b) enthalten das Lexem 'regnen', in (175a) als lexikalisches Verb, in (175b) als Pseudo-Subjekt mit čaža - 'gehen' als finiter Verbform. Das Lexem serro drückt sowohl 'Regen' als auch 'Schnee' aus. Im zentralselkupischen Beispiel (176) findet sich mit t'ōwa ein weiteres Lexem für 'schneien'. Es erscheint wie alle unpersönlichen Konstruktionen in der 3. Person Singular.

(174) Uruk taše-ha.

very freeze-AOR.3SG

'Es war sehr kalt.'

(ChDN_1983_MotherInLaw_nar_7, Z: Vasjugan)

$$
\begin{aligned}
& \text { a. ... aqqol serro- } n \text { ' } i \\
& \text { again rain-VBLZ.3sG }
\end{aligned}
$$

'... wieder regnet es.' (KKN_1971_IvanAndGod_flk_12, S: Upper Ket')

b. Serro-t čaร̌a- $\eta$ rain-3SG go-3SG.S

'Es regnet.'

(KKN_1971_IvanAndGod_flk_6, S: Upper Ket')

$$
\begin{aligned}
& \text {... merge-n-ni் i t'ōwa-lži- } \eta \text {. } \\
& \text { wind-VBLZ-AOR.3sG and snow-IPFV-3sG.s } \\
& \text { '... kam Wind auf und es begann zu schneien.' } \\
& \text { (KMS_1966_TwoSisters_flk_9, S: Middle Ket') }
\end{aligned}
$$

Ebenfalls zu Wetterphänomen zählen Konstruktionen, in denen das Lexem 'Wind' verwendet wird. In Beispiel (177a) sind Kognate enthalten, ähnlich wie in den Beispielen (171a) und (171b) oben. Das Lexem m'arg - 'Wind' erscheint dort als Nomen und als derivierte Verbform in der 3. Person Singular. Der Konstruktion in (177b) fehlt ein referentielles Subjekt, die Verbform ist wiederum abgeleitet.

a. A m'arg m'arge- $n-n i$.

but wind wind-VBLZ-AOR.3sG

'Und der Wind blies.'

(SEV_1981_SisterBrother_flk_23, S: Middle Ob) 


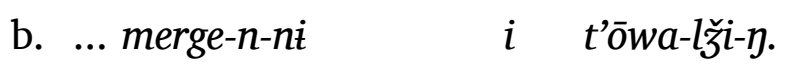

wind-VBLZ-AOR.3SG and snow-INCH-IPFV-3SG.S

'... kam Wind auf und es begann zu schneien.'

(KMS_1966_TwoSisters_flk_9, S: Middle Ket')

Das Subjekt nom - 'Himmel' in der unpersönlichen Konstruktion in (178a) ist zwischen einem Pseudo-Subjekt und einem lexikalischen Subjekt. Eine analoge Konstruktionen findet sich in (169c) oben und im arabischen Beispiel (178b).
a. Warzq mad'o-yəntu nom merk'e-n-ni-n wārqi- $\eta$.
big taiga-ABL.3sG sky wind-VBLZ-AOR-3sG.s big-ADV
'Aus der großen Taiga wehte ein starker Wind.'
(PMP_1961_ForestWoman_flk_294, S: Middle Ob)
b. id-dunya ti-shti.
the-world 3sG.MS-rain
'Die Welt regnet.'
(Malchukov/Ogawa 2011a: 27, Arabisch)

Zusammenfassend lässt sich beobachten, dass das Selkupische unterschiedliche Strategien verwendet, um unpersönliche Konstruktionen auszudrücken. Im Korpus sind Konstruktionen mit Kalenderphänomen und Wetterphänomenen belegt. Neben fehlenden referentiellen Subjekten (mit abgeleiteter finiter Verbform) können KognatKonstruktionen, Pseudo-(Hilfs-)Verb-Konstruktionen sowie eine Konverbkonstruktion belegt werden. Eine weitere Möglichkeit sind Subjekte, die 'Welt', 'Wetter' oder 'Himmel' bezeichnen und zwischen lexikalischen Subjekten und Pseudo-Subjekten angesiedelt werden Malchukov/Ogawa (2011a: 26f.) wie in den Beispielen (169c) und (178a). In beiden Beispielen wird das Lexem für 'Himmel' in den Varianten nop und nom verwendet 5 . Derartige Beispiele sind ebenso wie die Konverbverbindungen ausschließlich für das südselkupische Teilkorpus belegt.

Unpersönliche Konstruktionen erscheinen im Korpus nur in der 3. Person Singular. Unpersönliche Translativ-Konstruktionen, wie sie Malchukov/Ogawa (2011b: 406) beschreibt, sind im Korpus nicht belegt. Auch der von ihr postulierte Aorist als grundsätzliches Tempus lässt sich mit den Korpusdaten nur bedingt bestätigen. Neben Aorist-Formen wie in (170d), (171b), (174), (\{176), (177a) und (178a) sind auch Formen im Präteritum wie in (169c) sowie reportativem Präteritum wie in (171a), (171f) belegt.

Pseudo-Subjekte in unpersönlichen Konstruktionen können mit dem Possessivsuffix der 3. Person Singular markiert sein. Im Korpus belegt sind serro - 'Regen' (Beispiel (175b)), qi - 'Winter' (Beispiel (173)), ādz - 'Frühling' (Beispiele (172a) und (172b)), cele/t'ela - 'Sonne' (Beispiele (171a), (171c), (171d)) und pi - 'Nacht' (Beispiele (170b) und (170c)). Tabelle 18 fasst die unpersönlichen Satztypen zusammen.

${ }^{5}$ Ein ähnliches Beispiel mit nom findet sich auch bei Malchukov/Ogawa (2011b: 413), hier in der Übersetzung 'Wetter'. 
Tab. 18: Unpersönliche Konstruktionen im Selkupischen

\begin{tabular}{lll}
\hline & Zentralselkupisch & Südselkupisch \\
\hline Verb $_{\text {FIN }}+$ Null-Subjekt & $\mathrm{X}$ & $\mathrm{X}$ \\
Kognate & $\mathrm{X}$ & $\mathrm{X}$ \\
Pseudo-(Hilfs-)Verb & $\mathrm{X}$ & $\mathrm{X}$ \\
'Himmel' als Subjekt & & $\mathrm{X}$ \\
CVB-Konstruktion & & $\mathrm{X}$ \\
\hline
\end{tabular}

\subsection{Interrogativsätze}

Im Selkupischen lassen sich polare Interrogativsätze von sogenannten wInterrogativsätzen unterscheiden. Mit ersteren werden Entscheidungsfragen ausgedrückt, letztere werden mittels Interrogativwörtern eingeleitet. Dir Wortfolge ist in beiden Subtypen variabel, wobei in w-Interrogativsätzen das Interrogativwort in der Regel satzinitial erscheint.

\subsection{1 w-Interrogativsätze}

Ergänzungsfragen, in denen ein Sachverhalt unter einem bestimmten Aspekt in Frage gestellt wird, werden im Selkupischen mit Interrogativwörtern eingeleitet. Es lassen sich hierbei Interrogativwörter (vgl. Tabelle 28), die sich formal wie Nomina verhalten (Numerus- und Kasussuffixe annehmen können) von solchen unterscheiden, die sich mehr wie Adverben verhalten. Zur ersten Kategorie gehören die Interrogativwörter qaj - 'was', kutì - 'wer', ku - 'wo', kūča - 'wann, wohin', kušša - 'wieviel' und kul'di - 'welcher', letzteres ist ausschließlich im südselkupischen Ket'-Dialekt belegt. Zur zweiten Kategorie gehören die Interrogativwörter qanduk 'wie', quttar - 'wie' und qayä - 'wann', wobei letzteres ebenfalls ausschließlich im südselkupischen Ket'-Dialekt belegt ist. Die von Bekker (1995b: 100ff.) beschriebenen vollständigen Numerus- und Kasusparadigmen lassen sich im Belegkorpus nicht mehr nachweisen. So ist beispielsweise keine Dualmarkierung mehr belegt. In der Regel erscheinen die Interrogativwörter in Interrogativsätzen satzinitial, es sind jedoch auch andere Wortstellungen möglich. Die nachfolgenden Beispiele zeigen die belegten Interrogativwörter in beiden Dialektgruppen.

\section{qaj}

Das Interrogativpronomen qaj - 'was' kann Kasussuffixe tragen, belegt sind Lokativ ('wo'), Translativ ('warum'), Prolativ ('woher'), Instrumental ('womit, worüber'), Akkusativ ('wen') und Ablativ ('woher'). Mit dem Adjektivierer -l markiert formt es die Bedeutung 'was für eine Art bzw. welcher'. In den Beispielen (179a) und (179b) erscheint das Pronomen unmarkiert und satzinitial oder ist eingeschoben in eine Lokativkonstruktion. Die Beispielsätze (179c) und (04:INTERROG:04) zeigen die kasusmarkierten Varianten 'woher' und 'warum'. 
(179)

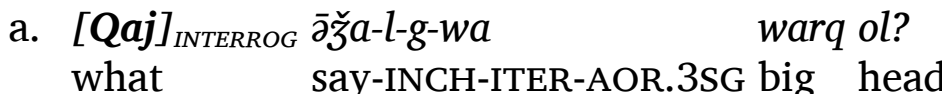
'Was hat der König gesagt?'
(SEV_1980_HazelGrouse_flk_51, S: Middle Ob)
b. Tan-nan [qaj] INTERROG $n a t^{\prime} e-\gamma$ in ugulže-yin
2SG-ADE what there-LOC.ADV home-LOC.ADV
ed'e-mba?
become-PST.REP.3SG
'Was ist dort bei dir zu Hause passiert?'
(ChDN_1983_MistressOfFire_flk_48, Z: Vasjugan)
c. ... [Kaj-mute $]_{\text {INTERROG }}$ nika to-lla hurup?
what-PROL here come-OPT.3sG wild.animal
'Woher sollte hier ein Bär (wrtl.: wildes Tier) kommen?'
(ChDN_1983_BearCameIntoVillage_nar_16, Z: Vasjugan)
d. Tan [qai-no] $]_{\text {INTERROG }}$ mazim ora-l-ma-to?
2SG what-TRL 1SG.ACC hold-INCH-HAB-2SG.S
'Warum hältst du mich?'
(PVD_1966_BoyDevil_flk_10, S: Chaja)

\section{kuti}

Das Interrogativpronomen kuti - 'wer' erscheint im Belegkorpus in verschiedenen Varianten, die Beispiele zeigen die Varianten kud, kod,kuda und kudo. Das Interrogativpronomen tritt zumeist unmarkiert auf, wie in (180a) und (180b), kann jedoch auch Kasussuffixe tragen, wie in (180c). Beispielsatz (180d) zeigt eine verblose Konstruktion, das Interrogativpronomen trägt hier die Verbalendung der 2. Person Singular $(-n t)$. Ähnlich wie bei qaj, erscheint kudi in der Regel satzinitial, andere Positionen wie in (180d) sind jedoch möglich.
a. Tat tenu-a-l
mat $[\mathrm{kud}]_{\text {INTERROG }} e-n d a-k$ ?
2SG know-AOR-2SG.O 1SG who
be-INFER-1SG.S
'Weisst du, wer ich bin?'
(TTD_1964_CalfHare_flk_25, Z: Tym)
b. $[\text { Kod }]_{\text {INTERROG }}$ tö-na-nž?
who come-AOR-2sG.S
'Wer kommt?'
(TMR_1981_AboutItja_flk_14, S: Middle Ob)
$\begin{array}{ll}\text { c. }[\text { Kuda-se }]_{\text {INTERROG }} & \text { kuan-ža-k? } \\ \text { who-COM } & \text { go.away-FUT-1SG.S }\end{array}$
'Mit wem werden ich gehen?'
(NN_1855_Hero1_song_5, Z/S: Tym/Middle Ob)
d. Tat [kudo-nt?] $]_{\text {INTERROG }}$
2SG who-2SG.S 
'Wer bist du?'

(ChDN_1983_TaleBrownies_flk_18, Z: Vasjugan)

ku

Das Interrogativpronomen $k u$ - 'wo, wohin' ist in Interrogativsätzen fast nur in unmarkierten Varianten belegt, wie in (180a) und (180a). Beispiel (180a) zeigt $k u$ in der Bedeutung 'wo', es ist mit einem Adverbialkasus ( $-n)$ markiert. Hinsichtlich der Wortstellung verhält es sich wie qaj und kutt.

a. $[\mathbf{K u}]_{\text {INTERROG }}$ mazik tade-r-a-d?

where 1SG.ACC bring-FRQ-EP-3SG.O

'Wohin bringt es mich?'

(ChDN_1983_GirlAndIce_flk_12, Z: Vasjugan)

b. $\mathrm{Nu}, \quad[\mathrm{ku}]_{\text {INTERROG }} k a d \bar{\varepsilon}-n \check{\zeta} a-k$ ?

INTERJ where stay-FUT-1SG.S

'Nun, wo soll ich bleiben?'

(NN_NN_IWentFishing_nar_11, S: Middle Ob)

c. Kučer-o-l [ku-n $]_{\text {INTERROG }} \quad e-j j-a$ ?

coachman-EP-2SG where-LOC.ADV be-AOR.3SG

'Wo ist dein Kutscher?'

(SDP_1964_FairytaleBlackZar_trans_322, Z: Narym)

kūěa

Die Form kūča wird im Textkorpus mit lokaler Bedeutung 'wohin' und mit temporaler Bedeutung 'wann' in Interrogativsätzen verwendet. kūča ist nur mit Lokalkasus (Lokativ, Ablativ) wie in Beispiel (182c) sowie dem Adverbialkasus - $n$ /-t (Beispiele (182a), (182b) und (182d)) belegt. Die Wortstellung ist variabel.

(182) a. Mat temn'a-m [quč'a-n] $]_{\text {INTERROG }} e-j j-a$ ?

1SG brother-1SG where-LOC.ADV be-AOR.3SG

'Wo ist mein Bruder?'

(SDP_1964_FairytaleBlackZar_trans_973, Z: Narym)

b. Mama, eu-n-t tamd'el am-z-ut, a nē-la-n-t

mum mother-ACC-3SG today eat-FUT-1PL but daughter-PL-ACC-3SG

[kuza-n] $]_{\text {INTERROG }}$ ?

when-LOC.ADV

'Mama, die Mutter werden wir heute essen, aber wann die Töchter?'

(PMP_1961_ForestWoman_flk_89, S: Middle Ob)

c. Tat [kuča-gandi $]_{\text {INTERROG }}$ töu-a-nd?

2SG where-ABL.3SG come-AOR-2SG.S

'Woher bist du gekommen?'

(NN_1855_Hero3_song_11, Z/S: Tym/Middle Ob)

d. [Kuča-d $]_{\text {INTERROG }}$ kuan-ne-ň̌e-t?

where-LOC.ADV go.away-DRV-FUT-3SG.O 
'Wohin wird man sie bringen?'

(NN_1855_Hero1_song_149, Z/S: Tym/Middle Ob)

\section{kušša}

Das Interrogativpronomen kušša - 'wieviel' ist im Textkorpus ausschließlich mit den Derivationssuffixen $-l /-j$ (Adjektivierer) bzw. $-n /-k$ (Adverbmarker) belegt. In Interrogativsätzen lässt sich seine Verwendung in nur einem Beispiel nachweisen.

(183) Hm, Hm, [kušša-j] $]_{\text {INTERROG }}$ qomde-t?

INTERJ INTERJ how.many-ADJZ money-3SG

'Hm, hm, wieviel Geld?'

(PMP_1961_Fairytale_flk_196, S: Middle Ob)

\section{kul'di}

Das Interrogativwort kul'di ist ausschließlich im südselkupischen Ket'-Subkorpus belegt. Seine Verwendung in Interrogativsätzen beschränkt sich auf das folgende Beispiel (184), das zudem eine russische Syntax aufweist (russ. kakoe delo sdelat' tebe 'Welche Sache (soll ich) für dich (wrtl.: dir) tun?').

(184) [Kul'di $]_{\text {INTERROG }}$ dela-m me-gu tendani? which thing-ACC do-INF 2SG.DAT

'Welche Sache (soll ich) für dich tun?'

(AGS_1968_FairytaleSnake_flk_57, S: Middle Ket')

\section{qundar}

Nur in südselkupischen Dialekten belegt ist qundar - 'wie'. Außer in Interrogativsätzen wird es auch in komparativen Konstruktionen verwendet wie in Beispiel (185). Die Beispiele (186a) - (186c) zeigen die Verwendung als Interrogativpronomen.

(185) Süni-m-žă-lat n'uzว-qanž-la, [qundar] $]_{\text {INTERROG }}$ aw qanža-la. unload-DRV-IPFV-IMP.2PL grass-sledge-PL how this sledge-PL

'Heuschlitten, entladet euch, wie diese Schlitten!' (PMP_1966_BodylessHead_flk_51, S: Middle Ob)
$k e-t$ meja, ask-IPFV-CVB begin-FRQ-EP-3SG.S say-IMP.2SG.O 1SG.DAT
a. Sōqu-nžu-l'e übə-r-a-n:
[kundar $]_{\text {INTERROG }}$ sü t'äri- $\eta$ ?
how dragon say-3SG.S
'Er begann zu fragen: Sag mir, wie spricht der Drache?' (PMP_1961_Fairytale_flk_283, S: Middle Ob)
b. A eze-n-to nim [kundar $]_{\text {INTERROG }}$ je-wa-n? and father-GEN-3SG name how be-AOR-3SG.S
'Und wie ist der Name des Vaters?' (PMP_1966_BoyDevil_flk_50, S: Middle Ob)

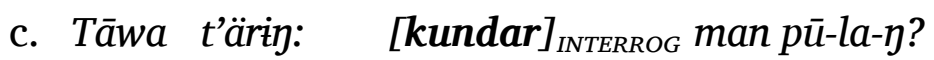 mouse say-3SG.s how 1SG cross-OPT-1SG.S 
'Die Maus sagt: Wie komme ich hinüber?'

(KMS_1963_HazelgrouseMouse_flk_25, S: Middle Ket')

\subsubsection{Polare Interrogativsätze}

Entscheidungsfragen werden mit polaren Interrogativsätzen (auch Ja-/Nein-Fragen) ausgedrückt. Dabei wird ein Sachverhalt als Ganzes in Frage gestellt. Sie verlangen keine spezielle Wortfolge, das finite Verb kann satzfinal erscheinen, wie in (187a) und (187b), andere Stellungen sind jedoch auch möglich, wie in (187c). Polare Interrogativsätze sind vor allem durch den Kontext identifizierbar. Aufgrund der fehlenden formalen Unterscheidung müsste untersucht werden, inwiefern die Intonation eine weitere relevante Größe darstellt. Dieser Aspekt überschreitet jedoch den Rahmen der vorliegenden Arbeit bei weitem, zudem fehlen im zugrundeliegenden Textkorpus Audiosprachdaten.

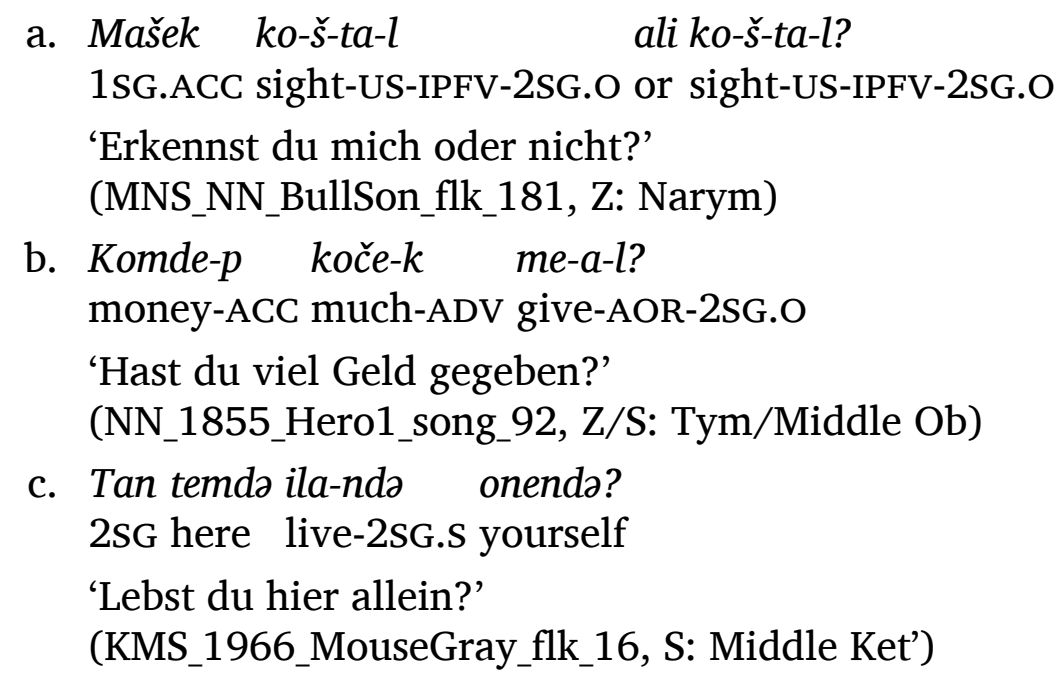

Es kann zusammengefasst werden, dass sich im Selkupischen zwei Typen von Interrogativsätzen unterscheiden lassen: Mit den sog. w-Interrogativsätze werden Ergänzungsfragen formuliert, in denen ein Sachverhalt unter einem bestimmten Aspekt in Frage gestellt wird. Sie werden mit Interrogativwörtern eingeleitet, die in den Beispielen (179a) - (186c) dargestellt sind. Dabei verhalten sich qaj - 'was', kuti - 'wer', $k u$ - 'wo', kūcra - 'wann, wohin', kušša - 'wieviel' und kul'di - 'welcher' formal eher wie Nomen (sie können Numerus- und Kasussuffixe annehmen), qanduk - 'wie', quttar 'wie' und qayä - 'wann' eher wie Adverben. Die ursprünglich vorhandenen vollständigen Kasus- und Numerusparadigmen der Interrogativwörter sind im Textkorpus nur noch bruchstückhaft belegt.

Mit polaren Interrogativsätzen werden Entscheidungsfragen ausgedrückt. Sie verfügen über kein einleitendes Interrogativwort. Auch die Wortfolge kann variieren, wie in den Beispielsätzen (187a) - (187c) gezeigt ist. Polare Interrogativsätze werden vor allem durch den Kontext identifiziert. Weitergehende Untersuchungen zur Unterscheidung von Deklarativsätzen und polaren Interrogativsätzen fehlen bisher. Insbe- 
sondere die Intonation als Unterscheidungsmöglichkeit lässt sich aufgrund des fehlenden Audiomaterials im zugrundeliegendem Textkopus nicht betrachten.

\subsection{Imperativsätze}

Der Imperativ weist im Selkupischen in beiden Konjugationsformen (subjektiv und objektiv) in allen Personen und Numeri eigene Personalendungen auf. Im Korpus kommen Formen der 2. Person am häufigsten vor. Ebenso wie in Deklarativsätzen lassen sich auch in Imperativkonstruktionen beide Konjugationsarten sowohl mit unmarkierten als auch mit Akkusativobjekten finden (vgl. auch Bekker (1995b: 106, 141) und Wickman (1955)). Die folgenden Beispiele zeigen Imperativsätze in beiden Dialektgruppen.

Imperativsätze, in denen die zweite Person Singular mit der objektiven Konjugation des Verbs erscheint, kommen in beiden Dialektgruppen sowohl mit unmarkierten Objekten wie in (188a) und (188b) als auch mit Akkusativobjekten wie in (189a) und (189b) vor. Das Subjekt ist in der Regel kovert.

(188) Zweite Person Singular objektiv, Nominativobjekt

a. $[\bar{I}-l]_{\text {NOM }}$ m'e-d! son.NOM-2SG give-IMP.2SG.O

'Gib (mir) deinen Sohn!'

(ChDN_1983_MistressOfFire_flk_74, Z: Vasjugan)

b. [Segi-llaga $]_{N O M}$ t'az-et.

ball.NOM-SING throw-IMP.2SG.O

'Wirf das Knäuel!'

(PMP_1961_ForestWoman_flk_39, S: Middle Ob)

(189) Zweite Person Singular objektiv, Akkusativobjekt

a. Čačč $-d$ [mašik $]_{A C C} \varepsilon t-t$, mat tēka prigazūs'.

throw-IMP.2SG.O 1SG.ACC water-ILL 1SG 2SG.DAT be.of.use.1SG

'Wirf mich ins Wasser, ich werde dir nützlich sein!'

(TTD_1964_Frog_flk_101, Z: Tym)

b. Tan kur-o-l-ga, tat-to mega [salsabü-m] $]_{A C C}$ !

2SG run-EP-INCH-AOR.3SG bring-IMP.2SG.O 1SG.DAT grinder-ACC

'Du lauf, bring mir den Schleifstein!'

(PVD_1966_BoyDevil_flk_34, S: Chaja)

In Imperativsätzen, in denen die zweite Person Singular mit der subjektiven Konjugation des Verbs erscheint, zeigen die südselkupischen Dialekte neben Akkusativobjekten wie in (191b) auch direkte Objekte im Nominativ, wie in (190a) und (190b). Für das Zentralsselkupische sind nur Beispiele mit Akkusativobjekt wie in (191a) belegt. 
(190) Zweite Person Singular subjektiv, Nominativobjekt

a. Tan mey [noppi $]_{\text {NOM }} m \bar{e}-g a-q$ !

2SG 1SG.DAT glove.NOM do-ITER-IMP.2SG.S

'Du mach mir Handschuhe!'

(KMS_1966_TwoSisters_flk_31, S: Middle Ket')

b. Selna-j [wedra] $]_{\text {NOM }}$ saq tulža-q!

whole-ADJZ bucket.NOM salt bring-IMP.2SG.S

'Bring einen Eimer voll Salz!'

(PVD_1961_FarmAssault_flk_109, S: Upper Ob)

(191) Zweite Person Singular subjektiv, Akkusativobjekt

a. M'izenit $[t \overline{\ddot{u}}-m]_{A C C} m$ 'e-je-š!

1PL.DAT fire-ACC give-AOR-IMP.2sG.S

'Gib uns Feuer!'

(ChDN_1983_MistressOfFire_flk_66, Z: Vasjugan)

b. It'e-ne t'ara- $\eta$ : s'im $_{\text {ACC }}$ sappo-zi-q!

Itja-DAT say-3SG.S 1sG.ACC spring.out-DRV-IMP.2sG.S

'Er sagt zu Itja: Zieh mich raus!'

(KKN_1971_Itja_flk_19, S: Upper Ket')

Die 1. und 2. Person Dual sowie die 2. Plural sind im Textkorpus ausschließlich mit dem Akkusativ markiert, die entsprechenden Beispiele (192a) und (192b) - 1 . Person Dual, (193a) - 2. Person Dual und (193b) - 2. Person Plural stammen aus dem südselkupischen Subkorpus. Zentralselkupische Beispiele gibt es gar nicht.

(192) Erste Person Dual, Akkusativobjekt

a. Tan [ulo-m] $]_{A C C}$ mannu-mp-laj.

2sG head-ACC look-hab-IMP.1DU

'Lass uns deinen Kopf anschauen!'

(PMP_1961_ForestWoman_flk_62, S: Middle Ob)

b. Peke-ka t'äri- $\eta \quad p \bar{u}-l o \quad[k \dot{i}-k e-m]_{A C C} \quad \ldots$

hazel.grouse-DIM say-3SG.S cross-IMP.1DU river-DIM-ACC

'Das Haselhuhn sagte: Lass uns den Fluss überqueren, ...'

(KKA_NN_HazelGrouse_flk_7, S: Upper Ket')

(193) Zweite Person Dual und Plural, Akkusativobjekt

a. Na pēge àža-l-g-wa aze-qind,

this hazel.grouze say-INCH-ITER.3SG father-DAT/ALL.3SG

awe-qind: $\quad[\text { mašep }]_{A C C}$ nād-li!

mother-DAT/ALL.3SG 1SG.ACC get.married-IMP.2DU

'Dieses Haselhuhn sagte zu seinem Vater und seiner Mutter: Verheiratet mich!'

(SEV_1980_HazelGrouse_flk_14, S: Middle Ob) 
b. ... [İt'a-m] $]_{A C C} t \bar{i}$ tan-na-lat, lōs-ə-la!

Itja-ACC here bring-AOR-IMP.2PL devil-PL

'Bringt Itja hierher, Geister!'

(NN_1879_Itja_flk_66, S: Chaja)

Für die südselkupischen Dialekte sind Imperativsätze in der dritten Person Singular belegt. Beide Beispiele (194a) und (194b) enthalten intransitive Verben, so dass es kein direktes Objekt gibt. In (194b) ist das Subjekt das Interrogativpronomen qaj'was'. Zentralselkupische Beispiele lassen sich nicht belegen.

(194) Dritte Person Singular
a. Tau šakku-j-kwes
örim-ni
kwēz-i $[t a ̄ u-l a ̈]_{N O M} !$
this fire.iron-ADJZ-iron grow.up-IMP.3sG.s iron-ADJZ hill-DIM
'Dieser Feuerstein wachse zu einem eisernen Berg!'
(TFF_1967_ItjaAldigaPönegesse_flk_77, S: Middle Ob)
b. Täj [qaj] $]_{\text {NOM }}$ ti-ll-ej ti-ll-ej!
'Jenes Etwas möge herfliegen!'
(SVG_1964_IitekaPineweldju_flk_4, S: Upper Ket')
that what fly-INCH-IMP.3sG.s fly-INCH-IMP.3sG.S

\subsection{Negation einfacher Sätze}

Der einfache affirmative Satz (Kapitel 4 oben) kann mit Hilfe von Negationspartikeln

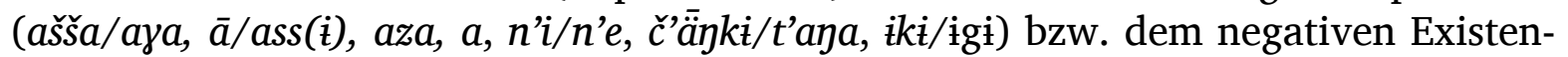
zialverb (čä́nki/ čang(w)a, t'ang(w)a) sowie dem negativen Hilfsverb (tača/ čed'a, čeža/ted'a - 'nicht können') negiert werden. Die folgenden Abschnitte 4.4.1 - 4.4.5 beschreiben negierte Deklarativ-, Interrogativ- und Imperativsätze.

\subsubsection{Negierter Deklarativsatz}

Deklarativsätze können mit Hilfe der Negationspartikel ašša bzw. ihren Varianten sowie mit dem negativen Existenzialverb č negiert werden (vgl. hierzu auch das Kapitel zu negierten Verbphrasen, 84 sowie Tabelle 14 zu den verwendeten Negationswörtern.). Wie in affirmativen Sätzen auch, kann das Subjekt (S) bzw. das Agens (A) sowohl in intransitiven als auch in transitiven Sätzen lexikalisch overt ausgedrückt sein. Gleiches gilt für das Patiens (P) (in monotransitiven Sätzen) bzw. Rezipienten (R) und Themata (T). Im Gegensatz zu affirmativen Sätzen, in denen Nominativ-Objekte häufig verwendet werden, sind sie für negierte Sätze im Korpus nur vereinzelt belegt, wie in (195). In ditransitiven Konstruktionen kommen sie nicht vor.

$$
\begin{array}{ll}
\ldots[\check{s} \bar{t} w]_{P . N O M} & \overline{\boldsymbol{a}} \quad \text { kotta-l-b-ni-m } \\
\text { ashes NEG throw-INCH-HAB-AOR-1SG.O if }
\end{array}
$$


'... hätte ich die Asche nicht weggeworfen.'

(MNS_1984_BrotherSister_flk_78, Z: Narym)

\subsubsection{Negierter intransitiver Deklarativsatz}

Ein affirmativer intransitiver Deklarativsatz kann mit Hilfe der Negationspartikel $a \check{s ̌ s} a$ bzw. ihrer Varianten negiert werden. Die Negationspartikel wird der finiten Verbform unmittelbar vorangestellt. Das nordselkupische Beispiel (196a) enthält eine Phasenverb-Konstruktion, bei der das Phasenverb olam - 'beginnen' mit der Infinitivform des lexikalischen Verbs qonti - 'schlafen' verschmolzen ist. Wie im affirmativen Pendant kann das Subjekt overt ausgedrückt werden wie in (196b) und (196c), muss es aber nicht wie in (196a)

a. Ǎša qonti-q-olam-na.

NEG sleep-INF-be.going.to-CO.3SG

'Er schläft nicht ein.'

(AAI_1973_Okyle_flk_35, N: Middle Taz)

b. N'edek čwesse aya manni-mba.

girl backward(s) NEG look-PST.REP.3SG

'Das Mädchen sah nicht zurück.'

(ChDN_1983_GirlAndIce_flk_23, Z: Vasjugan)

c. Tep-i-la-n $n \bar{e}-d \quad$ qaj-m-naja a kid-i-ku-d.

3SG-EP-PL-GEN daughter-3SG what-ACC = EMPH NEG say-EP-HAB-3SG.O

'Ihre Tochter sagte nichts.'

(TET_1979_SnowGirl_flk_15, S: Middle Ket')

\subsubsection{Negierter monotransitiver Deklarativsatz}

Ebenso wie ein intransitiver Satz kann ein affirmativer monotransitiver Satz mit Hilfe der vorangestellten Negationspartikel ašša bzw. eine ihrer Varianten negiert werden, wie in den Beispielen (197a) - (197c) gezeigt ist. Beispiel (197a) enthält ein nominales Akkusativpobjekt (ïjam - 'Kind.ACC'), Beispiel (197b) ein pronominales Akkusativobjekt (řıntì - '2SG.ACC', tabdip - '3PL.ACC'), Beispiel (197c) ein nominales unmarkiertes Objekt ( ̌̌šw - 'Asche').

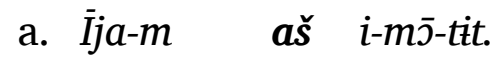
child-ACC NEG take-DRV-3PL

'Sie haben das Kind nicht genommen.'

(AAI_1973_Okyle_flk_35, N: Middle Taz)

b. $\left[M^{\prime} i\right]_{A}[t a b-d-\dot{i}-p]_{P . A C C}$ aya ko-nze-r-na-ut.

1PL 3SG-PL-EP-ACC NEG sight-IPFV-FRQ-AOR-1PL

'Wir sehen sie nicht.'

(ChDN_1983_TaleBrownies_flk_1, Z: Vasjugan) 


$$
\begin{aligned}
& \text { c. ... }[\check{s} \bar{t} w]_{\text {P.NOM }} \overline{\boldsymbol{a}} \text { kotta-l-b-ni-m bi. } \\
& \text {... ashes NEG throw-INCH-HAB-AOR-1SG.O if } \\
& \text { '... hätte ich die Asche nicht weggeworfen.' } \\
& \text { (MNS_1984_BrotherSister_flk_78, Z: Narym) }
\end{aligned}
$$

\subsubsection{Negierter ditransitiver Satz}

Ditransitive Sätze werden wie monotransitive Sätze negiert (siehe oben). Die Negationspartikel erscheint unmittelbar vor der finiten Verbform. Auch in negierten ditransitiven Sätzen können Agens, Patiens und Thema lexikalisch overt ausgedrückt werden wie in Beispiel (198c). Einzelne semantische Rollen können auch kovert realisiert sein, wie das Thema in (198a) oder das Agens in (198b). Negierte benefaktive Konstruktionen sind im Korpus nicht belegt.

$$
\begin{aligned}
& \text { a. }[M a t]_{A}[\text { täntinik }]_{R} \text { ašša mi-nta-p, } \quad \ldots \\
& \text { 1SG 2SG.DAT NEG give-FUT-1SG.O } \\
& \text { 'Ich werde (sie) dir nicht geben ...' } \\
& \text { (AVA_1973_Ichakicha2_flk_134, N: Middle Taz) } \\
& \text { b. }[T \text { 'ekka }]_{R}[p \bar{o}-m]_{T} \text { aya m'e-l'e-b'e. } \\
& \text { 2SG.DAT tree-ACC NEG give-FUT-1SG.O } \\
& \text { 'Ich werde dir das Holz nicht geben.' } \\
& \text { (ChDN_1983_MistressOfFire_flk_21, Z: Vasjugan) } \\
& \text { c. }[M a t]_{A}[t \bar{e} k a]_{R}[n \bar{\varepsilon}-p]_{T} \quad \text { qum-ne aza me-nža-p. } \\
& \text { 1SG 2SG.DAT daughter-ACC person-DAT/ALL NEG give-FUT-1SG.O } \\
& \text { 'Ich werde dir die Tochter nicht zur Frau geben.' } \\
& \text { (ILP_1981_ItjaOneEyedDevil_flk_14, S: Middle Ob) }
\end{aligned}
$$

Werden Interrogativpronomina verwendet, um Subjekt, Objekt oder Adverbiale zu negieren, unterscheiden sich die nordselkupischen Dialekte von den zentral- und südselkupischen. In ersteren wird eine Doppelnegation verwendet, indem das Interrogativpronomen mit der Negationspartikel $n ’ i$ und das Prädikat zusätzlich mit der Standard-Negationspartikel ašša (bzw. deren Varianten) negiert ist wie in den Beispielsätzen (199a) - (199b). Die Strategie wird in allen uralischen Sprachen und auch im Russischen verwendet ( $J a$ nikogo ne videla - 'Ich habe niemanden (nicht) gesehen.').

In den zentralen und südlichen Dialekten wird stattdessen an das Interrogativpronomen die emphatische Partikel naj angeschlossen, die Negation des Prädikats mit der Standardnegationspartikel ašša erfolgt ebenfalls, wie in den Beispielen (200a) (200g). Teilweise ist die emphatische Partikel mit dem Interrogativpronomen verschmolzen und daher verkürzt, wie in (200d). Trägt das Interrogativpronomen Kasussuffixe, wie in (200c) - (200e) und (200g), erscheinen diese vor der emphatischen Partikel. In (200f) ist zwischen dem Interrogativpronomen und dem negierten Prädikat ein weiteres Argument (ad'ukandennan - 'von der Großmutter') eingeschoben. 
(199) a. ... [n'i kuti̇] $]_{S}$ ašša lanki-n'-n'a.

NEG who NEG cry-US-AOR.3SG

'... niemand schreit.'

(AAI_1973_Okyle_flk_28, N: Taz)

b. n'i $[q a j \dot{i}-m]_{P . A C C}$ ašša qat-ti- $\eta \dot{i}-t \dot{t}$.

NEG what-ACC NEG kill-IPVF-AOR-3SG.O

'Er tötet nichts.'

(AAI_1973_Okyle_flk_8.2, N: Taz)

c. Na čēli [lōs-ira] $]_{S}$ n’i kuččä ašša qən-na.

this day devil-old.man NEG where NEG go.away-AOR.3SG

'An diesem Tag ging der teuflische Alte nirgendwohin.'

(AVA_1973_Ichakicha2_flk_77, N: Taz)

(200) a. $[K u d=n a j]_{A} \overline{\boldsymbol{a}} \quad q o-\check{s}-t \dot{t}-d \quad[t a \bar{b}-\mathcal{\varepsilon}-p]_{P . A C C}$ who $=$ EMPH NEG sight-US-IPFV-3SG.O 3SG-EP-ACC

'Niemand erkannte sie.'

(TTD_1964_Frog_flk_59, Z: Tym)

b. tab-ne $\quad[k \bar{u} d=n e j]_{A}$ az üngo-lš-ku- $\eta$.

3SG-DAT who = EMPH NEG listen-INT.PF-ITER-3SG.S

'Niemand hört auf sie.'

(PMP_1966_BodylessHead_flk_94, S: Middle Ob)

c. $[Q a j-m=n a j]_{P . A C C} \overline{\boldsymbol{a}}$ kadi-d tab-ə-nd.

what-ACC $=$ EMPH NEG tell-3SG.O 3SG-EP-ILL

'Sie hat ihm nichts erzählt.'

(SAA_1971_ThreeSisters_flk_59, Z: Narym)

d. $[\text { Qənnay }]_{A}[q a i-m=n]_{P . A C C}$ as qwet-na-t.

dog what-ACC $=$ EMPH NEG catch-AOR-3SG.O

'Der Hund hat nichts gefangen.'

(KKN_1971_FiveCarpBrothers_flk_55, S: Upper Ket')

e. Kudi-tko=naj $\overline{\boldsymbol{a}}$ jari-mba.

who-TRL $=$ EMPH NEG be.afraid-PST.REP.3SG

'Er hatte vor niemandem Angst.'

(MNS_1984_DaughterOfEarth_flk_2, Z: Narym)

f. $[\mathrm{Tab}]_{\mathrm{A}}$ kun $=n a j$ ad'uka-nde-nnan aya

3SG where $=\mathrm{EMPH}$ grandmother-OBL.3SG-EL2 NEG

kwin-ba.

go.away-PST.REP.3SG

'Er ging nirgendwohin weg von der Großmutter.'

(ChDN_1983_ItjaStayedAlone_flk_5, Z: Vasjugan) 


$$
\begin{aligned}
& \text { g. ... kuza-n=nej as qo-nze-r-sa-u [ta-nani } \\
& \text { when-LOC.ADV = EMPH NEG sight-IPFV-FRQ-PST-1SG.O 2SG-ADE } \\
& \text { nan'a-m- } t]_{P . A C C} \text {. } \\
& \text { sister-ACC-3SG } \\
& \text { 'Ich habe deine Schwester niemals gesehen.' } \\
& \text { (PMP_1966_ForestWoman }{ }_{f} \text { lk_215, S: Middle Ob) }
\end{aligned}
$$

Anders als in den zentral- und südselkupischen Dialekten kann im Nordselkupischen in negierten Präteritalsätzen auch das negative Existenzialverb č $’ a ̈ g k \dot{t}$ als Negationsmarker in der 3. Person Singular fungieren. Es trägt keine Tempusmarkierung und erscheint satzfinal, wie in Existenzialsätzen (siehe auch Abschnitt 4.4.2 unten). Dem Negationsmarker geht das lexikalische Verb in einer nominalisierten Form voraus (Nomen actionis auf -ptä). Darüber hinaus gibt es keine weitere Präteritalmarkierung, das üblicherweise verwendete Präteritalsuffix $-s$ erscheint nicht. Das folgende Beispiel stammt aus Wagner-Nagy (2011), da im Textkorpus diese Variante nicht belegt ist.

(201) man ili-ptä-mi் č'ä̈mka.
1sG live-NMLZ-1sG NEG.EX.3sG
'Ich lebte nicht.'
(Wagner-Nagy (2011: 109), N: Taz)

Belegt sind dagegen Beispiele, in denen č $\breve{a ̈ j k i ~ v o r ~ d e m ~ l e x i k a l i s c h e n ~ V e r b ~ a l s ~ N e g a-~}$ tionspartikel erscheint, das lexikalische Verb jedoch noch nominalisiert ist, wie in (202).

(202) ... ämtä qaj-ì-m čä̈̄jki me-ptä-ti் mačín lossi.

even what-EP-ACC NEG do-NMLZ-3SG taiga-GEN devil

'... sogar der Waldteufel machte nichts.'

(SNS_1966_Markincha_flk_173, N: Taz)

\subsubsection{Negierte Kopulasätze}

In Anlehnung an Dixon (2010b: 159) enthalten affirmative Kopulasätze eine Kopula als Prädikat sowie zwei Komplemente - Kopula-Subjekt und Kopula-Objekt (Kapitel 4.1.3). Das Selkupische verfügt zwar nicht über ein affirmatives Existenzialverb,

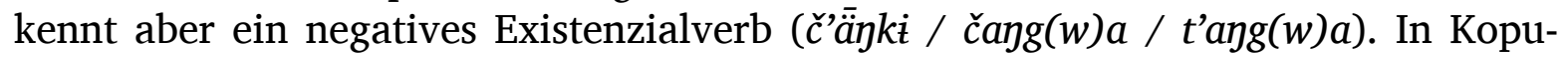
lasätzen werden die Negationspartikeln a ̌̌ša und das negative Existenzialverb zur Negation verwendet. Ausschließlich in zentralselkupischen Dialekten ist die Verwendung des aus dem Russischen stammenden Negationswortes n'etu belegt, das dort als negatives Existenzialverb interpretiert wird.

Im südselkupischen Beispiel (203) wird mit einer Kopulakonstruktion eine Eigenschaft (parsay - 'runzlig') des Kopulasubjektes ( $n$ 'epsol - 'deine Brust') negiert. Die Negation erfolgt an der Kopula $\bar{e}$, die lexikalisch overt ausgedrückt ist und mit der Variante as der Negationspartikel negiert wird. 
(203) N'epso-l nano $[\text { parsa- } \eta]_{A D V P}$ as $[j e-\eta]_{C O P}$. breast-2SG then wrinkly-ADV NEG be-3SG.S

'Deine Brust ist wohl nicht runzlig.' (PMP_1961_ForestWoman_flk_156, S: Middle Ob)

Die wichtigste Funktion des negativen Existenzialverbs ist das Ausdrücken von Negation in Existenzialsätzen. In Kapitel 4.1.3 ist bereits beschrieben worden, dass sich Existenzial-, Possessiv- und Lokativsätze im Selkupischen lediglich durch die Wortfolge des Themas und der Lokation voneinander unterscheiden lassen. Thema und Lokation erscheinen auch in negierten Existenzialsätzen in der Reihenfolge LOCATION + THEMA + KOPULA (vgl. auch Wagner-Nagy (2011: 208)). Die finite Verbform erscheint satzfinal. Das negative Existenzialverb hat im Nordselkupischen die Form $\check{c}$ 'ä̈lki, in zentralen Dialekten čang(w)a und t'ang(w)a, in den südlichen Dialekten wird nur die Form $t^{\prime} \ddot{a} \eta g(w) q$ verwendet. Das Thema kann mit einem Possessivsuffix markiert sein, wie in (204a), es kann aber auch unmarkiert vorkommen, wie in (204b) (204d). In Beispiel (204e) ist das Thema durch ein Nomen im Translativ ausgedrückt, in (204f) ist es ein Interrogativpronomen, an das die emphatische Partikel naj angehängt ist, wie auch in den Beispielen (200a) - (200g) in Abschnitt 4.4.1. Ein lokatives Element ist teilweise vorhanden, in (204b) sowie (204d) - (204f). In den Beispielen (204a) und (204c) hingegen gibt es kein overtes lokatives Element.

$$
\begin{aligned}
& \text { a. ... [ämä-mì }]_{T H} m \bar{e} l \quad[\check{c} \ddot{a} \bar{\eta} k a]_{C O P} \text {. } \\
& \text { mother-1SG always NEG.EX.3SG }
\end{aligned}
$$

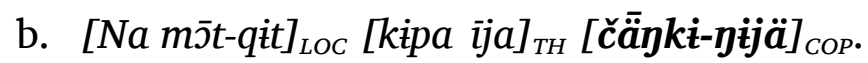

this tent-LOC small child NEG.EX-IMP.3SG.S

'In diesem Zelt soll kein Kind sein!'

(NN_1973_Shaman_flk_12, N: Taz)

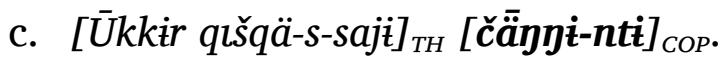

one ant-GEN-eye NEG.EX-INFER.3SG

'Es gibt nicht einen Stern.'

(AVA_1973_Ichakicha2_flk_111, N: Taz)

d. [Mì-nan $]_{L O C}$ nìn $[q o z a r]_{T H}[t \text { ä̈ggwa }]_{C O P}$.

1PL-ADE here mammoth NEG.EX.3SG

'Bei uns gibt es keine Mammuts.'

(KFN_1967_Mammoth_flk_1, Z: Tym)

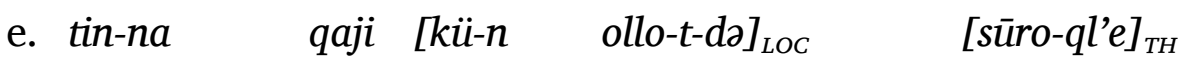

here-ABL.AN what river-GEN head-LOC.ADV-3SG hunt-TRL

$\left[\boldsymbol{t}^{\prime} \ddot{a} \boldsymbol{\eta} \mathbf{u}\right]_{\mathrm{COP} \text {. }}$

NEG.EX.3SG

'Von nun an gibt es keine Jagd am Oberlauf des Flusses.'

(KNI_1964_ItjaCapeOfWorms_flk_64, S: Upper Ket') 
f. [Kuačo-gat $]_{L O C}[\mathrm{kai}=n e]_{T H} \quad[\text { čaju-a-n }]_{C O P}$. city-LOC what $=$ EMPH NEG.EX-AOR-3SG.S

'In der Stadt war keiner.'

(NN_1855_Hero3_song_221, Z/S: Tym/Middle Ob)

Im zentralselkupischen Beispiel (205) wird mit der aus dem Russischen stammenden Negationspartikel netu das negative Existenzialverb čāngwa - 'er ist nicht da' negiert. Da es das einzige Beispiel dieser Art im Korpus ist, lässt sich nicht mit Gewissheit sagen, ob das negative Existenzialverb tatsächlich negiert werden kann].

(205) $\left[\text { T'emn'a }_{T H} \text { n'etu [čāngwa }\right]_{C O P}$, [hör $]_{T H}[\check{c} \bar{a} \eta g w a]_{C O P}$.

brother NEG NEG.EX.3SG snow NEG.EX.3SG

'Weder Bruder noch Schnee sind da.'

(MNS_1984_BrotherSister_flk_17, Z: Narym)

Des Weiteren wird nur im Zentralselkupischen die Negationspartikel netu als negatives Existenzialverb reinterpretiert und zur Bildung negierter Existenzialsätze (Beispiele (206a) und (206b)) und Lokativsätze (Beispiel (206c)) verwendet. Im Korpus sind die Varianten netu und n'etu belegt. Das negative Existenzialverb kann sowohl Tempusmarkierung tragen, wie in (206b), als auch Personenmarkierung wie in (206c). In (206a) sind die beiden negierten Existenzialsätze durch Juxtaposition korrelativ im Sinne von 'weder ... noch' miteinander verbunden.

(206) a. [T'emn'a-dit $]_{T H}\left[\boldsymbol{n}^{\prime} e t u\right]_{C O P}, \quad[t o l ' \zeta ̌ e-n \text { wat-tit }]_{T H}\left[\right.$ n'etu $_{C O P}$. brother-3SG NEG.EX.3SG ski-GEN road-3SG NEG.EX.3SG

'Es gibt weder einen Bruder noch eine Skispur.'

(MNS_1984_BrotherSister_flk_23, Z: Narym)

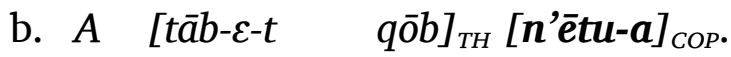

but 3SG-EP-GEN skin NEG.EX-AOR.3SG

'Aber ihre Haut war nicht da.'

(TTD_1964_Frog_flk_75, Z: Tym)

c. $[\text { Kuča-d naj }]_{T H}[\text { netu- } k]_{C O P}$.

where-ADV EMPH NEG.EX-3SG.S

'Er ist nirgendwo.'

(MNS_NN_BullSon_flk_184, Z: Narym)

Das negative Existenzialverb wird auch verwendet, um Negation in Lokativsätzen auszudrücken. Wie bereits oben angeführt, lässt sich ein Lokativsatz lediglich anhand der Abfolge von Thema und Lokation vom Existenzialsatz unterscheiden. Das Prädikat erscheint in der Regel, wie im Existenzialsatz, satzfinal. Die Wortfolge im Lokativsatz ist THEMA + LOKATION + KOPULA. Anders als in Existenzialsätzen ist die Angabe eines lokativen Elements in Lokativsätzen obligatorisch (siehe auch die Ausführungen zu affirmativen Kopulasätzen in 4.1.3). Die Beispiele (207b) und (207c) weisen

\footnotetext{
${ }^{6}$ Wagner-Nagy (2011: 210) nimmt an, dass dies - zumindest mit der Negationspartikel ašša - nicht möglich ist.
} 
die für Lokativsätze typische Wortfolge auf, im nordselkupischen Beispiel (207a) erscheint das Verb abweichend satzinitial. Die Themata sind in allen Beispielen ohne Possessivmarkierung.

a. $[\check{C} \bar{a} \eta k a]_{C O P}[\text { nimtit }]_{L O C}[\ddot{i j a}]_{T H}, \ldots$ NEG.EX.3SG here child

'Nicht hier ist der Junge, ...' (MIV_1977_Icha_flk_33, N: Upper Taz)

b. $[\text { Tap }]_{T H}[\text { màt-qan }]_{L O C}\left[t^{\prime} \ddot{a ̈ j w a-n}\right]_{C O P}$ 3SG house-LOC NEG.EX-3SG.S

'Er ist nicht im Haus.'

(PMP_1961_Fairytale_flk_146, S: Middle Ob)

c. $[Q \partial n a \eta-n \partial]_{T H}[\mathrm{mät}-q a n]_{L O C}[\mathbf{t} \text { 'angu }]_{C O P}$. dog-1SG house-LOC NEG.EX.3SG

'Mein Hund ist nicht im Haus.'

(KKN_1971_FiveCarpBrothers_flk_51, S: Middle Ket')

Possessivsätze verfügen über dieselben zugrundeliegenden Strukturen wie Existenzial- und Lokativsätze. Der Possessor entspricht dem lokativen Element und das Possessum dem Thema. Possessive Konstruktionen weisen die gleiche Wortfolge wie Existenzialsätze auf: Possessor (PoS) + Possessum (PoM) + Kopula (COP). Wie in affirmativen Sätzen liegt der (formale) Unterschied zwischen beiden Relationen sowohl in der obligatorischen Angabe des Lokativ-Komplements in Possessivsätzen, als auch in dessen Animatheit: Das Lokativ-Komplement in Existenzialsätzen ist inanimat, wie z. B. kuačogat - 'in der Stadt' in Beispiel (204f). In Possessivsätzen ist das Lokativ-Komplement hingegen animat, wie in den folgenden Beispielen (208a) (209e). In negierten Possessivsätzen wird unabhängig von der Possessormarkierung das negative Existenzialverb č $\breve{a} j k \dot{z}$ (und seine Varianten) verwendet. Es erscheint typischerweise satzfinal. In allen drei Dialektgruppen kann der Possessor mit einer unmarkierten Nominativ-NP ausgedrückt werden, wie in den Beispielen (208a), (208b) und (209f). Zusätzlich ist eine Lokativ-Markierung des Possessors möglich (Location Schema bei Heine (2009: 50), Locational Possessive bei Stassen (2001: 955)), wobei sich die nördlichen Dialekte von den zentralen und südlichen unterscheiden. Im Nordselkupischen wird, wie in (208c), eine Adpositionalphrase verwendet, die aus dem postpositionalen Kopf miqin - 'bei' und dem nominalen Genitiv-Modifikator iman 'Frau.GEN' besteht. Negierte Konstruktionen mit einer Genitivmarkierung (Genitive Schema bei Heine (2009: 58), Genitive Possessive bei Stassen (2001:956)) des Possessors (vgl. die affirmativen Beispiele (153a) und (153b) in Kapitel 4) sind im Korpus nicht belegt.

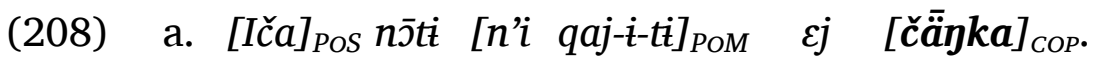
Itja then NEG what-EP-3SG and NEG.EX.3SG

'Itja hatte dann nichts mehr.'

(AVA_1973_Ichakicha2_flk_45, N: Taz) 


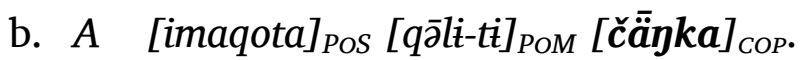
but old.woman fish-3SG NEG.EX.3SG 'Aber die Alte hat keinen Fisch.' (BEP_1973_Itja2_flk_3, N: Taz)

c. [Ima-n miqin $]_{P O S}[i r a-t i t]_{P O M}[\check{c} \bar{a} \bar{g} y-m \dot{\mathbf{i}}-\mathbf{m p a}]_{C O P}$. woman-GEN near husband-3SG NEG.EX-DRV-PST.REP.3SG

'Die Frau hatte keinen Mann.' (MIV_1977_Icha_flk_3, N: Upper Taz)

In den zentralen und südlichen Dialekten wird das Lokativkomplement morphologisch mit dem Adessiv-Suffix -nan ausgedrückt. Dabei können sowohl Interrogativpronomina wie in (209a), als auch Personalpronomina wie in (209b) - (209d) markiert werden. In Beispiel (209e) ist der Possessor nicht lexikalisch overt ausgedrückt, er wurde zuvor im Kontext benannt. Das Possessum ütč' elat - 'Kinder' erscheint satzinitial und wird damit betont. Das Prädikat in Form des negativen Existenzialverbs steht typischerweise satzfinal.

a. Tat čad $[\text { kudï-nnan }=n a j]_{P O S}\left[t^{\prime} \text { ana }\right]_{C O P} \quad[t \bar{u}]_{P O M}$. 2SG because. of who-LOC.AN = EMPH NEG.EX.3SG fire

'Deinetwegen hat niemand Feuer.'

(ChDN_1983_MistressOfFire_flk_72, Z: Vasjugan)

b. $[M a-n a n]_{P O S}[k o m d \varepsilon]_{P O M}[\check{c} a j w a]_{C O P}$.

1SG-ADE money NEG.EX.3SG

'Ich habe kein Geld.'

(MNS_NN_BullSon_flk_11, Z: Narym)

c. [Tab-la-nan $]_{P O S}\left[\varepsilon\right.$ l'mād-la $_{\text {POM }}\left[\boldsymbol{t}^{\prime} \mathbf{a} \boldsymbol{\eta} \text { u-za-t }\right]_{C O P}$.

3SG-PL-ADE child-PL NEG.EX-PST-3PL

'Sie hatten keine Kinder.'

(SEV_1980_HazelGrouse_flk_2, S: Middle Ob)

d. ... a [me-nan $]_{P O S}\left[t^{\prime} a j u\right]_{C O P}\left[\check{c}^{\prime} u p a-l a-w\right]_{P O M}$. but 1SG-ADE NEG wing-PL-1SG

'... aber ich habe keine Flügel.'

(KKA_NN_HazelGrouse_flk_8, S: Upper Ket')

e. [Ütče-la-t $]_{P O M}\left[\boldsymbol{t}^{\prime} \mathbf{a} \boldsymbol{g} \boldsymbol{u}-\mathbf{k u}-\boldsymbol{s}\right]_{\text {COP. }}$.

child-PL-3SG NEG.EX-ITER-PST.3SG

'Kinder hatte sie nicht.'

(KKN_1971_WhiteHeadedWoman_flk_2, S: Middle Ket')

f. Nuzel'i qallo $\left[\mathrm{man}_{P O S}[0 n n a ̈-n \quad k u w-a-j \quad \text { pänžarmì }]_{P O M}\right.$ really supposedly $1 \mathrm{SG}$ himself-GEN person-EP-ADJZ environment

$\left[t^{\prime} \ddot{y} y u\right]_{C O P}$ ?

NEG.EX.3SG

'Habe ich wirklich keine menschliche Umgebung?'

(SVG_1964_ItjeFatherFishMotherFish_flk_4, S: Upper Ket') 


\subsubsection{Negierte verblose Sätze}

Auch in negierten Kopulasätzen kann die Kopula $\bar{e}$ lexikalisch kovert sein, wie in den Beispielsätzen (210a) - (210c). In den ersten beiden Beispielen werden dem grammatischen Subjekt Eigenschaften zugeschrieben bzw. wird deren Fehlen ausgedrückt. Die Negationspartikeln ała bzw. assə gehen dem Kopulakomplement voraus. Beispiel (210c) zeigt einen negierten Existenzialsatz, die Negationspartikel as erscheint regulär satzfinal.

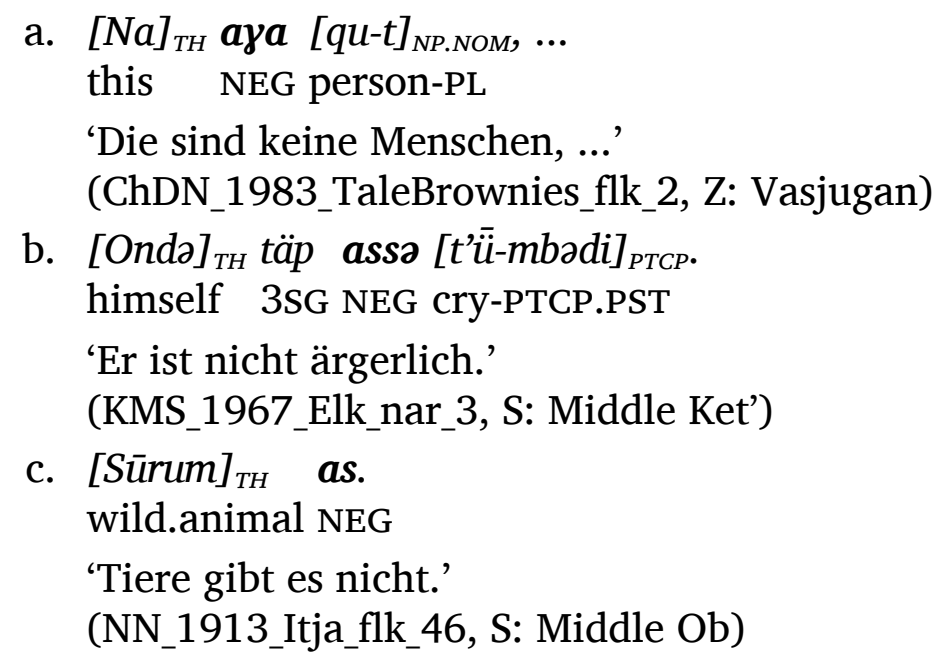

\subsubsection{Negierter Imperativsatz}

Negierte Imperativsätze werden im Selkupischen nicht mit der Standardnegationspartikel ašša gebildet, sondern mit der imperativischen Negationspartikel iki (Nordselkupisch), igi, ög (Zentralselkupisch) und $i k(\dot{i}), \partial g(\partial)$ (Südselkupisch). Ebenso wie $a \check{s} \check{s} a$ erscheint $i k \dot{z}$ unmittelbar vor dem Prädikat. Im Textkorpus gehen alle Argumente des Verbs typischerweise dem negierten Prädikat voraus, wie in (211a), (211c) und (211e). Die umgekehrte Reihenfolge ist jedoch ebenso belegt, wie in (211b), (211d) und (211f). Nur in nördlichen Dialekten finden sich Belege, in denen die Kurzform eines (personal)pronominalen Objektes zwischen der Negationspartikel und der finiten Verbform erscheint wie in Beispiel (211g). Einzig das Subjekt, sofern es lexikalisch overt ausgedrückt wird, erscheint stets satzinitial, wie in (211a) - (211c). In den Beispielen (211d) - (211f) ist das Subjekt covert, die Markierung erfolgt an der finiten Verbform, in (211f) ist auch das direkte Objekt covert, es wurde zuvor im Kontext erwähnt.

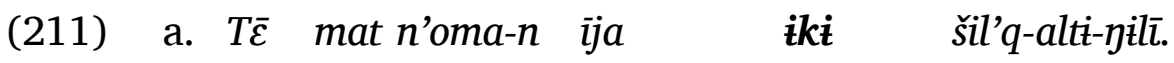

2DU 1SG hare-GEN child.NOM NEG.IMP spoil-DRV-IMP.2DU

'Ihr verderbt mein Häschen nicht!'

(AVA_1973_Ichakicha2_flk_79, N: Taz)

b. Tan iki $\bar{u} t \ddot{i}-t \dot{t}$ na qup.

2SG NEG.IMP let.go-IMP.2SG.O this person.NOM 
'Du lass diesen Menschen nicht gehen!'

(BEP_1973_Fat2_flk_17, N: Taz)

c. Tan man nodik igi twi-l'-ed!

2SG 1SG sterlet.NOM NEG.IMP steal-INCH-2SG.O

'Du stiehl meinen Stör nicht!'

(ChDN_1983_Nikita_flk_27, Z: Vasjugan)

d. izy qwad-ěšik mašik!

NEG.IMP kill-IMP.SG.S 1SG.ACC

'Töte mich nicht!'

(TTD_1964_Frog_flk_97, Z: Tym)

e. Täb-i-ni uto-yinda ikk a mi-ku-k!

3SG-EP-DAT hand-ILL.3SG NEG.IMP give-ITER-IMP.2SG.S

'Fall ihm nicht in die Hände!'

(KMS_1967_Bear_flk_2, S: Middle Ket')

f. Tidam ik mer-i-m-p-et nā

now NEG.IMP price-EP-TRL-HAB-IMP.2SG.O this

nä-j-qum-ne!

woman-ADJZ-person-DAT

'Jetzt verkauf (ihn) nicht dieser Frau!'

(PMP_1961_Fairytale_flk_57, S: Middle Ob)

g. Tōmnänka lajkì-n'-n'a: Tō iki $\quad$ šlp čatt-äšik!

Tomnenka cry-VBLZ-AOR.3SG away NEG.IMP 1sG.ACC throw-IMP.2sG.S

'Tomnenka schrie: Wirf mich nicht weg!'

(KaIA_1973_Natenka_flk_36, N: Taz)

Für das Nordselkupische beschreibt Wagner-Nagy (2011: 144ff.) die Verwendung der negativen Imperativpartikel $i k i$ als einzige Möglichkeit zur Negation auch in Optativkonstruktionen wie in (212).

$$
\begin{aligned}
& \text { Tat təp-i-m iki čati-lä-l } \\
& \text { 2SG 3SG-EP-ACC NEG.IMP meet-OPT-2sG.O } \\
& \text { 'Triff ihn/sie nicht mehr!' } \\
& \text { (Wagner-Nagy (2011: 146), N: Taz) }
\end{aligned}
$$

Der Optativ wird mit dem Suffix -lä円 markiert und impliziert eine futurische Referenz. Im Textkorpus finden sich nur in südselkupischen Texten Belege, in denen negierte Optativsätze verwendet werden, wie in (213a) und (213b). Im nordselkupischen Subkorpus sind negierte Optativsätze nicht vorhanden, im zentralsekupischen Subkorpus wird in negierten Optativsätzen überwiegend die Standard-Negationspartikel $a \check{s ̌} a$

\footnotetext{
${ }^{7}$ Das Suffix -lä bezeichnet in Norddialekten den Optativ, in Nicht-Norddialekten hingegen ist das Suffix ein Futurmarker (-ntV in Norddialekten). Der Grund für die Unterschiede liegt in der unterschiedlichen Entwicklung der Futurmarker in Nord- und Nicht-Norddialekten (vgl. auch Cheremisina/Martynova (1991: 17) und Wagner-Nagy (2015: 5))
} 
verwendet, wie die Varianten $\bar{a}$ und aya in (214a) und (214b). Beispiele mit der imperativen Negationspartikel sind lediglich vereinzelt belegt, wie in (214c). Aufgrund der geringen Anzahl von Beispielen lässt sich bisher nicht sagen, ob die Standardnegationspartikel tatsächlich auch in optativen Konstruktionen verwendet werden kann oder ob die unterschiedliche Verwendung beispielsweise sprecherabhängig ist.

a. Kib-anno-nd og omdi-k-le-nd! small-boat-ILL NEG.IMP sit.down-ITER-OPT-2SG.O

'Du darfst dich nicht in das Boot setzen!' (SEV_1981_SisterBrother_flk_19, S: Middle Ob)

b. iki tï̈-r-a-le-nde! NEG.IMP cry-FRQ-EP-OPT-2sG.S

'Du sollst nicht weinen!' (TET_1979_SnowGirl_flk_13, S: Middle Ket')

a. Čèl-n nē mašik $\bar{a}$ üdì-lla. sun-GEN daughter 1SG.ACC NEG let.go-OPT.3SG

'Die Tochter der Sonne will mich nicht gehen lassen.' (MNS_1984_DaughterOfEarth_flk_28, Z: Narym)

b. T'ekka pō-m aya m'e-l'e-b'e. 2SG.DAT tree-ACC NEG give-OPT-1SG.O 'Ich möchte dir kein Holz geben.' (ChDN_1983_MistressOfFire_flk_21, Z: Vasjugan)

c. Tat $\dot{\text { iy lari-mb-le-nd } \quad k u d i-m=n a j a . ~}$ 2SG NEG.IMP be.afraid-HAB-OPT-2sG.S who-ACC = EMPH

'Du brauchst dich vor niemandem fürchten.' (TTD_1964_CalfHare_flk_23, Z: Tym)

\subsubsection{Negierter Interrogativsatz}

Negierte Interrogativsätze werden auf die gleiche Art und Weise gebildet wie negierte Deklarativsätze (Abschnitt 4.4.1), indem die Negationspartikel ašša der finiten Verbform vorangestellt wird. Bei den sogenannten w-Interrogativsätzen, die mit einem Interrogativpronomen eingeleitet werden, erscheint dieses typischerweise satzinitial, wie in den Beispielen (215b) und (215c). Eine andere Wortfolge ist jedoch ebenfalls möglich, wie in (215a). Polare Interrogativsätze wie in (216a) und (216b) unterscheiden sich formal nicht von ihrem affirmativen Pendant (siehe Kapitel 4.2). Da negierte Interrogativsätze im Korpus nur selten vorkommen, fehlt das Material, um detailliertere Analysen vornehmen zu können.
a. Tat qum-i-t-i-p
qajï-tqo ašša aps-tí-sa-l?
2SG person-EP-PL-EP-ACC what-TRL NEG eat-TR-PST-2SG.O 
'Warum hast du den Menschen nichts zu essen gegeben (wrtl.: nicht gefüttert)?'

(BVP_1973_East_flk_22.3, N: Taz)

b. Kaj-ko mi $\overline{\boldsymbol{a}}$ kwen-ba-j?

what-TRL 1PL NEG go.away-PST.REP-1DU

'Warum sind wir nicht gegangen?'

(MNS_NN_BullSon_flk_85, Z: Narym)

c. Aža, kai-tko asa sut kit-e-ggu-sa-nd?

father what-TRL NEG 1PL.ACC say-EP-ITER-PST-2SG.S

'Vater, warum hast du es uns nicht gesagt?'

(NN_1855_Hero1_song_102-103, Z/S: Tym/Middle Ob)

(216)

a. Q-as tinno-wa-l'i ass kät-ku-wa-l'i?

-NEG know-AOR-2SG.O NEG Say-ITER-AOR-2SG.O

'Weisst du es nicht oder sagst du es nicht?'

(SVG_1964_ItjeFatherFishMotherFish_flk_28, S: Upper Ket')

b. Nä-l-gum asa ko-a-l?

woman-ADJZ-person NEG find-AOR-2SG.O

'Hast du kein Weib gefunden?'

(NN_1855_Hero1_song_26, Z/S: Tym/Middle Ob)

\section{Zusammenfassung}

Summierend lassen sich im Selkupischen die drei Satzmodi Deklarativsatz, Interrogativsatz und Imperativsatz unterscheiden. In allen Satztypen kongruiert die finite Verbform in Numerus und Person mit dem syntaktischen Subjekt, das durch eine (pro)nominale Phrase ausgedrückt wird. Die lexikalische Realisierung ist bei pronominalen NPs nicht obligatorisch, sie können „gedropped“ werden, wie beispielsweise in (124), (125c) oder (126d). In dem Fall wird mit Hilfe der Verbalendung auf das Subjekt referiert. In Deklarativ- und Imperativsätzen werden die Kategorien Person, Numerus, Tempus und/oder Modus morphologisch an der finiten Verbform markiert. Deklarativsätze beinhalten neben intransitiven auch mono- und ditransitive Sätze. Neben den typischen ditransitiven Konstruktionen mit physikalischen Transferverben (Beispiele (129a) - (137)) werden auch kognitive und mentale Transferverben wie in (139a) - (141c) sowie benefaktive Konstruktionen wie in (142) - (143b) verwendet. Direkte Objekte können im Akkusativ oder Nominativ realisiert werden. Beide Varianten kommen jeweils mit subjektiver und objektiver Konjugation vor.

Innerhalb von Kopulasätzen können im Selkupischen mit einer Vielzahl von Komplementen unterschiedliche Relationen ausgedrückt werden. Hierzu zählen Identität (Nominativ-NP, Pronomen), Attribution (Adjektiv, Partizip, Adverb, InstrumentalNP, Karitiv-NP), Possessivität (Genitiv-NP, Lokativ-NP), Lokation (Lokativ-NP, Lokativadverb), Existenz (Lokativ-NP) sowie Zustandsänderungen (Translativ-NP) (vgl. 
auch Tabelle 16). Als Kopula wird typischerweise $\bar{e}-$ 'sein' verwendet, je nach Komplement fungieren im Korpus auch andere Verben wie $\varepsilon s \dot{z}$ - 'werden' oder orim 'wachsen' als Kopula.

In Abschnitt 4.2 wurden eingeleitete (Beispiele $(179 a)-(186 c)$ ) und uneingeleitete (Beispiele (187a) - (187c)) Interrogativsätze beschrieben. Interrogativwörter erscheinen in der Regel satzinitial und verfügen im Textkorpus nur noch über unvollständige Kasus- und Numerusparadigmen.

Für Imperativsätze konnte festgestellt werden, dass das ursprünglich vollständige Konjugationsparadigma für die subjektive und die objektive Konjugation heute nur noch burchstückhaft erhalten ist. In Südselkupischen Texten lassen sich noch alle Personen und Numeri (Singular, Dual, Plural) belegen, im Zentralselkupischen nicht mehr. Formen der 2. Person kommen am häufigsten vor. Wie in Deklarativsätzen können auch in Imperativsätzen Nominativ- und Akkusativobjekte verwendet werden. Dabei sind ebenfalls beide Konjugationsarten mit beiden Objektmarkierungen belegt.

Sämtliche affirmativen Satzmodi sind auch negiert belegt. Hierfür werden unterschiedliche Negationspartikeln ( $a \check{s ̌ s} a$ bzw. eine Variante in Deklarativsätzen, ǎšs und $n ' e$ bzw. eine Variante in Interrogativsätzen, iki in Imperativsätzen) der finiten Verbform vorangestellt. In den Heldenliedern aus der gemischten Dialektgruppe kann zwischen der Negationspartikel und der finiten Verbform ein pronominales direktes oder indirektes Objekt erscheinen wie in Beispiel (215c). 


\section{Komplexer Satz}

Zwei oder mehr Teilsätze, die durch Koordination oder Subordination miteinander verbunden werden, ergeben traditionell einen komplexen Satz. Aus einer indoeuropäischen Sichtweise heraus wird ein Teilsatz („clause“) traditionell durch das Vorhandensein einer finiten Verbform und eines expliziten Subjekts definiert. Infinite Konstruktionen formten demnach keine formal komplexen Sätze, da ihnen die Merkmale Finitheit und explizites Subjekt fehlen. Da das Selkupische ursprünglich, wie die anderen uralischen Sprachen auch, über keine genuinen Satzkonjunktionen verfügt, werden komplexe Sachverhalte originär mittels Juxtaposition und infiniten Verbformen (Nominalisierungen, Partizipien, Konverben) ausgedrückt (Helimski 1998: 576 f.).

Gerade in Konstruktionen mit infiniten Verbformen, vor allem Konverben, ist eine genaue Abgrenzung zwischen koordinierten und subordinierten Sachverhalten häufig nicht eindeutig möglich, da oft mehrere Lesarten denkbar sind und die russische Übersetzung als Anhaltspunkt nur ungenügend ist. Hier bieten parametrische Ansätze (Haiman/Thompson 1984; Lehmann 1988; Bickel 1991, Bickel 1993, Bickel 2010) im Gegenteil zu traditionellen Ansätzen die Möglichkeit, Konstruktionen zu betrachten, die sich der traditionellen Dichotomie Koordination/Subordination nicht zuordnen lassen (vgl. Folev/Van Valin Jr. (1984): Van Valin Jr. (2005); Thompson/ Longacre/Hwang (2007); Longacre (2007); Comrie (2008)). Satzverknüpfungen werden dort als ein Kontinuum betrachtet werden, zwischen deren typologisch relevanten Parametern (vgl. Lehmann 1988: 3) Satzverknüpfungsstrategien zugeordnet werden können.

Inwieweit der Begriff der Ko-Subordination (vgl. Foley/Van Valin Jr. (1984); Van Valin Jr. 2005) passender wäre oder ob eine Unterteilung in Koordination und Subordination zumindest für die infiniten Verbformen überhaupt passend ist, sind Fragen, mit denen sich die künftige Forschung hoffentlich auseinandersetzt. Zum gegenwärtigen Zeitpunkt fehlen zur Beantwortung der Fragen Forschungsergebnisse, die im Rahmen der vorliegenden Arbeit auch nicht erbracht werden können. So werden infinite Konstruktionen jeweils in koordinierende subordinierende Strukturen eingeordnet, auch wenn weitere Lesarten denkbar sind. Da Konverben im Selkupischen infinite Verbformen sind, die explizite Subjekte aufweisen können, wie in Beispiel (217), wird in Übereinstimmung mit Dixon (2010a: 109 f.) und Haspelmath (1995: 11) der traditionelle Teilsatz-Begriff ("clause“) modifiziert und muss obligatorisch mindestens ein Prädikat enthalten. Damit formen Konverben und andere infinite Formen konverbale bzw. infinite Teilsätze. Verfügen Teilsätze über identische Prädikate, kann ein Teilsatz zudem elliptisch sein, wie in den Beispielen (232a) und (232b) unten. 
(217) Tepqin ${ }_{S 1} q w e l e-w l ' e_{P R E D 1}, I t ' e_{S 2}$ amdi-qu- $\eta_{P R E D 2}$.

3DU fish-CVB Itja sit-ITER-3SG.S

'Sie gingen fischen und Itja sitzt.'

(KKN_1971_Itja_flk_40, S: Upper Ket')

Neben der Juxtaposition existieren aus dem Russischen entlehnte koordinierende und subordinierende Konjunktionen, die ebenfalls komplexe Sätze bilden. Innerhalb eines komplexen Satzes können unmarkjerte Objekte und Akkusativobjekte gemeinsam vorkommen, wie in (218a) und (218b).

a. Ära [kol'd-i-t kwil] $]_{N P . N O M} k w a t-p a-t, \quad$ tab [kol'd-i-t old.man Ob-EP-GEN fish.NOM kill-PST.REP-3SG.O 3SG Ob-EP-GEN

$k w i l i-p]_{N P . A C C}$ olo-unde ketti-mba-t, ando-yonde

fish-ACC head-PROL beat-PST.REP.3SG.o boat-LOC.3SG

pen-ba-t.

put-PST.REP-3SG.O

'Der alte Mann fing einen Weißlachs, er schlug auf den Fischkopf, legte ihn ins Boot.'

(ChDN_1983_Nikita_flk_18, Z: Vasjugan)

b. Paja-nt-ne t'ari- $\eta$ tan $[s a c ̌]_{N P . N O M}$

woman-OBL.3SG-DAT/ALL say-3SG.S 2sG sliver.NOM

pör-č́t-q, [sega-m] $]_{N P . A C C}$ pör-ču-gu, k'er'eg'ey

round-VBLZ-IMP.2sG.S ball-ACC round-VBLZ-INF be.necessary

poni- $\eta$ sor-mi-gu.

net-ACC bind-COND-INF

'Er sagte zu seiner Frau: Spinne ein Band um ein Knäuel zu wickeln, man brauchst es, um ein Netz zu knüpfen!'

(PMP_1966_BodylessHead_flk_3, S: Middle Ob)

\subsection{Koordination}

Teilsätze mit gleichwertigem syntaktischen Status werden bei der Koordination (auch Parataxe) miteinander verbunden. Dabei spielen die verknüpften Ausdrücke in einem gegebenen Kontext die gleiche syntaktische Funktion. Von der syndetischen Koordination, die mittels Konjunktionen ausgedrückt wird, kann die asyndetische Koordination unterschieden werden, bei der die Teilsätze nebeneinander gestellt werden (Juxtaposition).

\subsubsection{Juxtaposition}

Die Satzverbindung mittels Juxtaposition (asyndetische Koordinaton) ist ein häufig verwendetes Mittel im Korpus. In den Beispielen (219a) und (219a) sind jeweils zwei 
Satzteile durch reine Nebeneinanderstellung verbunden. Die Beispiele formen additive Sachverhalte. Aufgrund einer fehlenden selkupischen Orthographie kann die Juxtaposition in den Texten ohne Satzzeichen wiedergegeben werden, wie in (219a), oder auch mit Komma, wie in (219b).
a. [Tü̈-t haj pakti-mba]
[üčed'e-l'i-ka-t
kilo-nd
fire-GEN eye jump-PST.REP.3sG child-DIM-DIM-GEN breast-ILL al't'i-mba].
fall-PST.REP.3SG

'Ein Funke (wrtl.: ein Auge des Feuers) flog auf und fiel auf die Brust des Kindes.'

(ChDN_1983_MistressOfFire_flk_14, Z: Vasjugan)

b. [Qwan-na], [poki-m i-qi-t], [nāgur t'āpi poqi-m-t]. go.away-AOR.3SG net-ACC take-ITER-3SG.O three float net-ACC-3SG

'Er ging los, nahm das Netz, das Netz mit drei Schwimmern.'

(TFF_1967_ItjaAldigaNet_flk_3, S: Middle Ob)

\subsubsection{Infinite Verbformen}

Wie bereits zu Beginn des Kapitels eingeführt, sind infinite Verbformen neben der Juxtaposition das ursprüngliche grammatische Mittel, um Satzverbindungen zu markieren. Konverben verfügen im Selkupischen über eine Vielzahl von Funktionen, von denen die „klassische“, die Bildung von Adverbialsätzen nur eine ist. Der gegenwärtige Stand der Forschung lässt keine eindeutige Zuordnung selkupischer Konverben zu. Sie werden in koordinierenden Satzverbindungen (Abschnitt (5.1.2)) ebenso verwendet wie in subordinierenden Satzgefügen (der folgende Abschnitt 5.2.2.1) und komplexen Prädikaten (Kapitel 6). Haspelmaths Terminus „cosubordination“ (Haspelmath 1995: 23 ff.) beschreibt den Status selkupischer Konverben teilweise, lässt jedoch die Möglichkeit von Konverben als Teil komplexer Prädikate außer acht.

\section{Koordinierende Konverbsätze}

Im Zentral- und Südselkupischen werden Konverben unter anderem verwendet, um eine Sequenz aufeinanderfolgender Satzteile (,clause-chaining“, vgl. Haspelmath (1995: 7), „medial-final chaining“ bei Longacre (2007: 375)) auszudrücken, insbesondere in den Ket'-Subdialekten des Südselkupischen. Hierbei erscheint die finite Verbform typischerweise im letzten Gliedsatz, die Teilsätze enthalten jeweils eine konverbale Form, die meistens final auftritt. Die Beispiele (220a) und (220b) zeigen jeweils einen komplexen Satz, der aus zwei Teilsätzen besteht, den konverbalen Formen können transitive Verben zugrunde liegen, wie katolešple - 'kratzen.CVB' in (220a), das über ein direktes Objekt olomd - 'Kopf.ACC' verfügt. Ein komplexer Satz kann mehrere konverbale Teilsätze enthalten, wie die Beispielsätze (220c) - (220e) zeigen, bis zu drei konverbale Teilsätze lassen sich im Textkorpus belegen, wie in 
(220e). Analoge zentralselkupische Beispiele gibt es im Korpus nicht. In den Teilsätzen können einfache und komplexe konverbale Formen kombiniert vorkommen, wie -le und lble in (220e).

Beispiel (220f) zeigt einen Satz, der als Kombination von koordinierenden und subordinierenden Konverben interpretiert werden kann. Hierbei sind der erste Teilsatz [Na čagirlebe] und der dritte Teilsatz [qoronక̌ $\varepsilon-n$ melladist] syntaktisch gleichwertig, der mittlere Teilsatz [swāq melebe] ist dem dritten Teilsatz als temporaler Nebensatz untergeordnet.

a. [Ara-l-žiga olo-m-d kato-le-šp-le], old.man-ADJZ-DIM head-ACC-3SG scratch-INCH-IPFV-CVB

[waze-mba].

get.up-PST.REP.3SG

'Der Alte kratzte sich den Kopf und stand auf.'

(MNS_NN_BullSon_flk_39, Z: Narym)

b. [Loz'i it'e-n moqqo-n üti-mb'i-l'e], [na mü-nda

devil Itja-GEN back-LOC.ADV let.go-HAB-CVB this hole-ILL

četčo-l'd'i- $\eta]$.

fall-INT.PF-3sG.S

'Loz lief hinter Itja und fiel in dieses Loch.'

(KKN_1971_Itja_flk_22, S: Upper Ket')

c. [Qanaך čenča-k:] [pelde-lebe], [mat loya-p tanga-lebe], [onž

dog say-3sG.O help-CVB 1SG fox-ACC throw.out-CVB himself qur-a-lla].

run-EP-OPT.3SG

'Der Hund sagt: Ich helfe dir, ich vertreibe den Fuchs, er soll von selbst weglaufen.'

(TET_1979_TheHaresHouse_flk_20, S: Middle Ket')

d. [Sēld'a siēnga-le], [ēr-le] [ām-dat].

seven overnight-CVB drink-CVB eat-3PL

'Sie übernachteten sieben Mal, tranken, aßen.'

(NN_1913_Itja_flk_116, S: Middle Ob)

e. [Lēr-le], [meša-lble], [pēqqì-n waže-m awēe-šp-le], [kundì

laugh-CVB dance-CVB elk-GEN meat-ACC eat-IPFV-CVB long

üd-i-m ü̈de-špa-dit].

water-EP-ACC drink-IPFV-3PL

'Sie lachten, tanzten, aßen Elchfleisch und tranken lange Wodka (wrtl.: Wasser).'

(MNS_1984_BrotherSister_flk_81, Z: Narym)

f. [Na čagi-r-lebe], [swāq me-lebe], [qoronžc-n me-lla-dist].

this dry-FRQ-CVB good do-CVB basket-ILL do-OPT-3PL

'So muss man ihn trocknen und, wenn er gut fertig ist, muss man ihn in 
einen Korb legen.'

(ILP_191981_HowSelkupLivedBefore_nar_4, S: Middle Ob)

\subsubsection{Konjunktionalsätze}

Konjunktionen als verbindende Elemente in koordinieren Sätzen sind im Selkupischen ursprünglich nicht vorhanden. Alle im Textkorpus belegten Konjunktionen sind aus dem Russischen entlehnt worden. Dazu zählen $i$ - 'und' ( $<$ russ. $i$ ), il'i - 'oder' ( $<$ russ. ili), $a$ - 'aber' (< russ. a) sowie aj - 'und' ( < russ. Partikel aj). Die russische Partikel $a j$ - 'und' wird im Südselkupischen als Konjunktion 'und' sowie als Adverb 'wieder' verwendet, im Zentralselkupischen erscheint sie ausschließlich als Adverb. Zusätzlich können die Temporaladverben nīni, kate, undo und nanto - 'dann' sowie die aus dem Russischen entlehnten to, potom und tagda als verbindendes Element fungieren.

\subsubsection{Additiv}

Die Konjunktion $i$ - 'und' wird in beiden Dialektgruppen vor allem additiv (X und Y) verwendet, wie in (221a) und (221a).
a. [[Aur-gu] [aj omde-špa-q]] i [qul-wat-pa-q eat-INF again sit.down-IPFV-3DU.S and talk-DRV-PST.REP-3DU.S ustol-t par-o-t]. table-GEN top-EP-LOC.ADV
'Sie setzten sich wieder, um zu essen und erzählten am Tisch.' (SAA_1971_ThreeSisters_flk_67, Z: Narym)
b. [Qü-la tö-mba-dit] i [awi-t aze-nd-se person-PL come-PST.REP-3PL and mother-3SG father-OBL.3SG-COM $m \bar{e} d i-m b a-q]$. achieve-PST.REP-3DU.S
'Die Leute kamen und die Mutter und der Vater kamen.' (TMR_1981_Robbers_flk_41, S: Middle Ob)

Die russ. Partikel $a j$ - 'und' ist im Textkorpus vor allem als Adverb 'wieder' belegt. Nur in südselkupischen Texten wird sie auch zum Ausdruck additiver Satzverbindungen (X und Y) verwendet, wie in den Beispielen (222a) und (222b). In (222a) tritt sie in beiden Funktionen (als Adverb und Konjunktion) auf.

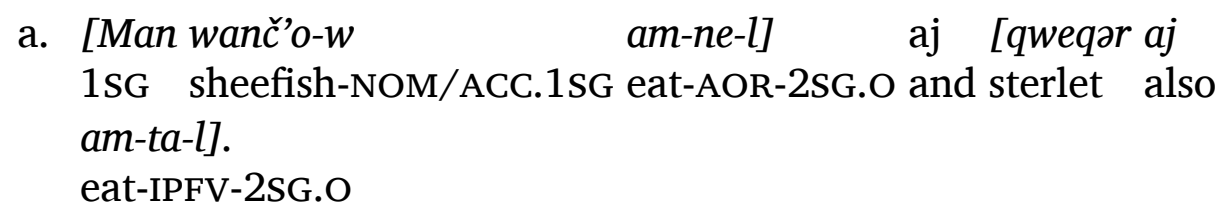

'Meinen Weißlachs hast du gegessen und einen Stör hast du auch gegessen.'

(NN_1879_Itja_flk_74, S: Chaja) 
b. [Könd tö-mba māt] aj [kwā-nd šcr-na].

horse come-PST.REP home and yard-ILL go.into-AOR.3SG

'Das Pferd kommt nach Hause und geht in den Hof hinein.'

(SEV_1980_HazelGrouse_flk_45, S: Middle Ob)

Ebenfalls additiv kann die Konjunktion $a$ - 'aber' verwendet werden wie in (223). Dort findet sich neben der Koordination mittels Konjunktion (a) auch die Juxtaposition (dritter Teilsatz n'an'n'eyit ninga).

(223) [Üde-t ne-l qup kore-yend t'ö-lla], evening-LOC.ADV woman-ADJZ person earth.house-ILL.3SG come-OPT.3SG

a [tab naj t'ö-lla], [n'an'n'e-yit ni-nga].

and 3SG also come-OPT.3SG forward-LOC stand-AOR.3SG

'Am Abend kommt die Frau in die Erdhütte und er (der Waldgeist) kommt auch und steht davor.'

(TMR_1981_Devil_flk_7, S: Middle Ob)

Wie in den nordsamojedischen auch (vgl. Wagner-Nagy 2019: 430) können die selkupischen Temporaladverben nìni, kate, nanto und undo - 'dann' sowie eine Reihe von Adverben, die aus dem Russischen entlehnten wurden, ebenfalls als koordinierende Konjunktionen fungieren. Die Adverben nanto und undo - 'dann' sind jeweils nur in einer Dialektgruppe belegt, nanto im Südselkupsichen, wie in (226), undo im Zentralselkupischen, wie in (227). Für nīni und kate - 'dann' finden sich Beispiel in beiden Dialektgruppen, wie in (224a) und (224b) bzw. (225a) und (225b). Von russischen Entlehnungen sind im Textkorpus to - 'dann', potom - 'dann' und tagda - 'dann' belegt, wie in den Beispielen (228) - (230b). Die Temporaladverben erscheinen immer satzinitial und formen kausale Konstituentensätze.

a. [Kidgul ara tadi udo-m-de kori-mba-d], [nategit sorcerer old.man right hand-ACC-3SG swing-PST.REP-3SG.O there n'ar-i-tko $\varepsilon d e-m b a] \quad$ [nind mener-til kup swamp-TRL become-PST.REP.3SG then hunt-PTCP.PRS person to-špi-nd], [le-r-i-čc-mba]. come-IPFV-INFER.3SG sing-FRQ-EP-INT.PF-PST.REP.3SG

'Der Zauberer winkte mit der rechten Hand, dort erschien ein Sumpf und der Jäger kam, er sang.' (ChDN_1983_HerosDaughter_flk_42-43, Z: Vasjugan)

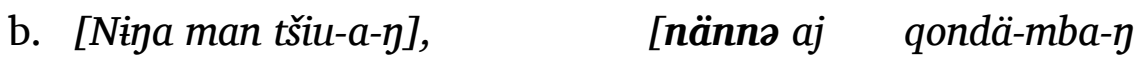
so 1SG wake.up-AOR-1SG.S then again go.to.sleep-PST.REP-1SG.S to qaro-tta]. until morning-ILL

'So wachte ich auf und schlief wieder bis zum Morgen.' (KMS_1967_EagleOwl_nar_9, S: Middle Ket') 
(225)

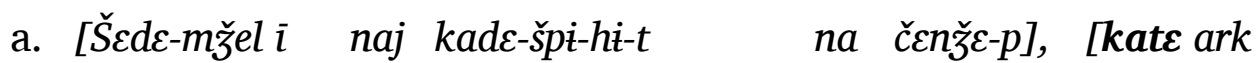
two-ORD son also tell-IPFV-AOR-3SG.O this story-ACC then other i-la šidi-r-ba-dit $\quad k \varepsilon c ̌ c i k a-\eta], \quad \ldots$ son-PL two-VBLZ-PST.REP-3PL alternating

'Der zweite Sohn erzählte die Geschichte und die anderen Söhne wiederholten sie, ...' (KIA_2014_ItjaKet_trans_80, Z: Narym)

b. $\mathrm{Nu}, \quad$ [azi-t $\quad q \bar{u}-\mathrm{mba}], \quad$ [katte azi-m-d INTERJ father-3SG die-PST.REP.3SG then father-ACC-3SG ta-mbat]. bury-PST.REP-3SG.O

'Nun, der Vater starb und sie beerdigte den Vater.' (AGS_1968_FairytaleSnake_flk_56, S: Middle Ket')

(226) [Pū-də-k mazim], [nanto ke-l'e-e-w-s]. cross-IPFV-IMP.2SG.O 1SG.ACC then tell-OPT-1SG.O-CLIT

'Setz mich über und ich erzähle es dir.' (PMP_1961_Fairytale_flk_274, S: Middle Ob)

(227) [Na-l-gup nilaži-k ora-l-ba-di heləžว hir-t woman-ADJZ-person such-ADV hold-INCH-PST.REP-3SG.O seven cow-GEN kob], [undo nika föt-pa-di].

skin then here sew-PST.REP-3SG.O

'Die Frau nahm sieben Kuhfelle und nähte sie.' (PMP_1961_Fairytale_flk_274, S: Middle Ob)

(228) [Šoyor ǐža-l-gu-k:] [Pan-ē-ž-et po-p šoyor-o-nd], [tagdā stove say-INCH-ITER-3SG.S put-EP-IPFV-IMP.2SG.O tree stove-EP-ILL then kadē-be]. tell-1SG.O

'Der Ofen sagt: Lege Holz in den Ofen und ich erzähle es dir.' (TTD_1964_WildDucks_flk_14, Z: Tym)

(229) [Čop-so-nd ema-n-otti konda-i]; [to nu-t čibe-nd maľ̆ swing-NMLZ-ILL woman-GEN-with sleep-1DU then god-GEN end-ILL forever konda-nna-i]. sleep-AOR-1DU

'Wir legten uns mit der Frau in die Wiege und schliefen für immer bis ans Ende der Zeit.'

(NN_1855_Hero4_song_170, Z/S: Tym/Middle Ob)

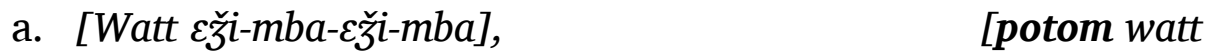
road become-PST.REP.3SG-become-PST.REP.3SG then road netu-či-mba]. NEG.EX-DRV-PST.REP.3SG
} 
'Es gab einen Weg, es gab einen und der Weg war weg.' (MNS_NN_BullSon_flk_158, Z: Narym)

b. [Teb-nan t'eli-m-na-n ipotom tel'ti-m-na-n 3SG-ADE sun-TRL-AOR-3SG.O son-3SG then sun-TRL-AOR-3SG.O ne-t]. daughter-3sG 'Ihm wurde ein Sohn geboren und ihm wurde eine Tochter geboren.' (PVD_1961_FarmAssault_flk_4, S: Chaja)

\subsubsection{Adversativ}

Zum Ausdruck adversativer Satzverbindungen werden im Textkorpus Varianten der aus dem Russischen entlehnten Konjunktion $a$ - 'aber' verwendet. Beispiele finden sich in beiden Dialektgruppen, wie in (231a).

a. [Imja-l paja İ̌̌e-p qwilit-p qwat-ku] a woman-ADJZ old.woman Itja-ACC fish-ACC catch-INFbut Itja [Ǐ̌̆e lari-mba]. be.afraid-HAB.3SG

'Die Alte (schickt) Ijta Fische fangen, aber Itja fürchtet sich.' (MNN_1977_ItjaGrandmother_flk_1, Z: Tym)

\subsubsection{Disjunktiv}

Disjunktive Satzverbindungen werden mit einer Variante der aus dem Russischen stammenden Konjunktion il'i - 'oder' ausgedrückt. In beiden Dialektgruppen sind Beispiele belegt, wie in (232a) und (232b).

a. Fu, [kaj-l kup] ali [lod tö-špi-nda]?

huh what-ADJZ person or devil come-IPFV-INFER.3SG

'Puh, geht dort ein Mensch oder ein Teufel?'

(MNS_NN_BullSon_flk_112, Z: Narym)

b. [Ta-nan qaj qum je-za-n] al'i [lōw k'ed'ig'o-yən]?

2SG-ADE what person be-PST-3SG.S or devil basket-LOC

'War in deinem Korb ein Mensch oder ein Teufel?'

(PMP_1961_ForestWoman_flk_286, S: Middle Ob)

Es konnte herausgearbeitet werden, dass die Parataxe im Selkupischen ursprünglich entweder durch ein reines Nebeneinanderstellen von Teilsätzen oder die Verwendung infiniter Verbfomen ausgedrückt wurde. Beide Möglichkeiten sind sowohl in zentralals auch in südselkupischen Texten belegt, Juxtaposition in (219a) und (219b), infinite Verbformen in Form von Konverben in (220a) - (220f). Konverben können einfach oder komplex sein und auch gemischt innerhalb eines komplexen Satzes erscheinen. Im Textkorpus lassen sich komplexe Sätze mit vier Teilsätzen belegen, Beispiel (220e) 
enthält drei konverbale Formen und eine finite Verbform.

Bereits (Helimski 1998: 576 f.) beschreibt die Verwendung von (in der Regel aus dem Russischen entlehnten) koordinierenden Konjunktionen. Sie sind in den Beispielen (221a) - (232b) beschrieben. Mit ihnen werden additive, kontrastive, adversative und disjunktive Sachverhalte ausgedrückt. Zur Wiedergabe kausaler Sachverhalte können eine Reihe von Temporaladverben verwendet werden. Sie sind in den Beispielen (224a) - (227) gezeigt. Auch dort finden sich russische Entlehnungen ((228) - (230b)). Tabelle 19 zeigt sämtliche belegte koordinierende Konjunktionen im Überblick.

Tab. 19: Koordinierende Konjunktionen im Selkupischen

\begin{tabular}{ll}
\hline Additiv & $i-$ - 'und' \\
& $a j-$ 'oder' \\
& $a-$ 'aber' \\
& $n \bar{z} n \dot{t}-$ 'dann' \\
& $k a t e-$ 'dann' \\
& nanto - 'dann' \\
& undo - 'dann' \\
& to - 'dann' \\
& potom - 'dann' \\
& tagda - 'dann' \\
\hline Kontrastiv & $i-$ 'und' \\
\hline Adversativ & $a-$ 'aber' \\
\hline Disjunktiv & $i l ' i-$ 'oder' \\
\hline
\end{tabular}

\subsection{Subordination}

Bei der Subordination (auch Hypotaxe) sind die beiden Teilsätze syntaktisch nicht gleichwertig. Vielmehr ist der Konstituentensatz (auch „Nebensatz“) in den Matrixsatz (auch „Hauptsatz“) eingebettet. Im Selkupischen wird Subordination ebenfalls ursprünglich mittels Juxtaposition und infiniten Verbalformen (Konverben, Infinitiv) ausgedrückt (vgl. Helimski (ebd.: 576 f.)). Des Weiteren gibt es eine Reihe subordinierender Konjunktionen, die aus dem Russischen entlehnt wurden.

\subsubsection{Juxtaposition}

Typischerweise werden auch subordinierte Sätze ohne verbindendes Element nebeneinandergestellt, wie in den Beispielen (233a) - 233g). Dabei kann der Matrixsatz dem Komplementsatz vorausgehen wie in (233a) - (233c) oder vice versa wie 
in (233d) - 233g). Untersuchungen Im Textkorpus lassen vermuten, dass mit der Satzfolge MATRIXSATZ + KONSTITUENTENSATZ Konditionalsätze (Beispiele (233a) (233c)) gebildet werden, während die umgekehrte Satzfolge Komplementsätze (Beispiele (233d) - (233f)) oder Temporalsätze (Beispiel (233g)) ergibt. Aufgrund der geringen Datenlage lassen sich keine weitergehenden Analysen durchführen.

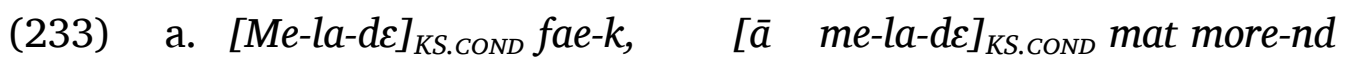
give-OPT-3SG.O good-ADV NEG giv-OPT-3SG.O 1SG sea-ILL amde-l-b-la-ge.

sit.down-INCH-HAB-OPT-1SG.S

'Wenn er sie freigibt, ist es gut, wenn er sie nicht freigibt, stoße ich ihn ins Meer.'

(MNS_NN_BullSon_flk_67, Z: Narym)

b. [Tab čwesse manče-že-lla $]_{K S . C O N D}$, ulyo tab-i-m aj

3SG backward look-IPFV-OPT.3SG ice 3SG-EP-ACC again

kur-o-l-ti.

run-EP-INCH-3SG.O

'Wenn sie zurückschaut, wird das Eis sie wieder forttragen.'

(ChDN_1983_GirlAndIce_flk_22, Z: Vasjugan)

c. [T'āza-lži-me-nd-ža-l] $]_{K S . C O N D}$ Pönegessa tö-nža $i \quad \bar{a} m-\check{z} a$

noise-TR-COND-FUT-2SG.O Pönege come-FUT.3SG and eat-FUT.3SG

št.

2SG.ACC

'Wenn du lärmst, wird Pönege kommen und dich fressen.'

(TFF_1967_KolobokkaPoenegessa_flk_7, S: Middle Ob)

d. Üče-d'e tane-tì-mba [ün onže

child-3sG know-IPFV-PST.REP.3SG water himself

čaže-li-mba] $]_{K S . C O M P L}$.

go.out-RES-PST.REP.3SG

'Das Kind dachte, dass das Wasser (das Boot) selbst trägt.'

(ChDN_1983_Nikita_flk_16, Z: Vasjugan)

e. Asa tänu-a-i [madur e-k ali čamu-a-n] $]_{K S . C O M P L}$.

NEG know-AOR-1DU hero be-3SG.S or NEG.EX-AOR-3SG.S

'Wir wissen nicht, ob es ein Held ist oder nicht.'

(NN_1855_Hero1_song_335, Z/S: Tym/Middle Ob)

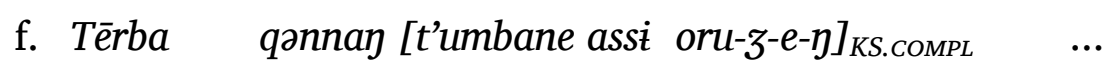

think.3sG dog wolf NEG force-INSTR-be-3sG.S

'Der Hund denkt, dass der Wolf nicht stark ist ...'

(BNN_1971_DogSearchedMistress_flk_25, S: Middle Ket')

g. A tan tü-r'-e-na-nž, [ma-nan'e paniz-o-wi-m

but 2SG cry-FRQ-EP-AOR-2sG.S 1SG-ADE scythe-EP-1SG-ACC 
ko-lt'e-nza-l'] $]_{\text {KS.TEMP. }}$

sight-INCH-IPFV-2SG.O

'Aber du weinst, wenn du meine Sense erblickst.'

(PMP_1961_ForestWoman_flk_33, S: Middle Ob)

\subsubsection{Infinite Verbalformen}

Wie bei der Parataxe zählt auch bei der Hypotaxe die Juxtaposition neben der Verwendung infiniter Verbformen zu den ursprünglichen Mitteln, subordinierte Sachverhalte auszudrücken. Helimski (1998: 576 f.) beschreibt neben Nominalisierungen auch Partizipien und Konverben. Im Textkorpus lassen sich lediglich Konverben und der Infinitiv als infinite Verbformen belegen. Nominalisierungen kommen im gesamten Korpus nicht in komplexen Sätzen vor. Partizipien fungieren lediglich auf Phrasenebene als Modifikator von NPs (vgl. Kapitel 2, Abschnitt 2.1). Anders als in nordsamojedischen Sprachen (vhl. hierzu die enzischen Beispiele bei Siegl (2013: 459) und die nganasanischen Beispiele bei Wagner-Nagy (2019: 453 ff.)) zeigen Partizipien im Selkupischen weder Kasus- noch Numeruskongruenz mit dem Subjekt des Matrix- oder Konstituentensatzes. In Übereinstimmung mit Dixon (2010b: 316) formen Partizipien im Selkupischen daher keine Relativsätze, sie sind Adjektiven zuzuordnen (N.G. Kuznecova 1995: 204). In den zentralselkupischen Texten ist die Adjektivisierung auch formal zu erkennen: Sämtliche Partizipien tragen dort das Adjektivsuffix - $l$. In südselkupischen Texten sind Formen mit und ohne Adjektivsuffix vorhanden.

\subsubsection{Subordinierende Konverbsätze}

Im komplexen Satz können Konverben im Selkupischen verwendet werden, um Subordination auszudrücken. Aus einer europäischen Sichtweise ist das sogar deren Hauptaufgabe, indem es definiert wird als „a nonfinite verb form whose main function is to mark adverbial subordination. Another way of putting it is that converbs are verbal adverbs, just like participles are verbal adjectives" (Haspelmath 1995: 3). Konverben als subordinierendes Prädikat kommen im Textkorpus vor allem in südselkupischen Texten vor. Mit einem Süd-Nord-Gefälle lassen sie sich auch in zentral- und nordselkupischen Dialekten belegen. Erste Korpusanalysen zeigen (zumindest) für die südlichen Dialekte einen eindeutigen Zusammenhang zwischen der konverbalen Form (einfach bzw. komplex), dem Tempus des Matrixsatzes und dem Adverbialsatz: Temporalsätze der Gleichzeitigkeit werden mit einfachen Konverben und einem „Non-Past-Tempus“(Präsens, Aorist) gebildet wie in den Beispielen (234a) und (234b). Temporalsätze der Vorzeitigkeit werden mit komplexen Konverben und einem „Past-Tempus“(reportatiyes Präteritum, Präteritum, Aorist) gebildet wie in den Beispielen (234c) und (234d). Vereinzelt finden sich auch Konditionalsätze wie in (234e) und Finalsätze wie in (234f) und (??). Erstere werden mit Hilfe einer 
Konverben und der finiten Verbform im Präsens oder Futur gebildet. Letztere sind durch ein der finiten Verbform nachgestelltes Konverb gekennzeichnet wie in (234f).

(234) a. [Onek mōyne čač-le $]_{\text {KS.TEMP.SIM }}, l \bar{\varepsilon}-r-a-k$. myself home run-CVB sing-FRQ-AOR-3SG.S

'Während ich nach Hause ging, sang ich.' (TTD_1964_Squirrel_flk_2, Z: Tym)

b. [Mat amde-le $]_{K S . T E M P . S I M}$, saľ̌e-po passe-že-k, ille 1SG sit-CVB stump-tree break-IPFV-3sG.s down kuan-na-k; go.away-AOR-3SG.S

'Während ich sitze, brach der Fußboden ein, ich ging hinab.' (NN_1855_Hero1_flk_36-39, Z/S: Tym/Middle Ob)

c. $[\text { Awit-t, azi-t } \quad \boldsymbol{q} w \bar{\varepsilon} l-\check{c} \text {-lebe }]_{K S . T E M P . A N T} t \bar{o}-m b a-d i t$. mother-3SG father-3SG fish-TR-CVB come-PST.REP-3PL

'Die Mutter (und) der Vater kamen, nachdem sie gefischt hatten.' (ILP_1981_ItjaOneEyedDevil_flk_10, S: Middle Ob)

d. ... [nagur po-gandä kü̈da-l'ewl'e] $]_{K S . T E M P . A N T} q u-m b i-s$. three year-ILL.3SG be.ill-CVB die-HAB-PST.3SG

'Sie starb, nachdem sie drei Jahre lang krank war.' (AGS_1968_Fairytale_flk_11, S: Middle Ket')

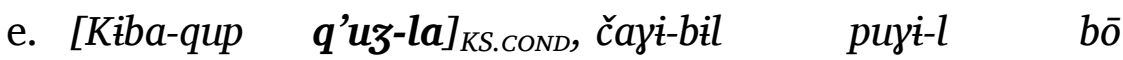
small.person urinate-CVB dry-PTCP.PST rotten-ADJZ tree püna-lč-ku-t natideli, n'ala-l puyi-l bō tē fill-INT.PF-ITER-3SG.O there raw-ADJZ rotten-ADJZ tree away qotta-l-gu-t. throw-INCH-ITER-3SG.O

'Wenn das kleine Kind einnässt, schüttet sie trockene Holzstückchen dorthin (in die Wiege), die nassen Holzstückchen wirft sie weg.' (ILP_1981_ItjaOneEyedDevil_flk_10, S: Middle Ob)

f. Ma tö-mba-ut [čabe-r-če-le nena-nde-tko] $]_{K S . F I N}$. 1PL come-PST.REP-1PL marry-FRQ-TR-CVB sister-OBL.3SG-TRL 'Wir sind gekommen, die Schwester zu freien.' (NN_1855_Hero3_song_252, Z/S: Tym/Middle Ob)

\subsubsection{Subordinierter Infinitivsatz}

Mit Hilfe des Infinitivs können komplexe Sätze gebildet werden. Im Textkorpus können ausschließlich finale Sachverhalte belegt werden. Dabei erscheint die finite Verbform immer satzfinal, die infinite Form unmittelbar davor. Im Konstituentensatz kann ein direktes Objekt lexikalisch overt ausgedrückt werden, wie die Akkusativobjekte 
in (235a) und (235d) oder die Nominativ-Objekte in (235b) und (235c). Letztere enthalten darüber hinaus elliptische infinite Teilsätze (post-p-ču-gu - 'um zu fasten' in (235b) sowie ügo-l-gu čwasse - 'um zurückzubringen' in (235c)), in denen die finite Verbform nicht noch einmal wiederholt wird.

a. Tab-e- $\gamma$ mad'o-nde kwin-ba- $\gamma$, [weš $-a-p$ 3SG-EP-DU taiga-ILL go.away-PST.REP-3DU.s cedarwood.cone-EP-ACC takki-l-gu kwin-ba- $]_{\text {KS.FIN }}$. collect-INCH-INF go.away-PST.REP-3DU.S

'Sie gingen in den Wald, gingen, um Zedernzapfen zu sammeln.' (ChDN_1983_ItjaStayedAlone_flk_7, Z: Vasjugan)

b. Ogon ws'o [nom nom-tə-gu $]_{K S . F I N} q w a j a-q-w a \overline{-} d \partial t \quad[\text { post-ə-ču-gu }]_{K S . F I N}$. earlier all god god-CAP-INF go-ITER-AOR-3PL fasting-EP-TR-INF 'Früher gingen alle, um zu Gott zu beten (und) zu fasten.' (SAA_1971_ThreeSisters_flk_8, Z: Narym)

c. qwel-lej [waక̌’o-t taka kire-l-gu $]_{K S . F I N}$ [ügo-l-gu go.away-IMP.1DU meat-3SG away pull.off-INCH-INF drag-INCH-INF čwasse $]_{\text {KS.FIN }}$. backward

'Laß uns gehen, um das Fleisch abzuziehen (und) es zurückzubringen.' (PAV_NN_HowICatchBear_nar_73, Z: Tym)

d. ... ondi-t omdi-za-dat, [pa-la-m-dit s'ila-mb'i-gu $]_{K S \text {.FIN }}$. himself-PL sit.down-PST-3PL knife-PL-ACC-3PL sharpen-HAB-INF

'Sie setzten sich, um ihre Messer zu schleifen.' (ILP_1981_ItjaOneEyedDevil_flk_10, S: Middle Ob)

e. $[S o g o-n \check{e} e-g u]_{K S \text {.FIN }}$ töu-a-k. ask.for-IPFV-INF come-AOR-3SG.S

'Ich bin gekommen, um zu fragen.'

(NN_1855_Hero1_song_24, Z/S: Tym/Middle Ob)

\subsubsection{Konjunktionalsätze}

Mit Hilfe von subordinierenden Konjunktionen werden im Selkupischen unterschiedliche Konstituentensätze geformt. Ebenso wie die koordinierenden sind auch die subordinierenden Konjunktionen im Selkupischen vorrangig aus dem Russischen entlehnt. Im Textkorpus sind l'i - 'ob' ( < russ. li), štobì - 'dass' ( < russ. štobi), bitta 'als ob' ( < russ. bita), jes'li bzw. ešl'e - 'wenn' ( < russ. jesli), kagda - 'wenn' ( < russ. kogda), pakka - 'während' ( < russ. poka), pus't' - 'dass' (< russ. < pust'), xot' - 'obwohl' (< russ. xot'), čem - 'als' (< russ. čem), bi - 'wenn' (<russ. bi) und puskaj 'dass' ( $<$ russ. puskaj) sowie die selkupischen Interrogativpronomen qanduy - 'wie', kuča - 'wo', ku - 'wohin' und kuti் - 'wer' belegt. Neben Komplementsätzen können auch Adverbialsätze mit Konjunktionen eingeleitet werden. 


\subsubsection{Komplementsätze}

Komplementsätze fungieren als das obligatorische Argument (sowohl Objekt, als auch Subjekt) des Verbs im Matrixsatz. Im Gegensatz zum Adverbialsatz, der den Matrixsatz modifiziert, vervollständigt ein Komplementsatz den Matrixsatz grammatisch. Im Selkupjschen können Komplementstrategien mittels Juxtaposition (Beispiele (233d) - (233f) in Abschnitt 5.2.1) oder konjunktionalen Komplementsätzen umgesetzt werden. Als Konjunktion fungieren die (selkupischen) Interrogativpronomen qanduך 'wie', kuča - 'wo', ku - 'wohin' und kuti - 'wer'. Prototypisch nehmen Verben wie „sehen“, „hören“, „wissen“, „glauben“ und „mögen“ Komplementsätze in Objektfunktion an (Dixon 2010b: 371). Das Konzept „wollen“ wird im Selkupischen ebenfalls lexikalisch realisiert. Daher nimmt das Modalverb kiga - 'wollen' ebenfalls einen Komplementsatz als Objekt, wie in (237a) und den (237c). Beide Beispiele enthalten neben dem Komplementsatz einen weiteren subordinierten Teilsatz - einen Finalsatz, der mit einem Infinitiv (Beispiel 237a) bzw. einem Supinumi (Beispiel (237c)) ausgedrückt wird.

Die Beispielsätze (236a) - (236d) enthalten Komplementsätze, die mit Interrogativpronomen eingeleitet sind. Der Komplementsatz folgt typischerweise dem Matrixsatz, wie in (236a), (236b) und (236d), er kann ihm aber auch vorausgehen, wie in (236c). Das Interrogativpronomen erscheint in der Regel zu Beginn des Komplementsatzes. Im Textkorpus sind nur Komplementsätze belegt, die als Objekt des Matrixsatzes erscheinen. Ob auch andere Komplemente (Subjekt, Prädikat) mittels Komplementsatz ausgedrückt werden können, kann anhand der Datenbasis nicht ermittelt werden.

a. Šoyor, šojor, tat ā tenu-a-l, [kuča-d n'āb-la stove stove 2SG NEG know-AOR-2SG.O where-ADV duck-PL waše-mbā-dst $]_{K S . \text { сомPL }}$ [mat čemn'a-l'ika-m $\mathbf{k u}$ fly-PST.REP-3PL 1SG brother-DIM-ACC where qwan-dā-det $]_{\text {KS.COMPL }}$ ? go.away-TR-3PL

'Ofen, Ofen, weisst du nicht, wohin die Enten geflogen sind, wohin sie meinen Bruder gebracht haben?'

(TTD_1964_WildDucks_flk_13, Z: Tym)

b. Tat tEnu-a-l [mat kud e-nda-k] $]_{K S . C O M P L}$ ?

2SG know-AOR-2SG.O 1SG who be-INFER-1SG.S

'Weisst du, wer ich bin?'

(TTD_1964_CalfHare_flk_25, Z: Tym)

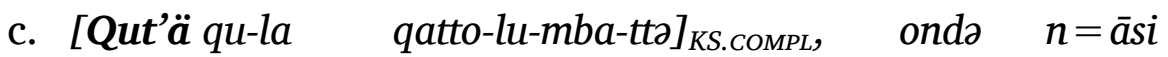
where person-PL disappear-RES-PST.REP.3PL himself emph $=$ NEG tinno-wo-t. know-AOR-3SG.S

${ }^{1}$ Das Beispiel ist der einzige Beleg für ein Supinum im Textkorpus, es ist vor allem im Nordselkupischen belegt. 
'Wohin die Menschen verschwunden sind, weiß er selbst nicht.' (AGS_1968_FairytaleSnake_flk_35, S: Middle Ket')
[qaj-nuך al'c'ika
and forget-INT.PF-3SG.O what-TRL grandmother
d. ... i ao-lžz-t,
t'itta-r-ba] $]_{\text {KS.COMPL }}$.
walk-FRQ-PST.REP.3SG
'... und vergaß, wie die Großmutter gegangen war.'
(TFF_1967_ItjaAldigaNet_flk_7, S: Middle Ob)

Das Modalverb kiga - 'wollen' verlangt typischerweise ein Objektkomplement. Neben der üblichen Wortfolge, in der die finite Verbform satzfinal erscheint, wie in (237b) und (237d), ist auch die umgekehrte Wortfolge belegt, wie in (237a) und (237c).

(237)
a. ... naššakit na ne-l
qup uruy mad'o-yonde kiga-k
then this woman-ADJZ person very taiga-ILL.3SG want-3SG.S
$[\mathrm{kwen}-\mathrm{gu}]_{\text {KS. COMPL}}$.
go.away-INF
'... dann wollte diese Frau sehr (häufig) in den Wald gehen.'
(ChDN_1983_TaleBrownies_flk_26, Z: Vasjugan)
b. Tabi [am-gu $]_{\text {KS. сомPL }}$ kige-li-mba.
3SG eat-INF
want-RES-PST.REP.3SG
'Er wollte sie fressen.'
(KFN_1967_Bear_flk_7, Z: Tym)
c. Sap [nādi-gu $]_{\text {KS. СомРL }}$ kige-lit-mba.
egret get.married-INF want-RES-PST.REP.3SG
'Der Reiher wollte heiraten.'
(TMR_1981_SapSapiha_flk_4, S: Middle Ob)
d. Na qum qigi-nba [tü-m t’äda-gu $]_{K S . C O M P L}$.
this person want-PST.REP.3SG fire-ACC burn-INF
'Dieser Mensch wollte ein Feuer anzünden.'
(AGS_1968_FairytaleSnake_flk_37, S: Middle Ket')

Die aus dem Russischen entlehnte Konjunktion što - 'was' wird im Textkorpus verwendet, um Komplementsätze als Objekt auszudrücken. Die_Konjunktion leitet den Komplementsatz konsequent ein, wie in den Beispielsätzen (238a) - (238d).

(238) Objektsatz

a. N'an'a eži-m mekka me-le-l, [što kwan-na-nž] $]_{\text {KS. сомРL }} \ldots$ sister word-ACC 1SG.DAT give-OPT-2SG.O what go.away-AOR-2sG.S

'Schwester, gib mir dein Wort, dass du gehst ...'

(SAI_1984_StoryAboutLifeLong_nar_24, Z: Narym)

b. Mat tär-ba-k, [što mašik ab-lā-de čumbne-la] $]_{\text {KS. сомPL }}$.

1SG think-PST.REP-1SG.S what 1SG.ACC eat-OPT-3PL wolf-PL 
'Ich dachte, dass die Wölfe mich fressen würden.' (TTD_1964_CalfHare_flk_6, Z: Tym)

c. Man $i$ čara-u onen, što tan kworg mena ik 1SG and say-1SG.O myself what 2SG bear 1.DAT.SG NEG nat-ka, a u3 man teya as nat-ča-u. vex-IMP.2SG.S and already 1SG 2SG.DAT NEG vex-FUT-1SG.O

'Ich sage (zu mir) selbst, dass du Bär, mich nicht belästigt hast, und ich dich auch nicht belästigen werde.'

(NN_1879_Isyrgul_flk_92, S: Lower Chaya)

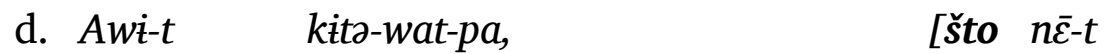

mother-3SG get.frightened-DETR-PST.REP.3SG what daughter-3SG

tar-ze $\quad a z i-m b a]_{K S . C O M P L}$.

hair-INSTR become-PST.REP.3SG

'Die Mutter erschrank, dass ihre Tochter behaart war.'

(TMR_1981_Devil_flk_26, S: Middle Ob)

\subsubsection{Adverbialsätze}

Im Gegensatz zu Komplementsätzen modifizieren bzw. ergänzen Adverbialsätze den Matrixsatz. Ihr Vorhandensein ist syntaktisch nicht nötig. Sie beschreiben den Hintergrund einer Situation oder einer Handlung genauer und gehören damit, wie Adverbien, zu den Adjunkten. Anhand des Textkorpus lassen sich Beispiele für Finalsätze, Kausalsätze, Komparativsätze, Konditional- und Konsekutivsätze sowie Modal- und Temporalsätze belegen. Teilweise werden die Satztypen mit Hilfe originär russischer Konjunktionen gebildet, teilweise fungieren selkupische Pronomen als Marker.

\subsubsection{Finalsatz}

Finalsätze gehören zu den Adverbialsätzen und beschreiben den Zweck, die Absicht oder das Ziel eines im Matrixsatzes genannten Sachverhaltes. Im Textkorpus sind Finalsätze vor allem mit infiniten Konstruktionen belegt (siehe Abschnitt 5.3.2.2). Unter russischem Einfluss gibt es außerdem konjunktionale Finalsätze, die mit der aus dem Russischen stammenden Konjunktion štobi் - 'dass' ( < russ. štobí) eingeleitet sind. Der Finalsatz erscheint in der Regel nach dem Matrixsatz, wie in (239a), (239c) und (239d), die umgekehrte Reihenfolge ist jedoch auch möglich, wie in (239b). Im Gegensatz zu infiniten Konstruktionen (Beispiele (235a) - (235e) in Abschnitt 5.3.2.2), in denen das Subjekt des Finalsatzes identisch mit dem des Matrixsatzes ist, unterscheiden sich die Subjekte der eingeleiteten Finalsätze von denen des Matrixsatzes.

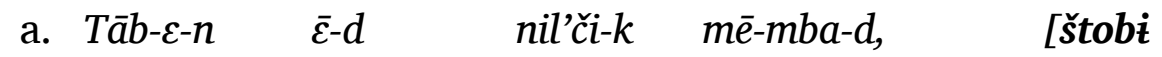
3SG-EP-GEN father-3SG such-ADV do-PST.REP-3SG.O that nē-d nāyor po-d čāmže-tko éč-le wark-ni $]_{K S . F I N}$. daughter-3SG three year-LOC.ADV frog-TRL become-CVB live-AOR.3SG 
'Ihr Vater hatte ihr das angetan, sodass seine Tochter drei Jahre lang in einen Frosch verwandelt lebte.'

(TTD_1964_Frog_flk_89, Z: Tym)

b. [Čtobi ka-t-ka nüžc-p paža-l-gu hir-e-paja-n] $]_{K S . F I N}$, that winter-ILL-? grass-ACC chop-INCH-INF cow-EP-woman-DAT nado nüз̆e-p pača-l-gu šed meh-t. necessary grass-ACC chop-INCH-INF two barn-3SG

'Damit die Kuh im Winter Heu hat, muss man zwei Heuschober Gras mähen.'

(TMN_1994_Cow_nar_11, Z: Narym)

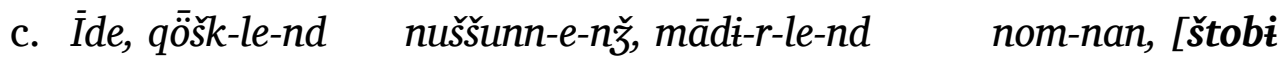
Itja go-OPT-2SG.S sky-EP-ILL ask.for-OPT-2SG.S god-ADE that pöd-nc $]_{\text {KS.FIN }}$ ! warm.up-AOR.3SG

'Itja, geh in den Himmel und bitte Gott, dass es wärmer wird!' (ILP_1981_ItjaGrandmother_flk_3, S: Middle Ob)

d. Üte-t nar [štob as qoj qu-la qüt'i-ja-dat $]_{K S . F I N}$, qoj let.go-3sG.o so that NEG rich person-PL be.ill-AOR-3PL rich qu-la qou-ne-dat. person-PL rich-VBLZ-3PL

'Es ging so, dass die armen Menschen krank wurden und die reichen Menschen (noch) reicher.'

(KKN_1971_IvanAndGod_flk_34, S: Upper Ket')

\subsubsection{Kausalsatz}

Kausalsätze beschreiben den Grund für einen Sachverhalt näher, der im Matrixsatz benannt wird. Beispiele für Kausalsätze sind im Textkorpus nur selten vorhanden. In allen Beispielen folgt der Konstituentensatz dem Matrixsatz und ist ohne Konjunktion an diesen angeschlossen. Stattdessen erfolgt eine Markierung im Matrixsatz mit Hilfe des Adverbs nano - 'dann' bzw. der Translativform des Demonstrativpronomens na - 'dieser', die unmittelbar nach dem lexikalisch overten Subjekt erscheint (nach dem Personalpronomen der 1. Person in (240a) und (240b)) bzw. an der Position, an der das lexikalisch overte Subjekt erscheinen würde (satzinitial in Beispiel (240c)). Ähnliche Beispiele finden sich auch im Nordselkupischen. Genauere Aussagen lassen sich aufgrund der wenigen Textbeispiele nicht treffen.

(240) a. A mat na-tqo tö-mba-k, [man-nāni ì-m and 1SG this-TRL come-PST.REP-1SG.S 1SG-ADE son-1SG nādi-gu kiga] $]_{\text {KS.KAUS }}$. get.married-INF want.3SG

'Und ich bin deshalb gekommen, (weil) mein Sohn heiraten möchte.' (SEV_1980_Hazelgrouse_flk_22, S: Middle Ob) 


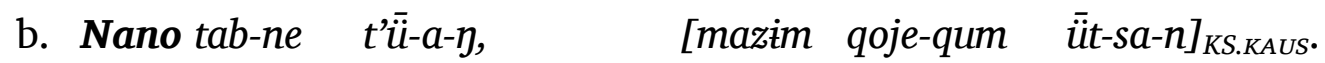
then 3SG.DAT come-AOR-1SG.S 1SG.ACC rich-person send-PST-3SG.S 'Ich komme deshalb zu ihm, (weil) mich ein reicher Mann geschickt hat.' (PMP_1961_Fairytale_flk_189, S: Middle Ob)

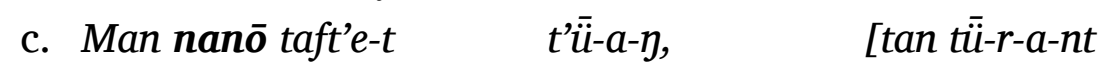

1SG then here-LOC.ADV come-AOR-1SG.S 2SG cry-FRQ-AOR-2SG.S

oqir-i-le $]_{\text {KS.KAUS. }}$

one-EP-CVB

'Ich komme deshalb hierher, (weil) du allein weinst.'

(PMP_1961_ForestWoman_flk_310, S: Middle Ob)

\subsubsection{Komparativsatz}

Eine prototypische Komparativkonstruktion vergleicht zwei Partizipienten hinsichtlich des Grades einer ihnen zugeordneten Eigenschaft. Das Selkupische verwendet neben der Postposition tare - 'wie' (Kapitel 2.7) und Kopulakonstruktionen mit ablativischen Komplementen (Kapitel 4) die Interrogativpronomen qundar und kanduk 'wie' (und ihre Varianten) sowie die aus dem Russischen entlehnten Konjunktionen čem - 'als' (< russ. čem) und kak - 'wie' ( < russ. kak - 'wie'), um Komparation auszudrücken. Letztere bilden komplexe Sätze, wie in den Beispielen (241a) - (241c). In (241a) ist die „brauchen“-Konstruktion nach russischem Muster (Dativ + Partikel nado - 'nötig') mit der aus Turksprachen entlehnten Partikel k'er'eg'ey - 'nötig' gebildet. In Beispiel $241 \mathrm{c}$ ist der Komparativsatz in den Matrixsatz eingeschoben.

a. Ne-j-gum

$j \bar{\imath}-\gamma a-t$

t'anqo-pti-t

wes

woman-ADJZ-person take-AOR-3SG.o spread.out-CAUS-3SG.O all

[kuntar tab-ne k'er'eg'eך] $]_{\text {KS. комРAR }}$.

how 3SG-DAT/ALL necessary

'Die Frau nahm (es), breitete alles aus, wie sie es brauchte.'

(PMP_1961_ForestWoman_flk_246, Middle Ob)

b. Tab, nid'ek, me-mba-t [kanduk paja

3SG girl do-PST.REP.3SG how old.woman

kura-ľ̆e-mba-d] $]_{K S . K O M P A R}$

command-INT.PF-PST.REP-3SG.O

'Sie, das Mädchen, tat (so), wie die Alte (es ihr) befohlen hatte ...' (ChDN_1983_GirlAndIce_flk_31, Z: Vasjugan)

c. İd'ä-n ald'əgə-t nā kätə-m [kuddar $]_{\text {KS.KOMPAR }} \bar{e}-s$ Itja-GEN grandmother-3SG this gut-ACC how be-PST.3sG qwēs'e pel-le tāda-r-a-t.

backward put-CVB bring-FRQ-EP-3sG.O

'Itjas Großmutter legte diesen Magen (wieder) so zurück, wie er war.' (NN_1913_Itja_flk_71, S: Middle Ob) 
Die aus dem Russischen stammenden Konjunktionen čem - 'als' und kak - 'wie' bilden ebenfalls Komparativsätze. Im Textkorpus findet sich čem vor allem in übersetzten Texten, wie in den Beispielsätzen (243a) und (243b), kak hingegen auch in selkupischen, wie in (242c). Die Konjunktionen leiten den Konstituentensatz stets ein, in der Regel erscheint der Komparativsatz nach dem Matrixsatz, eine Ausnahme bildet (242a). In (242b) und (242b) werden komparative Sachverhalte mit der Bedeutung 'so als ob' durch die Kombination von kak - 'wie' und s'oramno bzw. s'o rawno - 'ganz gleich' (< russ. vse rawno - 'ganz gleich') gebildet.

(242) a. [Kak n'äj šenušna pišnaq muzēe-špā] $]_{\text {KS.KOMPAR, n'il'd'i-k }}$ how bread wheat fluffy cook-IPFV.3SG such-ADV or-we-špa-t.

grow-DRV-IPFV-3PL

'Wie prächtiges Weizenbrot reift, so wachsen sie.' (SDP_1964_FairytaleBlackZar_trans_61, Z: Narym)

b. Or-wa-špa inne da inne alojō-dat [kak s'oramno

grow-DRV-IPFV.3SG up and up boat-3PL how it.doesn't.matter

onžว $m \bar{e}-g a-z e-s ̌ p a]_{K S . K O M P A R}$.

himself do-RFL-DRV-IPFV.3SG

'Ihr Schiff wird höher und höher, als ob es sich selbst baute.'

(SDP_1964_FairytaleBlackZar_trans_1158, Z: Narym)

c. Nil'd'z'i- $\eta$ waryi- $\eta$ t'ali-mpa-n, pel-galik, [kak s'o rawno

such-ADV big-ADV talk-HAB-3SG.s friend-CAR how it.doesn't.matter

koc'e qum t'äli-mi-n-t. $]_{\text {KS.KOMPAR }}$.

much person talk-DRV-AOR-3PL

'Er spricht allein so laut, als ob viele Menschen sprächen.'

(PMP_196_BodylessHead_flk_33, S: Middle Ob)

(243) a. Tabə päl-yalk ödä-r-lā tī žăda wes öt-ku-lde, 3SG friend-CAR sew-FRQ-FUT.3SG 2DU even all sew-ITER-INT.PF.3SG

[č'em tī šedagwöjget qup öde-r-na-lt $]_{K S . K O M P A R}$.

than 2DU twelve person sew-FRQ-AOR-2PL

'Er wird allein für euch nähen, er näht alles besser, als ihr zwölf Menschen es genäht hättet.'

(SDP_1964_FairytaleBlackZar_trans_279, Z: Narym)

b. Pil'esa-n ira $n$ ō̄ $\eta \quad$ qo-nži-r-ni-t $t$ [čem $s \bar{o}$ side eye-GEN old.man good-ADV sight-IPFV-FRQ-AOR-3SG.o than good šitta sai qum] $]_{\text {KS.KOMPAR. }}$

two eye person

'Der einäugige Alte sieht besser als ein zweiäugiger Mann.'

(KMS_1967_Antosja_trans_18, S: Middle Ket') 


\subsubsection{Konditionalsatz}

Kontitionalsätze sind ebenfalls Adverbialsätze. Sie formulieren Bedingungen, unter denen die Handlung im Matrixsatz erst stattfinden kann. Im Textkorpus kommen Konditionalsätze nur selten vor. In Beispiel (234e) aus dem Abschnitt 5.2.2.1, das hier als Beispiel (244) wiederholt wird, bildet eine Konverbkonstruktion einen Konditionalsatz. Darüberhinaus können mit Hilfe der ursprünglich russischen Konjunktionen (to) l' $i$ - 'ob' ( < russ. (to) li), bi - 'wenn' ( < russ. bi), jes'li bzw. ešl'e - 'wenn' ( < russ. jesli) und kagda - 'wenn' (< russ. kogda) Konditionalsätze gebildet werden, die in den Beispielsätzen (245a) - (247) gezeigt sind. Mit einer Ausnahme (Beispiel (246a)), erscheint der Konditionalsatz vor dem Matrixsatz.

(244) [Kiba-qup q'uz-la $]_{K S . C O N D}$, čaji-bil puyi-l bō small.person urinate-CVB dry-PTCP.PST rotten-ADJZ tree püna-lč-ku-t natideli, n'ala-l puyi-l bō tē fill-INT.PF-ITER-3SG.O there raw-ADJZ rotten-ADJZ tree away qotta-l-gu-t. throw-INCH-ITER-3SG.O

'Wenn das kleine Kind einnässt, schüttet sie trockene Holzstückchen dorthin (in die Wiege), die nassen Holzstückchen wirft sie weg.'

(ILP_1981_ItjaOneEyedDevil_flk_10, S: Middle Ob)

a. [Mat tanu-p-ni-m $\boldsymbol{b} \dot{\boldsymbol{i}}]_{K S . C O N D}, \check{s} \bar{t} w \quad \bar{a}$

1SG know-HAB-AOR-1SG.O if ashes NEG kotta-l-b-ni-m bi. throw-INCH-HAB-AOR-1SG.O if

'Wenn ich das gewusst hätte, hätte ich die Asche nicht weggeworfen.' (MNS_1984_BrotherSister_flk_78, Z: Narym)

b. [Okkir-saj-i lōz bi tö-nc $]_{K S . C O N D,}$ mat okkir-saj-i lōz one-eye-ADJZ devil if come-AOR.3SG 1SG one-eye-ADJZ devil qum-ne qwan-nc- $\eta$. person-DAT go.away-AOR-1SG.S 'Wenn ein einäugiger Teufel käme, würde ich ihn heiraten.' (ILP_1981_ItjaOneEyedDevil_flk_4, S: Middle Ob)

c. [Ezel'i man bi tun-ni-w] $]_{K S . C O N D}, \quad k u z a=n n e j$ as t'apto-ni-w. if $1 \mathrm{SG}$ if know-AOR-1SG.O when = EMPH NEG promise-AOR-1SG.O 'Wenn ich es gewusst hätte, hätte ich das niemals versprochen.' (PMP_1966_BoyDevil_flk_23, S: Middle Ob)

(246) a. Mì ombì inne tata-lu qìlō-ute, [ešli ellāa $]_{K S . C O N D}$. 1PL now up bring-1DU pit-PROL if live.3SG 'Wir werden ihn jetzt aus der Grube hochbringen, wenn er noch lebt.' (SDP_1964_FairytaleBlackZar_trans_997, Z: Narym) 
b. [Jes'liw tan täppi-la-m qottä mita-r-e-nža-l if 2SG 3SG-PL-ACC on.ones.back achieve-FRQ-EP-FUT-2SG.O kündi-māt-täa $]_{K S . C O N D}$ man mi-nža-m tejga okkə künde-m. horse-house-ILL 1SG give-FUT-1SG.O 2SG.DAT one horse-ACC 'Wenn du sie in den Stall treibst, werde ich dir ein Pferd geben.' (AGS_1968_FairytaleSnake_flk_61, S: Middle Ket')

(247) [Kagda tï̈-r-a-nt $]_{K S . C O N D}$ megajl'e as upว-l-š-q-wa-nt. when cry-FRQ-AOR-2SG.S 1SG.DAT NEG listen-INCH-US-ITER-AOR-2SG.S 'Wenn du weinst, hörst du mich nicht.' (PMP_1961_ForestWoman_flk_116, S: Middle Ob)

\subsubsection{Konsekutivsatz}

Ein Konsekutivsatz ist ein Adverbialsatz, der eine Folge des im Matrixsatz genannten Sachverhaltes benennt. Im Textkorpus ist ein Beispiel aus einem übersetzten Text belegt, das in (248) gezeigt ist. Der Konsekutivsatz ist dem Matrixsatz nachgestellt und mit der Translativform des Demonstrativpronomens na - 'dieser' eingeleitet. Zusätzlich erfolgt im Matrixsatz eine Markierung mittels des Adverbs nìləžik - 'solcher'.

(248) Itte nilaži-k or-he tu-špi-ha lab-he, [na-tko

Itja such-ADV force-INSTR row-IPFV-AOR.3SG oar-INSTR this-TRL

tab-i-t ando-nd koče-k üt kam-ni-mba] $]_{K S . K O N S E Q}$.

3SG-EP-GEN boat-ILL much-ADV water pour-AOR-PST.REP.3SG

'Itja ruderte mit solcher Kraft, sodass viel Wasser in sein Boot lief.'

(KIA_2014_ItjaKet_trans_10, Z: Narym)

\subsubsection{Konzessivsatz}

Konzessivsätze sind ebenfalls Adverbialsätze und drücken Umstände aus, die der Aussage des Matrixsatzes entgegenstehen und diese daher unerwartet aussehen lassen. Wie für den Konsekutivsatz ist auch für den Konzessivsatz lediglich ein Beispiel im Korpus belegt. Es ist in (249) gezeigt und wird mit der russischen Konjunktion xot' 'obwohl' ( < russ. xot') eingeleitet. Das Beispiel stammt ebenfalls aus einem übersetzten Text. Der Konzessivsatz geht dem Matrixsatz voraus.
[Xot' me saqe-l'e-kā-l-bo-t
$n \bar{u}-1 l a-m$

although 1PL stick.through-RES-ITER-DRV-PST.REP-1PL hole-PL-ACC

pil'd'u-kka-l'a-sä] $]_{K S . K O N Z}$, üt t'äk ä-ku-s topa-n illo-qän.

cloth-DIM-PL-INSTR water fast be-ITER-PST.3SG leg-GEN ground-LOC

'Obwohl wir die Löcher mit Lappen zustopften, war das Wasser schnell unter den Füßen.'

(MMP_1964_Lgov_trans_124, S: Upper Ket') 


\subsubsection{Temporalsatz}

Adverbiale Temporalsätze beschreiben eigenständige Handlungen und ordnen sie der Handlung des Matrixsatzes zeitlich zu: vorzeitig, gleichzeitig oder nachzeitig. Im Textkorpus werden Temporalsätze typischerweise mit Konverben (siehe Beispiele (234a) (234d) Abschnitt 5.2.2.1 oben) oder mittels Juxtaposition (Abschnitt 5.2.1) gebildet. Darüber hinaus können Temporalsätze mit Hilfe der russischen Konjunktion pakka'(solange) bis' ( < russ. poka) gebildet werden. Sie ist nur in südselkupischen Texten belegt. Die Beispiele (250a) und (250b) zeigen Temporalsätze, die zeitlich gleichzeitig (250a) bzw. vorzeitig (250b) zur Handlung des Matrixsatzes stehen.

a. $n u \quad i$ warqa-n [pakamas tab-i-nan n'ūš je-ya-n] $]_{K S . T E M P .}$ INTERJ and live-3SG.S until 3SG-EP-ADE grass be-AOR-3SG.S

'Nun, und sie lebte, solange sie Heu hatte.'

(PMP_1966_BodylessHead_flk_99, S: Middle Ob)

b. [Pakka qāra-m ütt-i-t pen-ži-zit-t $]_{K S . T E M P}$

until fish.trap-ACC water-EP-ILL put-IPFV-PST-3SG.O

merge-n-ni $\quad$ i t'ōwa-ľ̌i $\eta$.

wind-VBLZ-AOR.3sG and snow-IPFV-3sG.S

'Bis sie die Reuse ins Wasser legte, kam Wind auf und es begann zu schneien.'

(KMS_1966_TwoSisters_flk_9, S: Middle Ket')

\subsubsection{Relativsätze}

Relativsätze sind Konstituentensätze, die in den Matrixsatz eingebettet sind und als syntaktischer Modifikator einer NP des Matrixsatzes fungieren. Formal komplexe Relativsätze kommen äußerst selten vor, typischweise fungieren Partizipien als Modifikator einer Nominalphrase (vgl. Kapitel 4). In den Beispielen (251a) - (251c) formen Partizipien auch formal komplexe Sätze. Sie modifizieren jeweils eine NP im Matrixsatz und erscheinen, wie alle Modifikatoren im Selkupischen, unmittelbar vor dem Kopfnomen (lo - 'Teufel' in (251a), qula - 'Menschen' in (251b)). Im Beispielsatz (251c) steht der Relativsatz nach der NP na tibequm - 'dieser Mann'. Anders als beim attributiven Gebrauch der Partizipien, folgt die interne Struktur der Relativsätze der für das Selkupische beschriebenen Wortfolge SOV (Helimski 1998: 576), indem die direkten Objekte (mat tcbn'am - 'mein Bruder.NOM/ACC.1SG' in (251a), mazim '1SG.ACC' in (251b), qulam - 'Menschen.ACC' in (251c)) dem Partizip vorausgehen. Unter russischem Einfluss finden sich in den Texten darüberhinaus Beispiele für eingeleitete Relativsätze. Die Interrogativpronomen kod bzw. kut - 'wer' und qaj - 'was' fungieren in den Beispielen (252a) - (252c) als Relativpronomina. Dabei kann der eingeleitete Relativsatz in den Matrixsatz eingeschoben werden wie in Beispiel (252b). 
(251)

a. Na [mat tebn'a-m am-bil $]_{K S . R E L}$ lo

this 1SG brother-NOM/ACC.1SG eat-PTCP.PST devil

tö-špi-nda.

come-IPFV-INFER.3SG

'Dieser Teufel kommt, der meinen Bruder gefressen hat.'

(MNS_NN_BullSon_flk_126, Z: Narym)

b. [Mazim pōne t'aš-ku-nd'i $]_{K S . R E L}$ qu-la čāż-n-dat.

1SG.ACC outward throw-ITER-PTCP.PRS person-PL go-AOR-3PL

'Die Menschen gehen, die mich hinausgeworfen haben.'

(PMP_1961_ForestWoman_flk_304, S: Middle Ob)

c. A na tibe-qum, [qu-la-m pu-t-ka-ndi $]_{K S . R E L}$ [ando-qin and this man-person person-PL-ACC cross-DRV-ITER-PTCP.PRS boat-LOC

pal'd'i-ndi $]_{K S . R E L}, t a b-\dot{i}-m$ ant meza-l-gu-t t'ör-o-n

go-PTCP.PRS 3SG-EP-ACC boat pull-INCH-ITER-3SG.O lake-EP-GEN

saj-gint.

eye-LOC.3SG

'Und dieser Mann, der die Menschen hinüberbringt, der im Boot fährt, ihn zieht das Boot in die Mitte des Sees.'

(PMP_1961_Fairytale_flk_110, S: Middle Ob)

(252)

a. Nïlaži-k $\varepsilon$-a koče-k bar, kuza-t madur

such-ADV be-AOR.3SG much-ADV time when-LOC.ADV hero

čače-špi-hi-t kwa-he madur-la-p, [kod

shoot-IPFV-AOR-3SG.O arrow-INSTR hero-PL-ACC who

$n \dot{i}$ - $\boldsymbol{i}$-ha-dit $\quad k \varepsilon-t \quad$ par-o-git $t_{K S . R E L}$.

stand-DRV-AOR-3PL river-GEN top-EP-LOC

'So passierte es mehrere Male, wenn der Held einen Pfeil auf die Helden schoss, die am Ufer standen.'

(KIA_NN_GiantZobel_trans_57, Narym)

b. Sūru-p [qaj qwal-lā-dí-s $s_{K S . R E L}$ na sūru-p

wild.animal-ACC what catch-OPT-3PL-CLIT this wild.animal-ACC

tādi-kku-zā-dit tab-ni.

bring-ITER-PST-3PL 3SG-DAT/ALL

'Die Tiere, die sie fingen, diese Tiere brachten sie ihr.'

(ILP_1981_StonyOldWoman_flk_5, Middle Ob)

c. Medi- $\eta \quad$ qoj qu-nä, [kut tab-i-m ü

achieve-3SG.S rich person-DAT/ALL who 3SG-EP-ACC send-PST-3SG.O

$s \ddot{u}-n e_{K S . R E L}$.

dragon-DAT/ALL

'Er erreichte den reichen Menschen, der ihn zum Drachen geschickt hatte.'

(PMP_1961_Fairytale_flk_281, Middle Ob) 


\subsubsection{Kombination von Koordination und Subordination}

In komplexen Sätzen können Koordination und Subordination kombiniert werden. Beispiele dieser Art sind im Korpus nur vereinzelt belegt, wie das komplexe Beispiel in (253). Der Konstituentensatz drückt einen konjunktionalen Sachverhalte aus, in ihn eingebettet ist der Komplementsatz kwatku čežalguk - 'er kann nicht jagen', der mit dem negativen Hilfsverb čeža gebildet ist. Des Weitern sind in den Konjunktionalsatz die Nominalphrasen astep - 'Hirsch' und peqqap - 'Elch' eingebettet, die durch Juxtaposition koordinierend verbunden sind. An den Matrixsatz nada kwallede peqqop - 'muss er einen Elch jagen' sind mittels Juxtaposition weitere fünf Teilsätze angehängt, wobei der unmittelbar folgende Teilsatz qajl'emil peqqal'ikap - 'irgendeinen kleinen Elch' elliptisch ist und eine Ergänzung zum Teilsatz davor darstellt.

(253) [Mene-r-til qup mad'o-yit [aste-p, peqqa-p kwat-ku] $]_{\text {KS.комРL }}$ hunt-FRQ-PTCP.PRS person taiga-LOC deer-ACC elk-ACC kill-INF

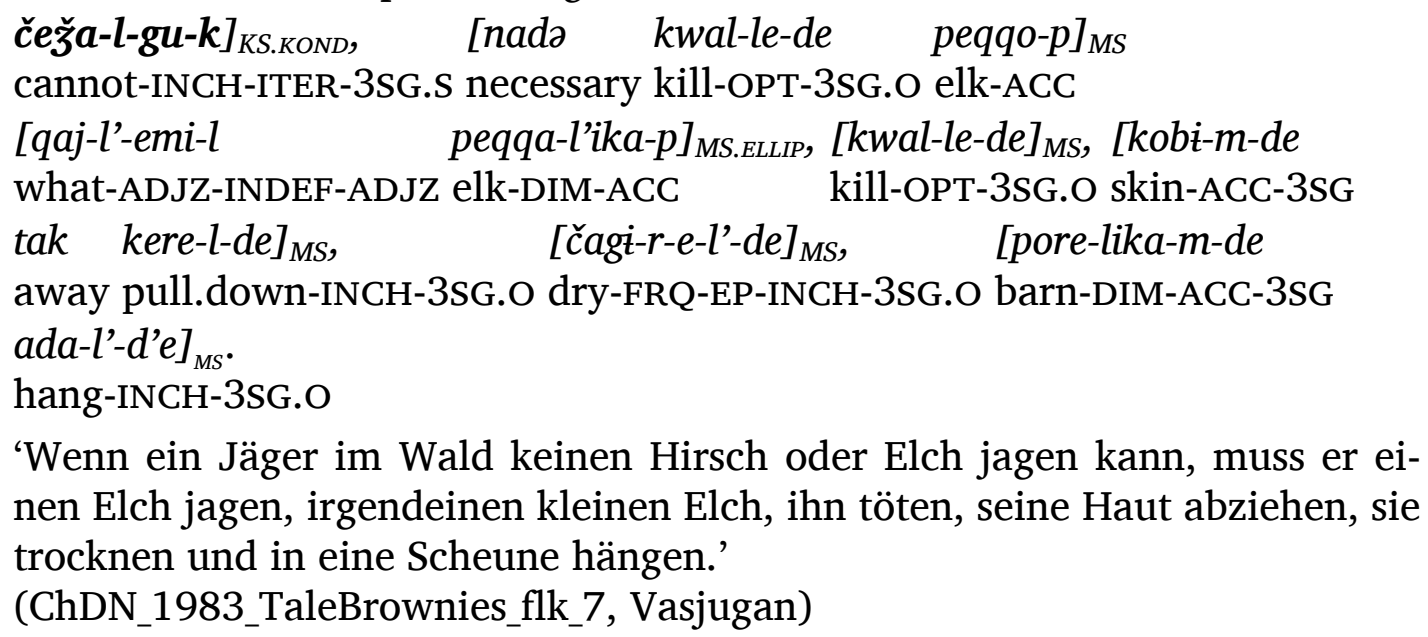

\subsection{Negation komplexer Sätze}

Zu Beginn des Kapitels 5 wurde bereits beschrieben, dass ein komplexer Satz gebildet wird, indem zwei oder mehr Teilsätze durch Koordination oder Subordination miteinander verbunden werden. In Bezug auf negierte Sätze unterscheidet Dixon (2012: 90f.) eine multiple Markierung von Negation von einer Doppelnegation. Bei ersterer werden mehrere oder alle Satzglieder innerhalb eines Teilsatzes („clause“) jeweils einzeln negiert, die Negation hat dabei jeweils nur Skopus über die Konstituente, die sie negiert. Bei letzterer kommen verschiedene Negationen mit jeweils eigenem Skopus innerhalb eines Satzes vor (z. B. jeweils eine Negation im Matrix- und im Konstituentensatz), der Skopus der Negation umfasst also das gesamte Satzgefüge. Im Textkorpus finden sich Beispiele sowohl für die multiple Negation als auch für die Doppelnegation. Im Folgenden werden negierte koordinierte und subordinierte Sätze betrachtet. Dabei kann der Skopus der Negation sich jeweils auf nur einen oder auf mehrere Teilsätze beziehen bzw. auf den komplexen Satz als Ganzes. 


\subsubsection{Koordination negierter Sätze}

Koordinierte Teilsätze werden mittels Juxtaposition, infiniten Verbformen (Konverben) sowie Konjunktionen miteinander verbunden (siehe auch 5.1). Mit Hilfe der Negationswörter aus Tabelle 8 können ein oder mehrere Teilsätze negiert werden. Im Nordselkupischen findet sich eine Doppelnegation, wenn ein Interrogativpronomen in der Rolle eines Komplements negiert wird (vgl. auch Abschnitt 4.4.5 oben).

\subsubsection{Negation bei Juxtaposition}

Negierte asyndetisch koordinierte Teilsätze sind im Korpus häufig belegt, ebenso wie ihr affirmatives Korrelat. Dabei wird typischerweise eine Sequenz aufeinanderfolgender Satzteile ausgedrückt (siehe auch Kapitel 5.1.2) wie in den Beispielsätzen (254a) (254e). Teilsätze können aber auch adversativ verbunden sein wie in (255a) - (255c) oder korrelativ wie in (256a). Letzteres wird mit Hilfe einer Doppelnegation ausgedrückt, indem in beiden Teilsätzen das grammtische Subjekt mit der Negationspartikel $n$ 'i und das Prädikat mit der Negationspartikel $\bar{a}$ negiert werden. In (256b) sind die Teilsätze sowohl additiv als auch korrelativ verbunden. Im Gegensatz zu Beispiel (256a) wird die Korrelativität jedoch nicht mit einer doppelten Negation ausgedrückt. In den koordinierten Sätze sind neben Beispielen, in denen die Negation ausschließlich im letzten Teilsatz erfolgt (Beispiele (254a) - (254c) bzw. (255a) - (255c)), auch solche mit multipler Negation belegt wie in (254d) und (254e).

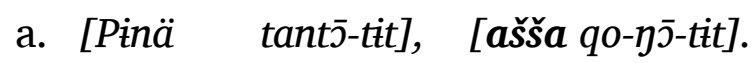
outwards go.out-3PL NEG find-AOR-3PL

'Sie gehen hinaus, sie finden (sie) nicht.' (BIV_1941_KonMytyke_flk_29, N: Taz)

b. [Olo-d Ella], [haj-e-de ko-mbi-lí-mba], [aga čEnča]. head-3SG live.3sG eye-EP-3sG sight-HAB-RES-PST.REP NEG say.3sG 'Der Kopf lebt, die Augen sehen, (aber) sie spricht nicht.' (ChDN_1983_Pora_flk_20, Z: Vasjugan)

c. [Tab-it $t$ pe-mba-dit], [pe-mba-dit], [aga 3SG-EP-PL look.for-PST.REP.3PL look.for-PST.REP-3PL NEG ko-mba-dit]. sight-PST.REP-3PL

'Sie suchten, suchten, sahen ihn nicht.' (ChDN_1983_Pora_flk_20, Z: Vasjugan)

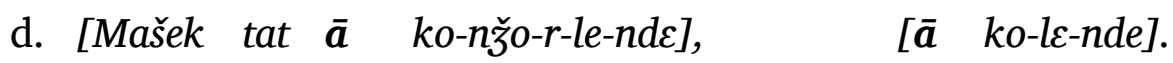
1SG.ACC 2SG NEG sight-IPFV-FRQ-OPT-2SG.S NEG find-OPT-2SG.S 'Du wirst mich nicht sehen und nicht finden.' (MNS_NN_BullSon_flk_102, Z: Vasjugan)

e. [Pl'ejpō az üngว-ľs-q-wa-tt], [qwaja-m mat pitchfork NEG listen-INT.PF-ITER-AOR-3SG.O yard-ACC house 
an-a-la as ünga-lš-q-wa-tt], [qud=nej as mouth-EP-PL NEG listen-INT.PF-ITER-AOR-3PL who $=$ EMPH NEG üngu-lza-ku- $\eta \quad n \bar{e}-j-q u-n e]$. listen-INT.PF-ITER-3SG.S woman-ADJZ-person-DAT/ALL

'Die Heugabel hört nicht, die Hoftore hören nicht, niemand hört auf die Frau.'

(PMP_1966_BodylessHead_flk_95, S: Middle Ob)

a. [Qaqli-sä ašša qaqlì-tta], [čanak tol'či-sä sūrin'-n'a]. sledge-INSTR NEG sledge-VBLZ.3SG only ski-INSTR hunt-AOR.3SG 'Er fährt nicht mit dem Schlitten, sondern jagt auf Skiern.' (AAI_1973_Okyle_flk_6, N: Taz)

b. [Pi-n d'onక̌o-n qannaך muda-n'e], [kum assi night-GEN half-LOC.ADV dog bark-IPFV.3SG person NEG woza-ku- $\eta$ ]. get.up-ITER-3SG.S

'Um Mitternacht bellt der Hund, (aber) der Mensch steht nicht auf.' (BNN_1971_DogSearchedMistress_flk_45, S: Middle Ket')

c. [N'o-kka qonde-za-n], [qənnay assi kondu-l-ba] [mūt-l'e hare-DIM sleep-PST-3SG.S dog NEG sleep-INCH-HAB.3SG bark-CVB olda- $\eta$ ]. begin-3SG.S

'Das Häschen schläft, der Hund schläft nicht ein, (sondern) er fängt an zu bellen.'

(BNN_1971_DogSearchedMistress_flk_9, S: Middle Ket')

(256)

a. [N'ī čapte-t $\overline{\bar{a}}$ kad-le], [n'ī haj-o-n-d $\overline{\boldsymbol{a}}$ NEG tale-3SG NEG tell-OPT.3SG NEG eye-EP-GEN-3SG NEG qo-nža-r-d-le]. sight-IPFV-FRQ-TR-OPT.3SG

'Weder kann ein Märchen (darüber) erzählt werden, noch kann das Auge es sehen.' (TTD_1964_Frog_flk_61, Z: Tym)

b. [Kude-m=nej ass aps-tu-ku-s-ta], [era-ga-ne, who-ACC $=$ EMPH NEG eat-TR-ITER-PST-3SG.O old.man-DIM-DAT/ALL paja-ga-ne qai-m=ne az me-ku-t]. old.woman-DIM-DAT/ALL what-ACC = EMPH NEG give-ITER-3SG.O 'Er füttert niemanden und gibt weder einem Alten noch einer Alten etwas.'

(PMP_1961_Fairytale_flk_13, S: Middle Ob) 


\subsubsection{Negierte koordinierte Konverbsätze}

In negierten komplexen Konverbsätzen wird ausschließlich der Teilsatz negiert, der eine finite Verbform enthält, typischerweise ist das der letzte Teilsatz. Sämtliche Beispiele stammen aus dem Ket'-Subdialekt des südselkupischen Subkorpus, nord- und zentralselkupische Beispiele finden sich nicht. Wie im einfachen Satz auch erscheint die Negationspartikel $a$ šša bzw. eine Variante unmittelbar vor dem satzfinalen Prädikat, wie in (257b) und (257c), in (257a) befindet sich das direkte Objekt in satzfinaler Stellung.

a. [Na qum t'ü-u-l'e], [teb assa por-a-či-t saľ̌zi-m]. this person cry-AOR-CVB 3SG NEG burn.down-EP-TR-3SG.O stump-ACC 'Dieser Mensch weinte, (aber) er brannte den Baumstumpf nicht nieder.' (AGS_1968_FairytaleSnake_flk_40, S: Middle Ket')

b. [Üd'i-mbi-l'e] [üd'i-mbi-l'e], [kuw-a-m assi ko-wə-t]. send-HAB-CVB send-HAB-CVB person-EP-ACC NEG find-AOR-3SG.O

'Er lief, lief, (aber) er fand den Menschen nicht.' (BNN_1971_DogSearchedMistress_flk_38, S: Middle Ket')

c. [Loz'e qwe-lewl'e] [t'ita as tü-qu- $\eta$ ]. devil go.away-CVB here NEG come-ITER-3SG.S 'Loz ging weg, (und) kam nie wieder hierher.' (KKN_1971_Itja_flk_79, S: Upper Ket')

\subsubsection{Negierte koordinierte Konjunktionalsätze}

Komplexe Sätze können mit Hilfe von koordinierenden Konjunktionen gebildet werden. Die Konjunktionen sind typischerweise aus dem Russischen übernommen, wie in den Beispielen (258a) und (258b). Dort werden die Teilsätze mit $i$ - 'und' additiv bzw. mit il'i - 'oder 'disjunktiv verbunden. In beiden Beispielen ist jeweils der zweite Teilsatz negiert, in (258b) enthalten die beiden Teilsätze außerdem ein identisches Prädikat, der negierte Teilsatz ist daher elliptisch und enthält lediglich die Negationspartikel assa.

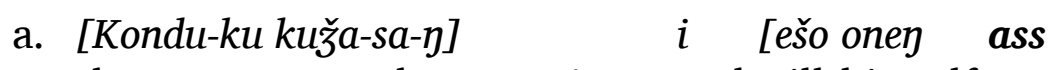
sleep-INF go.to.sleep-PST-1sG.S and still himself NEG qondo-l-bi-sa- $\eta]$. sleep-INCH-HAB-PST-1sG.S

'Ich legte mich hin, um zu schlafen und schlief noch nicht.' (PMP_1961_Fairytale_flk_20, S: Middle Ob)

b. [Qos kundä čāzi-ku-mba], il'i [assa]. DEF long go-ITER-PST.REP.3SG or NEG

'So ging er lange oder nicht.' (AGS_1968_FairytaleSnake_flk_31, S: Middle Ket') 


\subsubsection{Subordination negierter Sätze}

Bei der Verbindung zweier Teilsätze mittels Hypotaxe ist der Konstituentensatz (KS) in den Matrixsatz (MS) eingebettet. Ebenso wie affirmative Sätze (vgl. Kapitel 5.2) können auch negierte Teilsätze sowohl mit Hilfe infiniter Verbformen (Konverb und Infinitiv) als auch mittels Konjunktionen subordiniert werden.

\subsubsection{Negierte subordinierte Konverbsätze}

Im Gegensatz zu koordinierten Konverben können subordinierte Konverben auch negiert werden. In den Beispielen (259a) und (259b) erscheinen die Konverben kwilla 'weggehen.CVB' und čadil'e - 'anzünden.CVB'im Matrixsatz und sind mit der StandardNegationspartikel aga bzw. aya negiert. In (259c) erscheint das affirmative Konverb ükendil'ewl'e - 'Hut.aufsetzen.CVB' umgekehrt im temporalen Konstituentensatz. Negiert ist wiederum der Matrixsatz. Das Vorkommen von Konverben im Matrixsatz in den Beispielsätzen (259a) und (259b) ist ungewöhnlich. Die genaue Funktion des Konverbs kann aufgrund fehlender Beispiele gegenwärtig nicht erklärt werden. Ebenfalls ungeklärt bleibt die Frage, ob eine Negation sowohl im Konstituenten- als auch im Matrixsatz möglich ist, oder ob Konstituentensätze negiert werden können, die eine konverbale Form enthalten. Hier kann erst die weitere Forschung Antworten geben.

a. Man ne-m tekka aga kwil-la, [tan

1SG daughter-NOM/ACC.1SG 2SG.DAT NEG go.away-CVB 2SG

ara-ma-nd] $]_{\text {KS.KAUS }}$.

old-thing-2sG.S

'Meine Tochter bekommst du nicht, (weil) du alt bist.'

(ChDN_1983_HerosDaughter_flk_36, Z: Vasjugan)

b. Tat tü̈-m aya čadi-l'e [tat indiga-y mazik

2sG fire-ACC NEG light-CVB 2sG daughter.in.law-DIM 1sG.ACC

n'aja-ľ̆ı $-\gamma a]_{\text {KS.KAUS. }}$

get.angry-INT.PF-AOR.3SG

'Du kannst kein Feuer anzünden, (weil) deine Schwiegertochter mich erzürnt hat.'

(ChDN_1983_MistressOfFire_flk_61-62, Z: Vasjugan)

c. [Nö-j qob'i üku-n-də üke-n-dì-l'ewl'e $]_{K S . T E M P}, n \ddot{o}-j$

hare-ADJZ skin hat-GEN-3SG hat-VBLZ-IPFV-CVB hare-ADJZ

noppi-la-m as ser-b'i-nda- $\eta$.

glove-PL-ACC NEG dress-HAB-IPFV-3SG.S

'Nachdem sie die Mütze aus Hasenfell aufgesetzt hatte, hatte sie die Hasenhandschuhe noch nicht angezogen.'

(KKN_1971_WhiteHeadedWoman_flk_17, s: Middle Ket') 


\subsubsection{Subordinierter Infinitivsatz}

Mit Hilfe des Infinitivs können komplexe Sätze gebildet werden, die auch negiert stets finale Sachverhalte ausdrücken (Kapitel 5.3.2.2 für affirmative Infinitivsätze). Einzelne Beispiele finden sich in allen Dialektgruppen, negiert ist in allen Beispielen der Matrixsatz, der dem Konstituentensatz vorausgehen kann wie in (260c), nachgestellt sein kann wie in (260d) oder in den Konstituentensatz eingeschoben erscheint wie in (260a) und (260b). Bis auf eine Ausnahme erscheint die finite Verbform satzfinal.

(260) a. omti-j-qo näja-i-im-tìnä pon

horn-ADJZ-head daughter-PL.POSS-ACC-OBL.2SG outwards

[sōntir-qa] $]_{K S . F I N}$ aša ütti-mmì-mpa-t.

play-INF NEG let.go-HAB-PST.REP-3SG.O

'Der Zar lässt seine Töchter nicht hinausgehen, um zu spielen.'

(SNS_1966_Markincha_flk_3, N: Taz)

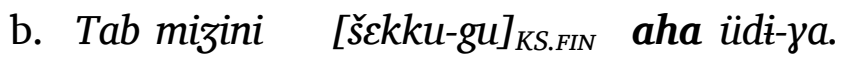

3SG 1DU.ACC spend.night-INF NEG let.go-AOR.3SG

'Er lässt uns nicht herein, um zu übernachten.'

(ChDN_1983_MotherInLaw_nar_3, Z: Vasjugan)

c. Loy-o-t tab-i-p or-o-p-t aya mede-nži-d

devil-EP-PL 3SG-EP-ACC force-EP-ACC-3SG NEG become-IPFV-3SG.O

[nindi tak hari-l-gu $]_{K S . F I N}$.

here away bind-INCH-INF

'Die Teufel hatten nicht genug Kraft, um ihn hier loszubinden.'

(ChDN_1983_Nikita_flk_37, Z: Vasjugan)

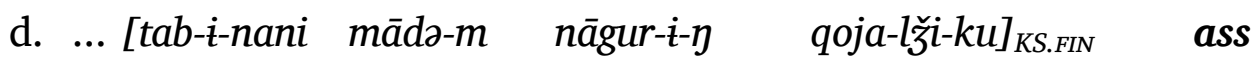

3SG-EP-ADE house-ACC three-EP-ADV surround-INT.PF-INF NEG

mide-nži-t.

achieve-FUT-3SG.O

'... (dass) er nicht schafft, sein Haus dreimal hineinzutun.'

(PMP_1961_Fairytale_flk_197, S: Middle Ob)

\subsubsection{Negierter Komplementsatz}

Das obligatorische Argument eines Verbs im Matrixsatz kann mit Hilfe eines Komplementsatzes wiedergegeben werden. Alle Beispiele im Korpus zeigen Objektkomplemente. Ob auch andere Komplemente (Subjekt) ausgedrückt werden können, kann anhand der Datenbasis nicht ermittelt werden. Negiert wird stets der Matrixsatz, mit der Negationspartikel ašša bzw. einer Variante. Beispiele für negierte Komplementsätze finden sich im Korpus nicht. Der Komplementsatz kann mit einer aus dem Russischen entlehnten subordinierten Konjunktion eingeleitet werden wie in (261a) und (261b) oder mit einem Interrogativpronomen, wie in (261d). Auch die Juxtaposition von Matrix- und Komplementsatz ist möglich, wie in Beispiel (261c). 
a. Imaqota ašša tEnì-m-i-ti, [qaj tom-ni-tī $]_{K S . C O M P L}$. old.woman NEG know-DRV-EP-3SG.O what say-AOR-3DU.O

'Die Alte versteht nicht, was sie sagen.' (BEP_1973_Fat2_flk_20, N: Taz)

b. Mat a tenu-ha-p, [qaj-p mēka me-špu-gu $]_{K S . с о м P L}$. 1SG NEG know-AOR-1SG.O what-ACC 1SG.DAT do-IPFV-INF 'Ich weiß nicht, was ich tun soll (wrtl.: was mir zu tun ist).' (TTD_1964_CalfHare_flk_5, Z: Tym)

c. Man as tunu-za-w, [ma-nan mat-kən qiba i-w 1SG NEG know-PST-1SG.O 1SG-ADE house-LOC small son-1SG t'éli-mí-tda $]_{K S . C O M P L}$. sun-VBLZ-IPFV.3SG

'Ich wusste nicht, dass bei mir zu Hause mein kleiner Sohn geboren ist.' (PVD_1966_BoyDevil_flk_20, S: Chaja)

d. [... az ünse-l'de-t] [kundar İd'ä qur-o-ni- $\eta]_{K S . C O M P L}$. NEG listen-INT.PF-3SG.O how Itja run-EP-AOR-3SG.S '... er hört nicht, wie Itja weglief.' (PMP_1967_AboutItja_flk_68, S: Middle Ob)

Das Modalverb kiga - 'wollen' erscheint typischerweise mit einem Infinitiv als Objektkomplement. Negiert wird die finite Verbform (in dem Fall das Modalverb) mit der Negationspartikel ašša bzw. einer Variante. In der Regel erscheint das Modalverb satzfinal wie in (262a), (262b) und (262e). Die umgekehrte Reihenfolge wie in (262c) und (262d) ist jedoch ebenfalls belegt.

In Beispiel (263) wird das nur in den südselkupischen Ket'-Dialekten belegte Modalverb $\bar{\varepsilon} s s u$ 'sich sehnen, verlangen, wollen'2', verwendet. Es verlangt ebenfalls ein Objektkomplement, hier ist es der Infinitiv (qaptigu) - 'löschen'.

$$
\begin{aligned}
& \text { a. ... [qum-ō-ql-m aps-ti-qo }]_{K S . C O M P L} \text { ašša kikka. } \\
& \text { person-EP-DU-ACC eat-TR-INF NEG want.3SG } \\
& \text { '... sie möchte die Leute nicht beköstigen.' } \\
& \text { (BEP_1973_Fat2_flk_5, N: Taz) }
\end{aligned}
$$

2Bykonja (2005: 312) übersetzt die Form mit 'захотеть' (zaxotet') - 'wollen' ins Russische. 
d. Tayi t'ör-o-n tibe-nt ass kige-la- $\eta$ [tü-gu andi-ze $]_{K S . с о м P L}$. lower lake-EP-GEN end-ILL neg want-OPT-3SG.S row-INF boat-INSTR

'Zum unteren Ende des Sees möchte ich nicht mit dem Boot fahren.'

(PMP_1967_AboutItja_flk_79, s: Middle Ob)

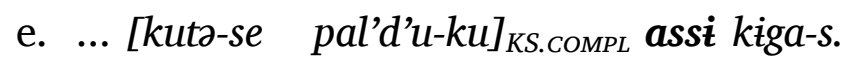
who-COM go-INF

NEG want-PST.3SG

'... er wollte mit niemandem gehen (befreundet sein).'

(BNN_1971_EyesEars_flk_7, S: Middle Ket')

(263) Tep [qapti-gu $]_{\text {KS.сомPL }}$ as es as na-நo.

3SG extinguish-INF NEG hanker.for.3SG NEG this-TRL

'Er möchte es nicht dafür löschen.'

(KKN_1971_IvanAndGod_flk_22, S: Upper Ket')

In allen drei Dialektgruppen ist das negative Hilfsverb tača bzw. čed'a, čeža oder ted'a - 'nicht können' belegt. Wie in den anderen samojedischen Sprachen auch ist es semantisch nicht vollständig leer. In sämtlichen Beispielen folgt das negative Hilfsverb dem Infinitiv. Supinformen, wie sie Wagner-Nagy (2011: 113f.) für das Nordselkupische beschreibt, sind im Korpus nicht belegt.

$$
\begin{aligned}
& \text { a. ... }[\text { sača-lti-qa }]_{\text {KS.комPL tača-l-pa-ti. }} \\
& \text { pull.out-INCH-DRV-INF cannot-INCH-HAB-3SG.O } \\
& \text { '... er kann (ihn) nicht herausziehen.' } \\
& \text { (MIV_1977_Icha_flk_9, N: Upper Taz) }
\end{aligned}
$$

b. Qäli-t [ponä tanti-qo $]_{\text {KS.КОМРL }}$ tača-l-n̄̄-tit. Nenets-PL outward(s) go.out-INF cannot-INCH-AOR-3PL

'Die Nenzen konnten nicht auf die Straße hinausgehen.' (KMP_1971_NyomalPorky_flk_34, N: Taz)

c. Tab-i-t $t$ [tü-p čadi-gu $]_{K S \text {.комРL }}$ čed'a-l-ba-dit. 3SG-EP-PL fire-ACC light-INF cannot-INCH-HAB-3PL

'Sie können kein Feuer anzünden.' (ChDN_1983_MistressOfFire_flk_38, Z: Vasjugan)

d. ... a [piri-m watti-gu $]_{\text {KS. KOMPL }}$ ted'a-l-bi-t. but stature-ACC lift-INF cannot-INCH-HAB-3SG.O

'... aber er kann nicht aufstehen (wrtl.: seine Statur nicht hochheben).' (TMR_1981_AboutItja_flk_25, S: Middle Ob)

\subsubsection{Negierter Adverbialsatz}

Anders als Komplementsätze ergänzen bzw. modifizieren Adverbialsätze den Matrixsatz. Nicht für alle in Kapitel 5 belegten Adverbialsatztypen findet sich auch ein negiertes Korrelat. Neben Konditionalsätzen können Finalsätze, Temporalsätze, 
Konsekutiv- sowie Kausalsätze als Teil komplexer Satzgefüge belegt werden. In den Beispielen ist dabei teilweise der Konstituentensatz negiert, teilweise der Matrixsatz. Nicht belegt werden können Beispiele, in denen sowohl Konstituenten- als auch Matrixsatz negiert sind.

\section{Negierter Finalsatz}

Finalsätze beschreiben den Zweck, die Absicht oder das Ziel eines im Matrixsatzes genannten Sachverhaltes. Während sich für affirmative Finalsätze im Korpus auch infinite Konstruktionen belegen lassen (vgl. 5.2.3.3), gibt es lediglich ein Beispiel für einen negierten Finalsatz. Im zentralselkupischen Beispiel (265) ist der Finalsatz mit der aus dem Russischen stammenden Konjunktion čtob - 'dass' eingeleitet und mit der Negationspartikel $\bar{a}$ negiert.

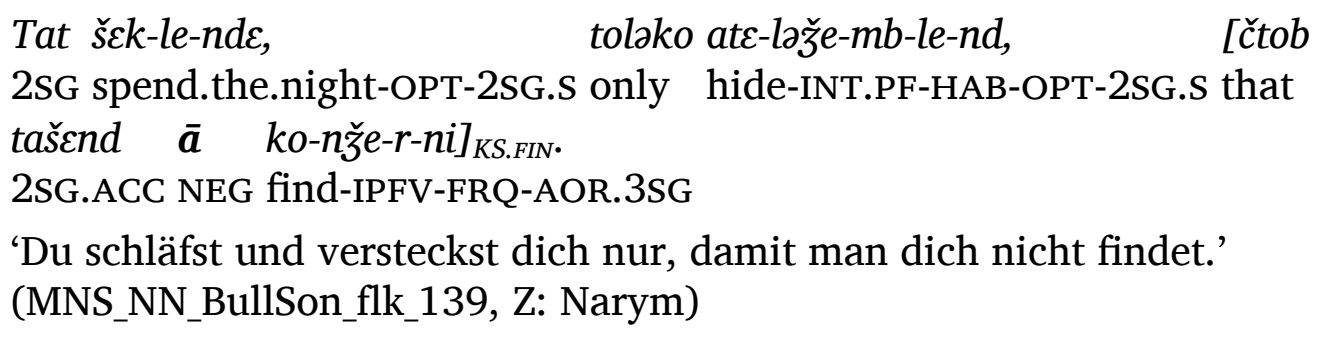

\section{Negierter Kausalsatz}

Kausalsätze, die den Grund für einen Sachverhalt näher beschreiben, finden sich im Korpus lediglich als affirmative Variante mit negiertem Matrixsatz. Negierte Kausalsätze sind ebenso wenig belegt wie nordselkupische Beispiele. In beiden Beispielen (266a) und (266b) erscheint der Kausalsatz jeweils nachgestellt. In (266a) ist das Subjekt al'č’’kat - 'Großmutter' lexikalisch overt ausgedrückt, in (266b) ist es kovert.

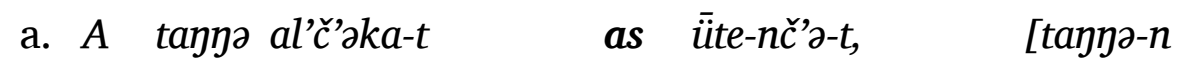
but down grandmother-3SG NEG let.go-IPFV-3SG.o down-ADV

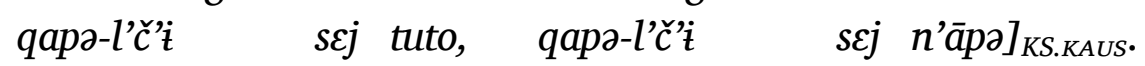
be.fat-PTCP.PRS eye crucian be.fat-PTCP.PRS eye duck

'Aber hinunter lässt ihn seine Großmutter nicht, (weil) unten schieläugige Karauschen und schieläugige Enten sind.'

(NN_1879_Itja_flk_2, S: Chaja)

b. Tita man qwasso-n ollo-m assa tuali-r-š-te-nda-ndi, now 1SG iron-GEN head-ACC NEG steal-FRQ-US-DRV-INFER-2SG.S [šinda am-na- $\eta]_{\text {KS.KAUS }}$. 2SG.ACC eat-AOR-3SG.S

'Jetzt kannst du meinen Schaberkopf nicht stehlen, (weil) ich dich aufgegessen habe.'

(SVG_1964_ItekaPineweldju_flk_42, S: Upper Ket') 


\section{Negierter Konditionalsatz}

Konditionalsätze formulieren Bedingungen, unter denen die Handlung im Matrixsatz erst stattfinden kann. Die Beispiele (267a) und (267d) zeigen einen mit der Negationspartikel ašša negierten Konditionalsatz. Er ist dem Matrixsatz vorangestellt. In den Beispielen (267b) und (267c) ist der Konditionalsatz zusätzlich mit Hilfe der aus dem Russischen stammenden Konjunktion $b \dot{i}$ - 'wenn' gebildet und geht dem Matrixsatz ebenfalls voraus. Allerdings enthält dort nur der Matrixsatz eine Negation.

(267) a. [Ašša qan-na-ntì] $]_{K S . к O N D,}$ to muntik šimin am-ta na lōsi. NEG go.away-AOR-2sG.S that all 1PL.ACC eat-FUT.3SG this devil

'Wenn du nicht gehst, wird dieser Teufel uns alle fressen.'

(BEP_1977_Icha_flk_22, N: Upper Taz)

b. [Mat tanu-p-ni-m bi் $]_{K S . K O N D}, \check{s} \bar{s} w \quad \overline{\boldsymbol{a}}$

1SG know-HAB-AOR-1SG.O if ashes NEG

kotta-l-b-ni-m bi.

throw-INCH-HAB-AOR-1SG.O if

'Wenn ich das gewusst hätte, hätte ich die Asche nicht weggeworfen.' (MNS_NN_BullSon_flk_78, Z: Narym)

c. [Man bi tunu-ne-w] $]_{K S . K O N D}$, qusa-kan $=n e j$ as me-ne-w

$1 \mathrm{SG}$ if know-AOR-1SG.O when-LOC $=\mathrm{EMPH}$ NEG give-AOR-1SG.O

onen qiban'aza-w.

himself boy-NOM/ACC.1SG

'Wenn ich das gewusst hätte, hätte ich mein Kind nie weggegeben.' (PVD_1966_BoyDevil_flk_22, S: Chaja)

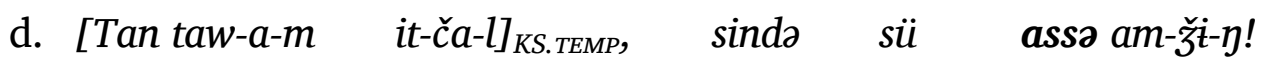

2SG this-EP-ACC drink-FUT-2sG.O 2sG.ACC dragon NEG eat-FUT-3sG.S

'Wenn du dies trinkst, wird der Drache dich nicht fressen.'

(AGS_1968_FairytaleSnake_flk_88, S: Middle Ket')

\section{Negierter Konsekutivsatz}

Konsekutivsätze benennen eine Folge des im Matrixsatz genannten Sachverhaltes. Im Korpus kommen in allen Dialektgruppen negierte Konsekutivsätze in Satzgefüigen vor, in denen der Matrixsatz affirmativ ist, wie in den Beispielsätzen (267a) - (267c). Anders als im affirmativen Beispiel (248) aus Kapitel 5 sind negierte Konsekutivsätze uneingeleitet. Eine zusätzliche Markierung im Matrixsatz mit Hilfe der Adverben namiššak - 'so viel', nidin - 'solch' und našaj - 'so viel' findet jedoch ebenfalls statt. In den Konsekutivsatz in (267c) ist darüber hinaus noch ein Finalsatz mit einer infiniten Konstruktion eingebettet. 
a. Mcrkì namǐššak $\varepsilon s \dot{i}-m p a$

$[m \bar{t} t-t \dot{t}$

nōni ponä

ašša wind thus.much become-PST.REP.3SG tent-OBL.2SG out outwards NEG tan-nEnta] $]_{\text {KS.KONSEQ }}$. go.out-INFER.FUT.3SG

'Es gab so einen Wind, dass er nicht aus seinem Zelt hinausgegangen ist.' (BVP_1973_East_flk_4, N: Taz)

b. Man nidi-n me-lle-be, [na mene-r-til kup tekka 1SG such-ADV do-OPT-1SG.O this hunt-FRQ-PTCP.PRS person NEG aga to-lla $]_{\text {KS.KONSEQ }}$. 2SG.DAT come-OPT.3SG

'Ich werde es so machen, dass dieser Jäger nicht zu dir kommt.' (ChDN_1983_HerosDaughter_flk_40, Z: Vasjugan)

c. Täb-i-nan naša-j qomde, [tab-i-nani mādə-m nāgur-i-jy 3SG-EP-ADE so.many-ADJZ money 3SG-EP-ADE house-ACC three-EP-ADV qoja-ľ̌i-ku ass mide-n̋̌i-t $]_{K S . K O N S E Q}$. surround-INT.PF-INF NEG achieve-FUT-3SG.O

'Er hat so viel Geld, dass er nicht schafft, sein Haus dreimal hineinzutun.' (PMP_1961_Fairytale_flk_197, S: Middle Ob)

Die Beispiele (269a) und (269b) zeigen ebenfalls negierte Konsekutivsätze. Allerdings ist ersterer mit der translativischen Form des Demonstrativpronomen na - 'dieser' eingeleitet, (269b) zeigt einen uneingeleiteten Konsekutivsatz. Der Matrixsatz in (269b) enthält analog zu den Beispielen (268a) - (268c) oben als zusätzliche Markierungdas Adverb $n ı k$ - 'so'. Im Unterschied zu den obigen Beispielen sind die Prädikate in (269a) und (269b) mit der Negationspartikel čä̈gk negiert, die sich aus dem negativen Existenzialverb čä $\bar{k} k i$ entwickelt hat (vgl. hierzu auch die Ausführungen von Wagner-Nagy (2011: 209f.)).

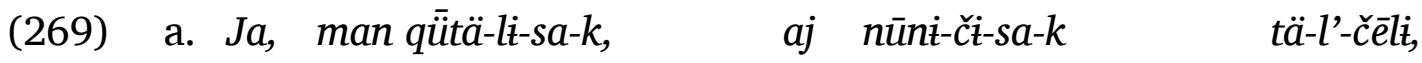
now 1SG be.ill-RES-PST-3SG.S and be.tired-TR-PST-3SG.S this-ADV-day [na-tqa čä̈jki mē-ptä-m n'äji-m] $]_{\text {NEG.KONSEQ }}$ tom-ni-t mači- $l$ this-TRL NEG do-ACT-1SG bread-ACC say-AOR-3SG.O taiga-ADJZ lōsi. devil

'Nun, ich bin müde und krank gewesen heute, sodass ich kein Brot gemacht habe, sagte der Waldgeist.'

(SNS_1966_Markincha_flk_176, N: Taz)

b. Lōsi epsi-r-i-mpi-lä nı qati-nni-t, [Markin'ča-m čääjki devil hurry-FRQ-EP-HAB-CVB so row-AOR-3SG.O Markincha-ACC NEG qo-ntit-r-ä-tti] $]_{N E G . K O N Z}$. sight-IPFV-FRQ-ACT-3SG

'Der Bär ruderte so schnell, dass er Markincha nicht bemerkte.' (SNS_1966_Markincha_flk_45, N: Taz) 


\section{Negierter Temporalsatz}

Temporalsätze beschreiben eigenständige Handlungen und ordnen sie der Handlung des Matrixsatzes zeitlich zu: vorzeitig, gleichzeitig oder nachzeitig. In Beispiel (270) findet die Handlung des Konstituentensatzes gleichzeitig mit der Handlung des Matrixsatzes statt. Der Matrixsatz ist mit der Negationspartikel assi negiert. Zentral- oder nordselkupische Beispiele sind im Korpus nicht belegt.

(270) [Māt-qandi qwen-gu ēzu-n-dì $]_{K S . T E M P}$ watta-m-di assi house-LOC.3SG go.away-INF hanker.for-AOR-3SG.O road-ACC-3SG NEG qo-kku-wi-t.

find-ITER-AOR-3SG.O

'Als sie nach Hause gehen wollte, fand sie ihren Weg nicht.' (KMS_1966_TwoSisters_flk_10, S: Middle Ket')

\subsubsection{Negierter Relativsatz}

Relativsätze als Konstituentensätze sind in den Matrixsatz eingebettet und fungieren als syntaktischer Modifikator einer NP des Matrixsatzes. Im Selkupischen kommen formal komplexe Relativsätze äußerst selten vor, typischweise fungieren Partizipien als Modifikatoren von Nominalphrasen (vgl. Kapitel 4). Beispiel (271) zeigt einen negierten Relativsatz. Er ist mit dem Interrogativpronomen qaj - 'was' eingeleitet und relativiert das pronominale direkte Objekt nawgam - 'dieses.ACC'. Der Relativsatz geht dem Matrixsatz voraus.

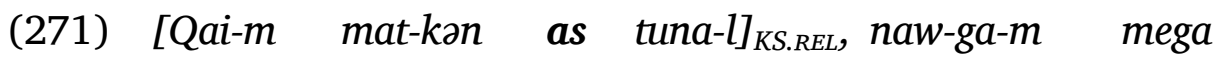
what-ACC house-LOC NEG know-2SG.O this-DIM-ACC 1SG.DAT

t'apt-et!

promise-IMP.2SG.O

'Was du zu Hause nicht kennst, das versprich mir!' (PVD_1966_BoyDevil_flk_13, S: Chaja)

Zusammenfassend lässt sich festhalten, dass das Selkupische zum Ausdruck der Standardnegation eine Negationspartikel in den Varianten ašša (nordselkupisch), aya, $\bar{a}$ (zentralselkupisch) und ass(i), aza, a (Südselkupisch) verwendet. Es unterscheidet sich damit von den nordsamojedischen Sprachen, die traditionellerweise über ein negatives Hilfsverb verfügen (Wagner-Nagy (ebd.: 317)). Auch im Selkupischen gibt es ein negatives Hilfsverb in der Form tača / čed'a, čeža / ted'a - 'nicht können', es wird jedoch außer in nordselkupischen Präteritalumgebungen nicht in der Standardnegation verwendet. Es ist in den Beispielen (264a) - (264d) und (253) gezeigt.

Existenzialsätze werden, wie in den anderen samojedischen Sprachen auch, mit einem negativen Existenzialverb ( č $\left.\bar{a} \eta k \dot{z} / \check{c} a \eta g(w) a, t^{\prime} a \eta g(w) a / t^{\prime} \ddot{a} \eta g(w) a\right)$ negiert (Beispielsätze (204a) - (204f)). Die zentralselkupischen Dialekte - und nur sie - haben die russische Negationspartikel netu als negatives Existenzialverb reinterpretiert und verwenden es ebenfalls zur Negation von Existenzialsätzen ((206a) - (206c)). Auch 
Lokativsätze (Beispiele (207a) - 207c) ) und possessive Konstruktionen werden mit dem negativen Existenzialverb negiert. Erstere lassen sich von Existenzialsätzen lediglich durch die Wortstellung unterscheiden, In Lokativsätzen geht das Thema der Lokation voraus ( $\mathrm{TH}+\mathrm{LOC}+\mathrm{COP}$ ), in Existenzial- und Possessivsätzen ist es umgekehrt. In possessiven Konstruktionen kann der Possessor unterschiedlich markiert werden: In allen Dialektgruppen kann der Possessor unmarkiert, also im Nominativ, erscheinen. Außerdem ist eine Lokativmarkierung möglich, wobei sich Nord- und Nicht-Norddialekte voneinander unterscheiden. Erstere verwenden eine Postpositionalkonstruktion (Beispiel (208c)), letztere eine morphologische Markierung (Beispiele (209a) - (209d)). Nur im Selkupischen hat sich zusätzlich in allen Dialektgruppen aus der Form der 3. Person Singular des negativen Existenzialverbs eine weitere Negationspartikel entwickelt, die im Nordselkupischen teilweise auch zum Ausdruck der Standardnegation verwendet wird. Imperative Konstruktionen werden mit einer speziellen Negationspartikel (ikk / $\dot{t} g \dot{\partial}, \ddot{o} g / \dot{i} k(\dot{t}), \partial g(\partial)$ ) ausgedrückt, die sich von der Standardnegationspartikel unterscheidet.

Innerhalb der komplexen Sätze ist auffällig, dass ausschließlich finite Teilsätze negiert werden. Infinite Konstitutentensätze, die eine konverbale Form oder einen Infinitiv enthalten, erscheinen stets in affirmativer Form (Beispiele (259a) - (259c) sowie (260a) - (260d)). Im Rahmen der vorliegenden Arbeit fehlt der Raum, um dieses Phänomen detaillierter zu betrachten. Tabelle 20 fasst die unterschiedlichen Negationsstrukturen im Selkupischen noch einmal zusammen.

Tab. 20: Negationsstrukturen im Selkupischen

\begin{tabular}{|c|c|c|c|}
\hline Dialekt & Standardnegation & Neg.Imperativ & Neg.Existenz \\
\hline Nord & 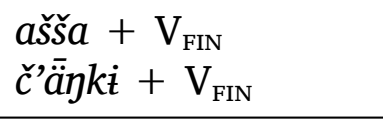 & $i k \dot{t}+\mathrm{V}_{\mathrm{FIN}}$ & 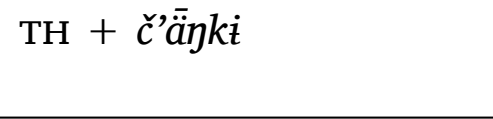 \\
\hline Zentral & $a \gamma a, \bar{a}+\mathrm{V}_{\mathrm{FIN}}$ & $\dot{t g} \dot{g}, \ddot{o} g+\mathrm{V}_{\mathrm{FIN}}$ & $\begin{array}{l}\mathrm{TH}+\check{c} a \eta g(w) a, t^{\prime} a \eta g(w) a \\
\mathrm{TH}+n^{\prime} e t u\end{array}$ \\
\hline Süd & $\operatorname{ass}(\dot{i}), a z a, a+\mathrm{V}_{\mathrm{FIN}}$ & $i k(i), \partial g(\partial)+V_{\mathrm{FIN}}$ & $\mathrm{TH}+t^{\prime} \ddot{a} \eta g(w) a$ \\
\hline
\end{tabular}

\section{Zusammenfassung}

Insgesamt kann zu komplexen Sätzen festgehalten werden, dass sowohl die Parataxe als auch die Hypotaxe ursprünglich asyndetisch ausgedrückt wurden, mittels der bloßen Nebeneinanderstellung von Teilsätzen sowie infiniten Verbformen, wie Konverben und Infinitiven. Diese Eigenschaft teilt das Selkupische mit anderen uralischen Sprachen. Die Juxtaposition formt additive Sachverhalte, wenn sie Teilsätze mit gleichwertigem syntaktischen Status verbindet (Beispiele (219a) und (219b)) und unterschiedliche adverbiale Sachyerhalte, wenn die Teilsätze syntaktisch nicht gleichwertig sind (Beispiele (233a) - (233g)). Es wurde die Schwierigkeit aufgezeigt, die 
eine klassische (europäische) Definition von Teilsätzen (dass sie notwendigerweise über eine finite Verbform und ein explizites Subjekt verfügen) bei der Betrachtung komplexer Sätze aufwirft, die mit Hilfe infiniter Verbformen gebildet werden. Typischerweise sind diese Formen von impliziten Subjekten gekennzeichnet, auch wenn explizite Subjekte vorkommen können (Beispiel (217)). Mit der Annahme, ein Teilsatz liege dann vor, wenn er ein Prädikat enthält, formen Konverben und Infinitive konverbale bzw. infinite Teilsätze. Mit Hilfe des Infinitivs werden ausschließlich finale Adverbialsätze geformt (Beispielsätze (235a) - (235e)). Der Status von Konverben im Selkupischen ist noch nicht abschließend erforscht. Konverben können allein keinen vollständigen Satz bilden, sie sind also nicht koordinierend (not dependent + not embedded) im Sinne einer europäischen Sprachbetrachtung (vgl. hierzu auch Haspelmath (1995: 3 ff.)). Dennoch werden Konverben verwendet, um eine Sequenz aufeinanderfolgender Satzteile auszudrücken, eine Funktion, die auch als ,.clause-chaining“ (Haspelmath (ebd.: 7)) oder „medial-final chaining“ (Longacre (2007: 375)) bezeichnet wird. Zusätzlich werden Konverben als Teil komplexer Prädikate verwendet, die im Exkurs-Kapitel 6 beschrieben sind. Da im Rahmen der vorliegenden Arbeit der Status selkupischer Konverben nicht abschließend geklärt werden kann, erfolgte ihre Beschreibung eher pragmatisch orientiert innerhalb der koordinierten (Beispiele (220a) - (220f)) bzw. subordinierten Sätze (Beispiele (234a) - (234f)).

Weiterhin konnte gezeigt werden, dass unter russischem Einfluss syndetische komplexe Sätze gebildet werden. Sie können sowohl koordinierende als auch subordinierende Sachverhalte ausdrücken und werden typischerweise durch entlehnte Konjunktionen eingeleitet. Insbesondere für die adverbialen Satztypen Konzessivsatz (Beispiel (248)), Konsekutivsatz (Beispiel (249)) und Temporalsatz (Beispiele (250a) und (250b) sind nur einzelne Beispiele belegt. Eine Besonderheit bilden Kausalsätze, die nur im Südselkupischen belegt sind, und in denen nur der Matrixsatz mit Hilfe des Adverbs nano - ‘dann' bzw. der Translativform von na - 'dieser' gekennzeichnet ist (Beispiele (240a) - (240c)). Der eigentliche Konstituentensatz wird ohne Konjunktion angeschlossen.

Komplementsätze werden mittels Juxtaposition (Beispiele (233d) - (233f) undKonjunktionen gebildet. Dabei können selkupische Interrogativpronomen wie in (236a) - (236d) oder eine aus dem Russischen entlehnte Konjunktion wie in den Beispielen (238a) - (238a) verwendet werden.

Abschließend wurden in Abschnitt 5.2.4 Relativsätze beschrieben, die als formal komplexe Sätze hauptsächlich nach russischem Muster gebildet werden. Dabei fungieren originär russische Interrogativa als Relativpronomen (Beispiele (252a) - (252c)). Ursprünglich werden attributive Relativsätze im Selkupischen nur auf Phrasenebene mittels Partizipien realisiert. Dennoch kommen auch komplexe Relativsätze vor, die als Konstituentensatz mit „Lücke“ an den Matrixsatz angeschlossen werden (Beispiele (251a) - (251c)). Wie alle Modifikatoren, erscheinen sie typischerweise unmittelbar vor dem Kopfnomen. Tabelle 21 fasst die Strukturen in komplexen Sätzen zusammen. 


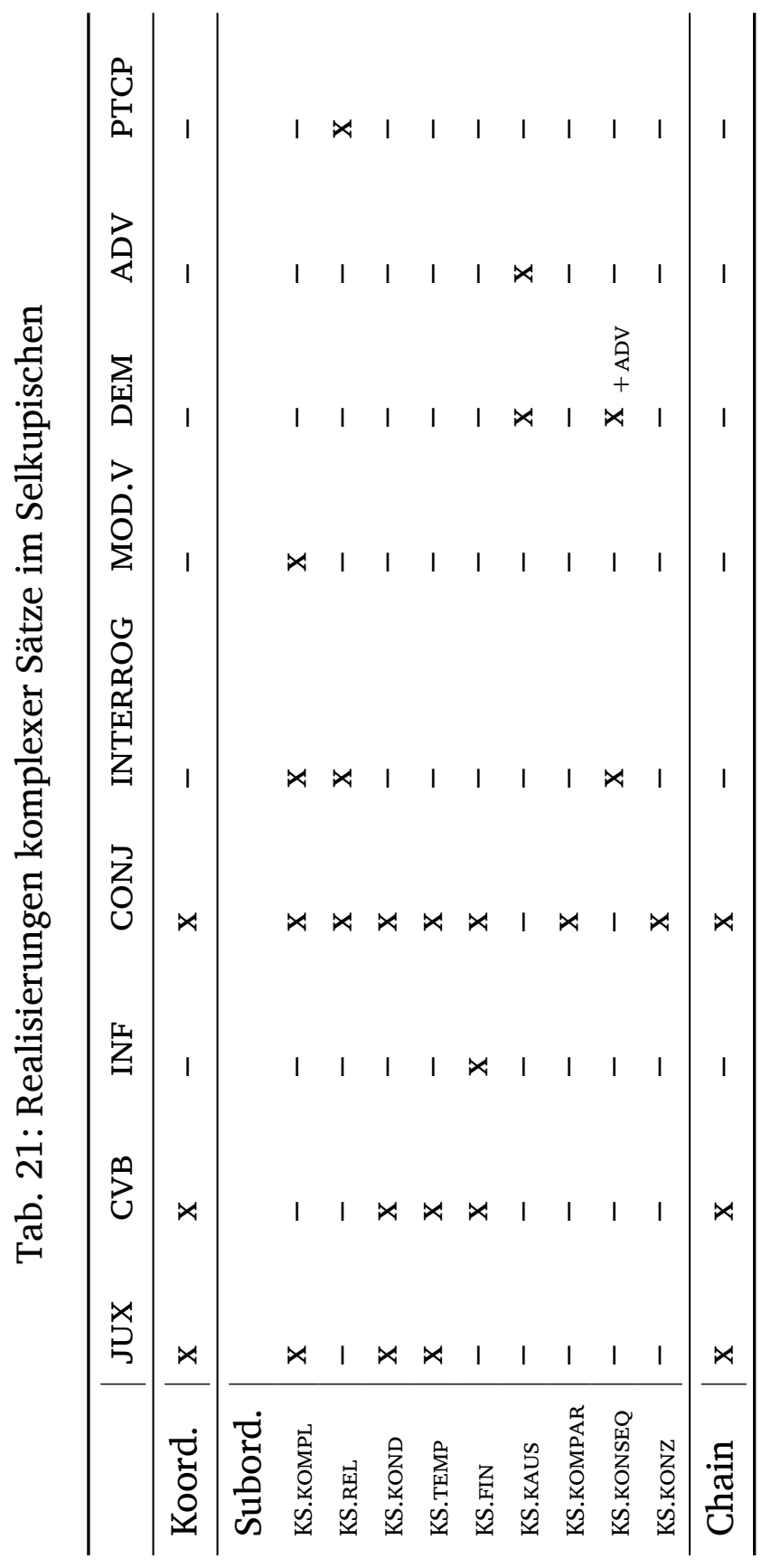




\section{Exkurs Konverbkonstruktionen}

In den vorangegangenen Kapiteln sind unterschiedliche Funktionen von Konverben aufgezeigt worden. Dazu gehören syntaktische Funktionen, wie die eines Adverbials in Kapitel 2.8 oder ihre Verwendung in komplexen Sätzen zum Ausdruck subordinierter oder koordinierter Sachverhalte, wie in Kapitel 5. Weiterhin gehören auch Konverbkonstruktionen als formales Mittel zur Grammatikalisierung aspektueller Hilfsverben dazu, die hier als komplexe konverbale Prädikate bezeichnet werden.

Dieses Exkurs-Kapitel soll den Raum bieten, um einerseits diese für uralische Sprachen untypische Konstruktion genauer betrachten zu können, und andererseits Konverbkonstruktionen auch aus dem Nordselkupischen zu berücksichtigen. Es wird sich zeigen, dass das Nordselkupische (ebenso wie die zentralselkupischen Dialekte) nicht nur erheblich weniger Konverbkonstruktionen verwendet als das Südselkupische, sondern außerdem mit komplexen Prädikaten, die eine Form von olam - 'beginnen' enthalten, über eine Ausdrucksmöglichkeit verfügt, die sich weder im Zentral- noch im Südselkupischen findet.

\subsection{Einleitung}

Anders als bei den syntaktischen Funktionen, die eine Konstruktion stets vom Konverb ausgehend betrachtet, steht bei der Beschreibung von Konverben als Teil komplexer Prädikate der finite Teil der Konstruktion im Fokus. Nach vorläufigen Analysen lassen sich derartige Konverbkonstruktionen im Selkupischen in vier Gruppen unterteilen: In Gruppe A (Abschnitt 6.4.1) ist der finite Teil ein Phasenverb. Mit Konverbkonstruktionen der Gruppe B (Abschnitt 6.4.2) werden komplexe Bewegungen ausgedrückt. Die dritte Gruppe (Abschnitt 6.4.3) bilden Konstruktionen, die aus zwei semantisch identischen oder ähnlichen Lexemen bestehen und mit deren Hilfe eine Handlung hervorgehoben wird. Die letzte Gruppe (Abschnitt 6.4.4) schließlich bilden konverbale Konstruktionen, deren finiter Teil als Hilfsverb fungiert, um verschiedene aspektuelle Lesarten und Aktionsarten auszudrücken.

Das Forschungsbild zeigt sich sowohl bezüglich des Begriffs Konverb im Allgemeinen als auch selkupischer Konverben und -konstruktionen im Speziellen uneinheitlich. Der Terminus „Converbum“, von Ramstedt (1903: 55) in der Altaistik eingeführt, diente dort der Bezeichnung (Khalkha-)mongolischer Verbalformen, die er als „halb verbale, halb nominale formen des verbums" beschreibt. Er assoziierte dabei den Begriff historisch und morphologisch mit verbalen Nomen, da „die khalkhassischen konverba auf ganz demselben Niveau wie diejenigen kasusformen der nominalen ver- 
balformen [stehen], die als bestimmungen zum hauptverbum angewendet werden können ..." (Ramstedt 1903: 55). Der Terminus wurde in einen breiteren typologischen Kontext übernommen und wird synonym mit Begriffen wie „gerund“ (Latein, Englisch), ,adverbiales Partizip“ (Slawisch),., deepričastie“ (Russisch) und „gérondif“ (Französisch) verwendet. (vgl. u.a. Tikkanen 2001, Haspelmath 1995). Die Betrachtung in der Fachliteratur konzentriert sich dabei entweder mehr auf den verbalen oder den nominalen Charakter der Verbform (siehe Ramstedt oben). Im Kontext der europäischen Sprachbetrachtung verweist der Terminus Konverb auf „a nonfinite verb form whose main function is to mark adverbial subordination. Another way of putting it is that converbs are verbal adverbs, just like participles are verbal adjectives." (ebd.: 3). Fokussiert wird auf den verbalen Charakter des Converbums, da Konverben in europäischen Sprachen typischerweise eine rein adverbiale, modifizierende Funktion haben, wie in Beispiel (272).

(272) Non ave-ndo la chiave, non potè aprire.

[not have-CONV the key] not could open

'Not having the key, she couldn't open.'

(Haspelmath 1999: 1, Italienisch)

Nedjalkovs Definition ist breiter gefasst: Ein Konverb ist „a verb form which depends syntactically on another verb form, but is not its syntactic actant, i.e., does not realize its semantic valencies ..." (Nedjalkov 1995: 97). Derartige Definitionen, die Konverben vor allem singulär betrachten, reduzieren sie auf ein grammatisches Mittel zur Wiedergabe syntaktischer Verbindungen. Obwohl Nedjealkov Konverben vorrangig syntaktisch betrachtet, steht seine Annahme, „a canonical (i.e. noncombined) converb [...] cannot occupy the positions [...] of a clausal actant (i.e. it cannot depend on verbs such as begin, order, etc.) ... (ebd.: 97) dem in verschiedenen Sprachen Zentralsibirien (vgl. hierzu Anderson (2004b: 74ff.) belegten) Vorkommen von konverbalen komplexen Prädikaten entgegen. Aus diesem Grund wird hier die Betrachtung von Konverben auf die gesamte Konstruktion (Konverb und finite Verbform) erweitert, um derartige Phänomene adäquat untersuchen und beschreiben zu können.

Johanson beschreibt unter diesem Aspekt Konverbkonstruktionen im Türkischen und spricht von „converb segments (CS) to be a nonfinite unit which is constructurally subordinated to a base segment (BS)“ (Johanson 1995: 313). Das von ihm dargestellte Modell zur Beschreibung türkischer Konverbkonstruktionen ist in vier Stufen (levels) unterteilt: Auf der ersten Stufe sind BS und CS volle Prädikate mit jeweils eigenen (ersten) Aktanten (Subjekt). Auf der zweiten Stufe referieren BS und CS auf dasselbe Subjekt, BS formt ein zweites Prädikat. In der dritten Stufe sind BS und CS ein Prädikat und formen eine Verbalphrase, in der BS als Hilfsverb erscheint. Merkmale dieser Stufe sind die starke semantische Verschmelzung, da nur noch ein Ereignis wiedergegeben wird sowie die Tendenz zur Lexikalisierung. Auf der vierten Stufe des Grammatikalisierungsprozesses sind CS und BS derart verschmolzen, dass BS lediglich noch als grammatischer Marker für CS fungiert. Auf dieser Stufe sind die Konverben in der Regel aspektuell. 


\subsection{Konverbverbindungen in uralischen Sprachen}

Für verschiedene uralische Sprachen werden Konverben bzw. Konverbkonstruktionen beschrieben. Hierzu gehören Chantisch (Fil'chenko 2010: 470), Udmurtisch (Pischlöger 2001, Winkler 2011: 117 ff), Marisch (Bradley 2016), Nenzisch (Nikolaeva 2014: 110, 372ff. Pischlöger 2001: 47, Décsy 1966: 50-51), Enzisch (Sieg1 2013: 325) und Kamassisch (Klumpp 2002). Auf den eher nominalen Charakter von Konverben konzentriert sich die Beschreibung für das Chantische (Fil'chenko 2010: 470). Das Konverb kann hier Kasussuffixe annehmen und auch als Nominalisierung betrachtet werden. Ähnliche Konstruktionen finden sich auch für das Nganasanische, wie beispielsweise bei Tereshchenko (1979) oder Katzschmann (2008). Für das Enzische beschreibt Siegl (2013: 325f) ein „infinitival converb“, dessen Suffix jedoch identisch mit dem des Infinitivs ist, sowie ein temporales Konverb, wie in Beispiel (273). Auch für das Nenzische wird als Hauptfunktion adverbiale Subordination angegeben (Nikolaeva 2014: 110) und so im Haspelmathschen Sinne zu den „Verbal Adverbs (Gerunds)“ gezählt.

(273)

stol ke-xun adi-š minxuda kariđa sei-gu-̌̌s

table-GEN side-LOC.SG sit-CVB at once fish.ACC.PL.3SG clean-DUR-CVB

pä.

begin.3SG

'Sitting at the table, he started to clean the fish at once.'

(Siegl (2013: 326), Waldenzisch)

Konverbkonstruktionen im Udmurtischen, Marischen und Kamassischen orientieren sich am verbalen Charakter des Konverbs und ähneln denen im Selkupischen beobachteten, wie im kamassischen Beispiel (274). Derartige konverbale Konstruktionen sind untypisch für uralische Sprachen und durch Sprachkontakt entstanden, wie z. B. Marisch-Tschuwaschisch (Bradley 2016: 269) und Kamassisch-Chakassisch (Klumpp 2002: 330ff.).
šut $\quad k u^{c} p t o ̄ l a g a$
[kuPbdolaga < kuPbdola(?) ige].
fire.3SG extinguish.IPF.PRS
extinguish-CVB be.PRS
'Sein Feuer war am Erlöschen.'
(Klumpp (2002: 203), Kamas)

In diesen Sprachen haben sich unter dem Einfluss verschiedener Turksprachen Konverbkonstruktionen zu Hilfsverbverbindungen entwickelt (zu turksprachigen Konverben in Hilfsverbverbindungen siehe auch Schönig (1984) und Anderson (2004a), Anderson (2004b). Das Selkupische befindet sich ebenfalls im turksprachigen Kontaktgebiet (wie das Kamassische). Es kann daher angenommen werden, dass der Einfluss auf selkupische Strukturen gleichermaßen relevant ist. 


\subsection{Konverbverbindungen im Selkupischen}

Die vorhandenen grammatischen Arbeiten zeigen für das Selkupische ein eher uneinheitliches Bild. Konverben bzw. Konverbkonstruktionen werden in verschiedenen grammatischen Beschreibungen erwähnt (Voevodina 1976, A. I. Kuznecova/ Chelimskij/Grushkina 1980, Helimski 1983, Helimski 1998, Cheremisina/Martynova 1991, N. G. Kuznecova 1995, Valijärvi 2008). In der Regel wird formal zwischen einfachen Konverben auf -le und komplexen Formen auf -pūle (Nordselkupisch) oder auf der verschmolzenen und grammatikalisierten Form -lewle (oder eine der Varianten, im Zentral- und Südselkupischen) unterschieden. Teilweise erfolgt eine dialektale Zuweisung von Funktionen, basierend auf den Formen (Ausdruck von Gleichzeitigkeit bzw. Ungleichzeitigkeit). Das bedeutet, Konverben werden vor allem auf der morphosyntaktischen Ebene betrachtet. Zudem stützt sich die Mehrzahl der Abhandlungen größtenteils auf Daten aus den nördlichen Dialekten des Selkupischen, wie A. I. Kuznecova/Chelimskij/Grushkina (1980) und Valijärvi (2008). In Helimski (1983) und Cheremisina/Martynova (1991) werden auch zentral- und südselkupische Konverben berücksichtigt. N. G. Kuznecova (1995) behandelt in ihrer Monographie ebenfalls südselkupische Konverbkonstruktionen. Sie konzentriert sich jedoch neben einer morphologischen Beschreibung der Konverben vor allem auf die Etymologie der Form(en). Konverben in Hilfsverbverbindungen (siehe oben) werden teilweise genannt, eine vollständige Systematik bzw. Typisierung fehlt bisher jedoch völlig.

Basierend auf den Korpusdaten können in den drei selkupischen Dialektgruppen einfache und komplexe Konverben unterschieden werden. Einfache Konverben werden mit dem Suffix -le gebildet, komplexe Konverben sind zusammengesetzt aus dem einfachen Konverb auf -le und der konverbalen Form -pūle - 'kreuzen.cvB' (siehe hierzu auch A. I. Kuznecova/Chelimskij/Grushkina (1980: 256ff.), Cheremisina/Martynova (1991: 36), N. G. Kuznecova (1995: 195f.), Valijärvi (2008: 169ff.)). Die Suffixe in den einzelnen Dialektgruppen sind z.T. verschmolzen bzw. verkürzt und treten in den Varianten -lebele (-lebe, -ple) in den südlichen Dialekten und -ble (-ple) in den zentralen Dialekten (es finden sich hier nur vereinzelte Beispiele für komplexe Konverben) auf. In den Ket'-Subdialekten des Südselkupischen werden komplexe Konverben mit dem Suffix -lewle gebildet, einem Allomorph zu -lebele.

Das Nordselkupische verwendet neben einfachen Konverben auch komplexe Formen, wobei die in den zentralen und südlichen Dialekten beobachtete Verschmelzung hier nicht stattfindet, wie in (275).

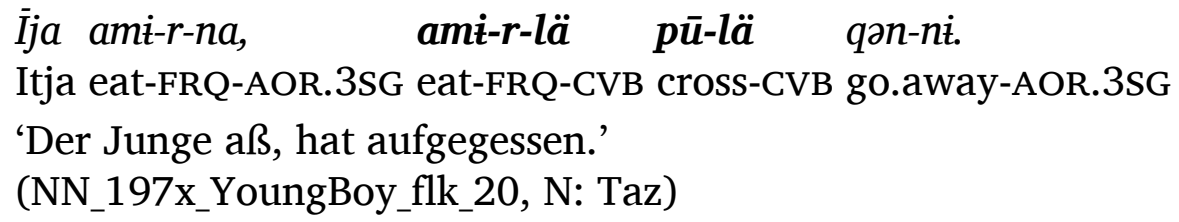

Selkupische Konverbkonstruktionen kommen in allen dialektalen Gruppen vor, jedoch mit sehr unterschiedlicher Frequenz, wie Tabelle 22 zeigt. 
Konverben können Derivationssuffixe tragen, die Kategorien Person, Numerus, Tem-

Tab. 22: Häufigkeit von Konverben in den selkupischen Dialekten

\begin{tabular}{l|ll}
\hline Dialektgruppe & Sätze mit CVB-Konstruktionen & Verhältnis zur Gesamtzahl der Sätze \\
\hline Nord & 156 & $16,1 \%$ \\
Zentral & 148 & $15,3 \%$ \\
Zentral/Süd & 117 & $12,1 \%$ \\
Süd & 547 & $56,5 \%$ \\
\hline
\end{tabular}

pus und Modus werden jedoch ausschließlich an der finiten Verbform markiert. Selkupische Konverbkonstruktionen referieren in der Regel auf dasselbe Subjekt. Eine der wenigen Ausnahmen ist in Beispiel (276) gezeigt. Das Beispiel zeigt auch, dass infinite Verbformen im Selkupischen explizite Subjekte haben können und somit einen Teilsatz formen (vgl. hierzuauch die Bemerkungen hinsichtlich der Definition des Terminus 'clause' in Kapitel 4).

(276) Tepqin qwe-lewl'e, it'e amdi-qu- $\eta$.

3DU fish-CVB Itja sit-ITER-3SG.S

'Sie gingen fischen, und Itja sitzt.'

(KKN_1971_Itja_flk_40), S: Upper Ket')

\subsection{Komplexe (konverbale) Prädikate}

Im Folgenden werden die vier Gruppen beschrieben, in die selkupische Konverkonstruktionen unterteilt werden. Die Klassifizierung hat vorläufigen Charakter, da die diesbezüglichen Analysen erst begonnen haben. Für ein adäquates Klassifizierungsschema sind weitere und detailliertere Untersuchungen notwendig. So sollte beispielsweise herausgearbeitet werden, ob und welche Phasenverben (Gruppe A) in Gruppe D eingeordnet werden können 1 . Diese Untersuchungen können jedoch im Rahmen der vorliegenden Arbeit nicht durchgeführt werden.

\subsubsection{Gruppe A}

Gruppe A beinhaltet komplexe Prädikate, die aus einem Konverb und einem der Phasenverben olda - 'beginnen', kwatta - 'beginnen', übə - 'beginnen', mančə (Zentralselkupisch) bzw. malča (Südselkupisch) - 'beenden' bestehen. Typischerweise geht das Konverb der finiten Form voraus. Vor allem in den zentralen Dialekten werden die Phasenverben auch mit dem Infinitiv anstelle eines Konverbs verbunden (siehe unten).

Im Südselkupischen Teilkorpus sind alle Phasenverben belegt. Sie werden praktisch

\footnotetext{
${ }^{1}$ Vgl. Schönig (1984: 43) für eine diesbezügliche Diskussion für das Tatarische.
} 
ausnahmslos mit Konverben verwendet. Die Beispiele (277a) bis (277c) zeigen die unterschiedlichen Lexeme von 'anfangen'. In Beispiel (278a) und (278b) ist malč 'aufhören' gepaart mit den konverbalen Formen au 'essen', einer dialektalen Variante von am bzw. kual - 'töten', einem Allomorph von qwat.

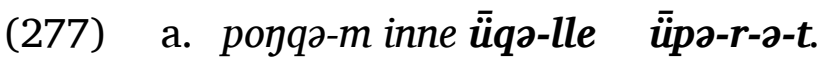

net-ACC up drag-CVB begin-FRQ-EP-3SG.O

'Er beginnt, das Netz hochzuziehen.'

(NN_1879_Itja_flk_43, S: Chaya)

b. pōne-la-n pal'd'u-l'e oldi- $\boldsymbol{\eta}$.

outside-PL-LOC.ADV go-CVB begin-3SG.S

'Er beginnt, hinauszugehen.'

(PVD_1961_FarmAssault_flk_145, S: Middle Ob)

c. Lōz-la tö-mba-t i kozir-č-le kwatta-lža-t.

devil-PL come-PST.REP-3SG.O and card-TR-CVB begin-INT.PF-3SG.O

'Teufel kamen und begannen Karten zu spielen.'

(SEV_1981_SisterBrother_flk_38, S: Middle Ob)

(278) a. aur-le malčā-ga.

eat-CVB stop-3DU.S

'Sie hörten auf zu essen.'

(PVD_1961_FarmAssault_flk_146, S: Upper Ob)

b. Nagur-mželi čelea-gand kual-le malče-d.

three-ORD day-LOC.3SG kill-CVB stop-3SG.O

'Am dritten Tag hörte er auf zu töten.'

(NN_1855_Hero1_flk_167, Z/S: Tym/Middle Ob)

Es gibt eine Handvoll südselkupischer Beispiele, in denen anstelle eines Konverbs der Infinitiv verwendet wird, wie in (279), meist sind es Übersetzungen aus dem Russischen. In diesen Fällen erscheint der Infinitiv konsequent nach der finiten Form.

(279) Tü-mbadi qū-la i-ya-tta utto-yinda qūja-la-m come-PTCP.PST person-PL tale-AOR-3PL hand-LOC.3SG landing.net-PL-ACC i oldi-mba-tto manži-mbi-gu. and begin-PST.REP-3PL look-HAB-INF

'Die Gäste (wrtl.: angekommenen Menschen) nahmen Kescher in ihre Hände und begannen zu schauen.' 
(KMS_1967_Hunt_trans_49, S: Middle Ket')

Für die gemischte Dialektgruppe Zentral/Süd sind Beispiele mit kuatta - 'beginnen' und ein Beispiel mit malče - 'beenden' belegt. Analog zu den südlichen Dialekten

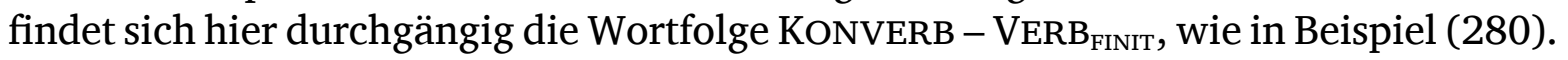

Kuače-p pače-lle üba-r-a-p.

city-ACC cut.down-CVB begin-FRQ-EP-1sG.O

'Ich begann, die Stadt niederzuhauen.'

(NN_1855_Hero4_song_83, Zentral/Süd)

Im zentralselkupischen Subkorpus kommen Phasenverben insgesamt nur selten vor. Von diesen erscheinen lediglich manč - 'beenden' in (281a) und qwatti? - 'beginnen' in (282a) in Konverbkonstruktionen.

a. Tabə ǚ̌'u-ku-k,

üza-le man-rr-ēe-špa-t,

3SG work-ITER-3SG.S work-CVB finish-FRQ-EP-IPFV-3SG.O

uboršicā-n tēntra: pōne-t mēkka qwā-gu,

female.cleaner-DAT speak.3SG outside-LOC.ADV 1SG.DAT go.away-INF

ku-n'ibet pad'ī mozno?

where-any just one.can

'Er beendet seine Arbeit und sagt zu seiner Frau: Kann ich jetzt rausgehen?'

(SDP_1964_FairytaleBlackZar_flk_376, C: Narym)

b. Mete-ร̌e-la kartoš-ka-p paqqi-le qwati-l-de.

reach-IPFV-CVB potatoe-DIM-ACC dig-CVB begin-INCH-3SG.O

'Er kommt dort, beginnt nach Kartoffeln zu graben.'

(SDP_1964_FairytaleBlackZar_flk_835, C: Narym)

In allen anderen Beispielen werden Phasenverben mit dem Infinitiv verwendet. Das Verb 'beginnen' erscheint in den Lexemen lakki, titi und qwatti. In den meisten Fällen geht die finite Form dem Infinitiv voraus, wie in (282b). In allen Beispielen aus dem Vasyugan-Dialekt und imn einzigen Beispiel aus dem Tym-Dialekt ist die umgekehrte Wortfolge zu beobachten, wie in (282a) und (282c). Detailliertere Aussagen lassen sich aufgrund der wenigen Beispiele nicht treffen.
a. tab ku-d-e-n
kwen-gu
laka-tte-ku-k,
id'e
3SG person-PL-EP-DAT/ALL go.away-INF begin-IPFV-ITER-3SG.s Itja
ku-d-e-n aya üdi-ld'e, pokko-m-de
person-PL-EP-DAT/ALL NEG send-INT.PF.3SG net-ACC-3SG
ora-l-lad'e.
hold-INCH-INT.PF.3SG

\footnotetext{
${ }^{2}$ Bis auf ein Beispiel erscheint das Phasenverb nur mit Konverben.
} 
'Beginnt er, zu Menschen zu gehen, lässt Itja ihn nicht gehen, hält ihn mit dem Netz.'

(ChDN_1983_ItjasTown_flk_16, C: Vasyugan)

b. Nä-l-gup ando-ute paht-r-a aj

woman-ADJZ-PERSON boat-PROL jump-FRQ-AOR.3SG and

titti-ži-mba paža-le-špe-gu.

begin-IPFV-PST.REP.3SG chop-INCH-IPFV-INF

'Die Frau sprang aus dem Boot und begann, (Bäume) zu fällen.'

(KIA_2014_KatManPuch_trans_40, C: Narym)

c. Kušā-göt tat lèma-p mē-gu kuöttāa-lža-l, to when-LOC.ADV 2SG board-ACC do-INF begin-INT.PF-2SG.O then tärbe-š manžö-gu kaj-l-žön $\bar{e}-m a$.

think-IMP.2SG.S look-INF what-ADJZ-? be-COND.3SG

'Wenn du beginnst, ein Brett zu machen, dann bedenke, von welchem Stamm es ist.'

(KAO_1912_SmartPeople_trans_8, C: Tym)

Im nordselkupischen Subkorpus gibt es nur ein Beispiel( $\bar{u} k \dot{i}-$ 'beginnen'), in dem ein Phasenverb mit einem Konverb gepaart ist, wie in (283a). Das einzige andere Phasenverb, das im Korpus belegt ist, ist olam - 'beginnen'. Es erscheint immer in infiniten Konstruktionen, in denen der Infinitiv des lexikalischen Verbs mit der finiten Form von olam verschmolzen ist, wie in (283b). Diese Konstruktion weist Ähnlichkeiten zum kamassischen Beispiel (283c) auf, in dem die konverbale Form mit dem Auxiliar verschmolzen ist als Ergebnis eines Grammatikalisierungsprozesses.

a. Qum-i-t ami-r-lä ūki-taltō-tit. person-EP-PL eat-FRQ-CVB begin-DRV-3PL

'Die Menschen begannen zu essen.'

(BVP_1973_East_flk_42, N: Taz)

b. Nini ili-q-olam-n̄̄-tit.

then live-INF-begin-AOR-3PL

'Dann begann sie zu leben.'

(BIV_1941_KonMytyke_flk_34, N: Taz)

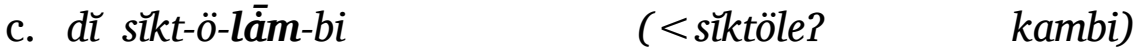

he strangle-INTR-ASPECT-PAST (< strangle.INTR.CV go.away.PAST)

'Er erhängte sich selbst.'

(Klumpp 2002: 286) 


\subsubsection{Gruppe B}

Konverbale Konstruktionen, die eine komplexe Bewegung ausdrücken, gehören zur Gruppe B. Diese Konstruktionen bestehen aus zwei Bewegungsverben, von denen das Konverb die Art der Bewegung (gehen, laufen, fliegen, ...) wiedergibt, die finite Form (hinausgehen, weggehen, ...) den Pfad 3 .

Die meisten Beispiele dieser Gruppe, wie in (284), stammen aus den südselkupischen Dialekten. Tabelle 23 zeigt sämtliche Kombinationen von Bewegungsverben in den südlichen Dialekten.

Tab. 23: Kombinationen von Bewegungsverben in Konverbkonstruktionen im Südselkupischen

\begin{tabular}{|c|c|}
\hline Konverb & Finites Verb \\
\hline $\begin{array}{l}\text { tö - 'come', tayə - 'crawl', qwan - 'go } \\
\text { away', wässa, timbi - 'fly', } \overline{\ddot{u}} \text { ta - 'send, } \\
\text { let go', s'ila - 'sharp', } \bar{u} \text { - 'swim' }\end{array}$ & medi - 'reach' \\
\hline $\begin{array}{l}\text { timbi - 'fly', qwaja - 'go', tudi - 'reach', } \\
\text { kur - 'run', ü̈t - 'send, let go' }\end{array}$ & tö - 'come' \\
\hline $\begin{array}{l}\text { qwetz - 'catch', tȫ - 'come', kur - 'run', } \\
\text { tita - 'walk' }\end{array}$ & $\check{c} \bar{a} \breve{z} \partial$ - 'go' \\
\hline tanyə - 'crawl', mendi - 'pass through' & $q o-$ - 'go' \\
\hline $\begin{array}{l}\text { pü - 'cross', čanže - 'go out', laqqa - } \\
\text { 'move, work', tita - 'walk' }\end{array}$ & qwon - 'go away' \\
\hline čači - 'leave' & $k w e d i-$ 'leave' \\
\hline sape - 'spring out' & čaže - 'run' \\
\hline tangi - 'throw out', $\bar{u}$ - 'swim' & $\overline{u ̈ t z ~-~ ' s e n d, ~ l e t ~ g o ' ~}$ \\
\hline
\end{tabular}

(284) Aba-t kur-l'e t'ü-a-n, k'ed'egu-m t'abə-t. old.sister-3SG run-CVB come-AOR-3SG.s basket-ACC catch-3SG.O 'Ihre ältere Schwester kam angerannt, und nimmt den Korb.' (PMP_1961_ForestWoman_flk_123, S: Middle Ob)

In sämtlichen Heldenliedern der gemischten Dialektgruppe finden sich Beispiele aus der Gruppe B., wie in (285a) und (285b). Die analysierten Kombinationen unterscheiden sich von denen im Südselkupischen und sind in Tabelle 24 aufgelistet.

\footnotetext{
${ }^{3}$ Konverbale Konstruktionen, in denen die Handlung auf das semantische Feld der 'Jagd' referiert, machen die Hälfte aller Konverbverbindungen aus. Sie werden nicht zur Gruppe B gezählt. Ob diese Konstruktionen als Finalsätze betrachtet werden können (vgl. Kapitel 5) kann noch nicht eindeutig festgestellt werden.
} 
Tab. 24: Kombinationen von Bewegungsverben in Konverbkonstruktionen im Zentral/Südselkupischen

\begin{tabular}{|c|c|}
\hline Konverb & Finites Verb \\
\hline tiö- 'come' & medi - 'reach' \\
\hline kur - 'run' & tö - 'come' \\
\hline tö - 'come', qwan - 'go away' & alče - 'fall' \\
\hline para - 'return', tö - 'come' & čaร̌e - 'go' \\
\hline čanže - 'go out', para - 'return' & qwən - 'go away' \\
\hline qoja - 'surround' & čanže - 'go out' \\
\hline
\end{tabular}

(285)

a. ku-la-m pone čanže-le kuan-na-det.

person-PL-1SG outside go.out-3PL go.away-AOR-3PL

'Meine Leute gingen hinaus.'

(NN_1855_Hero3_song_309-310, Zentral/Süd)

b. nat.kund keba kuene-k kur-a-lbele tö-k.

there.long small brother.in.law-DIM run-EP-CVB come-3sG.S

'Währenddessen kam der kleine Schwiegersohn angerannt.'

(NN_1855_Hero2_song_377-378, Zentral/Süd)

Im Zentralselkupischen ist die Verwendung von Konverbkonstruktionep mit Bewegungsverben nur bei einem Sprecher des Narym-Dialektes belegt. Tabelle 25 gibt eine Übersicht über die belegten Kombinationen. Sämtliche Beispiele sind Übersetzungen aus dem Russischen. Die konverbale Form geht der finiten Form immer voraus, wie in (286).

Tab. 25: Kombinationen von Bewegungsverben in Konverbkonstruktionen im Zentralselkupischen

\begin{tabular}{ll}
\hline Konverb & Finites Verb \\
\hline čaga - 'hurry' & čaร̌e - 'go' \\
hokko - 'crawl' & kcntṫ - 'sneak up' \\
čaga - 'hurry' & tö - 'come' \\
\hline
\end{tabular}

(286) Tab čageti-mb-le tö-ha limb mogo-git tu-la-he.

3SG hurry-HAB-CVB come-AOR.3SG eagl back-LOC wing-PL-INSTR

'Er lief schnell zum geflügelten Adler.'

(KIA_2014_GiantZobel_trans_77, C: Narym) 
Es gibt nur wenige Beispiele der Gruppe B im nordselkupischen Subkorpus. Sämtliche Paare enthalten $q \partial n$ - 'weggehen' als finite Verbform, die Konverben variieren (siehe Tabelle (26)). In Beispiel (287) ist die Art der Bewegung 'springen', ausgedrückt durch das Konverb pakti, der Pfad wird durch die finite Form von qan - 'weggehen' ausgedrückt.

Tab. 26: Kombinationen von Bewegungsverben in Konverbkonstruktionen im Nordselkupischen

\begin{tabular}{ll}
\hline Konverb & Finites Verb \\
\hline$p \bar{u}$ - 'cross', poqqitti - 'settle.net', tōpirì & qan - 'go away' \\
- 'pick.berry', pakti - 'jump' & \\
\hline
\end{tabular}

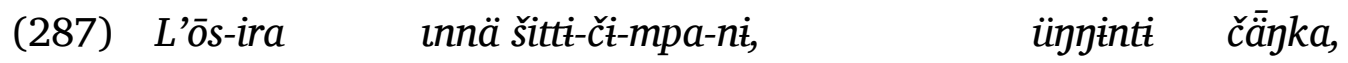
devil-old.man up wake.up-DRV-HAB-AOR.3SG wolverine NEG.EX.3sG

pakti-lä qən-pa-ni.

jump-CVB go.away-HAB-AOR.3SG

'Der teuflische Alte wacht auf, das Vielfraß ist nicht da, es hüpfte davon.' (MIV_1977_Icha_flk_52, N: Taz)

\subsubsection{Gruppe C}

Zur Gruppe C werden Konverbkonstruktionen gezählt, in denen die Handlung hervorgehoben bzw. verstärkt wird. In den relevanten Beispielen liegen das Konverb und die finite Verbform semantisch nah beieinander. Eine ähnliche Konstruktion gibt es beispielsweise in tuwinischen Konstruktionen, in denen das Konverb selbst verdoppelt wird, um Kontinuität oder Durativität auszudrücken (vgl. Aydemir (2009: 65f.)). Erste Analysen zeigen Beispiele von fünf verschiedenen Sprechern des südselkupischen Ket'-Dialektes wie in (288a) - (288c). Darüberhinaus gibt es zwei Beispiele aus dem südselkupischen Middle Ob-Dialekt wie in (288d) und zwei weitere Beispiele aus dem nordselkupischen Taz-Dialekt wie in (288e). Für das Zentralselkupische lassen sich dagegen im Korpus keine Beispiele nachweisen 4 . Auch im Kamassischen, der nächstverwandten Sprache zum Selkupischen, sind derartige Konstruktionen bisher nicht belegt. Hinsichtlich der verwendeten Verben unterscheiden sich die Konstruktionen wie folgt: Konverb und finites Verb können ein und dasselbe Lexem verwenden, wie püta ${ }^{5}$ - 'ertasten' in (288a) oder semantisch ähnlliche Lexeme, wie kuča - 'schlafen

\footnotetext{
${ }^{4}$ Aufgrund der eher geringen Anzahl konverbaler Konstruktionen im Zentralselkupischen überhaupt lässt sich aus dem Nichtvorhandensein von Beispielen aus dieser Gruppe keine zuverlässige Aussage darüber treffen, ob solche Konstruktionen in den zentralselkupischen Dialekten generell vorkommen.

${ }^{5}$ Die finite Verbform ist mit dem Inchoativasuffix -lē aktionsartderiviert, die Bedeutung des Lexems wird damit jedoch lediglich modifiziert.
} 
gehen' und qonda - 'schlafen' in (288b), tšuka - 'Wurm' und tä - 'verrotten' in (288c), tita - 'schreiten und t'ive - 'schreiten' in (288d) oder qätti - 'schlagen' und sil'l'alti 'niederschlagen' in (288e).

(288) a. A tita uzo püta-le-lle pü̈ta-lēenda-t but now already track.down-INCH-CVB track.down-INCH-IPFV-3SG.O säp-qa-nde- $\eta$. pocket-DIM-OBL.3SG-LOC.ADV

'Und er tastest nun schon (lange) in seiner Tasche.' (SVG_1964_IitekaPineweldju_flk_46, S: Upper Ket')

b. Awu-r-na-di, $\bar{t} r-a-d i \quad$ kuča-l'e qonda-di. eat-FRQ-AOR-3DU.O drink-EP-3DU.o go.to.sleep-CVB sleep-3DU.O 'Sie essen, trinken und legen sich schlafen.' (KMS_1966_TwoSisters_flk_42, S: Middle Ket')

c. Na sūrum wät't'e tšuka-r-l'e tä-mba, tšuka-m this wild.animal meat worm-VBLZ-CVB rot-PST.REP.3SG worm-ACC üt-man tādo-r-i-t. water-PROL bring-FRQ-EP-3SG.O

'Dieses Tierfleisch ist verwurmt, es bringt die Würmer durch das Wasser.' (KNI_1964_ItjaCapeOfWorms_flk_69-70, S: Upper Ket')

d. kut'e-t mat t'iye-ľse-špa-y t'itta-r-lä tan naj man-nani where-ADV 1SG walk-TR-IPFV-1SG.S walk-FRQ-CVB 2SG also 1SG-ADE

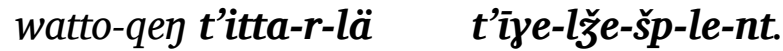

road-LOC walk-FRQ-CVB walk-TR-IPFV-OPT-2sG.S 'Wo ich gehe, dort sollst du auf meinem Weg gehen.' (TFF_1967_ItjaAldigaPoenegesse_flk_6, S: Middle Ob)

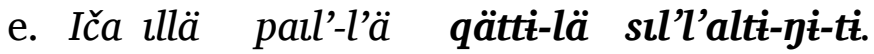
Itja down jump-CVB beat-CVB break.down-AOR-3SG.O 'Itja kletterte hinunter und erschlug ihn.' (AVA_1973_Ichakicha2_flk_176, N: Taz)

\subsubsection{Gruppe D}

Die vierte Gruppe komplexer konverbaler Prädikate bildet Auxiliarverbindungen. Das finite Verb in diesen Konstruktionen ist im Zuge eines Grammatikalisierungsprozesses der semantischen Reduktion unterschiedlich weit als Auxiliar grammatikalisiert. Es verfügt nicht mehr über seine volle semantische Bedeutung, sondern ist vielmehr 
semantisch reduziert, „somewhat semantically bleached“, wie Bradley (2016: 45) es ausdrückt. Es kann so als Auxiliar fungieren, wobei ein Teil der ursprünglichen Bedeutung erhalten sein und Einfluß auf die Bedeutung der Konstruktion als Ganzes nehmen kann. Klumpp verwendet hier den Begriff 'semantically reducible' anstelle von 'semantically reduced' (Klumpp 2005a: 401). So stehen die Bedeutungen des Auxiliars 'sitzen' und des Konverbs 'weinen' in (289) nicht im Konflikt miteinander.

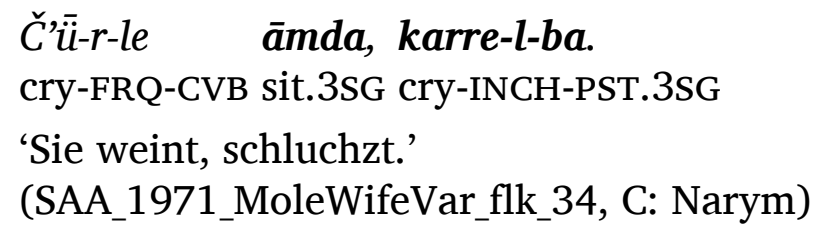

Das Auxiliar in derartigen Verbindungen ist vorrangig Träger verschiedener grammatischer Informationen wie Tempus, Modus, Person und Numerus. Das Konverb hingegen drückt (primär) die lexikalische Bedeutung des komplexen Prädikats aus und kann mit unterschiedlichen Derivationen modifiziert werden. Konverbale Auxiliarverbindungen werden verwendet, um unterschiedliche Aspekt- oder Aktionsartlesarten auszudrücken. Aufgrund der strukturellen Ambiguität sind die Lesarten der Auxiliarkonstruktionen oftmals nicht eindeutig. In Beispiel (289) oben könnte das Konverb auch adverbial übersetzt werden: 'Sie sitzt weinend'. Teilweise sind auch subordinierende oder koordinierende Lesarten möglich. Zur Disambiguisierung wären Beispiele hilfreich, in denen die wörtliche Übersetzung von Konverb und finiter Verbform keinen rechten Sinn ergibt, wie das von Klumpp (2002: 136) angeführte kamassische Beispiel in 2906. Im selkupischen Korpus fehlen derartige Beispiele. Erschwerend kommt im selkupischen Korpus hinzu, dass der Text als Ganzes (sinngemäß) übersetzt ist. Damit wird das Selkupische oftmals nur unzureichend wiedergeben.

(290) Amno-la i’bym.

sit-CVB lie.AOR.1SG

'Ich sitze.'

(Kamassisch)

Die Klassifikation der Verben in der Gruppe D orientiert sich an Schönigs Klassifizierung der tatarischen Äquivalente (Schönig 1984), die wiederum auf der von Johanson (1971) entwickelten Unterscheidung transformativ|nontransformativ beruht. Die finiten Verben der Gruppe D sind in Anlehnung an Schönig (1984: 34) unterteilt in initialtransformative, finaltransformative und nontransformative Verben. Erste Analysen zeigen neun Verben, die als Auxiliar verwendet werden (siehe Tabelle 27).

\footnotetext{
${ }^{6}$ Eine wörtliche Übersetzung 'Ich sitze liegend' ergibt keinen wirklichen Sinn, zudem lautet die originale russische Übersetzung ja sizhu - 'ich sitze', und unterstützt damit die Disambiguisierung.
} 
Tab. 27: Auxiliare in konverbalen Konstruktionen

\begin{tabular}{|c|c|c|}
\hline $\begin{array}{l}\text { transformativ } \\
\text { initial }\end{array}$ & final & nontransformative \\
\hline $\begin{array}{l}\text { qali - 'bleiben' } \\
\text { qwande - 'wegtragen' } \\
q(w) \partial n \text { - 'weggehen' }\end{array}$ & $\begin{array}{l}\text { tāda - 'bringen' } \\
\text { omdə - 'setzen' }\end{array}$ & $\begin{array}{l}\check{c} \bar{a} \bar{z} \partial \text { - 'gehen' } \\
\bar{a} \sim \text { omda - 'sitzen' } \\
\text { ippí - 'liegen' } \\
n \dot{t} \text { - 'stehen' }\end{array}$ \\
\hline
\end{tabular}

Die klassifizierten Hilfsverben interagieren mit Aktionalphrasen ('Sachverhalt' bei Sasse (1991)), die ebenfalls unterteilt sind in spezifizierung bei Schönig). Tabelle 28 zeigt die Bedeutungsmodifikationen, die bei der Interaktion der Hilfsverben mit den Aktionalphrasen entstehen. Sie ist an Klumpp (2002) angelehnt.

Tab. 28: Interaktion von Auxiliaren und Aktionalphrasen

\begin{tabular}{llll}
\hline Auxiliar & initialtransformativ & $\begin{array}{c}\text { Aktionalphrase } \\
\text { finaltransformativ }\end{array}$ & nontransformativ \\
\hline nontransformativ & stativ oder seriell & stativ oder seriell & stativ oder seriell \\
\hline initialtransformativ & initial & 'postfinal' & initial \\
\hline finaltransformativ & initial & final & final \\
\hline
\end{tabular}

\subsubsection{Initialtransformative Auxiliare}

Als initialtransformative Auxiliare können die Verben qali - 'bleiben', qwande - 'wegtragen' und $q(w) \partial n$ - 'weggehen' fungieren. In Kombination mit nontransformativen bzw. initialtransformativen Aktionalphrasen betonen sie die linke Grenze und formen inchoative Bedeutungen. Zusammen mit finaltransformativen Aktionalphrasen werden 'postfinale' Bedeutungen (postdesinentiell bei Klumpp (2002: 166)) geformt.

$$
\text { qali - 'bleiben' }
$$

Das Verb qali - 'bleiben' markiert die linke Grenze eines Ereignisses, sowohl mit nontransformativen als auch mit initialtransformativen Auxiliaren. In Kombination mit finaltransformativen Ereignissen liegt der Fokus außerhalb des Sachverhaltes, allerdings sind für diese Kombination keine Korpusbeispiele belegt. Ebenso fehlen Beispiele aus den zentralen Dialekte. In den (291a) und (291b) aus dem Südselkupischen sowie in (291c) aus der gemischten Dialektgruppe wird qali in stativen Situationen

\footnotetext{
${ }^{7}$ Die finaltransformativen Aktionalphrasen sind bei Schönig weiter unterteilt in präliminar- final-
} transformativ und momentan-finaltransformativ. 
mit den Konverben amdi - 'sitzen' (291a), meše - 'krank sein' (291b) und ora - 'halten' (291c) verwendet. Das Konverb in (291b) ist zusätzlich mit dem Inchoativsuffix -lamarkiert. Im nordselkupischen Beispiel (291d) indiziert qali eine dynamische Situation mit dem Konverb nitz - 'zerreißen'.

(291) a. Pēk'k'e taw pil'eka-yon ämdi-l'e qali-y. hazel.grouse this side-LOC sit-CVB stay-3SG.S

'Das Haselhuhn bleibt auf dieser Seite sitzen.' (KMS_1966_MouseGray_flk_28, S: Middle Ket')

b. Kalabokka meše-la-č-lä qāla i ōl-tar-gālin.

Kalabokka ill-INCH-TR-CVB stay.3SG and head-hair-CAR

'Kalabokka wurde krank und blieb ohne Haare.'

(TFF_1967_KolobokkaPoenegessa_flk_28, S: Middle Ob)

c. Čuečo-nd katte-d, čemn'a-nd ol-nda laga-p ora-lbele earth-ILL beat-3SG.o brother-OBL.3sG head-ILL heap-ACC hold-CVB

kal-tte-d.

stay-DRV-3SG.O

'Auf die Erde er (ihn) schlägt, vom Kopf des Bruders ein Stück er ergriff (und es festhielt).'

(NN_1855_Hero4_song_41-43, Zentral/Süd)

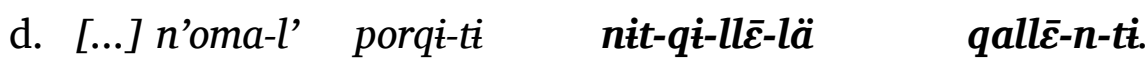

[...] hare-ADJZ clothing-3SG tear-DRV-INCH-CVB stay-AOR-3SG.O

'[...] zerriss sein Hasenpelzmantel (und blieb in ihren Händen).'

(KMP_1971_NyomalPorky_flk_35, N: Taz)

\section{qwand $\varepsilon$ - 'wegtragen'}

Im Korpus finden sich zwei Beispiele, in denen qwande - 'wegtragen' als Auxiliar verwendet wird. Das initialtransformative Verb zeigt ebenfalls eine Situationsveränderung an und erzeugt mit nontransformativen (Beispiel (292a)) und initialtransformativen (Beispiel (292b)) Aktionalphrasen eine inchoative Lesart.

\section{a. T'äptä t'äk köt-l'e qwända-ku-t.}

tale fast say-CVB carry.away-ITER-3SG.O

'Das Märchen ist schnell erzählt.'

(AGS_1968_FairytaleSnake_flk_47, S: Middle Ket')

b. Mat ora-nna-p, pone čače-le kuanda-p, po-t

1SG hold-AOR-1SG.O outwards throw-CVB carry.away-1SG.O tree-GEN

par-o-nd čaps-a-p.

top-EP-ILL spear-EP-1SG.O 
'Ich nahm sie, warf sie hinaus auf die Straße, tat sie auf einen Baum.' (NN_1855_Hero2_song_405-407, Zentral/Süd)

q(w)ən - 'weggehen'

$q(w) \partial n$ - 'weggehen' ist das dritte initaltransformative Auxiliar. Im Korpus sind zwei Beispiele aus dem südselkupischen Ob-Dialekt belegt. In (293a) ist die Aktionalphrase finaltransformativ, das Auxiliar konnin - 'weggehen.3SG' betont hier die rechte Grenze des Ereignisses und erzeugt darüber hinaus eine 'postfinale' Lesart. In (293b) erscheint qwat - 'weggehen', als eine Variante von qwan, mit dem finaltransformativen Konverb sällä - 'schärfen'. Der Fokus liegt hier ebenfalls auf dem Verlassen der Situation, die durch das Konverb beschrieben wird.

a. Sada qiba n'ē qiba.n'e t'ari-y oj pazan'e $i$ two small daughter small.daughter say-3SG.S INTERJ Baba-Yaga and qow-l'a kon-ni-y. drown-CVB go.away-AOR-3sG.s

'Zwei kleine Töchter, kleine Töchter, ooh, sagt Baba-Jaga und ertrank.' (PMP_1961_ForestWoman_flk_168, S: Middle Ob)

b. I-wə-t kibizo pawə-m i säl-d-sä pō-qən take-AOR-3SG.O boy knife-ACC and grinder-3SG-LOC stone-LOC säl-lä qwat-ti-t. sharpen-CVB go.away-DRV-3SG.O

'Er nimmt ein kleines Messer und schärft es auf einem Schleifstein.' (TFF_1967_KolobokkaPoenegessa_flk_10, S: Middle Ob)

\subsubsection{Finaltransformative Auxiliare}

Die Verben tāda - 'bringen' und omda - 'setzen' fungieren als finaltransformative Auxiliare. Mit initialtransformativen Aktionalphrasen formen sie inchoative Bedeutungen, mit finaltransformativen und nontransformativen Sachverhalten hingegen resultative.

tādə - 'bringen'

Das finaltransformative Verb tādə - 'bringen' dient als Resultativmarker und zeigt an, dass ein Ereignis beendet ist. Die Handlung kann auf einen Rezipienten ausgerichtet sein, und als Resultat befindet sich das Objekt beim Rezipienten, wie in (294c). tādo ist in Texten aus dem zentralen, gemischten und südlichen Dialekten belegt. Im Ket'Dialekt ist die Form lediglich in aus dem Russischen übersetzten Texten belegt, wie (294a). In südselkupischen Texten erscheint tāda immer mit dem Derivationssuffix $-r$ - 8 , wie in (294a) bis (294c). In zentralen Texten und der gemischten Dialektgruppe

\footnotetext{
${ }^{8}$ Bisher konnte die genaue Wirkung des Suffixes noch nicht geklärt werden. Es ist unwahrscheinlich,
} 
sind auch Formen ohne Derivationssuffix belegt, wie in (295a) und (295b).
a. Täp qai-m-da=kka
tutto-l'l'e tāda-r-ì-t,
sä̈tšim

3SG what-ACC-INDEF $=$ EMPH chew-CVB carry-FRQ-EP-3SG.O severely

kwäi-no.

breath-AOR.3SG

'Er kaut irgendetwas (zu Ende), atmet schwer.'

(KMS_1968_Burbot_trans_137, S: Middle Ket')

b. A nan ugon mazim qwa-l'e tādə-r-ə-za-nt.

and so earlier 1SG.ACC beat-CVB carry-FRQ-EP-PST-2SG-S

'Aber früher hast du mich geschlagen.'

(PMP_1961_BodylessHead_flk_111, S: Middle Ob)

c. İd'ä-n ald'aga-t nā kätə-m kuddar $\bar{e}-s \quad q w \bar{e} s ' e$ Itja-GEN grandmother-3SG this gut-ACC how be-PST.3SG backward pel-le tādo-r-o-t.

put-CVB carry-FRQ-EP-3SG.O

'Itjas Großmutter steckte den Magen so, wie er vorher war.'

(NN_1913_Itja_flk_71, S: Chaya)

(295) a. Ör-le tatta-ut nedel kund.

drink-CVB bring-1PL week long

'Wir feierten (wrtl.: tranken) die ganze Woche.'

(SAI_1984_StoryAboutLifeLong_nar_27, C: Narym)

b. oker madur waše-lebele tado-ga-mba, ende-m

one hero get.up-CVB bring-ITER-PST.REP.3sG bow-ACC

oran-ne-d.

hold-AOR-3SG.O

'Ein Held stand auf, nahm den Bogen.'

(NN_1855_Hero2_song_138-141, Zentral/Süd)

omdə - 'setzen'

Das zweite finaltransformative Auxiliar ist omdə - 'setzen'. Beispiele können in allen Dialektgruppen belegt werden. Wie in anderen samojedischen Sprachen gibt es im Selkupischen ein zweites Lexem $\bar{a} \sim o m d a$, um das atelische Konzept 'sitzen' $\vartheta$ auszu-

dass es sich hier um das Frequentativsuffix handelt. Der transformative Charakter spricht dagegen. Denkbar wäre seine Funktion als ein Transitivsuffix oder ein Verblassen der ursprünglichen frequentativen Bedeutung bei einer erstarrten Form.

${ }^{9}$ Alatalo 2004: 10-11: amdi-, sit' and omdə-, sit down'. 
drücken (detailliertere Beschreibung unten). Aufgrund des Fehlens einer Schriftsprache sind beide Lexeme oft nur schwer zu unterscheiden. Wie tāda oben markiert auch omda die rechte Grenze ejnes Ereignisses und formt resultative Lesarten, wie in den Beispielen (296a) - (296c) unten.

a. Šar-uat-pa-ut, sombla kuel salt saru madur pür-k talk-DRV-PST.REP-1PL seventy.five hero round-ADV koja-lže-le omna-mba-dat. surround-TR-CVB sit.down-PST.REP-3PL

'Wir redeten und fünfundsiebzig Helden bildeten einen Kreis.' (NN_1855_Hero3_song_262-266, Zentral/Süd)

b. Unč'a-r-l'e ōmta-yi.

look.for.lice-FRQ-CVB sit-3DU.S

'Sie suchten nach Läusen.'

(PMP_1961_ForestWoman_flk_59, S: Middle Ob)

c. Nūni-či-lä omni-mpa-ni.

be.tired-DRV-CVB sit-HAB-AOR.3SG

'Er wurde müde.'

(MIV_1977_Icha_flk_38, N: Taz)

\subsubsection{Nontransformative Auxiliare}

Als nontransformative Auxiliare sind im Korpus die Verben čāža - 'gehen', ippi 'liegen', āmda - 'sitzen' und $n \dot{t}$ - 'stehen' belegt. Unabhängig von der Aktionalphrase, in der sie verwendet werden, formen nontransformative Auxiliare stative oder serielle Lesarten.

čā̋̌̀ - 'gehen'

Wie alle anderen nontransformativen Auxiliare auch, liegt die Funktion von čăž - 'gehen' in der Aufhebung der Grenzen eines Ereignisses. Anders als der stative Charakter von $\bar{a} \sim$ omda - 'sitzen' in (299a), (299b) und (301) unten, hat čāžว stets dynamischen Charakter. Beispiele finden sich nur im Südselkupischen (Ob- und Ket'-Dialekte), wie in (297a) und (297b), wo čažว mit orim - 'wachsen' verbunden ist, hier in der Variante oruw.

(297) a. Qiban'aza wārkə-n, wārkə-n, wārzə-n oruw-lēe čāze-n. boy play-3sG.s play-3sG.s big-ADV grow.up-CVB go-3sG.s

'Der Junge spielte und spielte, er ist groß geworden.' (PVD_1966_BoyDevil_flk_26, S: Upper Ob) 


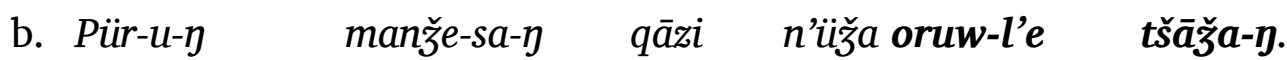
round-EP-ADV look-PST-3SG.s Russian grass grow.up-CVB go-3sG.S

'Ich sah überall Russisches Gras wachsen.'

(PES_1964_Lgov_trans_53, S: Lower Ket')

\section{ippi - 'liegen'}

Wie čă ̌̌ว fungiert ippi - 'liegen' als nontransformatives Auxiliar und formt stative oder serielle Sachverhalte. Anders als im Kamassischen erscheint ippi ausschließlich nontransformativ. Die initialtransformative Bedeutung 'legen' wird mit einem anderen Lexem (pen) ausgedrückt. Es ist gegenwärtig (noch) nicht möglich, die genaue Funktion von ippi zu bestimmen, da es im Korpus lediglich ein Beispiel gibt, in dem es in einer Konverbkonstruktion verwendet wird. Beispiel (298a) stammt aus dem südselkupischen Ob-Dialekt. ippi wird dort in der (unmarkierten) Präsensform verwendet und drückt das Andauern der Handlung - krank sein - aus. Einerseits liegen kranke Menschen häufig, so dass die Bedeutung von ippi hier auch die Vollverbbdeutung sein könnte. Andererseits ist die Konverbkonstruktion im tatarischen Beispiel (298b) von Schönig (1984: 235) als Auxiliarverbindung beschrieben, was den Hilfsverbcharakter von ippi unterstützt. Hier sind weitere Forschungen nötig, um ein eindeutige Antworten geben zu können.
a. $\overline{\tilde{U}} d \partial-\min \quad t \overline{\ddot{o}}-w a-q \quad \bar{a} d^{\prime} a-t \quad i \quad \bar{a} w o-t \quad a$ evening-PROL come-AOR-3DU.S father-3SG and mother-3SG but
Kalabokka meše-la-č-lä eppa $i$ ol-tar-gālin.
Kalabokka be.ill-INCH-TR-CVB lie.3SG and head-hair-CAR
'Am Abend kommen sein Vater und seine Mutter und Kalabokka ist krank und ohne Haare.'
(TFF_1967_KolobokkaPoenegessa_flk_29, S: Middle Ob)

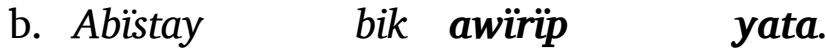
woman.master very become.ill.cVB lie
'Die alte Frau ist sehr krank.'
(Tatarisch)

ā omdə - 'sitzen'

Das nontransformative Verb $\bar{a} \sim \supset m d \partial$ - 'sitzen' ist das am häufigsten verwendete Auxiliar im Korpus und in allen Dialektgruppen belegt. Es ist das atelische Pendant zum finaltransformativen omdə - 'setzen' oben. Das Auxiliar $\bar{a} \sim$ omdə markiert immer durative, frequentative, iterative oder habituative Aspektlesarten bzw. Aktionsarten. Ähnlich zum Kamassischen kann auch im Selkupischen beobachtet werden, dass $\bar{a} \sim$ omdə in Situationen verwendet wird, deren Tätigkeiten tatsächlich im Sitzen ausgeführt werden können, wie die Konstruktionen mit čū $\breve{c}$ 'ü - 'weinen' in (299a) und (299b) und mirse - 'hobeln' in (299c). 
a. ami-tit čū-rí-lä jmta-ní.

mother-3SG cry-FRQ-CVB sit-AOR.3SG

'Seine Mutter weint.'

(MIV_1977_Icha_flk_64, N: Taz)

b. Č'̆̈̈-r-le āmda, karre-l-ba.

cry-FRQ-CVB sit.3SG cry-INCH-PST.REP.3SG

'Sie weint, schluchzt.'

(SAA_1971_MoleWifeVar_flk_34, C: Narym)

c. İra qwända-ga-j mïrse-tši-lle ämda.

old.man old-DIM-ADJZ planes-TR-CVB sit.3SG

'Der alte Mann hobelt.'

(KNI_1964_ItjaCapeOfWorms_flk_23, S: Upper Ket')

nì - 'stehen'

Wie $\bar{a} \sim э m d a$ - 'sitzen' und ippi - 'liegen' fungiert das Verb ni - 'stehen' als nontransformatives Auxilar und formt stets eine stative Bedeutung. Die inchoativ-stativen Bedeutungen 'aufstehen', 'stellen' oder 'aufhören' werden mit Hilfe des Inchoativsuffixes -le- (nile 'aufstehen') oder anderen Lexemen, wie utə, malč - 'aufhören' oder azi, wazə - 'aufstehen' ausgedrückt10. Im Korpus gibt es nur ein Belegbeispiel für eine Verwendung von $n \dot{t}$ als Auxiliar. Beispiel (300) aus dem zentralselkupischen Vasjugan-Dialekt zeigt $n \dot{t}$ mit einer Aoristmarkierung und mit dem Konverb pudi 'atmen' verbunden.

(300) Na korg akoška-t ko-ndi to-ga, nindi pudi-le this bear window-GEN side-ILL here come-AOR.3SG breath-CVB

ni-pa.

stand-AOR.3SG

'Dieser Bär kommt zum Fenster, atmet dort.'

(ChDN_1983_BearCameIntoVillage_nar_7, C: Vasyugan)

Im Subkorpus der gemischten Dialektgruppe sind einige Beispiele belegt, in denen das initialtransformative Verb nile - 'aufstehen' verwendet wird. In allen Beispielen ist es mit dem intransitiven Verb tō - 'kommen' verbunden, wie in (301). In Anlehnung an Klumpp (2005a: 405)[19]klumpp:2005b hat nile hier eine mirative Funktion, die im Türkischen -(V)p turur ein formales Äquivalent hat (vgl. Johanson (2000: 72ff.)).

(301) Nagur-mžali katkaek tö-le nile-že-k.

three-ORD 1SG.DAT/ALL come-CVB stand.up-DRV-3SG.S

'Der dritte (Held) kam plötzlich zu mir.'

(NN_1855_Hero4_song_131-133, Zentral/Süd)

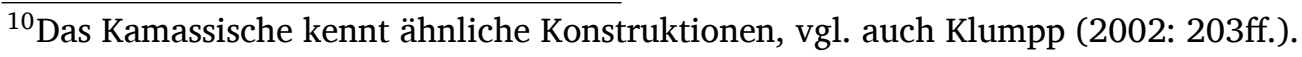




\subsection{Zusammenfassung}

Ausgehend von der Beobachtung einer großen Diskrepanz hinsichtlich der Anzahl von konverbalen Konstruktionen des Typs Konverb + Finite Verbform im zugundeliegenden Textkorpus, sollte dieses Exkurs-Kapitel vorrangig das unübersichtliche Feld derartiger Konstruktionen ordnen und eine erste Klassifizierung vornehmen. Hierzu wurden die finiten (lexikalischen) Verbformen in konverbalen Konstruktionen in vier Gruppen A-D unterteilt. Die Klassifizierung der Verben in Gruppe D orientiert sich an der von Schönig (1984) bzw. Klumpp (2002) vorgenommenen Einteilung analoger Konstruktionen für das Tatarische bzw. das Kamassische.

Es lässt sich beobachten, dass einige lexikalische Verben in konverbalen Konstruktionen einen Grammatikalisierungsprozeß durchlaufen (haben) und sich in dessen Zuge zu Auxiliaren entwickelt haben, vgl. auch Abbildung 5. Für die dritte Entwicklungsstufe sind keine Beispiele im Korpus belegt. Unterschiedliche Phasenverben

Lexikalisches Verb ---> Aspektuelles Auxiliar ---> Aspektuelles Suffix

Abb. 5: Grammatikalisierungsprozess in konverbalen Konstruktionen

sind Teil konverbaler Konstruktionen des Typs A (6.4.1). Ähnlich wie die transformativen Auxiliare (qali - 'bleiben', qwands - 'wegtragen', $q(w) \partial n$ - 'weggehen', tāda - 'bringen', omdə - 'setzen') drücken sie den Beginn oder das Ende einer Handlung aus. Im Gegensatz zu diesen spezifizieren sie jedoch nicht die Phasenstruktur der Konverbkonstruktion von der sie Teil sind: „Bezeichnet wird lediglich das Anfangen einer Tätigkeit, und da jede Tätigkeit, die stattfindet, auch anfängt, erklärt sich somit auch das Fehlen jeglicher kombinatorischer Restriktionen“, wie Schönig (1984: 269) es für das tatarische Phasenverb bašla 'anfangen' beschreibt.

Bisher konnte noch nicht eindeutig geklärt werden, ob und welches des Phasenverben mit der Bedeutung 'aufhören' als finaltransformatives Auxiliar wie im Marischen (Bradley 2016) und Tatarischen (Schönig 1984) verwendet werden kann.

Konverbale Konstruktionen, mit_Hilfe derer komplexe Bewegungen ausgedrückt werden, gehören zur Gruppe B (6.4.2) Sie sind in allen Dialektgruppen in den Beispielen (284) - (287) belegt. Die finite Verbform in diesen Konstruktionen beschreibt den Pfad der Handlung, das Konverb die Art der Bewegung. Trotz des „Durchschlagens“ der Vollverbbedeutung konnte bisher noch nicht eindeutig geklärt werden, ob die semantische Bedeutung bei Vertretern dieser Gruppe tatsächlich reduziert ist und ob sie an dem oben beschriebenen Grammatikalisierungsprozeß teilhaben.

Einige spezielle Konverbkonstruktionen sind der Gruppe C (6.4.3) zugeordnet. In den Beispielen (288a) - (288e) ist die Aktionalphrase hervorgehoben, indem sie aus semantisch gleichen (Beispiel (288a)) bzw. ähnlichen Lexemen (Beispiele (288b) - 
(288e)) in der konverbalen und der finiten Form besteht. Über die genaue Funktion der Gruppe lassen sich in Ermangelung von Beispielen keine klaren Aussagen treffen. Die Untersuchung weiterer Sprachdaten könnte abhelfen.

Eindeutig dem Grammatikalisierungsprozess lassen sich die finiten Verben der Gruppe D (6.4.4) zuordnen. Die finiten Verbformen in den Beispielen (289) - (301) befinden sich auf der zweiten Stufe des Grammatikalisierungsprozesses. Sie fungieren als Auxiliar und drücken unterschiedliche aspektuelle Bedeutungen und Aktionsarten aus. Schönig (1984) und Johanson (1971) folgend werden diese Verformen weiter unterteilt in intitialtransformative, finaltransformative und nontransformative Auxiliare (Tabelle 27 in 6.4.4). Auxiliare sind semantisch reduziert, jedoch bleibt ein Teil ihrer lexikalischen Bedeutung erhalten und kann in der Bedeutung der konverbalen Verbalphrase „durchschlagen“. Die Funktion der Auxiliare ist die Markierung durativer, frequentativer, iterativer oder habituativer Aktionsarten (nontransformative Verben čā̌̆o - 'gehen', ippi - 'liegen', āmda - 'sitzen' and ni - 'stehen' ) einerseits, sowie ingressiver, inchoativer (initialtransformatives Verb qali - 'bleben'), egressiver und resultativer Aktionsarten (finaltransformative Verben tādə - 'bringen', qwands 'wegtragen', $q(w) a n$ - 'weggehen') andererseits.

Aus Tabelle 29 lässt sich entnehmen, dass die meisten Auxiliare in einem der südlichen Dialekte belegt sind. Die naheliegende Schlussfolgerung, die Verwendung der Auxiliare folge einem Süd-Nord-Gefälle ist dabei lediglich eine Möglichkeit. Das Fehlen einer größeren Menge von Sprachdaten in den übrigen Dialektgruppen erschwert eine eindeutige Aussage.

Tab. 29: Dialektale Verteilung der einzelnen Auxiliare

\begin{tabular}{|c|c|c|c|c|}
\hline & Nord & Zentral & Zentral/Süd & $\overline{\text { Süd }}$ \\
\hline $\begin{array}{l}\text { initialtransformativ } \\
\text { qali - 'bleiben' } \\
\text { qwands - 'wegtragen' } \\
q(w) \partial n \text { - 'weggehen' }\end{array}$ & $\mathbf{X}$ & $\mathrm{X}$ & $\mathrm{X}$ & $\begin{array}{l}\mathrm{X} \\
\mathrm{X} \\
\mathrm{X}\end{array}$ \\
\hline $\begin{array}{l}\text { finaltransformativ } \\
\text { tāda - 'bringen' } \\
\text { omdə - 'setzen' }\end{array}$ & & $\mathbf{X}$ & $\begin{array}{l}\mathrm{X} \\
\mathrm{X}\end{array}$ & $\begin{array}{l}X \\
X\end{array}$ \\
\hline $\begin{array}{l}\text { nontransformativ } \\
\text { čāžว - 'gehen' } \\
\text { ippí - 'liegen' } \\
\bar{a} \sim \text { omdə - 'sitzen' } \\
n \dot{t} \text { - 'stehen' }\end{array}$ & $\begin{array}{l}\mathrm{X} \\
\mathrm{x}\end{array}$ & $\begin{array}{l}\mathbf{X} \\
\mathbf{X}\end{array}$ & $\begin{array}{l}\mathrm{S} \\
\mathrm{X}\end{array}$ & $\begin{array}{l}\mathrm{X} \\
\mathrm{X} \\
\mathrm{x}\end{array}$ \\
\hline
\end{tabular}

Andererseits sind die für uralische Sprachen untypischen konverbalen Konstruktionen, in denen finite Verbformen als Auxiliar verwendet werden, sowohl für das 
Kamassische (Klumpp 2002) als auch für das Marische (Bradley 2016) beschrieben. Dort haben sie sich unter dem Einfluss von Turksprachen entwickelt, Chakassisch als Kontaktsprache für das Kamassische und Chuwaschisch für das Marische. Ein analoger Einfluss auf die Entwicklung der finiten Verbformen in konverbalen Auxiliarkonstruktion ist demzufolge sehr wohl möglich. Zumal das Selkupische, insbesondere die südselkupischen Dialekte in (Sprach)Kontakt mit dem Tschulymtürkischen standen (und stehen), einer Turksprache (Tuchkova/Kuznetsova 2010). Im Tschulymtürkischen Beispiel (302) ist die finite Verbform kän - 'schicken' mit dem Konverb čättig 'rauchen' verschmolzen und befindet sich somit auf dem Wege zur letzten Stufe des Grammatikalisierungsprozesses 11 - ein aspektuelles Suffix.

(302) Čättig-ip-is-kän.

choke-CVB-send-PST..3SG

'Er rauchte.'

(Lemskaya 2012: 304, Tschulym)

Die Annahme eines turksprachigen Einflusses bietet somit nicht nur eine Erklärung für die generelle Verwendung konverbaler Auxiliarkonstruktionen im Selkupischen, sondern auch für das beobachtete Süd-Nord-Gefälle aufgrund der unterschiedlichen Häufigkeit der Verwendung: Über die Hälfte der Konstruktionen ist in südselkupischen Beispielen belegt (siehe auch Tabelle 30). Mit detaillierten Untersuchungen zu selkupisch-turksprachigen Sprachkontaktphänomenen sollte dieser Fragestellung weiter nachgegangen werden.

Tab. 30: Dialektale Verteilung der Auxiliarverbindungen

\begin{tabular}{cccc}
\hline Nord & Zentral & Zentral/Süd & Süd \\
\hline 7 & 4 & 21 & 41 \\
\hline
\end{tabular}

Endgültige Aussagen lassen sich zum gegenwärtigen Zeitpunkt nicht machen. Eine Vergrößerung der Datenmenge ist ebenso wünschenswert wie eine detailliertere Analyse der konverbalen Konstruktionen (insbesondere der Gruppe D) des Selkup Language Corpus, indem eine Klassifizierung der finiten Verbformen analog zum Tatarischen und Kamassischen vorgenommen wird. Des Weiteren könnte der erweiterte Blick auf ähnliche Konstruktionen in den anderen samojedischen Sprachen (Nenzisch, Enzisch, Nganasanisch, Kamassisch), ebenso wie in Kontaktsprachen wie beispielsweise Tschulymtürkisch neue (Forschungs-) Ergebnisse hervorbringen. Die beschriebenen Aufgaben sprengen jedoch die Möglichkeit der vorliegenden Arbeit und müssen (hoffentlich) zu einem späteren Zeitpunkt realisiert werden.

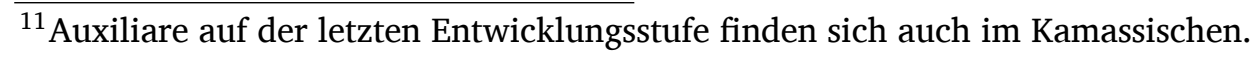





\section{Zusammenfassung und Ausblick}

Das nun folgende letzte Kapitel fasst einerseits die wichtigsten Aspekte der Arbeit noch einmal zusammenfassen und formuliert darüber hinaus Themen(komplexe) bzw. Fragen für eine künftige weitere Erforschung des Selkupischen.

Eingebettet in den von Dixon (1997) entwickelten theoretischen Rahmen der Basic Linguistic Theory (BLT) hatte die Arbeit folgende Ziele: Eine systematische und (vorrangig) synchrone Beschreibung syntaktischer Strukturen des Selkupischen anhand der zentralen und südlichen Dialekte anzufertigen, und den als „Flickenteppich“ bezeichneten Gesamteindruck des strukturellen Systems des Selkupischen zumindest innerhalb der syntaktischen Strukturen zu entwirren und ihm eine erste Form zu geben. Die Entscheidung für die BLT als theoretischen Rahmen beruhte auf dem Wunsch der Verfasserin, den wenig erforschten Bereichen, insbesondere in der selkupischen Syntax, möglichst offen begegnen zu können. Vor dem einschränkenden indoeuropäischen kulturellen und gesellschaftlichen Kontext der Verfasserin sollte soviel theoretischer Rahmen wie nötig und so wenig wie möglich verwendet werden.

Die Beschreibung syntaktischer Strukturen wurde auf der Phrasenebene begonnen und für die strukturell komplexeren Einheiten - wie Teilsatz („clause“) und Satz (,sentence“) - fortgesetzt. Ausgehend von den jeweiligen Phrasenköpfen wurden Nominalphrasen (Seite 19), Adjektivphrasen (Seite 56), Adverbphrasen (Seite 60), Adpositionalphrasen (Seite 62) und Verbphrasen (Seite 76) beschrieben, indem neben den Phrasenköpfen auch sämtliche im Textkorpus belegten Modifikatoren mit Beispielen illustriert wurden. Wie in den meisten uralischen Sprachen geht auch im Selkupischen der Modifikator dem Kopfnomen konsequent voraus. Das gilt nicht nur für Adjektive, Genitiv-NPs und Numerale, sondern auch für Quantifizierer und Partizipien. Zwischen Modifikatoren und ihren Kopfnomen gibt es grundsätzlich keine Kongruenz, eine Ausnahme bildet das emphatische Pronomen on-, für das in den Beispielen (25a) und (25b) auf Seite 34 vollständige Kongruenz belegt werden kann. Nach Numeralen und Quantifizierern erscheint das Kopfnomen generell im Singular (Helimski (1998: 575), Laakso (2011: 192)), Pluralmarkierungen sind nur vereinzelt belegt. Partizipien fungieren im Selkupischen als Modifikator einer NP. Die relativierten Komponenten werden zumeist mit Hilfe einer Relativsatzkonstruktion übersetzt, ohne dass ihnen eine komplexe Satzstruktur zugrundeliegt. Attributive Relativsätze kennt das Selkupische ursprünglich nicht, sie haben sich teilweise unter russischem Einfluß entwickelt und werden als konjunktionale Sätze geformt. Anders als in nordsamojedischen Sprachen gehen selkupische Partizipien keine Kongruenz (hinsichtlich Kasus und Numerus) mit ihrem Bezugsnomen ein. Sie drücken immer aktive Bedeutungen aus. Die von Cheremisina/Martynova (1991) angenommene Möglichkeit, mit Partizipien passivische Bedeutung ausdrücken zu lassen, lässt sich im Textkorpus 


\section{Zusammenfassung und Ausblick}

nicht nachweisen ${ }^{1}$. Wie alle Modifikatoren tritt das Partizip im Zentral- und Südselkupischen stets pränominal auf (siehe A. I. Kuznecova/Chelimskij/Grushkina (1980: 339)), es unterscheidet sich hier vom Nordselkupischen. Eine eindeutige Zuordnung von Partizipien (Präsens und Präteritum) zu den jeweiligen Tempora lässt sich im Textkorpus nicht anhand von Beispielen belegen. Partizip-Präsens-Beispiele können sowohl mit Verben im Präsens als auch im Präteritum stehen und vice versa. Ob an dieser Stelle das ursprüngliche System (PTCP.PRS + Präsens, PTCP.PST + Präteritum) defekt ist, lässt sich aufgrund der zu wenigen Belege nicht eindeutig feststellen.

Innerhalb der Verbphrase wurde gezeigt, dass ein finites Verb Kopf der Phrase ist und in der Regel final erscheint. Die finite Verbform kann eine lexikalische Verbform, ein Modalverb, die Kopula $\bar{e}$ - 'sein' oder ein negatives Existentialverb (čajki - 'NEG.EX') bzw. das negative Hilfsverb (tad'a - 'nicht.können') sein. Die finite Form eines lexikalischen Verbs oder eines Modalverbs kann mit inifiniten Verbformen wie dem Infinitiv oder einem Konverb modifiziert werden. In Beispiel 100a auf Seite 83 konnte eine analytisch gebildete Tempusform gezeigt werden, die es originär im Selkupischen nicht gibt. Das Bildungsmuster ist aus dem Russischen übernommen worden.

Subjekte erscheinen praktisch ausnahmslos unmarkiert, also im Nominativ. GenitivSubjekte in infiniten Konstruktionen (Comrie (1988: 471), Helimski (1998: 576)) lassen sich im Korpus nicht belegen. Etwa zwei Drittel (64\%) aller Subjekte im Textkorpus sind Pronomen, wobei nur 17\% von ihnen overt ausgedrückt werden. Als Pro-Drop-Sprache müssen pronominale Subjekte oder Objekte nicht lexikalisch overt ausgedrückt werden.

Das System der differentiellen Objektmarkierung (DOM) im Selkupischen (Seite 92) ist noch nicht zufriedenstellend erforscht. Die bisher aufgestellten Regeln zur Verwendung markierter und unmarkierter Objekte (Castrén (1854: 140; 309), Prokof'ev (1931: 85-86), Wickman (1955), Helimski (1998: 576) können im Korpus teilweise belegt werden, teilweise finden sich aber auch Gegenbeispiele (wie beispielsweise indirekte Objekte, die mit dem Akkusativ markiert sind wie in Beispiel (126b auf Seite 101 oder Akkusativobjekte mit Imperativen (Abschnitt 4.3) auf Seite 131). Gleiches gilt für die Verwendung der beiden unterschiedlichen Konjugationsarten (in der Arbeit als 'subjektiv' und 'objektiv' bezeichnet) und ihrer Interaktion mit markierten bzw. unmarkierten Objekten. Auch dort entsteht anhand der Beispiele in Kapitel 3.1.2 auf Seite 92 der Einruck, dass sämtliche denkbaren Kombinationen (subjektive Konjugation mit Nominativ- bzw. Akkusativobjekten, objektive Konjugation mit Nominativ- bzw. Akkusativobjekten) möglich sind.

Konstituenten können im Selkupischen trotz einer relativ freien Abfolge zum Zwecke der Topikalisierung, wie in den meisten uralischen Sprachen, satzinitial erscheinen (vgl. hierzu auch Comrie (1988: 469), Helimski (1998: 576), Laakso (2011: 190)). Die Wortfolge von Konstituenten als SOV wird mit den Korpusdaten teilweise bestätigt und ist im Zentralselkupischen auch weitestgehend homogen (abgesehen von anderen Wortfolgen zum Zwecke der Topikalisierung). Die südselkupischen Dialek-

\footnotetext{
${ }^{1}$ Cheremisina/Martynova (1991) postuliert hier Ähnlichkeiten zum Chantischen, wo praktisch alle semantischen Rollen syntaktisch passiviert werden können.
} 
te (und hier insbesondere die Ket'-Subdialekte) bieten dagegen ein uneinheitliches Bild, indem sämtliche denkbaren Wortfolgen auch vorkommen, ohne dass mit ersten Analysen ein konkretes Muster auszumachen ist. Wieder entsteht der Eindruck des Nicht-Intaktseins eines ursprünglichen Systems und dessen Veränderung. Die hier notwendige detailliertere Forschung sprengt den Rahmen der vorliegenden Arbeit bei weitem und muss daher unterbleiben.

Auf der Ebene des einfachen Satzes können die drei Satzmodi Deklarativsatz (Seite 99), Interrogativsatz (Seite 126) und Imperativsatz (Seite 131) unterschieden werden. In allen Satztypen kongruiert die finite Verbform in Numerus und Person mit dem syntaktischen Subjekt. In Deklarativ- und Imperativsätzen werden die Kategorien Person, Numerus, Tempus und/oder Modus morphologisch an der finiten Verbform markiert. In eingeleiteten Interrogativsätzen wurden Kasus und Numerus ursprünglich am Interrogativwort markiert, im Textkorpus sind diese Paradigmen jedoch unvollständig.

In Kapitel 4.1.3 auf Seite 110 wurden eine Vielzahl an Relationen beschrieben, die im Selkupischen mittels Kopulakonstruktionen ausgedrückt werden können. Neben Existenzialsätzen sind das vor allem Lokativ- und Possessivsätze sowie nominale Prädikationen. Die drei Typen weisen die gleiche zugrundeliegende Struktur auf und können lediglich durch die Wortfolge unterschieden werden. Adnominale Possessivität ist dependentmarkiert, der nominale Possessor ist Modifikator einer NP und wird mit dem Genitiv markiert. Vereinzelt können auch Belege für die von Bekker (1995a: 78) beschriebene Lokativmarkierung 2 des Possessors gefunden werden, wie in den Beispielsätzen (35a) - (35c) auf Seite 43. Pronominale Possessoren werden entweder durch ein Pronomonen, ein Possessivsuffix oder eine Kombination von beidem ausgedrückt und ebenfalls mit dem Genitiv oder Adessiv markiert (vgl. hierzu auch Budzisch (2015)). Das Possessum ist zumeist unmarkiert, teilweise erscheint es mit einem Possessivsuffix markiert, das in Person und Numerus mit dem Possessor übereinstimmt (me nenaut - 'unsere Schwester.unsere'). Die Kopula muss in samojedischen Sprachen nicht lexikalisch overt realisiert werden, im Selkupischen findet sich darüber hinaus eine nominale Konjugation, innerhalb derer Personalendungen direkt an Nomina angeschlossen werden können. Hier sei auf die Beispiele $3 a$ und $3 b$ aus der Einleitung auf Seite 16 verwiesen.

Auf der Ebene des komplexen Satzes konnte herausgearbeitet werden, dass es ursprünglich weder Konjunktionen noch Relativpronomen gab und somit weder Subordination noch Koordination konjunktional ausgedrückt werden konnte. Stattdessen wurden komplexe Sachverhalte strukturell einfach umgesetzt, wie Partizipien als Modifikatoren von NPs im Falle von Relativsätzen. Oder es wurden infinite Verbformen wie Konverben, der Infinitiv und Nominalisierungen verwendet, um unterschiedliche ko- und subordinierende Sachverhalte auszudrücken. Die Grundlage eines „clause“ ist im Selkupischen ein Prädikat, das auch infinit sein kann (wie im Falle der Konverben). Es konnte anhand von Beispiel (217) auf Seite 147 gezeigt werden, dass auch

\footnotetext{
${ }^{2}$ In der vorliegenden Arbeit wird der bei Bekker als Lokativ für animate Nomen verwendete Kasus
} -nan als Adessiv bezeichnet. 


\section{Zusammenfassung und Ausblick}

infinite Prädikate ein explizites Subjekt aufweisen können, auch wenn dies bisher nur in wenigen Belegen vorkommt. Nominalisierungen, wie sie noch von Helimski (1998: 576f.) für das Nordselkupische beschrieben sind, lassen sich im zugrundeliegenden Textkorpus weder für zentrale, noch südliche oder nordselkupische Texte belegen. Hier wären weitere Forschungen anhand von zusätzlichem Belegmaterial wünschenswert, um ein besseres Bild von infiniten Satzstrukturen einerseits zu erhalten und von deren Verwendung innerhalb der jeweiligen Dialektgruppen andererseits.

Das System komplexer Sätze wurde und wird unter russischem Einfluss grundlegend umstrukturiert (hierher gehört die Bildung subordinierter und koordinierter Sätze mit Hilfe von entlehnten Konjunktionen, aber auch die Funktionserweiterung von „einheimischen" Wörtern), die Auswirkungen sind insbesondere in den zentralen und südlichen Dialekten sichtbar, wo ein Rahmen in Form einer Schriftsprache nicht vorhanden ist (Helimski (1998: 576), Laakso (2011: 195f.)).

Die Konverben nehmen innerhalb der Arbeit gewissermaßen eine Art Sonderstatus ein. Aus diesem Grund ist ihnen das Exkurskapitel (Seite 185) gewidmet. Der schon verschiedentlich genannte „Flickenteppich“ lässt sich hier besonders eindrucksvoll beobachten. Weiter oben wurden Konverben bereits als Mittel zur Satzkoordination bzw. -subordination genannt. Zusätzlich wurde in Kapitel 6 auf Seite 185 gezeigt, dass Konverben im Selkupischen nicht nur komplexe Prädikate bilden, mit den komplexe Bewegungen ausgedrückt werden können, sondern dass sie überdies auch in Auxiliarverbindungen verwendet werden. In letzteren ist das Konverb Träger der lexikalischen Bedeutung, während die finite Verbform unterschiedlich weit „desemantisiert“ ist und wie ein Hilfsverb als Träger der grammatischen Informationen dient. In den südselkupischen Dialekten (insbesondere in den Ket'-Subdialekten) sind Konstruktionen dieser Art vielfach vorhanden, in den zentralen und nördlichen Dialekten gibt es sie nur selten bis vereinzelt. Da das syntaktische Verhalten von Konverben im Selkupischen bisher nicht adäquat beschrieben worden ist, wäre auch hier weitere Forschung überaus sinnvoll. Hier sind insbesondere auch Einflüsse durch Sprachkontakt interessant (beispielsweise turksprachige Einflüsse auf die südselkupischen Dialekte).

Die Betrachtung von negierten Phrasen und Sätzen hat unter anderem gezeigt, dass Belege mit negierten infiniten Konstruktionen (wie Konverben) fast völlig fehlen. Auch hier ist weitere Forschung nötig, um Antworten zu finden. Weiterhin hat das Selkupische die ursprüngliche samojedische Negation in Form eines Negationsverb in der Standardnegation eingebüßt. Lediglich das Matorische verfügte ebenfalls über eine Standardnegation mittels einer Negationspartikel (Mikola (2004: 128)). Stattdessen erfolgt die Standardnegation mit Hilfe einer entlehnten Negationspartikel (vgl. auch Wagner-Nagy (2011: 65)). Im Unterschied zu zentralen und südlichen Dialekten (hier wird in allen Tempora durchgängig die Negationspartikel verwendet) wird im Nordselkupischen im Präteritum Negation auch mit Hilfe einer nominalisierten Verbform und dem negativen Existenzialverb ausgedrückt. Im Imperativ verfügt das Selkupische über eine spezielle negative Imperativpartikel, Existenzial-, Lokativ- und Possessivsätze werden mit Hilfe eines negativen Existenzialverbs negiert. Innerhalb der komplexen Sätze ist auffällig, dass nur finite Konstituentensätze negiert werden. Infinite Konstitutentensätze, die eine konverbale Form oder einen Infinitiv enthalten, 
erscheinen stets in affirmativer Form.

Die Schwierigkeiten bei der Beschreibung der grundlegenden syntaktischen Strukten im Selkupischen sind vielfältig. Das begrenzte Sprachmaterial mit Einschränkungen hinsichtlich Sprecheranzahl und -geschlecht, einer ungleichen Genreverteilung, das zudem nur in Form von Texten vorliegt, stellt einen wichtigen Punkt dar. Das Korpus gibt darüber hinaus die gesprochene selkupische Sprache wieder (auch wenn sie in Texten manifestiert ist). Als Folge findet sich eine enorme dialektale Vielfalt, die es erlaubt, die Sprache quasi bei einzelnen Sprechern beobachten zu können. Allerdings verhindert genau diese Differenziertheit einen weiter gefassten Blick auf vorhandene Strukturen. Allgemeingültige Aussagen lassen sich nur schwer treffen, da viele Phänomene lediglich bei einem Sprecher oder in einzelnen Texten belegt sind. Das macht sie im Grunde nicht weniger wahrscheinlich, doch die Verifizierung kann (auch in Ermangelung von Sprechern) nicht erfolgen.

Dennoch ist es mit der vorliegenden Arbeit gelungen, syntaktische Strukturen gerade für die wenig dokumentierten zentralen und südlichen Dialekte des Selkupischen grundlegend darzustellen. Es bleibt zu wünschen, dass von dieser Arbeit ausgehend die verschiedenen Bereiche (nicht nur der Syntax) weiter erforscht werden, um die vielen noch existierenden Lücken im Wissen um das Selkupische zu schließen. Bis es soweit ist, bleibt bei der Auseinandersetzung mit der Vielfältigkeit der Strukturen oft nur das Hinnehmen und Akzeptieren:

„M’i čumilqummut nid’ik mēkkaut“ (Wir Selkupen machen es so.) 



\section{A Graphemübersicht}

\begin{tabular}{|c|c|c|c|c|c|}
\hline DissAH & Unicode & IPA & Bykonja 2005 & Dul'zon 1966a & Kuz'mina 1974 \\
\hline $\mathrm{a}$ & 0061 & $\mathrm{a}$ & $\mathrm{a}$ & a & a \\
\hline$\overline{\mathrm{a}}$ & $0061+$ & a: & $\bar{a}$ & a: & a: \\
\hline e & 0065 & $\mathrm{e}$ & $\mathrm{e}$ & $\mathrm{e}$ & e, $\mathrm{e}^{1}, \partial$, \\
\hline$\overline{\mathrm{e}}$ & $0065+0304$ & e: & $\overline{\mathrm{e}}$ & e: & e: \\
\hline$\varepsilon$ & 025B & $\varepsilon$ & э & & $\varepsilon \sqrt[3]{3}$ \\
\hline $\bar{\varepsilon}$ & $025 B+0304$ & $\varepsilon:$ & $\bar{\jmath}$ & & $\varepsilon: 4$ \\
\hline$\ddot{a}$ & $00 \mathrm{E} 6$ & $æ$ & $\ddot{a}$ & $\ddot{a}$ & $æ$ \\
\hline$\overline{\ddot{a}}$ & $00 \mathrm{E} 6+0304$ & æ: & $\overline{\ddot{a}}$ & ä: & æ: \\
\hline $\mathrm{i}$ & 0069 & $\mathrm{i}$ & и, $\dot{\mathrm{u}}$ & и; і & $\mathrm{i}$ \\
\hline$\overline{1}$ & $0069+0304$ & i: & $\overline{\mathbf{U}}$ & и:; i: & $\mathrm{i}:, \mathrm{I}^{5}$ \\
\hline I & 026A & I & и & & I \\
\hline I: & $026 \mathrm{~A}+0304$ & I: & $\overline{\mathbf{n}}$ & & \\
\hline$\dot{\mathbf{q}}$ & 0268 & $\dot{\mathbf{i}}$ & ы & Ы & $\dot{\mathbf{i}}$ \\
\hline$\overline{\mathbf{f}}$ & $0268+0304$ & i: & $\overline{\mathbf{b}}$ & ы: & i: \\
\hline ə & 0259 & $\partial$ & $\ddot{3} ;$ ъ & $\mathbf{b}$ & $\partial$ \\
\hline $\bar{\partial}$ & $0259+0304$ & ว: & $\ddot{3}$ & & ว:, æ: \\
\hline o & 006F & o & o & o & \\
\hline$\overline{0}$ & $006 F+0304$ & o: & $\overline{0}$ & o: & $0: ; \Lambda: 0^{6}$ \\
\hline כ & $006 \mathrm{~F}$ & כ & 0 & & כ \\
\hline כ: & $006 F+0304$ & ว: & $\overline{0}$ & & ว:; о: \\
\hline$\ddot{0}$ & 00F6 & $\varnothing$ & $\ddot{0}$ & $\ddot{0}$ & $\emptyset ; œ$ \\
\hline ö: & $00 \mathrm{~F} 6+0304$ & $\emptyset:$ & $\overline{\ddot{O}}$ & ö: & $\emptyset:$ \\
\hline $\mathrm{u}$ & 0075 & $\mathrm{u}$ & $\mathrm{y}$ & $\mathrm{y}$ & $\mathrm{u}$ \\
\hline $\mathrm{u}:$ & $0075+0304$ & $\mathrm{u}$ & $\overline{\mathrm{y}}$ & $y:$ & $\mathrm{u}:$ \\
\hline$\ddot{\mathrm{u}}$ & OOFC & $\mathrm{y}$ & $\ddot{\mathrm{y}}$ & $\ddot{\mathrm{y}}$ & $\mathrm{y}$ \\
\hline ü: & $00 F C+0304$ & $y:$ & $\overline{\overline{\mathrm{y}}}(\mathrm{C}) ; \overline{\overline{\mathrm{o}}}\left(\mathrm{C}^{\prime}\right)$ & $\ddot{y}:$ & $y:$ \\
\hline $\mathrm{d}$ & 0064 & $\mathrm{~d}$ & д & д & \\
\hline
\end{tabular}

\footnotetext{
${ }^{1}$ Früher waren [e] und [ĕ] verschiedene Phoneme, heutzutage (also 1970er Jahre) nicht mehr (Kuzmina 1974: 144).

${ }^{2}$ Erscheint als Bindevokal, unbetont.

${ }^{3}$ Etwas offener als das lange $\varepsilon$ :.

${ }^{4}$ Kommt selten vor.

${ }^{5}$ Früher waren [i:] und [r:] verschiedene Phoneme, heutzutage (also 1970er Jahre) nicht mehr (Kuzmina 1974: 144).

${ }^{6}{ }_{\Lambda: ;}$ o: waren früher ebenfalls Phoneme (ebd.: 148 ).
} 


\begin{tabular}{|c|c|c|c|c|c|}
\hline DissAH & Unicode & IPA & Bykonja 2005 & Dul'zon 1966a & Kuz'mina 1974 \\
\hline$d^{\prime}$ & $0064+02 B C$ & $\mathfrak{f}$ & дь & д’ & 司 \\
\hline $\mathrm{t}$ & 0074 & $\mathrm{t}$ & $\mathrm{T}$ & $\mathrm{T}$ & $\mathrm{t} ; \mathrm{d}$ \\
\hline $\mathrm{t}^{\prime}$ & $0074+02 B C$ & $\mathrm{t}^{\mathrm{j}}$ & Tb & $\mathrm{T}^{\prime}$ & \\
\hline $\mathrm{m}$ & 006D & $\mathrm{m}$ & M & M & $\mathrm{m}$ \\
\hline $\mathrm{m}^{\prime}$ & $006 \mathrm{D}+02 \mathrm{BC}$ & $\mathrm{m}^{\mathrm{j}}$ & Mь & M’ & \\
\hline $\mathrm{n}$ & 0144 & $\mathrm{n}$ & $\mathbf{H}$ & $\mathbf{H}$ & \\
\hline $\mathrm{n}^{\prime}$ & $0144+02 B C$ & $\mathrm{n}$ & Нь & $\mathrm{H}^{\prime}$ & $\mathrm{n}$ \\
\hline $\mathrm{n}$ & $006 \mathrm{E}$ & $\mathrm{n}$ & $\mathbf{H}$ & & $\mathrm{n}$ \\
\hline$\eta$ & 014B & $\eta$ & $\mathrm{H}$ & $\mathrm{H \Gamma}$ & $\eta$ \\
\hline W & 0077 & $\mathrm{~V}$ & B & B & $\mathrm{V}$ \\
\hline W & 0077 & $\beta$ & & & $\beta$ \\
\hline $\mathrm{p}$ & 0070 & $\mathrm{p}$ & $\Pi$ & $\Pi$ & $\mathrm{p} ; \mathrm{b}$ \\
\hline $\mathrm{z}$ & 007A & $\mathrm{z}$ & 3 & 3 & \\
\hline s & 0073 & $s$ & $\mathrm{c}$ & $c$ & $\mathrm{~s} ; \mathrm{z}$ \\
\hline$\check{S}$ & 0161 & $\int^{j}$ & ш & ш & $\int^{\prime} ; 3^{\prime}$ \\
\hline$\check{c}$ & 010D & $\widetilde{\mathrm{t} J}$ & ч & ч & $\mathrm{t} \int^{\prime} ; \mathrm{d} 3$; $\overline{\mathrm{ts}}$ \\
\hline$\check{c}^{\prime}$ & $010 \mathrm{D}+02 \mathrm{BC}$ & $\widetilde{\mathrm{t} \int^{\mathrm{j}}}$ & чь & ч' & \\
\hline 3 & 0292 & 3 & ж & ж & \\
\hline$\check{3}$ & $01 \mathrm{EF}$ & $\widehat{\mathrm{d} 3}$ & ж & дж & \\
\hline 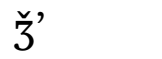 & $01 \mathrm{EF}+02 \mathrm{BC}$ & $\mathrm{d}^{j}$ & жь & & \\
\hline 1 & 006C & 1 & л & л; 1 & $1 ; 1 ; 1$ \\
\hline ' & $006 \mathrm{C}+02 \mathrm{BC}$ & $\Lambda$ & ль & л'; l' & $\Lambda$ \\
\hline $\mathrm{r}$ & 0072 & $\mathrm{r}$ & $\mathrm{p}$ & $\mathrm{p}$ & $\mathrm{r} ; \mathrm{R}$ \\
\hline tw & $0074+0077$ & $\mathrm{t}^{\mathrm{w}}$ & & & $\mathrm{t} \beta$ \\
\hline sw & $0073+0077$ & $\mathrm{~s}^{\mathrm{w}}$ & & & $s \beta$ \\
\hline $\mathrm{j}$ & 006A & $\mathrm{j}$ & Й & $\ddot{\text { Й}}$ & $\mathrm{j}$ \\
\hline$g$ & 0067 & $g$ & $\Gamma$ & $\Gamma$ & \\
\hline $\mathrm{k}$ & 006B & $\mathrm{k}$ & $\kappa$ & К & $\mathrm{k} ; \mathrm{g}$ \\
\hline kw & $006 \mathrm{~B}+0077$ & $\mathrm{k}^{\mathrm{w}}$ & $\mathrm{s}$ & $\mathrm{k} \beta ; \mathrm{kv}$ & \\
\hline $\mathrm{q}$ & 0071 & $\mathrm{q}$ & $\kappa$ & $\mathrm{k}_{\mathrm{g}}$ & $\mathrm{q}$ \\
\hline $\mathrm{q}$ & 0071 & $\mathrm{G}$ & $\pi^{8}$ & $\Gamma 9^{9}$ & \\
\hline qw & $0071+0077$ & $\mathrm{q}^{\mathrm{w}}$ & & $\mathrm{q} \beta$ & \\
\hline $\mathrm{y}$ & 006B & $\mathrm{k}^{\mathrm{w}}$ & $\mathrm{Y}^{10}$ & & \\
\hline $\begin{array}{l}\mathrm{h} \\
?\end{array}$ & 0068 & $\mathrm{x}$ & $\begin{array}{l}\gamma, x^{11} \\
\hbar s ́\end{array}$ & & \\
\hline
\end{tabular}

${ }^{7}$ Wird bei Kuzmina als zentraler, palataler Plosiv beschrieben. ${ }^{8}$ Sth. uvularer Plosiv.

${ }^{9}$ Sth. uvularer Plosiv ( = sth. q).

${ }^{10}$ Stl. uvularer Plosiv (qw).

${ }^{11}$ Uvular, nicht velar. 


\section{B Textsammlungen}

\begin{tabular}{|c|c|c|c|c|c|}
\hline Textname & Genre & Publikation & Dialekt & Subdialekt & Tokens \\
\hline AAI_1973_Okyle_flk & flk & Kuznecova et al. 1993: 9-11 & $\mathrm{N}$ & Middle Taz & 827 \\
\hline APA_1977_Palna_flk & flk & Kuznecova et al. 1993: 32-33 & $\mathrm{N}$ & Taz & 77 \\
\hline AVA_1973_Ichakicha_flk & flk & Kuznecova et al. 1993: 13-15 & $\mathrm{N}$ & Middle Taz & 484 \\
\hline AVA_1973_Ichakicha2_flk & flk & Kuznecova et al. 1993: $17-20$ & $\mathrm{~N}$ & Middle Taz & 1089 \\
\hline BEP_1973_Fat1_flk & flk & Kuznecova et al. 1993: $26-27$ & $\mathrm{~N}$ & Taz & 159 \\
\hline BEP_1973_IchaAndNenets_flk & flk & Kuznecova et al. 1993: $25-26$ & $\mathrm{~N}$ & Taz & 181 \\
\hline BEP_1973_Itja1_flk & flk & Kuznecova et al. 1993: 24 & $\mathrm{~N}$ & Taz & 67 \\
\hline BEP_1973_Itja2_flk & flk & Kuznecova et al 1993: 22-23 & $\mathrm{N}$ & Taz & 97 \\
\hline BEP_1977_Itja3_flk & flk & Kuznecova et al. 1993: 63 & $\mathrm{~N}$ & Taz & 212 \\
\hline BEP_1977_Itja4_flk & flk & Kuznecova et al. 1993: $24-25$ & $\mathrm{~N}$ & Taz & 213 \\
\hline BEP_1977_Itja5_flk & flk & Kuznecova et al. 1993: 24 & $\mathrm{~N}$ & Taz & 141 \\
\hline BEP_1977_OldWoman_flk & flk & Kuznecova et al. 1993: 68-69 & $\mathrm{N}$ & Taz & 150 \\
\hline BIV_1941_KonMytyke_flk & flk & Tučkova/Wagner-Nagy 2015: 198-201 & $\mathrm{N}$ & Taz & 222 \\
\hline BVP_1973_East_flk & flk & Kuznecova et al. 1993: 8-9 & $\mathrm{N}$ & Taz & 351 \\
\hline KaIA_1973_Natenka_flk & flk & Kuznecova et al. 1993: 12-13 & $\mathrm{N}$ & Taz & 531 \\
\hline KMP_1971_Ichkyta_flk & flk & $\begin{array}{l}\text { Kuznecova et al. 1993: 15-17 } \\
\text { Tučkova/Wagner-Nagy 2015: 30-39 }\end{array}$ & $\mathrm{N}$ & Taz & 926 \\
\hline KMP_1971_NyomalPorky & flk & Kuznecova et al. 1993: 30-31 & $\mathrm{N}$ & Taz & 514 \\
\hline KVM_1977_IchaAndNenets_flk & flk & Kuznecova et al. 1993: 26 & $\mathrm{~N}$ & Taz & 143 \\
\hline MA_1957_BearAndHare_flk & flk & Lehtisalo 1960: 337 & $\mathrm{~N}$ & Taz & 100 \\
\hline MA_1957_Fox_flk & flk & Lehtisalo 1960: 337 & $\mathrm{~N}$ & Taz & 55 \\
\hline MIV_1977_Icha_flk & flk & Kuznecova et al. 1993: 20-22 & $\mathrm{N}$ & Upper Taz & 433 \\
\hline MIV_1977_Nyomal Porky_flk & flk & Kuznecova et al. 1993: 31-32; 74-75 & $\mathrm{N}$ & Upper Taz & 256 \\
\hline MIV_1977_TwoBrothers_flk & flk & Kuznecova et al. 1993: 33-34 & $\mathrm{N}$ & Upper Taz & 253 \\
\hline NN_1973_Shaman_flk & flk & Kuznecova et al. 1993: 34 & $\mathrm{~N}$ & Taz & 66 \\
\hline NN_1977_OldMan_flk & flk & Kuznecova et al. 1993: 28 & $\mathrm{~N}$ & Taz & 147 \\
\hline NN_1977_OldManAndOldWoman_flk & flk & Kuznecova et al. 1993: 28 & $\mathrm{~N}$ & Taz & 123 \\
\hline AGS_1967_FairytaleSnake_flk & flk & Kuz'mina1967: 294-296|317-318 & $\mathrm{S}$ & Middle Ket' & 746 \\
\hline BAG_1964_ItjaMousetrapped_flk & flk & Tučkova/Wagner-Nagy 2015: 76 & $\mathrm{~S}$ & Upper Ket' & 30 \\
\hline BNN_1971_DogSearchedMistress_flk & flk & Becker 1980: 57-59 & $\mathrm{S}$ & Middle Ket' & 263 \\
\hline BNN_1971_EyesEars_flk & flk & Becker 1980: 55-57 & $\mathrm{S}$ & Middle Ket' & 212 \\
\hline ILP_1981_HowSelkupLivedBefore_nar & nar & Bykonia et al 1996: 83-85, 163-165 & $\mathrm{S}$ & Middle Ob & 94 \\
\hline ILP_1981_ItjaGrandmother_flk & flk & Bykonia et al 1996: 32, 135 & $S$ & Middle Ob & 58 \\
\hline ILP_1981_ItjaGrandmother_flk & & Tučkova/Wagner-Nagy 2015: 140 & & & \\
\hline ILP_1981_ItjaOneEyedDevil_flk & flk & $\begin{array}{l}\text { Bykonia et al 1996: 35-37, } 137 \\
\text { Tučkova/Wagner-Nagy 2015: } 112-113\end{array}$ & $S$ & Middle Ob & 117 \\
\hline ILP_1981_ItjaPoenegesse_flk & flk & $\begin{array}{l}\text { Bykonia et al 1996: 29, } 134 \\
\text { Tučkova/Wagner-Nagy 2015: } 106\end{array}$ & $S$ & Middle Ob & 60 \\
\hline ILP_1981_Poenegesse_flk & flk & $\begin{array}{l}\text { Bykonia et al 1996: 25-26, 131-132 } \\
\text { Tučkova/Wagner-Nagy 2015: 114-116 }\end{array}$ & $S$ & Middle Ob & 190 \\
\hline ILP_1981_StonyOldWoman_flk & flk & Bykonia et al 1996: 39, 138 & $S$ & Middle Ob & 60 \\
\hline KKA_NN_HazelgrouseMouse_flk & flk & Morev et al 1981: 140 & $\mathrm{~S}$ & Upper Ket' & 106 \\
\hline KKN_1971_FiveCarpBrothers_flk & flk & $\begin{array}{l}\text { Becker 1980:59-64 } \\
\text { Tučkova/Wagner-Nagy 2015: 265-270 }\end{array}$ & $S$ & Upper Ket' & 502 \\
\hline KKN_1971_Itja_flk & flk & $\begin{array}{l}\text { Becker 1980:68-71 } \\
\text { Tučkova/Wagner-Nagy 2015: 107-111 }\end{array}$ & $S$ & Upper Ket' & 427 \\
\hline KKN_1971_IvanAndGod_flk & flk & Becker 1980: 64-66 & $\mathrm{S}$ & Upper Ket' & 325 \\
\hline KKN_1971_WhiteHeadedWoman_flk & flk & Becker 1980: 66-68 & $\mathrm{S}$ & Upper Ket' & 140 \\
\hline KMS_1963_Bear_flk & flk & Kuz'mina1967: 292|316 & $\mathrm{S}$ & Middle Ket' & 71 \\
\hline KMS_1963_HazelgrouseMouse_flk & flk & Kuz'mina1967: 291-292|315-316 & $S$ & Middle Ket' & 239 \\
\hline KMS_1963_Sluggard_flk & flk & Dul'zon 1966a: 122|131 & $\mathrm{S}$ & Middle Ket' & 92 \\
\hline KMS_1963_UnknownMan_flk & flk & Kuz'mina1967: 290-291|314 & $\mathrm{S}$ & Middle Ket' & 153 \\
\hline
\end{tabular}




\section{B Textsammlungen}

\begin{tabular}{|c|c|c|c|c|c|}
\hline Textname & Genre & Publikation & Dialekt & Subdialekt & Token \\
\hline KMS_1966_MouseGray_flk & flk & Dul'zon 1966a: 116,118|125,127 & $S$ & Middle Ket' & 142 \\
\hline KMS_1966_TwoSisters_flk & flk & $\begin{array}{l}\text { Dul'zon 1966a: } \\
118,120,122 \mid 127,129,131\end{array}$ & $S$ & Middle Ket' & 573 \\
\hline KMS_1967_Antosja_trans & trans & Kuz'mina1967: 285-286|310-311 & $S$ & Middle Ket' & 339 \\
\hline KMS_1967_EagleOwl_nar & nar & Kuz'mina1967: 292|316 & $\mathrm{S}$ & Middle Ket' & 64 \\
\hline KMS_1967_Elk_nar & nar & Kuz'mina1967: 293|316 & $\mathrm{S}$ & Middle Ket' & 46 \\
\hline KMS_1967_Fire_nar & nar & Kuz'mina1967: 293|316 & $\mathrm{S}$ & Middle Ket' & 116 \\
\hline KMS_1967_Hunt_trans & trans & Kuz'mina1967: 287-290|311-314 & $\mathrm{S}$ & Middle Ket' & 1000 \\
\hline KMS_1968_Burbot_trans & trans & Kuz'mina1967: 278-281|303-305 & $\mathrm{S}$ & Middle Ket' & 922 \\
\hline KNI_1964_ItjaCapeOfWorms_flk & flk & $\begin{array}{l}\text { Tučkova/Helimski 2010: 99-104 } \\
\text { Tučkova/Wagner-Nagy 2015: 206-210 }\end{array}$ & $\mathrm{S}$ & Upper Ket' & 352 \\
\hline MMP_1964_Lgov_trans & trans & Kuz'mina1967: 268-273|297-300 & $\mathrm{S}$ & Middle Ket' & 804 \\
\hline MPF_1964_Birjuk_trans & trans & Kuz'mina1967: 274-276|301-303 & $\mathrm{S}$ & Middle Ket' & 333 \\
\hline NN_1879_Itja_flk & flk & Künnap1992: 141-147 & $\mathrm{S}$ & $\begin{array}{l}\text { Lower Cha- } \\
\text { ja }\end{array}$ & 742 \\
\hline NN_1913_Itja_flk & flk & $\begin{array}{l}\text { Tučkova/Wagner-Nagy 2015: 82-88 } \\
\text { Grigorovskij 1879:30-33 } \\
\text { Katz 1988:24-33 }\end{array}$ & S & $\begin{array}{l}\text { Lower Cha- } \\
\text { ja }\end{array}$ & 535 \\
\hline NN_NN_IWentFishing_nar & nar & Bykonia et al 1996: $65 \mid 155$ & $S$ & Middle Ob & 65 \\
\hline PES_1964_Lgov_trans & trans & Kuz'mina1967: 268-273|297-300 & $S$ & Lower Ket' & 158 \\
\hline PFN_1964_Birjuk_trans & trans & Kuz'mina1967: 276-277|301-303 & $\mathrm{S}$ & Upper Ket' & 280 \\
\hline PMP_1961_Fairytale_flk & flk & Kuz'mina1967: 321-326|328-329 & $\mathrm{S}$ & Middle Ob & 1584 \\
\hline PMP_1961_ForestWoman_flk & flk & $\begin{array}{l}\text { Dul'zon 1996a: } \\
136,38,40,42,44,46,48,50 \mid 45,47,49,51- \\
55\end{array}$ & $\mathrm{~S}$ & Middle Ob & 2103 \\
\hline PMP_1966_BodylessHead_flk & flk & $\begin{array}{l}\text { Dul'zon 1996a: } \\
\text { 128,130,132,134|137,139,141 }\end{array}$ & S & Middle Ob & 560 \\
\hline PMP_1966_BoyDevil_flk & flk & Dul'zon 1996a: $134,136 \mid 141,143$ & $\mathrm{~S}$ & Middle Ob & 393 \\
\hline PMP_1967_AboutItja_flk & flk & Tučkova/Wagner-Nagy 2015: 64-69 & $\mathrm{S}$ & Middle Ob & 570 \\
\hline PVD_1961_FarmAssault_flk & flk & $\begin{array}{l}\text { Dul'zon 1996a: } \\
122,124,126,128 \mid 131,133,135\end{array}$ & $\mathrm{~S}$ & Upper Ob & 705 \\
\hline PVD_1966_BoyDevil_flk & flk & Dul'zon 1996a: $128 \mid 137$ & $\mathrm{~S}$ & Upper Ob & 202 \\
\hline SEV_1980_HazelGrouse_flk & flk & $\begin{array}{l}\text { Bykonia et al 1996: 41-59 } \\
\text { Bajdak et al 2010: 133-162 } \\
\text { Bajdak/Maksimova 2009: } 45-89\end{array}$ & $\mathrm{~S}$ & Middle Ob & 416 \\
\hline SEV_1981_SisterBrother_flk & flk & $\begin{array}{l}\text { Bykonia et al 1996: 9-23 } \\
\text { Bajdak et al 2010: } 163-183\end{array}$ & $\mathrm{~S}$ & Middle $\mathrm{Ob}$ & 631 \\
\hline SMS_1980_ItjaForest_flk & flk & Tučkova/Wagner-Nagy 2015: 120-121 & $\mathrm{S}$ & Middle Ob & 164 \\
\hline SVG_1964_IitekaPinaeweldju_flk & flk & $\begin{array}{l}\text { Tučkova/Helimski 2010: 88-92 } \\
\text { Tučkova/Wagner-Nagy 2015: 89-92 }\end{array}$ & $\mathrm{S}$ & Upper Ket' & 304 \\
\hline SVG_1964_ItjeFatherFishMotherFish_flk & flk & $\begin{array}{l}\text { Tučkova/Helimski 2010: 92-98 } \\
\text { Tučkova/Wagner-Nagy 2015: 215-220 }\end{array}$ & $\mathrm{S}$ & Upper Ket' & 498 \\
\hline SVG_1964_Stars_flk & flk & $\begin{array}{l}\text { Tučkova/Wagner-Nagy 2015: 195-196 } \\
\text { Kuzmina 1977:75 }\end{array}$ & S & Upper Ket' & 63 \\
\hline SVG_1967_Spring_trans & trans & Kuz'mina1967: 277|303 & $\mathrm{S}$ & Upper Ket' & 50 \\
\hline TET_1979_HowBeastsSearchedSun_flk & flk & Morev et al 1981: 143 & $\mathrm{~S}$ & Middle Ket' & 49 \\
\hline TET_1979_SnowGirl_flk & flk & Morev et al 1981: 142-143 & $\mathrm{S}$ & Middle Ket' & 90 \\
\hline TET_1979_TaleFoxDōofflk & flk & Morev et al 1981: 141 & $\mathrm{~S}$ & Middle Ket' & 64 \\
\hline TET_1979_TheHaresHouse_flk & flk & Morev et al 1981: 141-142 & $\mathrm{S}$ & Middle Ket' & 124 \\
\hline TFF_1967_ItjaAldigaNet_flk & flk & Tučkova/Wagner-Nagy 2015: 74-75 & $\mathrm{S}$ & Middle Ob & 186 \\
\hline TFF_1967_ItjaAldigaScale_flk & flk & Tučkova/Wagner-Nagy 2015: 70-73 & $\mathrm{S}$ & Middle Ob & 349 \\
\hline TFF_1967_ItjaHuntsElk_flk̄ & flk & Tučkova/Wagner-Nagy 2015: 194 & $\mathrm{~S}$ & Middle $\mathrm{Ob}$ & 62 \\
\hline TFF_1967_ItjeAldigaPoenegesse_flk & flk & Tučkova/Wagner-Nagy 2015: 141-46 & $\mathrm{S}$ & Middle Ob & 590 \\
\hline TFF_1967_KolobokkaPoenegessa_flk & flk & Tučkova/Wagner-Nagy 2015: 100-103 & $\mathrm{S}$ & Middle Ob & 313 \\
\hline TMR_1967_Poenege_flk & flk & Tučkova/Wagner-Nagy 2015: 104-105 & $\mathrm{S}$ & Middle Ob & 111 \\
\hline TMR_1981_AboutItja_flk & flk & $\begin{array}{l}\text { Bykonia et al 1996: 61, } 154 \\
\text { Tučkova/Wagner-Nagy 2015: 192-193 }\end{array}$ & $\mathrm{S}$ & Middle Ob & 150 \\
\hline TMR_1981_Devil_flk & flk & Bykonia et al 1996: 69-70, 157-158 & $\mathrm{S}$ & Middle Ob & 192 \\
\hline TMR_1981_Robbers_flk & flk & Bykonia et al 1996: 77-80, 160-162 & $\mathrm{S}$ & Middle Ob & 263 \\
\hline TMR_1981_SapSapiha_flk & flk & Bykonia et al 1996: 73, 159 & $\mathrm{~S}$ & Middle Ob & 66 \\
\hline ZIP_1964_Clock1_trans & trans & Kuz'mina1967: 282-285|306-309 & $\mathrm{S}$ & Middle Ket' & 205 \\
\hline ZIP_1964_Clock2_trans & trans & Kuz'mina1967: 282-285|306-309 & $S$ & Middle Ket' & 158 \\
\hline ZNP_1964_Clock3_trans & trans & Kuz'mina1967: 282-285|306-309 & $S$ & Middle Ket' & 605 \\
\hline
\end{tabular}




\begin{tabular}{|c|c|c|c|c|c|}
\hline Textname & Genre & Publikation & Dialekt & Subdialekt & Tokens \\
\hline ChDN_1983_BearCameIntoVillage_nar & nar & Bajdak/Maksimova 2002: 23 & $\mathrm{Z}$ & Vasjugan & 151 \\
\hline ChDN_1983_GirlAndIce_flk & flk & $\begin{array}{l}\text { Bajdak/Maksimova 2002: 7-8 } \\
\text { Bajdak/Maksimova 2013: 153-169 }\end{array}$ & $\mathrm{Z}$ & Vasjugan & 232 \\
\hline ChDN_1983_HerosDaughter_flk & flk & Bajdak/Maksimova 2002: 17-19 & $\mathrm{Z}$ & Vasjugan & 377 \\
\hline ChDN_1983_ItjaStayedAlone_flk & flk & $\begin{array}{l}\text { Bajdak/Tučkova 2004:61-62 } \\
\text { Tučkova/Wagner-Nagy 2015: 117-119 }\end{array}$ & $\mathrm{Z}$ & Vasjugan & 250 \\
\hline ChDN_1983_ItjasTown_flk & flk & $\begin{array}{l}\text { Bajdak/Tučkova 2004:64 } \\
\text { Tučkova/Wagner-Nagy 2015: 190-191 }\end{array}$ & $\mathrm{Z}$ & Vasjugan & 143 \\
\hline ChDN_1983_MistressOfFire_flk & flk & $\begin{array}{l}\text { Bajdak/Maksimova 2002: 27-37 } \\
\text { Bajdak/Maksimova 2012: 72-99 }\end{array}$ & $\mathrm{Z}$ & Vasjugan & 416 \\
\hline ChDN_1983_MotherInLaw_nar & nar & Bajdak/Maksimova 2015: 108-113 & $\mathrm{Z}$ & Vasjugan & 95 \\
\hline ChDN_1983_Nikita_flk & flk & $\begin{array}{l}\text { Bajdak/Tučkova 2004:62-64 } \\
\text { Tučkova/Wagner-Nagy 2015: 93-96 }\end{array}$ & $\mathrm{Z}$ & Vasjugan & 434 \\
\hline ChDN_1983_Pora_flk & flk & Bajdak/Maksimova 2002: 12-13 & $\mathrm{Z}$ & Vasjugan & 278 \\
\hline ChDN_1983_TaleB̈rownies_flk & flk & Bajdak/Maksimova 2013: 185-200 & $\mathrm{Z}$ & Vasjugan & 218 \\
\hline JIF_1968_Kamacha_flk & flk & Kuz'mina1967: 319|327 & $\mathrm{Z}$ & Tym & 246 \\
\hline KAO_1912_SmartPeople_trans & trans & Katz 1975: 96-108 & $\mathrm{Z}$ & Tym & 167 \\
\hline KFN_1967_BigBear_flk & flk & $\begin{array}{l}\text { Kuz'mina1967: 320|328 } \\
\text { Bajdak/Tučkova 2004:59 } \\
\text { Tučkova/Wagner-Nagy 2015: } 197 \\
\text { Kuzmina1977:76 }\end{array}$ & $\mathrm{Z}$ & Tym & 57 \\
\hline KFN_1967_HumanSizedPike_flk & flk & Kuz'mina1967: 320|328 & $\mathrm{Z}$ & Tym & 82 \\
\hline KFN_1967_Mammoth_flk & flk & Kuz'mina1967: 320|328 & $\mathrm{Z}$ & Tym & 51 \\
\hline KIA_2014_BraveSamoka_trans & trans & Korobejnikova 2014: 123-125 & $\mathrm{Z}$ & Narym & 277 \\
\hline KIA_2014_GiantZobel_trans & trans & Korobejnikova 2014: 55-61 & $\mathrm{Z}$ & Narym & 816 \\
\hline KIA_2014_GrandmotherSolomanida_trans & trans & Korobejnikova 2014: 11-12 & $\mathrm{Z}$ & Narym & 158 \\
\hline KIA_2014_HeroKenga_trans & trans & Korobejnikova 2014: 14-17 & $\mathrm{Z}$ & Narym & 639 \\
\hline KIA_2014_ItjaKet_trans & trans & Korobejnikova 2014: 36-45 & $\mathrm{Z}$ & Narym & 1217 \\
\hline KIA_2014_KatManPuch_trans & trans & Korobejnikova 2014: 61-65 & $\mathrm{Z}$ & Narym & 617 \\
\hline KIA_2014_SelkupBear_trans & trans & Korobejnikova 2014: 128-129 & $\mathrm{Z}$ & Narym & 180 \\
\hline MNN_1977_Hunt_nar & nar & Morev et al 1981: 139 & $\mathrm{Z}$ & Tym & 120 \\
\hline MNN_1977_ItjaGrandmother_flk & flk & $\begin{array}{l}\text { Bajdak/Tučkova 2004:59-60 } \\
\text { Morev et al 1981: 137-138 } \\
\text { Tučkova/Wagner-Nagy 2015: 122-123 }\end{array}$ & $\mathrm{Z}$ & Tym & 111 \\
\hline MNN_1977_VillageKuleevo_nar & nar & Morev et al 1981: 138 & $\mathrm{Z}$ & Tym & 74 \\
\hline MNS_1984_BrotherSister_flk & flk & Bykonia et al 1996: 109-119 & $\mathrm{Z}$ & Narym & 553 \\
\hline MNS_1984_DaughterOfEarth_flk & flk & Bykonia et al 1996: 99-106, 171-177 & $\mathrm{Z}$ & Narym & 306 \\
\hline MNS_NN_BullSon_flk & flk & Korobejnikova 2014: 25-28 & $\mathrm{Z}$ & Narym & 1146 \\
\hline PAV_NN_HowIBearCatch_nar & nar & Morev et al 1981: 131-137 & $\mathrm{Z}$ & Tym & 865 \\
\hline SAA_1971_MoleWife_flk & flk & Morev et al 1981: $122-123 \mid 124$ & $\mathrm{Z}$ & Narym & 256 \\
\hline SAA_1971_MoleWifēeVar_flk & flk & Morev et al 1981: $123 \mid 125$ & $\mathrm{Z}$ & Narym & 251 \\
\hline SAA_1971_ThreeSisters_flk & flk & Morev et al 1981: 125-131 & $\mathrm{Z}$ & Narym & 875 \\
\hline SAA_1984_MyGrandmother_nar & nar & Bykonia et al 1996: 95-96, 169-171 & $\mathrm{Z}$ & Narym & 115 \\
\hline SAG_1984_StoryAboutLife_nar & nar & Bajdak/Maksimova 2015: 133-149 & $\mathrm{Z}$ & Narym & 277 \\
\hline SAI_1984_StoryAboutLife_nar & nar & Bykonia et al 1996: 89-91, 167-169 & $\mathrm{Z}$ & Narym & 86 \\
\hline SAI_1984_StoryAboutLifeL̄ong_nar & nar & Bajdak/Maksimova 2015: 114-132 & $\mathrm{Z}$ & Narym & 365 \\
\hline SDP_1964_FairytaleBlackZar_flk & flk & Dul'zon1966b: 97-158 & $\mathrm{Z}$ & Narym & 7650 \\
\hline SVD_2002_StrongSelkup_flk & flk & Korobejnikova 2014: 11 & $\mathrm{Z}$ & Narym? & 52 \\
\hline TMN_1992_AffixedFoot_flk & flk & $\begin{array}{l}\text { Tučkova/Wagner-Nagy 2015: } 77 \\
\text { Tučkova 2002a:203 }\end{array}$ & $\mathrm{Z}$ & Narym & 23 \\
\hline TMN_1994_Cow_nar & nar & Korobejnikova 2014: 75 & $\mathrm{Z}$ & Narym & 98 \\
\hline TTD_1964_CalfHare_flk & flk & Szabó 1967: 24-28 & $\mathrm{Z}$ & Tym/Narym & 230 \\
\hline TTD_1964_CrowMagpie_flk & flk & Szabó 1967: 24 & $\mathrm{Z}$ & Tym/Narym & 36 \\
\hline TTD_1964_Frog_flk & flk & Szabó 1967: 34-46 & $\mathrm{Z}$ & Tym/Narym & 911 \\
\hline TTD_1964_IdzahKadzah_flk & flk & Szabó 1967: 22 & $\mathrm{Z}$ & Tym/Narym & 80 \\
\hline TTD_1964_PlaceWhereILive_nar & nar & Szabó 1967: 20 & $\mathrm{Z}$ & Tym/Narym & 42 \\
\hline TTD_1964_ReindeerDog_flk & flk & Szabó 1966: 254-255 & $\mathrm{Z}$ & Tym/Narym & 46 \\
\hline TTD_1964_Squirrel_nar & nar & Szabó 1967: 20 & $\mathrm{Z}$ & Tym/Narym & 15 \\
\hline TTD_1964_WildDucks_flk & flk & Szabó 1967: 28-32 & $\mathrm{Z}$ & Tym/Narym & 391 \\
\hline NN_1855_Hero1_song & song & Castrén1855(8): 340-344 & $\mathrm{Z} / \mathrm{S}$ & $\begin{array}{l}\text { Tym/Middle } \\
\text { Ob }\end{array}$ & 452 \\
\hline NN_1855_Hero2_song & song & Castrén1855(8): 345-356 & $\mathrm{Z} / \mathrm{S}$ & $\begin{array}{l}\text { Tym/Middle } \\
\text { Ob }\end{array}$ & 915 \\
\hline
\end{tabular}




\section{B Textsammlungen}

\begin{tabular}{lllll}
\hline Textname & Genre & Publikation & Dialekt Subdialekt & Tokens \\
\hline NN_1855_Hero3_song & song & Castrén1855(8): 356-364 & Z/S & $\begin{array}{l}\text { Tym/Middle } \\
\text { Ob }\end{array}$ \\
NN_1855_Hero4_song & song & Castrén1855(8): 365-369 & Z/S $\begin{array}{l}\text { Tym/Middle } \\
\text { Ob }\end{array}$ & 388 \\
\hline
\end{tabular}




\section{Abkürzungen und Symbole}

\begin{tabular}{|c|c|c|c|}
\hline 1 & 1. Person & INSTR & Instrumental \\
\hline 2 & 2. Person & INSTRN & Nomen instrumentalis \\
\hline 3 & 3. Person & INT.PF & Intensiv Perfektiv \\
\hline A & Agens & INTERJ & Interjektion \\
\hline $\mathrm{ABL}$ & Ablativ & INTERROG & Interrogativ \\
\hline ABST & Abstraktum & IPF & Imperfektiv \\
\hline ACC & Akkusativ & IPFV & Verbaler Imperfektivierer \\
\hline ACT & Nomen actionis & ITER & Iterativ \\
\hline $\mathrm{ADE}$ & Adessiv & LOC & Lokativ \\
\hline ADJ & Adjektiv & LOC.ADV & Adverbialer Lokativ \\
\hline ADJP & Adjektivphrase & $\mathrm{N}$ & Nomen \\
\hline ADJZ & Adjektivierer & NAR & Narrativer Text \\
\hline ADPP & Adpositionalphrase & NEG & Negationspartikel \\
\hline $\mathrm{ADP}$ & Adposition & NEG.EX & Negatives Existenzialverb \\
\hline ADV & Adverb & $\mathrm{NN}$ & Unbekannt \\
\hline ADVP & Adverbphrase & NOM & Nominativ \\
\hline ALL & Allativ & NP & Nominalphrase \\
\hline AN & Animat & NUM & Numeral \\
\hline AOR & Aorist & 0 & Objektive Konjugation \\
\hline CAP & Kaptativ & OBL & Oblique \\
\hline CAR & Karitiv & ONOM & Onomatopoeia \\
\hline CAUS & Kausativ & OPT & Optativ \\
\hline CLIT & Klitikon & ORD & Ordinal \\
\hline $\mathrm{COM}$ & Komitativ & $P$ & Patiens \\
\hline COND & Konditional & PART & Partikel \\
\hline CONJ & Konjunktion & PL & Plural \\
\hline CVB & Konverb & POM & Possessum \\
\hline DAT & Dativ & POS & Possessor \\
\hline DAT/ALL & Dativ/Allativ & $\mathrm{PP}$ & Postposition \\
\hline DEM & Demonstrativum & PROL & Prolativ \\
\hline DES & Desiderativ & PRON & Pronomen \\
\hline DETR & Detransivierer & PRS & $\operatorname{Pr} \square$ sens \\
\hline DIM & Diminutiv & PST & $\operatorname{Pr} \square$ teritum \\
\hline DRV & Derivationssuffix & PST.REP & Narratives $\operatorname{Pr} \square$ teritum \\
\hline DU & Dual & РTCP & Partizip \\
\hline DUR & Durativ & $\mathrm{PX}$ & Possessiv \\
\hline
\end{tabular}




$\begin{array}{llll}\text { EMPH } & \text { Emphatisches Pronomen } & \text { R } & \text { Rezipient } \\ \text { EP } & \text { Epenthetischer Vokal } & \text { REP } & \text { Reportativ } \\ \text { EX } & \text { Existential } & \text { RES } & \text { Resultativ } \\ \text { FLK } & \text { Folkloristischer Text } & \text { RFL } & \text { Reflexiv } \\ \text { FRQ } & \text { Frequentativ } & \text { S } & \text { Subjektive Konjugation } \\ \text { FUT } & \text { Futur } & \text { SG } & \text { Singular } \\ \text { GEN } & \text { Genitiv } & \text { SING } & \text { Singulativ } \\ \text { HAB } & \text { Habituativ } & \text { T } & \text { Thema } \\ \text { ILL } & \text { Illativ } & \text { TR } & \text { Transitiv } \\ \text { IMP } & \text { Imperativ } & \text { TRL } & \text { Translativ } \\ \text { INCH } & \text { Inchoativ } & \text { US } & \text { Usuativ } \\ \text { INDEF } & \text { Indefinit } & \text { V } & \text { Verb } \\ \text { INF } & \text { Infinitiv } & \text { VBLZ } & \text { Verbalisierer } \\ \text { INFER } & \text { Inferentialis } & \text { VP } & \text { Verbphrase }\end{array}$




\section{Literaturverzeichnis}

Alatalo, J. (1994): „De sölkupska dialekterna på övre Ob“. In: Journal de la Société finno-ougrienne, Jg. 85.

Alatalo, J./Donner, K./Sirelius, U. T. (2004): Sölkupisches Wörterbuch aus Aufzeichnungen von Kai Donner, U. T. Sirelius und Jarmo Alatalo. Bd. XXVII. Helsinki: Finnisch-Ugrische Ges.

Alitkina, L. A. (1985): „Otglagol'nye gruppy proizvodnyx imen prilagatel'nyx v sel'kupskom jazyke“. In: Jazyki i Toponimi, 5-9.

Anderson, G. D. S. (2004a): „Auxiliary Verb Constructions in Altai-Sayan Turkic“. In: Turcologica. Bd. 51: Harrassowitz: Wiesbaden,

- (2004b): „The languages of Central Sibiria: Introduction and overview“. In: Vajda, E. J. (Hg.): Languages and Prehistory of Central Siberia: Amsterdam [u.a.]: John Benjamin, 1-119.

Aydemir, İ. A. (2009): Konverbien im Tuwinischen. Eine Untersuchung unter Berücksichtigung des Altai-Dialekts. Bd. 80. Hrsg. von L. Johanson: Harrassowitz: Wiesbaden.

Bajdak, A. V./Tuchkova, N. A. (2004): Episody Ëposa ob It't'e"v chumil'kupskom dialektnom areale: Izdat. Tomskogo Gosudarstvennogo Architekturno-Stroitel'nogo Univ.

Bekker, Ė. G. (1995a): Morfologija sel'kupskogo jazyka : juzhnye dialekty Ch. 1. Tomsk: Tomskij Gosudarstvennyj Pedagogicheskij Institut.

- (1995b): Morfologija sel'kupskogo jazyka : juzhnye dialekty Ch. 2. Tomsk: Tomskij Gosudarstvennyj Pedagogicheskij Institut.

Bickel, B. (1991): Typologische Grundlagen der Satzverkettung. Ein Beitrag zur allgemeinen Grammatik der Satzverbindung und des Fährtenlegens.: Zürich: Seminar für Allgemeine Sprachwissenschaft der Universität.

- (1993): „Belhare subordination and the theory of topic“. In: Ebert, K. H. (Hg.): Studies in Clause Linkage. Papers from the First Köln-Zürich Workshop: Zürich: Seminar für Allgemeine Sprachwissenschaft der Universität, 23-55.

- (2010): „Capturing particulars and universals in clause linkage: a multivariate analysis". In: Brill, I. (Hg.): Clause Linking and Clause Hierarchy: Syntax and Pragmatics: John Benjamins: Amsterdam, 51-102.

Bradley, J. (2016): Mari Converb Constructions: Productivity and Regional Variance. Unveröffentlichte Dissertation. Universität Wien.

Budzisch, J. (2015): „Possession in Southern Selkup“. In: Tomsk Journal of Linguistics and Anthropology, Jg. 4, 45-50.

- (2017a): „Locative, existential and possessive sentences in Selkup dialects“. In: Finnisch-Ugrische Mitteilungen, Jg. 41, 45-62. 
Budzisch, J. (2017b): „On the non-possessive use of possessive suffixes in Central and Southern Selkup“. In: Ural-Altaic Studies, Jg. 25, 58-66.

Budzisch, J./Harder, A./Wagner-Nagy, B. (2019): Selkup Language Corpus (SLC). Archived in Hamburger Zentrum für Sprachkoropra. Version 1.0.0. Publication date 2019-02-08. - Zugriff am 20. November 2019.

Bykonja, V. V. (2005): Sel'kupsko-russkij dialektnyj slovar'. Tomsk: Izdat. Tomskogo Gosudarstvennogo Pedagogičeskogo Univ.

Castrén, M. A. (1854): Grammatik der samojedischen Sprachen. Bd. 7. Hrsg. von A. Schiefner. St. Petersburg: Kaiserliche Akad. der Wiss.

- (1855): Wörterverzeichnis aus den samojedischen Sprachen. Bd. 8. Hrsg. von A. Schiefner. St. Petersburg: Kaiserliche Akad. der Wiss.

Castrén, M. A./Lehtisalo, T. V. (1940): Samojedische Sprachmaterialien. Helsinki: Suomalais-Ugrilainen Seura.

Cheremisina, M. I./Martynova, E. (1991): Sel'kupskij glagol. Formy i ix sintaksicheskie funkcii. Novosibirsk: Novosibirskij gosudarstvennyj un-t.

Comrie, B. (2008): „Subordination, Coordination. Form, Semantics, Pragmatics Setting the Scene“. In: Vajda, E. J. (Hg.): Subordination and Coordination Strategies in North Asian Languages: John Benjamins: Amsterdam, 1-16.

Comrie, B. (1988): „General features of the Uralic languages“. In: Sinor, D. (Hg.): The Uralic Languages. Description, History and Foreign Influences. Bd. 1: Niederlande: Brill, 451-477.

Décsy, G. (1966): Yurak Chrestomathy. Bd. 50: Indiana: Indiana University Press.

Dixon, R. M. W. (1997): The rise and fall of languages: Cambridge University Press.

- (2010a): Basic linguistic theory. Bd. 1 Methodology: Oxford [u.a.]: Oxford Univ. PressOxford Univ. Press.

- (2010b): Basic linguistic theory. Bd. 2 Grammatical topics: Oxford [u.a.]: Oxford Univ. Press.

- (2012): Basic linguistic theory. Bd. 3 Further grammatical topics: Oxford [u.a.]: Oxford Univ. Press.

Dryer, M.S. (2006a): „Descriptive theories, explanatory theories, and basic liguistic theory“. In: Catching language: the standing challenge of grammar writing: Berlin [u.a.]: de Gruyter, 207-234.

- (2006b): „Functionalism and the theory-metalanguage confusion“. In: Phonology, morphology, and the empirical imperative: Papers in honour of Bruce Derwing: Taipei: The Crane Publishing Company, 27-59.

- (2007): „Noun phrase structure“. In: Shopen, T. (Hg.): Bd. 2: Cambridge: Cambridge University Press, 151-205.

Dul'zon, A. P. (1966a): Ketskie skazki: Tomsk: Izd. Univ.

- (1966b): „Sel'kupskie skazki“. In: Donner, K. (Hg.): Jazyki i toponimija Sibiri.

Bd. 1: Tomsk: Izd. Tomskogo Univ., 96-158.

Dul'zon, A. P./Porotova, T. I. (1976): Skazki narodov Sibirskogo Severa [Teksty i per.] Bd. 2: Tomsk: Izd. Tomskogo Univ.

- (1980): Skazki narodov Sibirskogo Severa [Teksty i per.] Bd. 3: Tomsk: Izd. Tomskogo Univ. 
Evans, N./Dench, A. (2006): „Introduction: Catching language“. In: Catching language: the standing challenge of grammar writing: Berlin [u.a.]: Mouton de Gruyter, 1-39.

Fil'chenko, A. J. (2010): Annotirovannye fol'klornye teksty Obsko-Enisejskogo jazykovogo areala: Tomsk: Veter.

Fokos-Fuchs, D. (1962): Rolle der Syntax in der Frage nach Sprachverwandtschaft : mit besonderer Rücksicht auf das Problem der ural-altaischen Sprachverwandtschaft. Bd. 11: Wiesbaden: Harrassowitz.

Foley, W./Van Valin Jr., R. (1984): Functional syntax and universal grammar: Cambridge: Cambridge University Press.

Freeze, R. (1992): „Existentials and other locatives“. In: Language, Jg. 68, 553-595.

Gippert, J./Himmelmann, N. P./Mosel, U. (2006): Essentials of language documentation: Berlin [u.a.]: Mouton de Gruyter.

Glushkov, S. V./Bajdak, A. V. (2016): „O dialektnoj prinadlezhnosti chetyrech geroicheskix pesen M. A. Kastrena“. In: Linguistica Uralica, Jg. 4.

Glushkov, S. V./Bajdak, A. V./Maksimova, N. P. (2011): „Dialekty sel'kupskogo jazyka“. In: Tuchkova, N. A. et al. (Hgg.): Sel'kupy. Ocherki tradicionnoj kul'tury sel'kupskogo jazyka. Tomsk.

Grenoble, L. A. (2014): „Spatial semantics, case and relator nouns in Evenki“. In: Suihkonen, P./Whaley, L. J. (Hgg.): On diversity and complexity of languages spoken in Europe and North and Central Asia. Bd. XVIII. Amsterdam: John Benjamins Publishing Company, 111-131.

Grigorovskij, N.P. (1879): Azbuka sjussogoj gulani: dlja innostrancev' Narymskogo kraja. Kazan'.

Haiman, J./Thompson, S. (1984): „Subordination in universal grammar“. In: Brugmann, C./Macauley, M. (Hgg.): Proceedings of the tenth annual meeting of the Berkeley linguistics society: Berkeley, CA: University of California Press, 510-523.

Hajdú, P. (1968): Chrestomathia Samoiedica. Budapest: Tankönyvkiadó, 239 S.

- (1973): Die erste sölkupische Fibel. Aus dem Jahre 1879. Bd. 2. Photomechan. Nachdr. Szeged: Univ. Szegediensis de Attila József Nominata.

- (1975): Samojedologische Schriften. Bd. 6. Szeged: Univ. Szegediensis de Attila József Nominata.

Harder, A. (2017): „Grammaticalization of spatial expressions in Central and Southern Selkup“. In: Finnisch-Ugrische Mitteilungen, Jg. 41, 153-174.

Haspelmath, M. (1995): „The converb as a cross-linguistically valid category“. In: Haspelmath, M./König, E. (Hgg.): Bd. 13. Converbs in Cross-Linguistic Perspective: Berlin [u.a.]: Mouton de Gruyter, 1-55.

- (1999): „Converb“. In: Brown, K./Miller, J. (Hgg.): Concise encyclopedia of grammatical categories: Amsterdam: Elsevier, 110-115.

Heine, B. (2009): Possession: cognitive sources, forces and grammaticalization. Cambridge u.a.: Cambridge University Press.

Helimski, E. (1983): The language of the first Selkup books. Szeged: Univ. Szegediensis de Attila József Nominata. 
Helimski, E. (1998): „Selkup“. In: Abondolo, D. M. (Hg.): The Uralic languages. London u.a.: Routledge, 480-515.

Himmelmann, N. P. (1998): „Documentary and Descriptive Linguistics“. In: Linguistics, Jg. 36, 161-195.

Janhunen, J. (1977): Samojedischer Wortschatz: gemeinsamojedische Etymologien.

- (1998): „Samoyedic“. In: Abondolo, D. (Hg.): The Uralic Languages. London u.a.: Routledge, 457-479.

- (2002): „The Nenets Imperative Sentence and its Background.“ In: FinnischUgrische Mitteilungen Nr. 24/25, 71-85.

Johanson, L. (1971): Aspekt im Türkischen. Uppsala.

- (1995): „On Turkic converb clauses“. In: Haspelmath, M./König, E. (Hgg.): Berlin [u.a.]: Mouton de Gruyter, 313-347.

- (2000): „Turkic indirectives“. In: Bo, L./Bo, U. (Hgg.): Berlin [u.a.]: Mouton de Gruyter, 61-87.

Kahr, J. C. (1975): „Adpositions and locationals: Typology and diachronic development“. In: Working papers on language universals, Jg. 19, 21-54.

Katz, H. (1975): Selcupica I. Materialien von Tym. München: Finnisch-Ugrisches Seminar der Universität.

- (1988): Die Märchen in Grigorovskis Azbuka. Transkription - Übersetzung - Kommentar: München: Finnisch-Ugrisches Seminar der Univ.

Katzschmann, M. (2008): Chrestomathia Nganasanica: Norderstedt: Books on Demand.

Keenan, E. L./Comrie, B. (1977): „Noun Phrase Accessibility and Universal Grammar“. In: Linguistic Inquiry, Jg. 8(1), 63-99.

Klumpp, G. (2002): Konverbkonstruktionen im Kamassischen. Wiesbaden: Harrassowitz: Wiesbaden: Harrassowitz.

- (2005a): „Aspect markers grammaticalized from verbs in Kamas“. In: Acta Linguistica Hungarica, Jg. 52 (4), 397-409.

- (2005b): „A List of Kamas Tense-Aspect-Forms“. In: Wagner-Nagy, B. (Hg.): Mikola-konferencia 2004: Szeged, 41-61.

Kovylin, S. V. (2015): „Об отрицании при местоимениях и наречиях в центральных диалектах селькупского языка“. In: Вестник Томского государственного университета, Jg. 4, Nr. 157, 36-42. Kovylin. 〈https : // cyberleninka . ru / article / n / ob-otritsanii - pri - mestoimeniyah - i narechiyah-v-tsentralnyh-dialektah-selkupskogo-yazyka/viewer $\rangle$.

Kovylin, S. (2015): Отрицание и способы его выражения в ваховском и васюганском диалекте хантыйского языка, центральных и язных диалектах селькупского языка. [Negation and its expression in the Vakh and Vasyugan dialect of Khanty and in Central and Southern dialects of Selkup.] Unveröffentlichte Dissertation. Tomsk.

- (2016): „Negative Pronouns and Adverbs in the Central and Southern Dialects of Selkup“. In: Finnisch-Ugrische Mitteilungen, Jg. 40, 47-72.

Kuz'mina, A. I. (1967): „Dialektologicheskie materialy po sel'kupskomu jazyku“. In: Issledovanija po jazyku i fol'kloru, Jg. 2, 267-329. 
- (1974): Grammatika sel'kupskogo jazyka. 1: Novosibirsk: Novosibirskij gosudarstvennyj univ.

Kuzmina, A. J. (1974): „A szelkup fonémarendszer kérdéséhez“. In: Nyelvtudományi Közlemények, Jg. 76, 135-156.

Kuznecova, A. I./Chelimskij, E./Grushkina, E. V. (1980): Ocherki po sel'kupskomu jazyku tazovskij dialekt. 1: Moskva: Izdatel'stvo Moskovskogo Universiteta.

Kuznecova, N. G. (1995): Grammaticheskie kategorii juzhnosel'kupskogo glagola: Tomsk: Izd. Tomskogo Universiteta.

Laakso, J. (2011): „The Uralic languages“. In: Kortmann, B./Auwera, J. v. d. (Hgg.): The Languages and Linguistics of Europe. A Comprehensive Guide. Bd. 1: Berlin [u.a.]: Mouton de Gruyter, Kap. 8. 179-197.

Lehmann, C. (1988): „Towards a Typology of Clause Linkage“. In: Haiman, J./ Thompson, S. (Hgg.): Clause Combining in Grammar and Discourse: Amsterdam: Benjamins, 181-225.

Lemskaya, V. (2012): „A Chulym Turkic Text. Handsome-brown“. In: Fil'chenko, A. J. (Hg.): Annotated Folk Texts of Ob-Yenisei Area. Bd. V-2 Pp: Tomsk: Vajar, 184-237.

Longacre, R. E. (2007): „Sentences as combinations of clauses“. In: Shopen, T. (Hg.): Language Typology and Syntactic Description. Bd. II: Cambridge University Press: Cambridge [et al.], Kap. 7. 372-420.

Lyons, J. (1969): „A note on possessive, existential and locative sentences“. In: Foundation of Language, Jg. 3, 390-396.

Malchukov, A./Haspelmath, M./Comrie, B. (2010): Studies in ditransitive constructions. A comparative handbook. Berlin: Berlin [u.a.]: Mouton de Gruyter.

Malchukov, A./Ogawa, A. (2011a): Impersonal constructions: a cross-linguistic perspective. Hrsg. von A. Malchukov und A. Siewierska. Amsterdam/ Philadelphia: John Benjamins.

- (2011b): „Meteorological verbs in Uralic languages - are there any impersonal structures to be found *“. In: Malchukov, A./Siewierska, A. (Hgg.): Impersonal constructions: a cross-linguistic perspective. Amsterdam/ Philadelphia: John Benjamins, 395-438.

Miestamo, M. (2005a): Standard Negation. The Negation of Declarative Verbal Main Clauses in a Typological Perspective: Berlin [u.a.]: Mouton de Gruyter.

- (2005b): „Symmetric and asymmetric standard negation“. In: Dryer, M. S. et al. (Hgg.): World Atlas of Language Structures: Oxford, 458-461.

Mikola, T. (2004): Studien zur Geschichte der samojedischen Sprachen. Hrsg. von B. Wagner-Nagy: Szeged: SzTE Finnisch-Ugrisches Institut.

Nedjalkov, V. (1995): „Some typological parameters of converbs“. In: Haspelmath, M./König, E. (Hgg.): Converbs in Cross-Linguistic Perspective. Bd. 13. Converbs in Cross-Linguistic Perspective: Berlin [u.a.]: Mouton de Gruyter, 97-136.

Nikolaeva, I. (2014): A Grammar of Tundra Nenets. Bd. 65: Berlin [u.a.]: Mouton de Gruyter.

Payne, T. E. (1995): Describing morphosyntax. A guide for field linguistics: Cambridge University Press. 
Pischlöger, C. „Hilfsverbverbindungen in einigen uralischen und nichturalischen Sprachen“. In: Congressus Nonus Internationalis Fenno-Ugristarum 7.-13.8. 2000. Hrsg. von T. Seilenthal. Bd. Pars IV: Dissertationes sectionum: linguistica 1. Tartu: Eesti Fennougristide Komitee [u.a.], 45-52.

Prokof'ev, G. N. (1931): „Materialien zur Erforschung der ostjak-samojedischen Sprache. Die Tasovsche Mundart“. In: Ungarische Jahrbücher 2, 91-98, 293-297, 440-452.

- (1935): Sel'kupskij (Ostjako-Samojedskij) Jazyk: Volume 1. Sel'kupskaja Grammati$k a$ : Leningrad: Uchpedgiz.

- (1937): „Sel'kupskij (ostjak-samoedskij) jazyk“. In: Jazyki i pis’mennost' narodov Severa. Bd. 1: Leningrad: Uchpedgiz, 91-124.

Prokof'ev, G. N./Prokof'eva, E. D. (1940): Bukvar'. Leningrad: Uchpedgiz.

Prokof'eva, E. D. (1932): Narqb wattb posukol sel qumbl togbltbhsatbl nəkbr: Leningrad: Uchpedgiz.

- (1953): Bukvar': Leningrad: Uchpedgiz.

Ramstedt, G. J. (1903): Über die Konjugation des Khalka-Mongolischen. Bd. 19: Helsinki: Société Finno-Ougrienne.

Rice, K. (2006a): „Let the language tell its story?“ In: Catching language: the standing challenge of grammar writing: Berlin [u.a.]: de Gruyter, 235-268.

- (2006b): „A typology of good grammars“. In: Studies in Language, Jg. 30, Nr. 2, 385-415.

Sasse, H.-J. (1991): „Aspekttheorie“. In: Arbeitspapiere des Institus für Sprachwissenschaft der Universität zu Köln. Bd. 14 (neue Folge): Köln: Universität Köln, 1-35.

Schmidt, T./Wörner, K. (2005): „Erstellen und Analysieren von Gesprächskorpora mit EXMARaLDA“. In: Gesprächsforschung: Online-Zeitschrift zur verbalen Interaktion. Bd. 6: Mannheim: Verlag für Gesprächsforschung, 171-195.

Schönig, C. (1984): Hilfverben im Tatarischen. Untersuchungen zur Funktionsweise einiger Hilfsverbverbindungen: Wiesbaden: Steiner.

Siegl, F. (2013): Materials on Forest Enets, an indigenous language of Northern Siberia: Helsinki: Société Finno-Ougrienne.

Siewierska, A. (2008): „Introduction: Impersonalization: An agent-based vs. a subject-based perspective." In: Transactions of the Philological Society (Special issue on Impersonal Constructions in Grammatical Theory, guest-edited by A. Siewierska), Jg. 106 (2), 115-137.

Sinnemäki, K. (2014): „A typological perspective on Differential Object Marking“. In: Linguistics, Jg. 52, Nr. 2, 281-313.

Sobanski, F. (2001): „The Southern Selkups of Tomsk province before and after 1991“. In: Nationalities Papers: The Journal of Nationalism and Ethnicity, Jg. 29, Nr. 1, 171-179.

Sokolova, Z. P. (1970): „Socialnaja organizacija obskih ugrov i sel'kupov“. In: $O b$ shchestvennoj stroj u narodov Severnoj Sibiri (XVII - nachalo XX. vekov): Leningrad: Nauka, 103-153. 
Stassen, L. (2001): „Predicative Possession“. In: Haspelmath, M./Oesterreicher, W./Raible, W. (Hgg.): Language Typology and Languages Universals. Bd. 2: Berlin [u.a.]: Mouton de Gruyter, 954-960.

Tereshchenko, N. M. (1973): Sintaksis samodijskich jazykov. Prostoe predlozhenie: Leningrad: Nauka.

- (1979): Nganasanskij jazyk: Leningrad: Nauka.

Thompson, S./Longacre, R. E./Hwang, S. J. J. (2007): „Adverbial clauses“. In: Shopen, T. (Hg.): Language Typology and Syntactic Description. Bd. II. Complex Constructions: Cambridge: Cambridge University Press, 237-300.

Tikkanen, B. (2001): „Converbs“. In: Haspelmath, M. (Hg.): Bd. 20, Halbb. 2: Berlin [u.a.]: Mouton de Gruyter, 1112-1123.

Tuchkova, N. A./Kuznetsova, N. G. (2010): „Die Südselkupen und die südlichen Grenze ihres Verbreitungsgebietes“. In: Finnisch-Ugrische Mitteilungen, Jg. 32/33, 595-605.

Tuchkova, N. A./Helimski, E. (2010): O materialah A. I. Kuz'minoj po sel'kupskomu jazyku [= Über die selkupischen Sprachmaterialien von Angelina I. Kuz'mina]: Hamburg: Universität Hamburg.

Valijärvi, R.-L. (2008): „Converbs in Northern Selkup“. In: Subordination and Coordination Strategies in North Asian Languages: Amsterdam [u.a.]: John Benjamins, 167-177.

Van Valin Jr., R. (2005): Exploring the syntax-semantics interface: Cambridge: Cambridge University Press.

Voevodina, N. M. (1976): „Funkcii deeprichastij i deeprichastnyx oborotov v predlozhenii selk'kupskogo jazyka“. In: Jazyki i Toponimi, Jg. 1, 92-102.

Wagner-Nagy, B. (2011): On the typology of negation in Ob-Ugric and Samoyedic languages. Bd. 262. Helsinki: Soc. Finno-Ougrienne.

- (2016): „Existentials, Possessives and Definiteness in Samoyedic Languages“. In: Fischer, S./Kupisch, T./Rinke, E. (Hgg.): Definiteness Effects: Bilingual, Typological and Diachronic Variation: Cambridge: Cambridge Scholars Publishing, 213-243.

- (2019): A Grammar of Nganasan. Hrsg. von B. Wagner-Nagy und V. Gusev: Brill: Leiden, Boston.

Wagner-Nagy, B. (2015): „Negation in Selkup“. In: Miestamo, M./Tamm, A./ Wagner-Nagy, B. (Hgg.): Negation in Uralic languages: Amsterdam [u.a.]: John Benjamin,

Wagner-Nagy, B./Szeverényi, S. (2013): „On the argument structure of the 'give' verbs in Nganasan and in Selkup“. In: Tomsk journal of linguistics and anthropology Nr. 1, 27-37.

Wegener, H. (2015): Differentielle Objektmarkierung im Selkupischen (unpublished). Magisterarb. Universität Hamburg.

- (2018): „On differential object marking in Southern and Central Selkup“. In: Journal of Estonian and Finno-Ugric Linguistics, Jg. 9, 169-186.

Wickman, B. (1955): The Form of the object in the Uralic languages: Uppsala: Almqvist und Wiksell.

Winkler, E. (2011): Udmurtische Grammatik. Bd. 81: Wiesbaden: Harrassowitz. 
Woodbury, T. (2003): „Defining documentary linguistics“. In: Language Documentation and Description. Bd. 1: London : SOAS, Univ. of London, 35-51.

Wörner, K. (2010): Werkzeuge zur flachen Annotation von Transkriptionen gesprochener Sprache (Dissertation): Hamburg: Universität Hamburg. 


\section{Stichwortverzeichnis}

Adjektivphrase, siehe Phrase Adpositionalphrase, siehe Phrase Adverbialsatz, siehe Komplexer Satz Adverbphrase, siehe Phrase

Benefaktiv, 109

Differentielle Objektmarkierung, 17, 92-95, 101-102, 210

DOM, siehe Differentielle Ojektmarkierung

Einfache Postpositionen, $62-67$

Einfacher Satz, $99-146$

Deklarativsatz, 99-116, 133-135, 211

Ditransitiver Satz, $102,110,135$

Existenzialsatz, 115-116, 119, 137-139, 211

Imperativsatz, 131-133, 142-144

Interrogativsatz, 126-131, 135137, $144-145$

Kopulasatz, 110-116, 137-142,

Lokativsatz, 114 115, 118-119, $139-140,211$

Possessivsatz, 116, 119-120, 140142,211

Verbloser Satz, 116-120, 142

Existenzialsatz, siehe Einfacher Satz, 133

Grammatische Relationen, $89-97$

Imperativsatz, siehe Einfacher Satz Infinite Verbformen, siehe Komplexer Satz

Interrogativsatz, siehe Einfacher Satz
Juxtaposition, siehe Komplexer Satz

Komparation, 60, 64, 112, 113

Komplementsatz, siehe Komplexer Satz

Komplexe Postpositionen, 68-75

Komplexer Satz, 147-182, 185, 211

Adverbialsatz 149, 157-158, 162 $168,177-181$

Infinite Verbformen, 149-151.

$157-159,174-175,211$

Juxtaposition, 148, 155, 171-172

Komplementsatz, 160-162, 175177

Konjunktionalsatz, 151-155, 159168,173

Koordination, $147-155,170-173$, 212

Subordination, 147, 155-168, 170. 174-181, 212

Konjunktionalsatz, siehe Komplexer Satz

Konverbale Prädikate, $189-207$

Konverben, 149-151, 157-158, 173174, 185-207, 211-212

Koordination, siehe Komplexer Satz

Kopulasatz, siehe Einfacher Satz

Lokativsatz, siehe Einfacher Satz

Modifikatoren

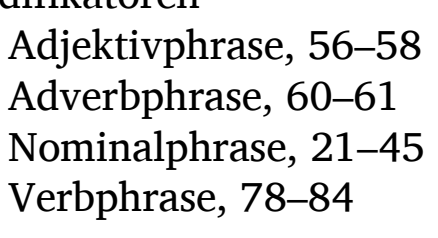

Negation, 17, 45-54, 59, 61, 84-87, 


\section{$133-145,170-182,210,212$ 213}

Nominalphrase, siehe Phrase

Nonverbale Prädikate, siehe Kopulasatz und Verblose Sätze

Objektive Konjugation, 17, 210

Phrase, 19, 87, 209-210

Adjektivphrase, 56-59

Adpositionalphrase, $62-75$

Adverbphrase, 60-61

Nominalphrase, $19-54$

Verbphrase, 76-88, 210

Possessivität, 16, 22, 27, 110, 114, 116, $118-119,211$

Possessivsatz, siehe Einfacher Satz

Postposition, siehe Adpositionalphrase
Präpositionen, 7374

Relationale Nomen, siehe Komplexe Postpositionen

Relativsatz, 168-169, 181, 209

Satzglieder, $89-97$

Subjektive Konjugation, 17, 210

Subordination, siehe Komplexer Satz

Typologisches Profil, 15-18

Unpersönlicher Satz, $120-125$

Verblose Sätze, siehe Einfacher Satz Verbphrase, siehe Phrase

Wortstellung, 97-98, 210-211 



\section{[n'ejaqulamdat matčewl'e wett'aqusadat]}

Das Selkupische ist eine akut vom Aussterben bedrohte samojedische Sprache. Dessen zentrale und südliche Dialekte sind Forschungsgegenstand der vorliegenden Arbeit. Sie ist eine formbasierte, vorrangig synchrone Beschreibung der grundlegenden syntaktischen Strukturen (Phrasenstrukturen, einfache und komplexe Sätze) und basiert auf einem Korpus gesprochener Sprache, dessen publizierte Texte im Verlauf von 130 Jahren gesammelt wurden.

\section{[na quila surrum qwatlewl'e]}

\section{Logos Verlag Berlin}

ISBN 978-3-8325-5182-7 